\title{
Ruthenium-Catalyzed Brook Rearrangement Involved Domino Sequence Enabled by Acylsilane-Aldehyde Corporation
}

Xiunan Lu, Jian Zhang, ${ }^{*}$ Liangyao Xu, Wenzhou Shen, Feifei Yu, Liyuan Ding, and Guofu Zhong*

College of Materials, Chemistry and Chemical Engineering, Hangzhou Normal University, Hangzhou 311121,

\section{China}

zhangjian@hznu.edu.cn; zgf@hznu.edu.cn

\section{Supporting Information}

\section{Table of Contents}

General Methods $\quad$ SI-2

General Procedure for the Synthesis of Aroylsilanes $\quad$ SI-3

$\begin{array}{lr}\text { Characterization Data of Aroylsilanes } & \text { SI-5 }\end{array}$

General Procedure for the Synthesis of Silyloxy Indenes $\quad$ SI-13

$\begin{array}{lr}\text { Characterization Data of Silyloxy Indenes } & \text { SI-14 }\end{array}$

$\begin{array}{lr}\text { Controlled Experiments } & \text { SI-29 }\end{array}$

$\begin{array}{lr}\text { Deuterium Labeled Experiments } & \text { SI-35 }\end{array}$

$\begin{array}{ll}\text { Application Development } & \text { SI-40 }\end{array}$

$\begin{array}{lr}\text { References } & \text { SI-44 }\end{array}$

${ }^{1} \mathrm{H},{ }^{13} \mathrm{C}$ NMR Spectra $\quad$ SI-45 


\section{General Methods}

Analytical thin layer chromatography (TLC) was performed using Merck 60 F254 precoated silica gel plate ( $0.2 \mathrm{~mm}$ thickness). Subsequent to elution, plates were visualized using UV radiation (254 nm) on Spectroline Model ENF-24061/F $254 \mathrm{~nm}$. Further visualization was possible by staining with basic solution of potassium permanganate or acidic solution of ceric molybdate. Flash column chromatography was performed using Merck aluminium oxide 90 active neutral with freshly distilled solvents. Columns were typically packed as slurry and equilibrated with the appropriate solvent system prior to use. Proton nuclear magnetic resonance spectra ( ${ }^{1} \mathrm{H}$ NMR) were recorded on Bruker AMX 500 spectrophotometer $\left(\mathrm{CDCl}_{3}\right.$ as solvent). Chemical shifts for ${ }^{1} \mathrm{H}$ NMR spectra are reported as $\delta$ in units of parts per million (ppm) downfield from $\mathrm{SiMe}_{4}(\delta 0.0)$ and relative to the signal of chloroform-d ( $\delta 7.26$, singlet). Multiplicities were given as: s (singlet), d (doublet), $\mathrm{t}$ (triplet), dd (doublets of doublet) or m (multiplets). The number of protons ( $\mathrm{n}$ ) for a given resonance is indicated by $\mathrm{nH}$. Coupling constants are reported as a $J$ value in $\mathrm{Hz}$. Carbon nuclear magnetic resonance spectra $\left({ }^{13} \mathrm{C}\right.$ NMR) are reported as $\delta$ in units of parts per million (ppm) downfield from $\mathrm{SiMe}_{4}(\delta 0.0)$ and relative to the signal of chloroform-d ( $\delta 77.0$, triplet). Mass spectrometry was performed by Waters Q-Tof Premier Micromass instrument, using Electro Spray Ionization (ESI) mode. IR spectra were recorded as thin films on KBr plates on a Bio-Rad FTS165 FTIR spectrometer and are reported in frequency of absorption $\left(\mathrm{cm}^{-1}\right)$. $\left[\mathrm{Ru}(p \text {-cymene }) \mathrm{Cl}_{2}\right]_{2}, \mathrm{AgSbF}_{6}$ and $\mathrm{Cu}(\mathrm{OAc})_{2}$ were purchased from TCI and used directly. Other reagents, unless otherwise noted below, are commercially available from TCI, Energy Chemical, Alfa Aesar (China) Chemical Co. Ltd. and used without further purification. Aroylsilanes were prepared from aroyl chlorides by reported method (1a-1t) ${ }^{[1]}$ 


\section{General Procedures for the Synthesis of Aroylsilanes}

\section{General procedure 1: synthesis of aroylsilanes 1a-1 $\mathrm{t}^{[1]}$}

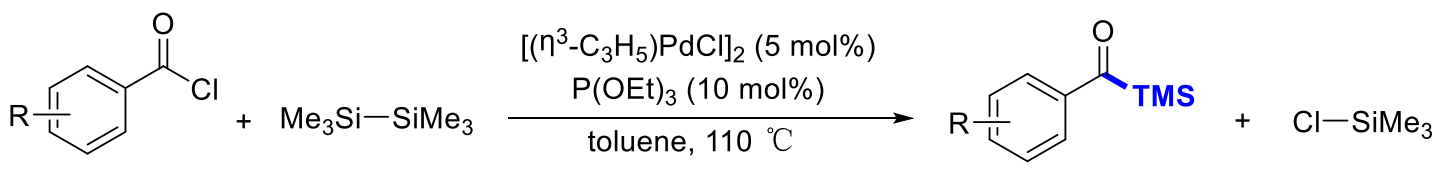

Aroylsilanes 1a-1t were prepared according to reported literature. ${ }^{[1]}$ Acid chloride (1.0 eq., 5.0 mmol) was added in dropwise to a stirred solution of allylpalladium chloride dimer ( $5 \mathrm{~mol} \%, 0.25$ mmol, $91.5 \mathrm{mg})$, triethyl phosphite $(10 \mathrm{~mol} \%, 0.5 \mathrm{mmol}, 83.1 \mathrm{mg})$ and hexamethyldisilane $(1.65$ eq., $8.25 \mathrm{mmol}, 1.2 \mathrm{~g})$ in toluene $(3 \mathrm{~mL})$ at $0{ }^{\circ} \mathrm{C}$ and stirred for 5 minutes. This mixture was stirred at $110^{\circ} \mathrm{C}$ in an oil bath for overnight. After cooling to room temperature, the reaction was transferred to eggplant flask and concentrated under reduced pressure. The residue was separated by flash chromatography on silica gel $(\mathrm{PE}: \mathrm{EA}=600: 1)$ to afford the aroylsilanes.

\section{General procedure 2: synthesis of aroylsilanes $1 \mathrm{u}$ and $1 \mathrm{v}^{[2]}$}

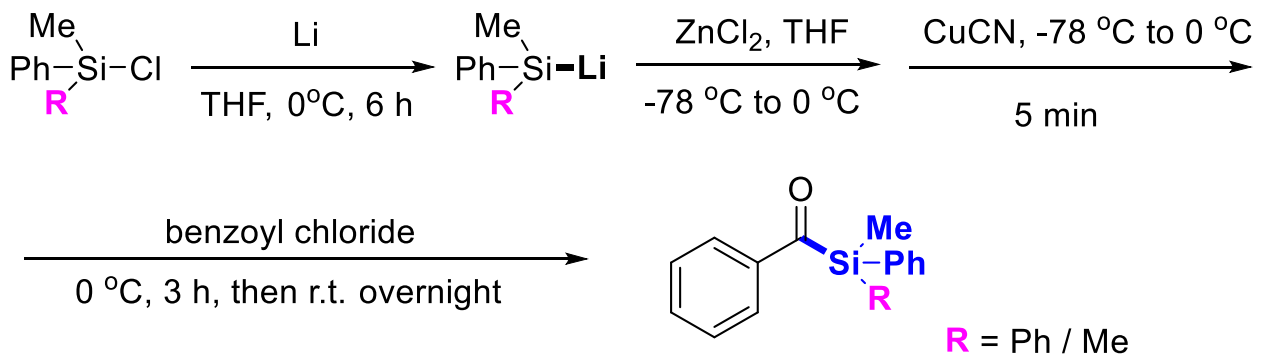

Chlorosilane $(0.5 \mathrm{~mL}, 3 \mathrm{mmol})$ was added to a suspension of $\mathrm{Li}(0.208 \mathrm{~g}, 30 \mathrm{mmol})$ in THF $(2.5$ $\mathrm{mL}$ ) at $0{ }^{\circ} \mathrm{C}$ and stirred for $6 \mathrm{~h}$, and a deep red solution was obtained. The obtained solution of silyllithium in THF $(4.5 \mathrm{~mL})$ was treated with a $1 \mathrm{M}$ solution of $\mathrm{ZnCl}_{2}$ in $\mathrm{Et}_{2} \mathrm{O}(3 \mathrm{~mL}, 3 \mathrm{mmol})$ at $78^{\circ} \mathrm{C}$ and then raised to $0{ }^{\circ} \mathrm{C}$ under $\mathrm{Ar}$, while the colour of the solution changed from dark to yellow. The resulting solution was transferred via cannula to a suspension of $\mathrm{CuCN}(0.134 \mathrm{~g}, 1.5 \mathrm{mmol})$ in THF (6 mL), stirred at $-78{ }^{\circ} \mathrm{C}$ and allowed to warm to $0{ }^{\circ} \mathrm{C}$, and a dark violet solution was obtained. The benzoyl chloride $(0.211 \mathrm{~g}, 1.5 \mathrm{mmol})$ was then added via syringe to the silyi-zinc cuprate at $0{ }^{\circ} \mathrm{C}$. After stirring for $3 \mathrm{~h}$ at $0{ }^{\circ} \mathrm{C}$ and overnight at r.t., the reaction was quenched by the addition of 
Sat. aq $\mathrm{NH}_{4} \mathrm{Cl}$ and extracted with EtOAc. The organic layer was dried over anhydrous $\mathrm{Na}_{2} \mathrm{SO}_{4}$ and then concentrated under reduced pressure. The products were purified by flash chromatography on silica gel $(\mathrm{PE}: \mathrm{EA}=800: 1)(\mathbf{2 v}, \mathrm{R}=\mathrm{Ph}, 226.6 \mathrm{mg}$, yield $=50 \%$; 2u, $\mathrm{R}=\mathrm{Me}, 151.3 \mathrm{mg}$, yield $=$ $42 \%)$.

\section{General procedure 3: synthesis of aroylsilane $1 w^{[3]}$}

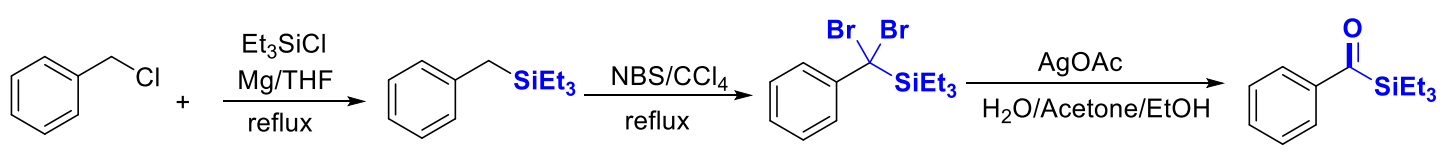

Step 1: preparation of benzylsilane

Activated $\mathrm{Mg}$ powder (1.1 equiv, $5.5 \mathrm{mmol}, 133.7 \mathrm{mg}$ ) was added to THF $(6.25 \mathrm{~mL})$ at $0{ }^{\circ} \mathrm{C}$, and then the benzyl chloride (1.0 equiv, $5.0 \mathrm{mmol}, 633 \mathrm{mg}$ ) was added. After stirring for $1 \mathrm{~h}$ at $0{ }^{\circ} \mathrm{C}$, then the mixture was heated to reflux in an oil bath and the chlorotriethylsilane (1.4 equiv, $7.0 \mathrm{mmol}$, $1.055 \mathrm{~g}$ ) was added in dropwise. After $12 \mathrm{~h}$, the mixture was cooled to $0{ }^{\circ} \mathrm{C}$ and the reaction was quenched with saturated aqueous $\mathrm{NH}_{4} \mathrm{Cl}$. The mixture was allowed to warm to ambient temperature, then taken up in EtOAc and washed three times with EtOAc. The combined organic layer was dried over anhydrous $\mathrm{Na}_{2} \mathrm{SO}_{4}$, filtered and concentrated in vacuo. The arylsilanes products were purified by flash chromatography on silica gel $(\mathrm{PE})(927.7 \mathrm{mg}$, yield $=90 \%)$.

Step 2: preparation of $\alpha, \alpha$-dibromo benzylsilane

Arylsilane (1.0 equiv, $4.5 \mathrm{mmol}, 927.7 \mathrm{mg})$, NBS (2.1 equiv, $9.45 \mathrm{mmol}, 1.68 \mathrm{~g})$ and AIBN (0.05 equiv, $0.225 \mathrm{mmol}, 36.9 \mathrm{mg})$ were added to $\mathrm{CCl}_{4}(22.5 \mathrm{~mL})$. The mixture was heated to reflux in an oil bath and maintained for $24 \mathrm{~h}$. The mixture was then allowed to cool to ambient temperature, quenched with saturated aqueous $\mathrm{NH}_{4} \mathrm{Cl}$, and extracted with $\mathrm{CH}_{2} \mathrm{Cl}_{2}$. The organic layer was dried over $\mathrm{Na}_{2} \mathrm{SO}_{4}$, filtered and concentrated in vacuo. The $\alpha, \alpha$-dibromoarylsilanes products were purified by flash chromatography on silica gel $(\mathrm{PE})(1.343 \mathrm{~g}$, yield $=82 \%)$.

Step 3: preparation of aroylsilane 1w

$\alpha, \alpha$-Dibromoarylsilane (1.0 equiv, $3.69 \mathrm{mmol}, 1.343 \mathrm{~g}$ ) was added to an aluminum foil-wrapped flask and dissolved in $\mathrm{H}_{2} \mathrm{O}$ /acetone/EtOH (in a ratio of 1:2:3 by volume) to make a $0.1 \mathrm{M}$ solution. 
$\mathrm{AgOAc}(2.0$ equiv, $7.38 \mathrm{mmol}, 1.23 \mathrm{~g}$ ) was added and the mixture was stirred for $24 \mathrm{~h}$ at room temperature. The mixture was filtered through a sand core funnel (G 4), concentrated in vacuo, diluted with brine, and then taken up with petroleum ether. The combined organic layers were dried over $\mathrm{Na}_{2} \mathrm{SO}_{4}$, and then concentrated in vacuo. The aroylsilane $\mathbf{1 w}$ was purified by flash chromatography on silica gel (PE:EA $=800: 1,568.6 \mathrm{mg}$, yield $=70 \%)$.

\section{Characterization Data of Aroylsilanes}<smiles>CS(=O)(=O)c1ccccc1</smiles>

\section{Phenyl(trimethylsilyl)methanone (1a) ${ }^{[1]}$}

Following the general procedure 1, aroylsilane 1a was obtained as a yellow oil by the purification with flash column chromatography on silica gel (PE:EA $=800: 1), 667.8 \mathrm{mg}$, yield $=75 \% .{ }^{1} \mathrm{H} \mathrm{NMR}$ (500 MHz, $\left.\mathrm{CDCl}_{3}\right): \delta=7.84-7.83(\mathrm{~m}, 2 \mathrm{H}), 7.54-7.51(\mathrm{~m}, 1 \mathrm{H}), 7.48-7.45(\mathrm{~m}, 2 \mathrm{H}), 0.38(\mathrm{~s}, 9 \mathrm{H})$. ${ }^{13} \mathrm{C}$ NMR $\left(125 \mathrm{~Hz}, \mathrm{CDCl}_{3}\right): \delta=237.3,142.9,134.3,130.3,129.1,0.2 . \mathrm{HRMS}$ (ESI-TOF) m/z: [M

$+\mathrm{H}]^{+}$Calcd for $\mathrm{C}_{10} \mathrm{H}_{15} \mathrm{OSi}$ 179.0887; Found 179.0880. FTIR $\left(\mathrm{KBr}, \mathrm{cm}^{-1}\right): 3742.06,3646.27$, 3665.42, 2923.36, 2351.40, 2326.17, 1656.07, 1409.35, 1025.23.

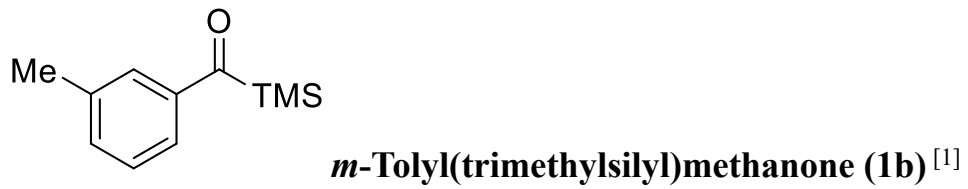

Following the general procedure 1, aroylsilane $\mathbf{1 b}$ was obtained as a yellow oil by the purification with flash column chromatography on silica gel $(\mathrm{PE}: \mathrm{EA}=800: 1), 576.3 \mathrm{mg}$, yield $=60 \% .{ }^{1} \mathrm{H} \mathrm{NMR}$ (500 MHz, $\left.\mathrm{CDCl}_{3}\right): \delta=7.66-7.64(\mathrm{~m}, 1 \mathrm{H}), 7.62(\mathrm{~s}, 1 \mathrm{H}), 7.37-7.33(\mathrm{~m}, 2 \mathrm{H}), 2.41(\mathrm{~s}, 3 \mathrm{H}), 0.37(\mathrm{~s}$, 9H). ${ }^{13} \mathrm{C} \mathrm{NMR}\left(125 \mathrm{~Hz}, \mathrm{CDCl}_{3}\right): \delta=244.1,143.9,137.6,133.9,132.5,131.4,127.2,22.5,0.2$. HRMS (ESI-TOF) m/z: [M + H] $]^{+}$Calcd for $\mathrm{C}_{11} \mathrm{H}_{17} \mathrm{OSi} 193.1043$; Found 193.1042. FTIR (KBr, cm 1): $3744.86,3649.53,3627.10,3178.50,2357.01,1406.54,1025.23,447.66$.

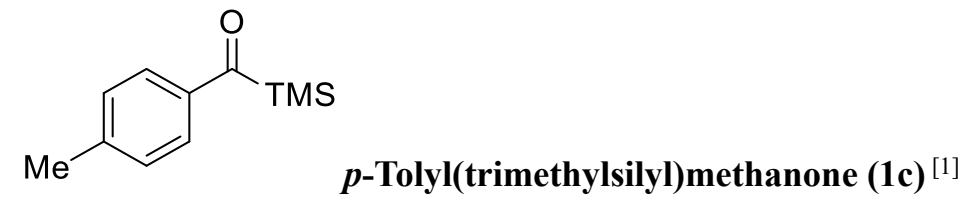

Following the general procedure 1, aroylsilane 1c was obtained as a yellow oil by the purification 
with flash column chromatography on silica gel $(\mathrm{PE}: \mathrm{EA}=800: 1), 595.5 \mathrm{mg}$, yield $=62 \% .{ }^{1} \mathrm{H}$ NMR $\left(500 \mathrm{MHz}, \mathrm{CDCl}_{3}\right): \delta=7.75(\mathrm{~d}, J=8.0 \mathrm{~Hz}, 2 \mathrm{H}), 7.26(\mathrm{~d}, J=8.0 \mathrm{~Hz}, 2 \mathrm{H}), 2.39(\mathrm{~s}, 3 \mathrm{H}), 0.37(\mathrm{~s}, 9 \mathrm{H})$. ${ }^{13} \mathrm{C}$ NMR $\left(125 \mathrm{~Hz}, \mathrm{CDCl}_{3}\right): \delta=236.3,145.0,140.7,130.8,129.2,23.2,0.2 . \mathrm{HRMS}(\mathrm{ESI}-\mathrm{TOF}) \mathrm{m} / \mathrm{z}$ : $[\mathrm{M}+\mathrm{H}]^{+}$Calcd for $\mathrm{C}_{11} \mathrm{H}_{17} \mathrm{OSi}$ 193.1043; Found 193.1043. FTIR $\left(\mathrm{KBr}, \mathrm{cm}^{-1}\right): 3747.66,3649.53$, $3565.42,3186.92,2354.21,1689.72,1406.54,666.36$.

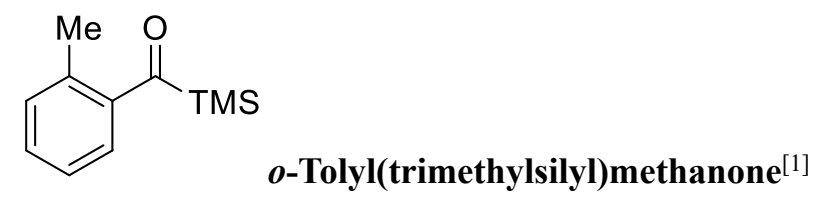

Following the general procedure 1, it was obtained as a yellow oil by the purification with flash column chromatography on silica gel $(\mathrm{PE}: \mathrm{EA}=800: 1), 432.2 \mathrm{mg}$, yield $=45 \% .{ }^{1} \mathrm{H} \mathrm{NMR}(500 \mathrm{MHz}$, $\left.\mathrm{CDCl}_{3}\right): \delta=7.57-7.56(\mathrm{~m}, 1 \mathrm{H}), 7.34-7.27(\mathrm{~m}, 2 \mathrm{H}), 7.22(\mathrm{~d}, J=7.5 \mathrm{~Hz}, 1 \mathrm{H}), 2.42(\mathrm{~s}, 3 \mathrm{H}), 0.32(\mathrm{~s}$, 9H). ${ }^{13} \mathrm{C} \mathrm{NMR}\left(125 \mathrm{~Hz}, \mathrm{CDCl}_{3}\right): \delta=244.1,143.9,137.6,133.9,132.5,131.4,127.2,22.5,0.2$. HRMS (ESI-TOF) m/z: [M + H] ${ }^{+}$Calcd for $\mathrm{C}_{11} \mathrm{H}_{17} \mathrm{OSi}$ 193.1043; Found 193.1035. FTIR (KBr, cm1): $3750.47,3627.10,3175.70,2354.21,2326.17,1647.66,1406.54,1025.23$.<smiles>CCc1ccc(C(=O)NS(C)(=O)=O)cc1</smiles>

(4-Ethylphenyl)(trimethylsilyl)methanone (1d)

Following the general procedure 1, aroylsilane 1d was obtained as a yellow oil by the purification with flash column chromatography on silica gel $(\mathrm{PE}: \mathrm{EA}=800: 1), 597.7 \mathrm{mg}$, yield $=58 \% .{ }^{1} \mathrm{H} \mathrm{NMR}$ $\left(500 \mathrm{MHz}, \mathrm{CDCl}_{3}\right): \delta=7.78(\mathrm{~d}, J=8.0 \mathrm{~Hz}, 2 \mathrm{H}), 7.30(\mathrm{~d}, J=8.0 \mathrm{~Hz}, 2 \mathrm{H}), 2.70(\mathrm{q}, J=15.5 \mathrm{~Hz}, 2 \mathrm{H})$, $1.26(\mathrm{t}, J=7.5 \mathrm{~Hz}, 3 \mathrm{H}), 0.37(\mathrm{~s}, 9 \mathrm{H}) .{ }^{13} \mathrm{C} \mathrm{NMR}\left(125 \mathrm{~Hz}, \mathrm{CDCl}_{3}\right): \delta=236.3,151.1,140.9,129.7$, 129.3, 30.5, 16.7, 0.2. HRMS (ESI-TOF) m/z: $[\mathrm{M}+\mathrm{H}]^{+}$Calcd for $\mathrm{C}_{12} \mathrm{H}_{19} \mathrm{OSi}$ 207.1200; Found 207.1204. FTIR (KBr, $\left.\mathrm{cm}^{-1}\right): 3739.25,3627.10,3565.42,2929.36,2362.62,2331.78,1656.07$, $1398.13,1025.23$<smiles>CC(=O)c1ccc(C(C)(C)C)cc1</smiles>

Following the general procedure 1, aroylsilane 1e was obtained as a yellow oil by the purification with flash column chromatography on silica gel $(\mathrm{PE}: \mathrm{EA}=800: 1), 702.4 \mathrm{mg}$, yield $=60 \% .{ }^{1} \mathrm{H}$ NMR 
$\left(500 \mathrm{MHz}, \mathrm{CDCl}_{3}\right): \delta=7.77(\mathrm{~d}, J=8.5 \mathrm{~Hz}, 2 \mathrm{H}), 7.28(\mathrm{~d}, J=8.0 \mathrm{~Hz}, 2 \mathrm{H}), 2.66(\mathrm{t}, J=8.0 \mathrm{~Hz}, 2 \mathrm{H})$, 1.65-1.59 (m, 2H), 1.38-1.34 (m, 2H), $0.93(\mathrm{t}, J=7.0 \mathrm{~Hz}, 3 \mathrm{H}), 0.37(\mathrm{~s}, 9 \mathrm{H}) .{ }^{13} \mathrm{C}$ NMR $(125$ $\left.\mathrm{Hz}, \mathrm{CDCl}_{3}\right): \delta=236.4,149.9,140.9,130.2,129.2,37.2,34.8,23.8,15.4,0.2$. HRMS (ESI-TOF) $\mathrm{m} / \mathrm{z}:[\mathrm{M}+\mathrm{H}]^{+}$Calcd for $\mathrm{C}_{14} \mathrm{H}_{23} \mathrm{OSi}$ 235.1513; Found 235.1515. FTIR $\left(\mathrm{KBr}, \mathrm{cm}^{-1}\right): 3739.25$, $3624.30,3565.42,3164.49,2359.81,2337.38,1653.27,1398.16,1019.63$.<smiles>CC(=O)c1ccc(C(C)C)cc1</smiles>

\section{(4-Isopropylphenyl)(trimethylsilyl)methanone (1f)}

Following the general procedure 1, aroylsilane $\mathbf{1 f}$ was obtained as a yellow oil by the purification with flash column chromatography on silica gel $(\mathrm{PE}: \mathrm{EA}=800: 1), 473.3 \mathrm{mg}$, yield $=43 \% .{ }^{1} \mathrm{H}$ NMR $\left(500 \mathrm{MHz}, \mathrm{CDCl}_{3}\right): \delta=7.79(\mathrm{~d}, J=8.5 \mathrm{~Hz}, 2 \mathrm{H}), 7.33(\mathrm{~d}, J=8.0 \mathrm{~Hz}, 2 \mathrm{H}), 2.99-2.93(\mathrm{~m}, 1 \mathrm{H}), 1.27$ $(\mathrm{d}, J=7.0 \mathrm{~Hz}, 6 \mathrm{H}), 0.37$ (s, 9H). ${ }^{13} \mathrm{C} \mathrm{NMR}\left(125 \mathrm{~Hz}, \mathrm{CDCl}_{3}\right): \delta=236.4,155.7,141.0,129.3,128.2$, 35.8, 25.1, 0.2. HRMS (ESI-TOF) m/z: $[\mathrm{M}+\mathrm{H}]^{+}$Calcd for $\mathrm{C}_{13} \mathrm{H}_{21} \mathrm{OSi} 221.1356$; Found 221.1358. FTIR $\left(\mathrm{KBr}, \mathrm{cm}^{-1}\right): 3742.32,3646.73,3178.50,2354.21,2331.78,1653.27,1560.75,1019.63$.<smiles>CC(C)(C)c1ccc(C(C)(C)C)cc1</smiles>

\section{(4-(Tert-butyl)phenyl)(trimethylsilyl)methanone (1g) ${ }^{[1]}$}

Following the general procedure 1, aroylsilane $1 \mathrm{~g}$ was obtained as a yellow oil by the purification with flash column chromatography on silica gel $(\mathrm{PE}: \mathrm{EA}=800: 1), 608.8 \mathrm{mg}$, yield $=52 \% .{ }^{1} \mathrm{H}$ NMR $\left(500 \mathrm{MHz}, \mathrm{CDCl}_{3}\right): \delta=7.81(\mathrm{~d}, J=8.0 \mathrm{~Hz}, 2 \mathrm{H}), 7.50(\mathrm{~d}, J=8.0 \mathrm{~Hz}, 2 \mathrm{H}), 1.34(\mathrm{~s}, 9 \mathrm{H}), 0.38(\mathrm{~s}, 9 \mathrm{H})$. ${ }^{13} \mathrm{C}$ NMR $\left(125 \mathrm{~Hz}, \mathrm{CDCl}_{3}\right): \delta=236.2,157.8,140.5,129.0,127.1,36.6,32.6,0.2 . \mathrm{HRMS}$ (ESI-TOF) m/z: $[\mathrm{M}+\mathrm{H}]^{+}$Calcd for $\mathrm{C}_{14} \mathrm{H}_{23} \mathrm{OSi}$ 235.1513; Found 235.1517. FTIR $\left(\mathrm{KBr}, \mathrm{cm}^{-1}\right)$ : 3747.66, $3652.34,3181.31,2923.36,2351.40,1653.27,1401.93,1019.63$.<smiles>COc1ccc(C(C)=O)cc1</smiles>

\section{(4-Methoxyphenyl)(trimethylsilyl)methanone (1h) ${ }^{[1]}$}

Following the general procedure 1, aroylsilane $\mathbf{1 h}$ was obtained as a yellow oil by the purification with flash column chromatography on silica gel $(\mathrm{PE}: \mathrm{EA}=800: 1), 677.3 \mathrm{mg}$, yield $=65 \% .{ }^{1} \mathrm{H} \mathrm{NMR}$ 
$\left(500 \mathrm{MHz}, \mathrm{CDCl}_{3}\right): \delta=7.86-7.84(\mathrm{~m}, 2 \mathrm{H}), 6.97-6.94(\mathrm{~m}, 2 \mathrm{H}), 3.85(\mathrm{~s}, 3 \mathrm{H}), 0.37(\mathrm{~s}, 9 \mathrm{H})$. ${ }^{13} \mathrm{C}$ NMR $\left(125 \mathrm{~Hz}, \mathrm{CDCl}_{3}\right): \delta=234.2,164.7,136.7,131.3,115.3,56.9,0.2 . \mathrm{HRMS}(\mathrm{ESI}-\mathrm{TOF}) \mathrm{m} / \mathrm{z}$ : $[\mathrm{M}+\mathrm{H}]^{+}$Calcd for $\mathrm{C}_{11} \mathrm{H}_{17} \mathrm{O}_{2} \mathrm{Si}$ 209.0992; Found 209.0992. FTIR $\left(\mathrm{KBr}, \mathrm{cm}^{-1}\right)$ : 3742.53, 3646.53, 3629.91, 3175.70, 2928.97, 2358.28, 2337.38, 1563.55, 1398.13, 1028.24.<smiles>CS(=O)(=O)c1ccc(OC(F)(F)F)cc1</smiles>

\section{(4-(Trifluoromethoxy)phenyl)(trimethylsilyl)methanone (1i)}

Following the general procedure 1, aroylsilane $\mathbf{1} \mathbf{i}$ was obtained as a yellow oil by the purification with flash column chromatography on silica gel $(\mathrm{PE}: \mathrm{EA}=800: 1), 773.1 \mathrm{mg}$, yield $=59 \% .{ }^{1} \mathrm{H}$ NMR $\left(500 \mathrm{MHz}, \mathrm{CDCl}_{3}\right): \delta=7.89(\mathrm{~d}, J=8.5 \mathrm{~Hz}, 2 \mathrm{H}), 7.31(\mathrm{~d}, J=8.5 \mathrm{~Hz}, 2 \mathrm{H}), 0.38(\mathrm{~s}, 9 \mathrm{H})$. ${ }^{13} \mathrm{C} \mathrm{NMR}\left(125 \mathrm{~Hz}, \mathrm{CDCl}_{3}\right): \delta=235.7,153.9\left(\mathrm{~d}, J_{\mathrm{C}-\mathrm{F}}=1.8 \mathrm{~Hz}\right), 141.1,131.1,122.3,122.0\left(\mathrm{q}, J_{\mathrm{C}-\mathrm{F}}\right.$ $=257.0 \mathrm{~Hz}$ ), 0.2. HRMS (ESI-TOF) $\mathrm{m} / \mathrm{z}:[\mathrm{M}+\mathrm{H}]^{+}$Calcd for $\mathrm{C}_{11} \mathrm{H}_{14} \mathrm{~F}_{3} \mathrm{O}_{2} \mathrm{Si}$ 263.0710; Found 263.0696. FTIR $\left(\mathrm{KBr}, \mathrm{cm}^{-1}\right): 3750.47,3627.10,3164.49,2362.62,2331.78,1656.07,1403.74$, 1019.63 .<smiles>CS(=O)(=O)c1ccc(F)cc1</smiles>

\section{(4-Fluorophenyl)(trimethylsilyl)methanone (1j) ${ }^{[5]}$}

Following the general procedure 1 , aroylsilane $\mathbf{1} \mathbf{j}$ was obtained as a yellow oil by the purification with flash column chromatography on silica gel $(\mathrm{PE}: \mathrm{EA}=800: 1), 558.8 \mathrm{mg}$, yield $=57 \% .{ }^{1} \mathrm{H}$ NMR $\left(500 \mathrm{MHz}, \mathrm{CDCl}_{3}\right): \delta=7.89-7.85(\mathrm{~m}, 2 \mathrm{H}), 7.15(\mathrm{~d}, J=8.5 \mathrm{~Hz}, 2 \mathrm{H}), 0.38(\mathrm{~s}, 9 \mathrm{H}) .{ }^{13} \mathrm{C}$ NMR $(125$ $\left.\mathrm{Hz}, \mathrm{CDCl}_{3}\right): \delta=235.1,167.0\left(\mathrm{~d}, J_{\mathrm{C}-\mathrm{F}}=252.8 \mathrm{~Hz}\right), 139.6\left(\mathrm{~d}, J_{\mathrm{C}-\mathrm{F}}=2.75 \mathrm{~Hz}\right), 131.6\left(\mathrm{~d}, J_{\mathrm{C}-\mathrm{F}}=9.13\right.$ $\mathrm{Hz}), 117.4\left(\mathrm{~d}, J_{\mathrm{C}-\mathrm{F}}=21.8 \mathrm{~Hz}\right)$, 0.2. HRMS (ESI-TOF) $\mathrm{m} / \mathrm{z}$ : $[\mathrm{M}+\mathrm{H}]^{+}$Calcd for $\mathrm{C}_{10} \mathrm{H}_{14} \mathrm{FOSi}$ 197.0792; Found 197.0787. FTIR $\left(\mathrm{KBr}, \mathrm{cm}^{-1}\right)$ : 3744.98, 3627.23, 2926.30, 2357.52, 1653.42, $1403.89,1019.63$<smiles>CS(=O)(=O)c1ccc(Cl)cc1</smiles>

\section{(4-Chlorophenyl)(trimethylsilyl)methanone $(1 \mathbf{k})^{[1]}$}

Following the general procedure 1, aroylsilane $1 \mathbf{k}$ was obtained as a yellow oil by the purification with flash column chromatography on silica gel $(\mathrm{PE}: \mathrm{EA}=800: 1), 371.1 \mathrm{mg}$, yield $=35 \% .{ }^{1} \mathrm{H}$ NMR 
$\left(500 \mathrm{MHz}, \mathrm{CDCl}_{3}\right): \delta=7.78-7.76(\mathrm{~m}, 2 \mathrm{H}), 7.46-7.44(\mathrm{~m}, 2 \mathrm{H}), 0.37(\mathrm{~s}, 9 \mathrm{H}) .{ }^{13} \mathrm{C} \mathrm{NMR}(125$ $\left.\mathrm{Hz}, \mathrm{CDCl}_{3}\right): \delta=235.9,141.2,140.7,130.7,130.5,0.2$. HRMS (ESI-TOF) m/z: $[\mathrm{M}+\mathrm{H}]^{+}$Calcd for $\mathrm{C}_{10} \mathrm{H}_{14} \mathrm{ClOSi}$ 213.0497; Found 213.0497. FTIR $\left(\mathrm{KBr}, \mathrm{cm}^{-1}\right)$ : 3742.49, 3645.44, 3181.31, 2354.21, 2328.97, 1665.27, 1400.93, 1020.87.<smiles>CS(=O)(=O)c1cccc(Cl)c1</smiles>

\section{(3-Chlorophenyl)(trimethylsilyl)methanone (11) ${ }^{[1]}$}

Following the general procedure 1, aroylsilane $\mathbf{1 l}$ was obtained as a yellow oil by the purification with flash column chromatography on silica gel (PE:EA $=800: 1), 498.3 \mathrm{mg}$, yield $=47 \% .{ }^{1} \mathrm{H}$ NMR $\left(500 \mathrm{MHz}, \mathrm{CDCl}_{3}\right): \delta=7.76-7.74(\mathrm{~m}, 1 \mathrm{H}), 7.73-7.70(\mathrm{~m}, 1 \mathrm{H}), 7.50-7.47(\mathrm{~m}, 1 \mathrm{H}), 7.44-7.39(\mathrm{~m}$, 1H), 0.38-0.37 (m, 9H). $\left.{ }^{13} \mathrm{C} \mathrm{NMR} \mathrm{(125} \mathrm{Hz,} \mathrm{CDCl}_{3}\right): \delta=236.0,144.3,136.8,134.2,131.7,128.6$, 127.7, 0.2. HRMS (ESI-TOF) m/z: $[\mathrm{M}+\mathrm{H}]^{+}$Calcd for $\mathrm{C}_{10} \mathrm{H}_{14} \mathrm{ClOSi} 213.0497$; Found 213.0497. FTIR $\left(\mathrm{KBr}, \mathrm{cm}^{-1}\right):$ 3749.45, 3743.10, 3647.37, 3209.95, 2354.21, 2331.78, 1651.73, 1557.50, $1393.67,1022.43$.<smiles>CS(=O)(=O)c1ccc(Br)cc1</smiles>

\section{(4-Bromophenyl)(trimethylsilyl)methanone $(1 \mathrm{~m})^{[1]}$}

Following the general procedure 1, aroylsilane 1m was obtained as a yellow oil by the purification with flash column chromatography on silica gel $(\mathrm{PE}: \mathrm{EA}=800: 1), 844.8 \mathrm{mg}$, yield $=66 \% .{ }^{1} \mathrm{H} \mathrm{NMR}$ $\left(500 \mathrm{MHz}, \mathrm{CDCl}_{3}\right): \delta=7.70-7.69(\mathrm{~m}, 2 \mathrm{H}), 7.63-7.61(\mathrm{~m}, 2 \mathrm{H}), 0.37(\mathrm{~s}, 9 \mathrm{H}) .{ }^{13} \mathrm{C}$ NMR $(125$ $\mathrm{Hz}, \mathrm{CDCl}_{3}$ ): $\delta=236.1,141.5,133.6,130.6,129.4,0.2$. HRMS (ESI-TOF) m/z: $[\mathrm{M}+\mathrm{H}]^{+}$Calcd for $\mathrm{C}_{10} \mathrm{H}_{14} \mathrm{BrOSi}$ 256.9992; Found 256.9990. FTIR (KBr, $\left.\mathrm{cm}^{-1}\right)$ : 3744.86, 3629.91, 3175.70, 2359.81, 2334.58, 1656.07, 1400.93, 1030.84, 671.96.<smiles>CS(=O)(=O)c1ccc(-c2ccccc2)cc1</smiles>

\section{(1,1'-Biphenyl)-4-yl(trimethylsilyl)methanone (1n) ${ }^{[6]}$}

Following the general procedure 1, aroylsilane 1n was obtained as a yellow solid by the purification with flash column chromatography on silica gel (PE:EA $=600: 1), 762.3 \mathrm{mg}$, yield $=60 \%$, m.p.: 73.4-73. ${ }^{\circ} \mathrm{C} .{ }^{1} \mathrm{H}$ NMR $\left(500 \mathrm{MHz}, \mathrm{CDCl}_{3}\right): \delta=7.92(\mathrm{~d}, J=8.0 \mathrm{~Hz}, 2 \mathrm{H}), 7.70(\mathrm{~d}, J=8.0 \mathrm{~Hz}, 2 \mathrm{H})$, 
$7.63(\mathrm{~d}, J=8.0 \mathrm{~Hz}, 2 \mathrm{H}), 7.46(\mathrm{t}, J=7.5 \mathrm{~Hz}, 2 \mathrm{H}), 7.39(\mathrm{t}, J=7.0 \mathrm{~Hz}, 1 \mathrm{H}), 0.41(\mathrm{~s}, 9 \mathrm{H}) .{ }^{13} \mathrm{C}$ NMR $(125$ $\left.\mathrm{Hz}, \mathrm{CDCl}_{3}\right): \delta=236.6,146.9,141.6,130.5,129.7,129.6,128.9,128.8,0.2 . \mathrm{HRMS}$ (ESI-TOF) m/z: $[\mathrm{M}+\mathrm{H}]^{+}$Calcd for $\mathrm{C}_{16} \mathrm{H}_{19} \mathrm{OSi}$ 255.1200; Found 255.1197. FTIR $\left(\mathrm{KBr}, \mathrm{cm}^{-1}\right)$ : 3750.47, 3629.91, $3555.42,3388.79,2920.56,2351.40,1653.27,1025.23$.<smiles>CC(=O)c1ccc2ccccc2c1</smiles>

\section{Naphthalen-2-yl(trimethylsilyl)methanone (10) ${ }^{[6]}$}

Following the general procedure 1, aroylsilane 10 was obtained as a yellow oil by the purification with flash column chromatography on silica gel $(\mathrm{PE}: \mathrm{EA}=800: 1), 342.1 \mathrm{mg}$, yield $=30 \% .{ }^{1} \mathrm{H}$ NMR $\left(500 \mathrm{MHz}, \mathrm{CDCl}_{3}\right): \delta=8.34(\mathrm{~s}, 1 \mathrm{H}), 7.95(\mathrm{~d}, J=8.0 \mathrm{~Hz}, 1 \mathrm{H}), 7.89-7.81(\mathrm{~m}, 3 \mathrm{H}), 7.56-7.49(\mathrm{~m}, 2 \mathrm{H})$, $0.42(\mathrm{~s}, 9 \mathrm{H}) .{ }^{13} \mathrm{C} \mathrm{NMR}\left(125 \mathrm{~Hz}, \mathrm{CDCl}_{3}\right): \delta=236.7,140.2,136.9,134.02,132.1,131.0,130.0,129.7$, 129.2, 128.1, 123.7, 0.2. HRMS (ESI-TOF) m/z: $[\mathrm{M}+\mathrm{H}]^{+}$Calcd for $\mathrm{C}_{14} \mathrm{H}_{17} \mathrm{OSi} 229.1043$; Found 229.1044. FTIR (KBr, $\left.\mathrm{cm}^{-1}\right): 3744.86,3627.10,3555.42,3198.13,2354.21,2340.19,1684.11$, $1403.74,1028.04$<smiles>CC(=O)c1cc(C)cc(C)c1</smiles>

(3,5-Dimethylphenyl)(trimethylsilyl)methanone (1p) ${ }^{[7]}$

Following the general procedure 1, aroylsilane $1 \mathbf{p}$ was obtained as a yellow oil by the purification with flash column chromatography on silica gel $(\mathrm{PE}: \mathrm{EA}=800: 1), 432.8 \mathrm{mg}$, yield $=42 \% .{ }^{1} \mathrm{H} \mathrm{NMR}$ $\left(500 \mathrm{MHz}, \mathrm{CDCl}_{3}\right): \delta=7.43(\mathrm{~s}, 2 \mathrm{H}), 7.17(\mathrm{~s}, 1 \mathrm{H}), 2.37$ (s, 6H), 0.37 (s, 9H). ${ }^{13} \mathrm{C}$ NMR $(125$ $\left.\mathrm{Hz}, \mathrm{CDCl}_{3}\right): \delta=237.5,143.2,139.7,135.8,126.8,22.7,0.2 . \mathrm{HRMS}(\mathrm{ESI}-\mathrm{TOF}) \mathrm{m} / \mathrm{z}:[\mathrm{M}+\mathrm{H}]^{+}$ Calcd for $\mathrm{C}_{12} \mathrm{H}_{19} \mathrm{OSi}$ 207.1200; Found 207.1202. FTIR (KBr, $\left.\mathrm{cm}^{-1}\right)$ : 3743.15, 3646.73, 3571.03, $3178.50,2357.01,2331.78,1651.75,1557.56,1392.52,1019.63$<smiles>CS(=O)(=O)c1ccc2c(c1)OCO2</smiles>

\section{Benzo[d] [1,3]dioxol-5-yl(trimethylsilyl)methanone (1q) ${ }^{[8]}$}

Following the general procedure 1, aroylsilane 1q was obtained as a yellow oil by the purification with flash column chromatography on silica gel $(\mathrm{PE}: \mathrm{EA}=600: 1), 166.6 \mathrm{mg}$, yield $=15 \% .{ }^{1} \mathrm{H}$ NMR $\left(500 \mathrm{MHz}, \mathrm{CDCl}_{3}\right): \delta=7.49(\mathrm{dd}, J=8.0 \mathrm{~Hz}, J=1.5 \mathrm{~Hz}, 1 \mathrm{H}), 7.28(\mathrm{~d}, J=1.5 \mathrm{~Hz}, 1 \mathrm{H}), 6.87(\mathrm{~d}, J=$ 
$8.0 \mathrm{~Hz}, 1 \mathrm{H}), 6.02(\mathrm{~s}, 2 \mathrm{H}), 0.36(\mathrm{~d}, J=0.5 \mathrm{~Hz}, 9 \mathrm{H}) .{ }^{13} \mathrm{C} \mathrm{NMR}\left(125 \mathrm{~Hz}, \mathrm{CDCl}_{3}\right): \delta=33.4,152.9$, $149.8,138.5,127.0,109.2,107.2,103.1,0.2$. HRMS (ESI-TOF) $\mathrm{m} / \mathrm{z}:[\mathrm{M}+\mathrm{H}]^{+}$Calcd for $\mathrm{C}_{11} \mathrm{H}_{15} \mathrm{O}_{3} \mathrm{Si}$ 223.0785; Found 223.0780. FTIR (KBr, $\left.\mathrm{cm}^{-1}\right)$ : 3747.66, 3627.07, 3198.13, 2354.21, $2331.78,1656.07,1400.93,1030.84$.<smiles>COc1ccc(C(C)=O)cc1F</smiles>

\section{(3-Fluoro-4-methoxyphenyl)(trimethylsilyl)methanone (1r)}

Following the general procedure 1, aroylsilane $1 \mathbf{r}$ was obtained as a yellow oil by the purification with flash column chromatography on silica gel $(\mathrm{PE}: \mathrm{EA}=600: 1), 350.4 \mathrm{mg}$, yield $=31 \% .{ }^{1} \mathrm{H} \mathrm{NMR}$ $\left(500 \mathrm{MHz}, \mathrm{CDCl}_{3}\right): \delta=7.68-7.67(\mathrm{~m}, 1 \mathrm{H}), 7.56(\mathrm{dd}, J=11.5 \mathrm{~Hz}, J=2.0 \mathrm{~Hz}, 1 \mathrm{H}), 7.04(\mathrm{t}, J=8.5$ $\mathrm{Hz}, 1 \mathrm{H}), 3.95$ (s, 3H), 0.37 (s, 9H). ${ }^{13} \mathrm{C}$ NMR $\left(125 \mathrm{~Hz}, \mathrm{CDCl}_{3}\right): \delta=233.6,153.9\left(\mathrm{~d}, J_{\mathrm{C}-\mathrm{F}}=247.4\right.$ $\mathrm{Hz}), 153.2\left(\mathrm{~d}, J_{\mathrm{C}-\mathrm{F}}=11.0 \mathrm{~Hz}\right), 136.7\left(\mathrm{~d}, J_{\mathrm{C}-\mathrm{F}}=3.5 \mathrm{~Hz}\right), 127.4\left(\mathrm{~d}, J_{\mathrm{C}-\mathrm{F}}=3.3 \mathrm{~Hz}\right), 115.4\left(\mathrm{~d}, J_{\mathrm{C}-\mathrm{F}}=\right.$ $17.9 \mathrm{~Hz}), 113.7\left(\mathrm{~d}, J_{\mathrm{C}-\mathrm{F}}=1.4 \mathrm{~Hz}\right), 57.8,0.2 . \mathrm{HRMS}\left(\right.$ ESI-TOF) $\mathrm{m} / \mathrm{z}:[\mathrm{M}+\mathrm{H}]^{+}$Calcd for $\mathrm{C}_{11} \mathrm{H}_{16} \mathrm{FO}_{2} \mathrm{Si}$ 227.0898; Found 227.0898. FTIR $\left(\mathrm{KBr}, \mathrm{cm}^{-1}\right)$ : 3747.66, 3649.53, 3568.22, 3181.31, 2354.21, 2331.78, 1560.75, 1395.99, 1025.23.<smiles>CC(=O)c1ccc(F)c(C)c1</smiles>

\section{(4-Fluoro-3-methylphenyl)(trimethylsilyl)methanone (1s)}

Following the general procedure 1, aroylsilane 1s was obtained as a yellow oil by the purification with flash column chromatography on silica gel $(\mathrm{PE}: \mathrm{EA}=800: 1), 367.7 \mathrm{mg}$, yield $=35 \% .{ }^{1} \mathrm{H}$ NMR $\left(500 \mathrm{MHz}, \mathrm{CDCl}_{3}\right): \delta=7.71-7.69(\mathrm{~m}, 2 \mathrm{H}), 7.07(\mathrm{t}, J=9.0 \mathrm{~Hz}, 1 \mathrm{H}), 2.32(\mathrm{~d}, J=2.0 \mathrm{~Hz}, 3 \mathrm{H}), 0.38$ (s, 9H). ${ }^{13} \mathrm{C} \mathrm{NMR}\left(125 \mathrm{~Hz}, \mathrm{CDCl}_{3}\right): \delta=235.1,165.6\left(\mathrm{~d}, J_{\mathrm{C}-\mathrm{F}}=251.8 \mathrm{~Hz}\right), 139.4\left(\mathrm{~d}, J_{\mathrm{C}-\mathrm{F}}=3.1 \mathrm{~Hz}\right)$, $132.3\left(\mathrm{~d}, J_{\mathrm{C}-\mathrm{F}}=6.5 \mathrm{~Hz}\right), 129.4\left(\mathrm{~d}, J_{\mathrm{C}-\mathrm{F}}=9.1 \mathrm{~Hz}\right), 127.0\left(\mathrm{~d}, J_{\mathrm{C}-\mathrm{F}}=17.8 \mathrm{~Hz}\right), 116.8\left(\mathrm{~d}, J_{\mathrm{C}-\mathrm{F}}=22.9 \mathrm{~Hz}\right)$, $16.1\left(\mathrm{~d}, J_{\mathrm{C}-\mathrm{F}}=3.4 \mathrm{~Hz}\right), 0.2 . \mathrm{HRMS}(\mathrm{ESI}-\mathrm{TOF}) \mathrm{m} / \mathrm{z}:[\mathrm{M}+\mathrm{H}]^{+}$Calcd for $\mathrm{C}_{11} \mathrm{H}_{16} \mathrm{FOSi} 211.0949$; Found 211.0948. FTIR $\left(\mathrm{KBr}, \mathrm{cm}^{-1}\right): 3744.86,3629.91,3565.42,2362.62,2331.78,1653.27$, $1557.94,1406.54,1021.86$. 
TMS

\section{(4-((1s,4r)-4-Butylcyclohexyl)phenyl)(trimethylsilyl)methanone (1t)}

Following the general procedure 1, aroylsilane $1 \mathbf{t}$ was obtained as a yellow oil by the purification with flash column chromatography on silica gel $(\mathrm{PE}: \mathrm{EA}=800: 1), 743.1 \mathrm{mg}$, yield $=47 \% .{ }^{1} \mathrm{H}$ NMR $\left(500 \mathrm{MHz}, \mathrm{CDCl}_{3}\right): \delta=7.78(\mathrm{~d}, J=8.0 \mathrm{~Hz}, 2 \mathrm{H}), 7.31(\mathrm{~d}, J=8.0 \mathrm{~Hz}, 2 \mathrm{H}), 2.52(\mathrm{tt}, J=12.0 \mathrm{~Hz}, J=$ $3.0 \mathrm{~Hz}, 1 \mathrm{H}), 1.90-1.88(\mathrm{~m}, 4 \mathrm{H}), 1.50-1.42(\mathrm{~m}, 2 \mathrm{H}), 1.32-1.23(\mathrm{~m}, 7 \mathrm{H}), 1.10-1.02(\mathrm{~m}, 2 \mathrm{H}), 0.92-0.89$ $(\mathrm{m}, 3 \mathrm{H}), 0.37(\mathrm{~s}, 9 \mathrm{H}) .{ }^{13} \mathrm{C} \mathrm{NMR}\left(125 \mathrm{~Hz}, \mathrm{CDCl}_{3}\right): \delta=236.4,154.7,141.0,129.3,128.6,46.3,38.7$, 38.6, 35.5, 34.9, 30.7, 24.5, 15.7, 0.2. HRMS (ESI-TOF) $\mathrm{m} / \mathrm{z}:[\mathrm{M}+\mathrm{H}]^{+}$Calcd for $\mathrm{C}_{20} \mathrm{H}_{33} \mathrm{OSi}$ 317.2295; Found 317.2305. FTIR (KBr, $\left.\mathrm{cm}^{-1}\right)$ : 3742.06, 3571.09, 3181.31, 2931.78, 2357.01, $2334.58,1560.75,1403.74$<smiles>CC(=O)c1ccccc1</smiles>

\section{(Dimethyl(phenyl)silyl)(phenyl)methanone (1u) ${ }^{[2]}$}

Following the general procedure 2, aroylsilane 1u was obtained as a yellow oil by the purification with flash column chromatography on silica gel $(\mathrm{PE}: \mathrm{EA}=800: 1), 151.3 \mathrm{mg}$, yield $=42 \% .{ }^{1} \mathrm{H}$ NMR $\left(500 \mathrm{MHz}, \mathrm{CDCl}_{3}\right): \delta=7.48-746(\mathrm{~m}, 2 \mathrm{H}), 7.32-7.30(\mathrm{~m}, 2 \mathrm{H}), 7.17-7.15(\mathrm{~m}, 1 \mathrm{H}), 7.12-7.7 .06(\mathrm{~m}$, 5H), $0.34(\mathrm{~s}, 6 \mathrm{H}) .{ }^{13} \mathrm{C} \mathrm{NMR}\left(125 \mathrm{~Hz}, \mathrm{CDCl}_{3}\right): \delta=236.8,144.4,138.8,137.1,135.9,132.9,131.7$, 131.4, 130.9, 0.2. HRMS (ESI-TOF) m/z: [M + H] ${ }^{+}$Calcd for $\mathrm{C}_{15} \mathrm{H}_{17} \mathrm{OSi} 241.1043$; Found 241.1049. FTIR $\left(\mathrm{KBr}, \mathrm{cm}^{-1}\right)$ : 3744.86, 3629.91, 3360.75, 3175.70, 2928.97, 2354.21, 1656.07, 1406.54, 1025.23

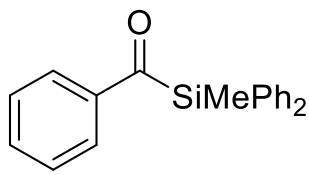

\section{(Methyldiphenylsilyl)(phenyl)methanone (1v) ${ }^{[2]}$}

Following the general procedure 2, aroylsilane $1 \mathbf{v}$ was obtained as a yellow oil by the purification with flash column chromatography on silica gel $(\mathrm{PE}: \mathrm{EA}=800: 1), 226.6 \mathrm{mg}$, yield $=50 \% .{ }^{1} \mathrm{H}$ NMR $\left(500 \mathrm{MHz}, \mathrm{CDCl}_{3}\right): \delta=7.78-7.76(\mathrm{~m}, 2 \mathrm{H}), 7.61-7.58(\mathrm{~m}, 4 \mathrm{H}), 7.46-7.31(\mathrm{~m}, 9 \mathrm{H}), 0.87-0.86(\mathrm{~m}$, 3H). ${ }^{13} \mathrm{C}$ NMR $\left(125 \mathrm{~Hz}, \mathrm{CDCl}_{3}\right): \delta=235.5,145.3,138.6,137.3,136.4,133.5,132.0,131.7,131.7$, 
0.2. HRMS (ESI-TOF) m/z: $[\mathrm{M}+\mathrm{H}]^{+}$Calcd for $\mathrm{C}_{20} \mathrm{H}_{19} \mathrm{OSi}$ 303.1200; Found 303.1198. FTIR (KBr, $\left.\mathrm{cm}^{-1}\right): 3744.86,3624.30,3565.42,3172.90,2348.60,1650.47,1402.86,1022.43$.<smiles>CCSC(=O)c1ccccc1</smiles>

\section{Phenyl(triethylsilyl)methanone (1w) ${ }^{[3]}$}

Following the general procedure 3, aroylsilane $1 \mathbf{w}$ was obtained as a yellow oil by the purification with flash column chromatography on silica gel $(\mathrm{PE}: \mathrm{EA}=800: 1), 568.6 \mathrm{mg}$, yield $=47 \% .{ }^{1} \mathrm{H}$ NMR $\left(500 \mathrm{MHz}, \mathrm{CDCl}_{3}\right): \delta=7.81-7.80(\mathrm{~m}, 2 \mathrm{H}), 7.54-7.51(\mathrm{~m}, 1 \mathrm{H}), 7.48-7.45(\mathrm{~m}, 2 \mathrm{H}), 1.01-0.98(\mathrm{~m}$, 9H), 0.94-0.89 (m, 6H). ${ }^{13} \mathrm{C} \mathrm{NMR}\left(125 \mathrm{~Hz}, \mathrm{CDCl}_{3}\right): \delta=232.6,139.0,129.2,125.2,123.6,4.0,0.2$. HRMS (ESI-TOF) m/z: [M + H] $]^{+}$Calcd for $\mathrm{C}_{13} \mathrm{H}_{21} \mathrm{OSi}$ 221.1356; Found 221.1366. FTIR (KBr, cm1): 3742.20, 3667.44, 3626.50, 3144.86, 2357.01, 2331.78, 1557.50, 1403.09, 1025.23.

\section{General Procedure for the Ru-Catalyzed Synthesis of Silyloxy Indenes}

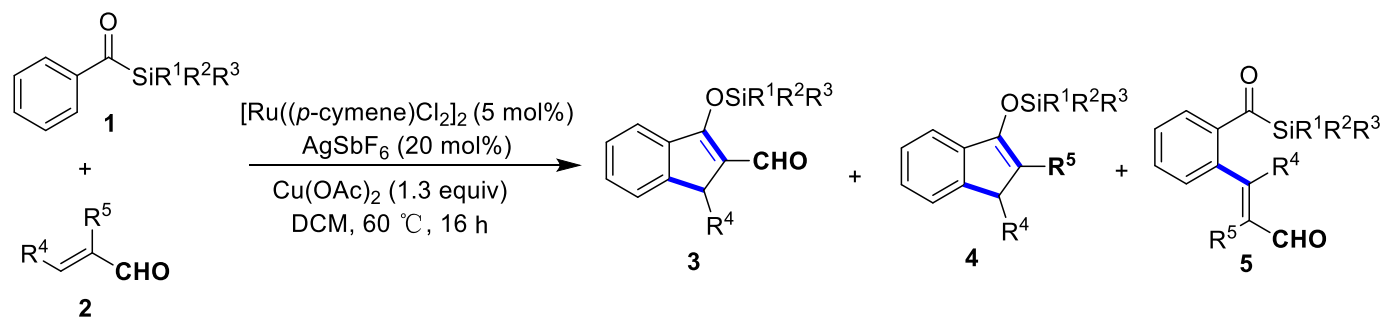

A screw-cap vial was charged with $\left[\mathrm{Ru}(p-c y m e n e) \mathrm{Cl}_{2}\right]_{2}(5 \mathrm{~mol} \%, 0.01 \mathrm{mmol}), \mathrm{AgSbF}_{6}(20$ $\mathrm{mol} \%, 0.04 \mathrm{mmol}), \mathrm{Cu}(\mathrm{OAc})_{2}(1.3$ equiv, $0.26 \mathrm{mmol})$ and $\mathrm{DCM}(1.0 \mathrm{~mL})$. Then, aroylsilane $1(1.0$ equiv, $0.2 \mathrm{mmol}$ ) and acrolein 2 (3.0 equiv, $0.6 \mathrm{mmol}$ ) were added into the solution in sequence. The vial was sealed under argon and heated to $60^{\circ} \mathrm{C}$ in an oil bath with stirring for $16 \mathrm{~h}$. After cooling down, the mixture was directly applied to a flash column chromatography on silica gel for separation using a mixed PE and EA. 


\section{Characterization Data of Silyloxy Indenes Products}

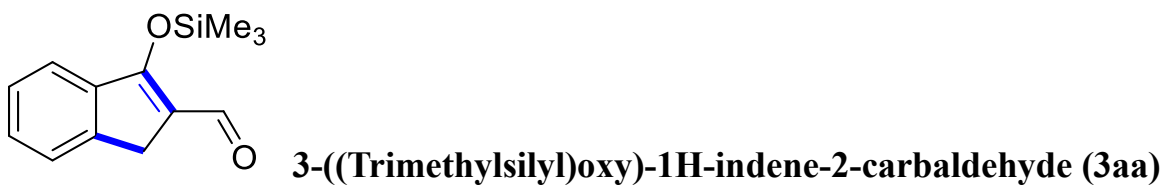

Following the general procedure, 3aa was obtained as a pale yellow solid by the purification with flash column chromatography on silica gel $(\mathrm{PE}: \mathrm{EA}=160: 1)$, m.p.: $60.5-62.8{ }^{\circ} \mathrm{C}, 33.9 \mathrm{mg}$, yield $=$ 73\%. ${ }^{1} \mathrm{H}$ NMR (500 MHz, $\left.\mathrm{CDCl}_{3}\right): \delta=10.26(\mathrm{~s}, 1 \mathrm{H}), 7.77-7.73(\mathrm{~m}, 1 \mathrm{H})$, 7.56-7.54 (m, 1H), 7.38$7.33(\mathrm{~m}, 2 \mathrm{H}), 3.72(\mathrm{~s}, 2 \mathrm{H}), 0.51(\mathrm{~s}, 9 \mathrm{H}) .{ }^{13} \mathrm{C} \mathrm{NMR}\left(125 \mathrm{~Hz}, \mathrm{CDCl}_{3}\right): \delta=188.6,160.8,153.6,146.3$, 143.5, 127.0, 125.6, 124.2, 123.5, 37.3, 0.2. HRMS (ESI-TOF) m/z: $[\mathrm{M}+\mathrm{H}]^{+}$Calcd for $\mathrm{C}_{13} \mathrm{H}_{17} \mathrm{O}_{2} \mathrm{Si}$ 233.0992; Found 233.0990. FTIR (KBr, $\left.\mathrm{cm}^{-1}\right)$ : 3450.19, 3410.97, 2956.89, 1643.81, 1658.52, $1538.35,1402.49,844.89$.

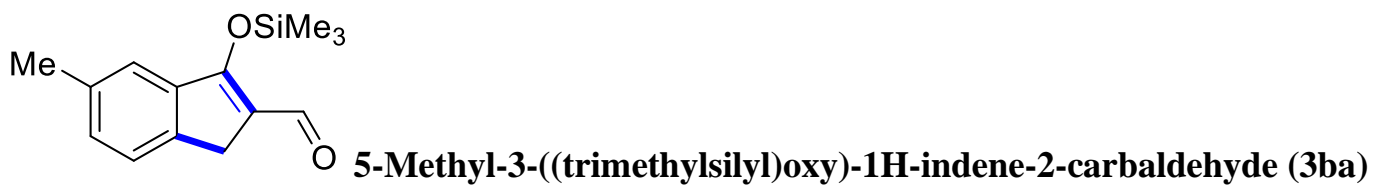

Following the general procedure, 3ba was obtained as a pale yellow solid by the purification with flash column chromatography on silica gel $(\mathrm{PE}: \mathrm{EA}=160: 1)$, m.p.: $57.3-59.9^{\circ} \mathrm{C}, 33.5 \mathrm{mg}$, yield $=$ 68\%. ${ }^{1} \mathrm{H}$ NMR (500 MHz, $\left.\mathrm{CDCl}_{3}\right): \delta=10.24(\mathrm{~s}, 1 \mathrm{H}), 7.54(\mathrm{~s}, 1 \mathrm{H}), 7.44(\mathrm{~d}, J=7.5 \mathrm{~Hz}, 1 \mathrm{H}), 7.19$ $(\mathrm{d}, J=7.5 \mathrm{~Hz}, 1 \mathrm{H}), 3.68(\mathrm{~s}, 2 \mathrm{H}), 2.44(\mathrm{~s}, 3 \mathrm{H}), 0.51(\mathrm{~s}, 9 \mathrm{H}) .{ }^{13} \mathrm{C} \mathrm{NMR}\left(125 \mathrm{~Hz}, \mathrm{CDCl}_{3}\right): \delta=188.6$, $160.8,153.8,146.5,140.6,135.1,128.0,124.7,123.1,36.8,20.5,0.2$. HRMS (ESI-TOF) m/z: [M $+\mathrm{H}]^{+}$Calcd for $\mathrm{C}_{14} \mathrm{H}_{19} \mathrm{O}_{2} \mathrm{Si}$ 247.1149; Found 247.1156. FTIR (KBr, $\left.\mathrm{cm}^{-1}\right)$ : 3444.57, 3176.13, $1659.72,1651.56,1455.14,1402.44,1253.81,1015.45,840.67,802.07$.

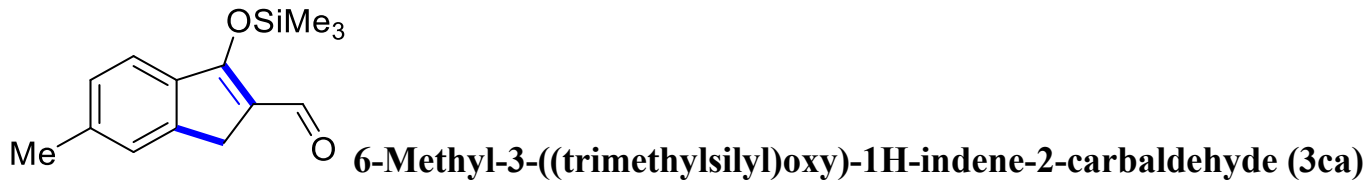

Following the general procedure, 3ca was obtained as a pale yellow solid by the purification with flash column chromatography on silica gel $(\mathrm{PE}: \mathrm{EA}=160: 1)$, m.p.: 77.4-78.2 ${ }^{\circ} \mathrm{C}, 25.6 \mathrm{mg}$, yield $=$ 52\%. ${ }^{1} \mathrm{H}$ NMR (500 MHz, $\left.\mathrm{CDCl}_{3}\right): \delta=10.22(\mathrm{~s}, 1 \mathrm{H}), 7.63(\mathrm{~d}, J=8.0 \mathrm{~Hz}, 1 \mathrm{H}), 7.38(\mathrm{~s}, 1 \mathrm{H}), 7.17$ $(\mathrm{d}, J=8.0 \mathrm{~Hz}, 1 \mathrm{H}), 3.68(\mathrm{~s}, 2 \mathrm{H}), 2.42(\mathrm{~s}, 3 \mathrm{H}), 0.49(\mathrm{~s}, 9 \mathrm{H}) .{ }^{13} \mathrm{C} \mathrm{NMR}\left(125 \mathrm{~Hz}, \mathrm{CDCl}_{3}\right): \delta=188.5$, 
161.1, 152.8, 144.0, 143.8, 137.5, 126.6, 124.2, 124.0, 37.0, 20.5, 0.2. HRMS (ESI-TOF) m/z: [M

$+\mathrm{H}]^{+}$Calcd for $\mathrm{C}_{14} \mathrm{H}_{19} \mathrm{O}_{2} \mathrm{Si}$ 247.1149; Found 247.1149. FTIR $\left(\mathrm{KBr}, \mathrm{cm}^{-1}\right)$ : 3507.43, 3441.30, $3175.56,3144.15,1651.19,1644.34,1402.01,1247.33,844.90$.<smiles>CCOC1=C(C=O)Cc2cc(CC)ccc21</smiles>

6-Ethyl-3-((trimethylsilyl)oxy)-1H-indene-2-carbaldehyde (3da)

Following the general procedure, 3da was obtained as a pale yellow solid by the purification with flash column chromatography on silica gel $(\mathrm{PE}: \mathrm{EA}=160: 1)$, m.p.: $60.0-60.8^{\circ} \mathrm{C}, 32.3 \mathrm{mg}$, yield $=$ 62\%. ${ }^{1} \mathrm{H}$ NMR (500 MHz, $\left.\mathrm{CDCl}_{3}\right): \delta=10.22(\mathrm{~s}, 1 \mathrm{H}), 7.66(\mathrm{~d}, J=8.0 \mathrm{~Hz}, 1 \mathrm{H}), 7.40(\mathrm{~s}, 1 \mathrm{H}), 7.19$ (d, $J=8.0 \mathrm{~Hz}, 1 \mathrm{H}), 3.69$ (s, 2H), 2.74-2.69 (q, $J=7.5 \mathrm{~Hz}, 2 \mathrm{H}), 1.27$ (t, $J=7.5 \mathrm{~Hz}, 3 \mathrm{H}), 0.50$ (s, 1H). ${ }^{13} \mathrm{C}$ NMR $\left(125 \mathrm{~Hz}, \mathrm{CDCl}_{3}\right): \delta=188.5,161.1,153.0,144.1,144.0,143.9,125.5,124.1,123.0,37.02$, 27.9, 14.6, 0.2. HRMS (ESI-TOF) m/z: $[\mathrm{M}+\mathrm{H}]^{+}$Calcd for $\mathrm{C}_{15} \mathrm{H}_{21} \mathrm{O}_{2} \mathrm{Si} 261.1305$; Found 261.1300. FTIR (KBr, $\left.\mathrm{cm}^{-1}\right): 3444.29,3417.44,3168.01,2966.48,1651.10,1402.79,1246.27,843.90$.<smiles>CCc1ccc(C(C)=O)c(/C=C/C=O)c1</smiles>

(E)-3-(5-ethyl-2-((trimethylsilyl)carbonyl)phenyl)acrylaldehyde

(5da)

Following the general procedure, $\mathbf{3 d a}$ was obtained as a brown oil by the purification with flash column chromatography on silica gel $(\mathrm{PE}: \mathrm{EA}=50: 1), 4.2 \mathrm{mg}$, yield $=8 \% .{ }^{1} \mathrm{H}$ NMR $(500 \mathrm{MHz}$, $\left.\mathrm{CDCl}_{3}\right): \delta=9.74(\mathrm{~d}, J=8.0 \mathrm{~Hz}, 1 \mathrm{H}), 8.00(\mathrm{~d}, J=16.0 \mathrm{~Hz}, 1 \mathrm{H}), 7.73(\mathrm{~d}, J=8.0 \mathrm{~Hz}, 1 \mathrm{H}), 7.45(\mathrm{~s}$, 1H), $7.39(\mathrm{~d}, J=8.0 \mathrm{~Hz}, 1 \mathrm{H}), 6.57(\mathrm{q}, J=8.0 \mathrm{~Hz}, 1 \mathrm{H}), 2.74(\mathrm{q}, J=7.5 \mathrm{~Hz}, 2 \mathrm{H}), 1.29$ (t, $J=7.5 \mathrm{~Hz}$, $3 \mathrm{H}), 1.26$ (s, 2H), 0.36 (s, 9H). ${ }^{13} \mathrm{C} \mathrm{NMR}\left(125 \mathrm{~Hz}, \mathrm{CDCl}_{3}\right): \delta=196.0,154.3,150.2,141.1,134.2$, 132.8, 132.4, 131.3, 129.7, 30.5, 16.8, 0.2. HRMS (ESI-TOF) m/z: $[\mathrm{M}+\mathrm{H}]^{+}$Calcd for $\mathrm{C}_{15} \mathrm{H}_{21} \mathrm{O}_{2} \mathrm{Si}$ 261.1305; Found 261.1310. FTIR (KBr, $\left.\mathrm{cm}^{-1}\right)$ : 3444.47, 3416.34, 3171.24, 2923.44, 2358.73, $1643.03,1632.00,1401.94$.<smiles>COC1=C(C=O)Cc2cc(C(C)(C)C)ccc21</smiles>

6-Butyl-3-((trimethylsilyl)oxy)-1H-indene-2-carbaldehyde (3ea) 
Following the general procedure, 3ea was obtained as a yellow oil by the purification with flash column chromatography on silica gel $(\mathrm{PE}: \mathrm{EA}=160: 1), 37.5 \mathrm{mg}$, yield $=65 \% .{ }^{1} \mathrm{H} \mathrm{NMR}(500 \mathrm{MHz}$, $\left.\mathrm{CDCl}_{3}\right): \delta=10.22(\mathrm{~s}, 1 \mathrm{H}), 7.65(\mathrm{~d}, J=8.0 \mathrm{~Hz}, 1 \mathrm{H}), 7.38(\mathrm{~s}, 1 \mathrm{H}), 7.17(\mathrm{dd}, J=8.0 \mathrm{~Hz}, J=1.5 \mathrm{~Hz}$, 1H), $3.69(\mathrm{~s}, 2 \mathrm{H}), 2.67$ (t, $J=7.5 \mathrm{~Hz}, 2 \mathrm{H}), 1.66-1.60(\mathrm{~m}, 2 \mathrm{H}), 1.41-1.35(\mathrm{~m}, 2 \mathrm{H}), 0.94(\mathrm{t}, J=7.5$ $\mathrm{Hz}, 3 \mathrm{H}), 0.50$ (s, 9H). ${ }^{13} \mathrm{C} \mathrm{NMR}\left(125 \mathrm{~Hz}, \mathrm{CDCl}_{3}\right): \delta=188.5,161.1,152.9,144.0,143.9,142.6$, 126.0, 124.0, 123.5, 37.0, 34.7, 32.6, 21.3, 12.8, 0.2. HRMS (ESI-TOF) $\mathrm{m} / \mathrm{z}:[\mathrm{M}+\mathrm{H}]^{+}$Calcd for $\mathrm{C}_{17} \mathrm{H}_{25} \mathrm{O}_{2} \mathrm{Si}$ 289.1618; Found 289.1619. FTIR (KBr, $\left.\mathrm{cm}^{-1}\right)$ : 3472.50, 3444.54, 3417.37, 3175.09, $1651.34,1644.55,1504.69,1402.46$.<smiles>COC1=C(C=O)Cc2cc(C(C)C)ccc21</smiles>

6-iso-Propyl-3-((trimethylsilyl)oxy)-1H-indene-2-carbaldehyde

(3fa)

Following the general procedure, $\mathbf{3 f a}$ was obtained as a pale yellow solid by the purification with flash column chromatography on silica gel $(\mathrm{PE}: \mathrm{EA}=160: 1)$, m.p.: 50.2-52.4 ${ }^{\circ} \mathrm{C}, 36.7 \mathrm{mg}$, yield $=$ 67\%. ${ }^{1} \mathrm{H}$ NMR (500 MHz, $\left.\mathrm{CDCl}_{3}\right): \delta=10.23(\mathrm{~d}, J=3.5 \mathrm{~Hz}, 1 \mathrm{H}), 7.68(\mathrm{dd}, J=8.5 \mathrm{~Hz}, J=3.0 \mathrm{~Hz}$, 1H), $7.43(\mathrm{~s}, 1 \mathrm{H}), 7.23(\mathrm{~d}, J=8.0 \mathrm{~Hz}, 1 \mathrm{H}), 3.70(\mathrm{~d}, J=2.0 \mathrm{~Hz}, 2 \mathrm{H}), 3.02-2.93(\mathrm{~m}, 1 \mathrm{H}), 1.29(\mathrm{dd}, J$ $=7.0 \mathrm{~Hz}, J=2.0 \mathrm{~Hz}, 6 \mathrm{H}), 0.50(\mathrm{~d}, J=2.5 \mathrm{~Hz}, 9 \mathrm{H}) .{ }^{13} \mathrm{C} \mathrm{NMR}\left(125 \mathrm{~Hz}, \mathrm{CDCl}_{3}\right): \delta=188.5,161.1$, 153.0, 148.6, 144.2, 144.0, 124.1, 124.1, 121.5, 37.1, 33.2, 22.9, 0.2. HRMS (ESI-TOF) m/z: [M + $\mathrm{H}]^{+}$Calcd for $\mathrm{C}_{16} \mathrm{H}_{23} \mathrm{O}_{2} \mathrm{Si} 275.1462$; Found 275.1459. FTIR $\left(\mathrm{KBr}, \mathrm{cm}^{-1}\right): 3452.60,3417.27,2958.38$, $1658.87,1402.63,1384.70,1251.86,841.72$.<smiles>COC1=C(C=O)Cc2cc(C(C)(C)C)ccc21</smiles>
6-(tert-Butyl)-3-((trimethylsilyl)oxy)-1H-indene-2-carbaldehyde

(3ga)

Following the general procedure, 3ga was obtained as a pale yellow solid by the purification with flash column chromatography on silica gel (PE:EA = 160:1), m.p.: $66.1-66.8^{\circ} \mathrm{C}, 35.2 \mathrm{mg}$, yield $=$ 
61\%. ${ }^{1} \mathrm{H}$ NMR (500 MHz, $\left.\mathrm{CDCl}_{3}\right): \delta=10.23(\mathrm{~s}, 1 \mathrm{H}), 7.69(\mathrm{~d}, J=8.0 \mathrm{~Hz}, 1 \mathrm{H}), 7.60(\mathrm{~d}, J=0.5 \mathrm{~Hz}$, 1H), $7.41(\mathrm{dd}, J=8.0 \mathrm{~Hz}, J=1.5 \mathrm{~Hz}, 1 \mathrm{H}), 3.71(\mathrm{~s}, 2 \mathrm{H}), 1.36(\mathrm{~s}, 9 \mathrm{H}), 0.50(\mathrm{~s}, 9 \mathrm{H}) .{ }^{13} \mathrm{C}$ NMR $(125$ $\left.\mathrm{Hz}, \mathrm{CDCl}_{3}\right): \delta=188.5,161.0,153.2,150.8,143.8,143.7,123.8,122.9,120.5,37.2,33.9,30.3,0.2$. HRMS (ESI-TOF) m/z: $[\mathrm{M}+\mathrm{H}]^{+}$Calcd for $\mathrm{C}_{17} \mathrm{H}_{25} \mathrm{O}_{2} \mathrm{Si}$ 289.1618; Found 289.1615. FTIR (KBr, $\left.\mathrm{cm}^{-1}\right): 3444.53,3417.81,3174.82,1659.95,1651.53,1455.12,1398.33,840.03$.

(3-Methoxy-3-((trimethylsilyl)oxy)-1H-indene-2-carbaldehyde (3ha)

Following the general procedure, 3ha was obtained as a pale yellow solid by the purification with flash column chromatography on silica gel $(\mathrm{PE}: \mathrm{EA}=70: 1)$, m.p.: $121.8-123.1{ }^{\circ} \mathrm{C}, 26.7 \mathrm{mg}$, yield $=$ 51\%. ${ }^{1} \mathrm{H}$ NMR (500 MHz, $\left.\mathrm{CDCl}_{3}\right): \delta=10.17(\mathrm{~s}, 1 \mathrm{H}), 7.64(\mathrm{~d}, J=9.0 \mathrm{~Hz}, 1 \mathrm{H}), 7.10(\mathrm{~d}, J=2.0 \mathrm{~Hz}$, 1H), $6.91(\mathrm{dd}, J=8.5 \mathrm{~Hz}, J=2.5 \mathrm{~Hz}, 1 \mathrm{H}), 3.86(\mathrm{~s}, 3 \mathrm{H}), 3.69(\mathrm{~s}, 2 \mathrm{H}), 0.49(\mathrm{~s}, 9 \mathrm{H}) .{ }^{13} \mathrm{C}$ NMR $(125$ $\left.\mathrm{Hz}, \mathrm{CDCl}_{3}\right): \delta=188.0,161.2,159.3,151.8,146.2,139.4,125.1,112.3,108.6,54.4,37.2,0.2$. HRMS (ESI-TOF) m/z: $[\mathrm{M}+\mathrm{H}]^{+}$Calcd for $\mathrm{C}_{14} \mathrm{H}_{19} \mathrm{O}_{3} \mathrm{Si}$ 263.1098; Found 263.1100. FTIR (KBr, $\left.\mathrm{cm}^{-1}\right): 3584.82,3472.65,3444.32,3175.42,1659.84,1644.79,1402.44,1182.43$.<smiles>COC1=C(C=O)Cc2cc(OC(F)(F)F)ccc21</smiles>

6-(Trifluoromethoxy)-3-((trimethylsilyl)oxy)-1H-indene-2-carbaldeh yde (3ia)

Following the general procedure, 3ia was obtained as a white solid by the purification with flash column chromatography on silica gel $(\mathrm{PE}: \mathrm{EA}=160: 1)$, m.p.: $75.6-76.9^{\circ} \mathrm{C}, 39.2 \mathrm{mg}$, yield $=62 \%$. ${ }^{1} \mathrm{H} \mathrm{NMR}\left(500 \mathrm{MHz}, \mathrm{CDCl}_{3}\right): \delta=10.24(\mathrm{~s}, 1 \mathrm{H}), 7.74(\mathrm{~d}, J=11.0 \mathrm{~Hz}, 1 \mathrm{H}), 7.41(\mathrm{~s}, 1 \mathrm{H}), 7.22(\mathrm{dd}, J$ $=8.5 \mathrm{~Hz}, J=1.0 \mathrm{~Hz}, 1 \mathrm{H}), 3.75(\mathrm{~s}, 2 \mathrm{H}), 0.51(\mathrm{~s}, 9 \mathrm{H}) .{ }^{13} \mathrm{C} \mathrm{NMR}\left(125 \mathrm{~Hz}, \mathrm{CDCl}_{3}\right): \delta=188.3,159.5$, $154.5,148.2\left(\mathrm{~d}, J_{\mathrm{C}-\mathrm{F}}=1.6 \mathrm{~Hz}\right), 145.6,145.0,125.1,119.5\left(\mathrm{q}, J_{\mathrm{C}-\mathrm{F}}=255.9 \mathrm{~Hz}\right), 118.6,116.3,37.7$, 0.2. HRMS (ESI-TOF) m/z: $[\mathrm{M}+\mathrm{H}]^{+}$Calcd for $\mathrm{C}_{14} \mathrm{H}_{16} \mathrm{~F}_{3} \mathrm{O}_{3} \mathrm{Si}$ 317.0815; Found 317.0820. FTIR $\left(\mathrm{KBr}, \mathrm{cm}^{-1}\right): 3473.08,3416.85,3384.55,3225.49,1659.96,1402.54,1254.56,1158.41,840.66$. 


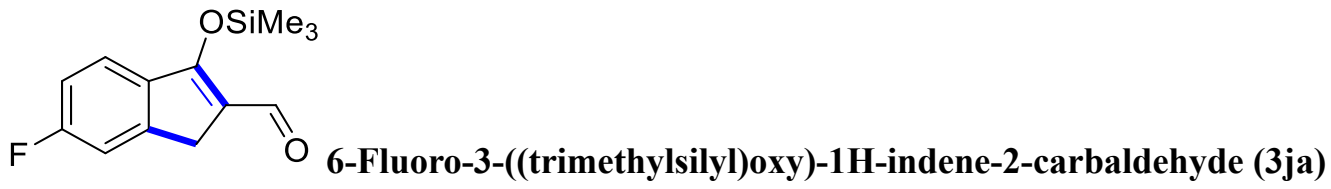

Following the general procedure, $\mathbf{3 j a}$ was obtained as a white solid by the purification with flash column chromatography on silica gel $(\mathrm{PE}: \mathrm{EA}=160: 1)$, m.p.: $98.4-105.1^{\circ} \mathrm{C}, 35.5 \mathrm{mg}$, yield $=71 \%$. ${ }^{1} \mathrm{H}$ NMR $\left(500 \mathrm{MHz}, \mathrm{CDCl}_{3}\right): \delta=10.21(\mathrm{~s}, 1 \mathrm{H}), 7.68(\mathrm{dd}, J=8.5 \mathrm{~Hz}, J=5.0 \mathrm{~Hz}, 1 \mathrm{H}), 7.25(\mathrm{~d}, J=$ $8.5 \mathrm{~Hz}, 1 \mathrm{H}), 7.06(\mathrm{td}, J=8.5 \mathrm{~Hz}, J=2.0 \mathrm{~Hz}, 1 \mathrm{H}), 3.71(\mathrm{~s}, 2 \mathrm{H}), 0.50(\mathrm{~s}, 9 \mathrm{H}) .{ }^{13} \mathrm{C}$ NMR $(125$ $\left.\mathrm{Hz}, \mathrm{CDCl}_{3}\right): \delta=188.1,162.1\left(\mathrm{~d}, J_{\mathrm{C}-\mathrm{F}}=247.4 \mathrm{~Hz}\right), 160.0,153.5\left(\mathrm{~d}, J_{\mathrm{C}-\mathrm{F}}=3.9 \mathrm{~Hz}\right), 146.2\left(\mathrm{~d}, J_{\mathrm{C}-\mathrm{F}}=\right.$ $9.1 \mathrm{~Hz}), 142.4\left(\mathrm{~d}, J_{\mathrm{C}-\mathrm{F}}=2.3 \mathrm{~Hz}\right), 125.3\left(\mathrm{~d}, J_{\mathrm{C}-\mathrm{F}}=9.1 \mathrm{~Hz}\right), 113.1\left(\mathrm{~d}, J_{\mathrm{C}-\mathrm{F}}=23 \mathrm{~Hz}\right), 110.9\left(\mathrm{~d}, J_{\mathrm{C}-\mathrm{F}}=\right.$ $22.8 \mathrm{~Hz}), 37.5\left(\mathrm{~d}, J_{\mathrm{C}-\mathrm{F}}=2.5 \mathrm{~Hz}\right), 0.2$. HRMS (ESI-TOF) m/z: $[\mathrm{M}+\mathrm{H}]^{+}$Calcd for $\mathrm{C}_{13} \mathrm{H}_{16} \mathrm{FO}_{2} \mathrm{Si}$ 251.0898; Found 251.0900. FTIR (KBr, $\left.\mathrm{cm}^{-1}\right)$ : 3472.65, 3444.32, 3175.42, 1659.84, 1651.59, $1402.44,1182.43,841.78$.

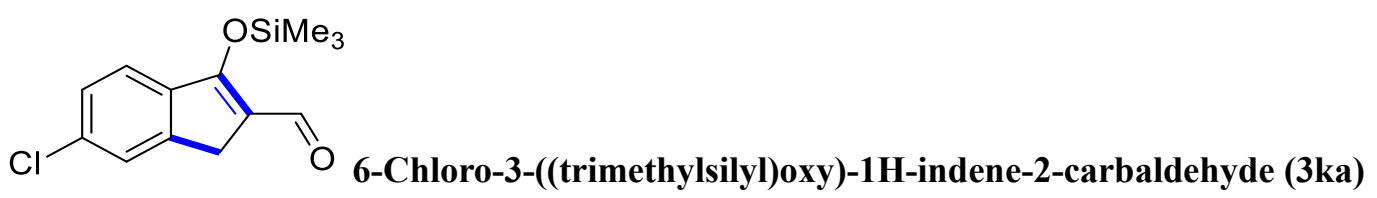

Following the general procedure, 3ka was obtained as a pale yellow solid by the purification with flash column chromatography on silica gel $(\mathrm{PE}: \mathrm{EA}=160: 1)$, m. p.: $92.7-93.2{ }^{\circ} \mathrm{C}, 30.9 \mathrm{mg}$, yield $=$ 58\%. ${ }^{1} \mathrm{H}$ NMR (500 MHz, $\left.\mathrm{CDCl}_{3}\right): \delta=10.23(\mathrm{~s}, 1 \mathrm{H}), 7.64(\mathrm{~d}, J=8.0 \mathrm{~Hz}, 1 \mathrm{H}), 7.53(\mathrm{~s}, 1 \mathrm{H}), 7.33$ $(\mathrm{dd}, J=8.5 \mathrm{~Hz}, J=2.0 \mathrm{~Hz}, 1 \mathrm{H}), 3.70(\mathrm{~s}, 2 \mathrm{H}), 0.50(\mathrm{~s}, 9 \mathrm{H}) .{ }^{13} \mathrm{C} \mathrm{NMR}\left(125 \mathrm{~Hz}, \mathrm{CDCl}_{3}\right): \delta=188.4$, $159.8,153.7,145.3,144.9,133.4,126.1,125.0,123.9,37.3,0.2$. HRMS (ESI-TOF) m/z: $[\mathrm{M}+\mathrm{H}]^{+}$ Calcd for $\mathrm{C}_{13} \mathrm{H}_{16} \mathrm{ClO}_{2} \mathrm{Si} 267.0603$; Found 267.0600. FTIR (KBr, $\left.\mathrm{cm}^{-1}\right)$ : 3443.88, 3417.49, 3175.63, $1660.84,1402.39,1241.12,845.57,818.75$.

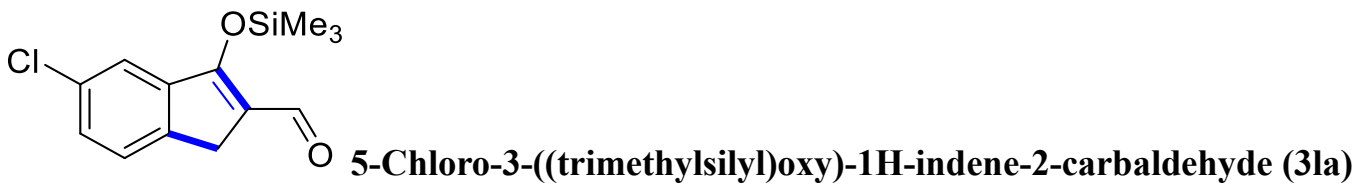

Following the general procedure, 3la was obtained as a yellow oil by the purification with flash column chromatography on silica gel $(\mathrm{PE}: \mathrm{EA}=140: 1), 14.9 \mathrm{mg}$, yield $=28 \% .{ }^{1} \mathrm{H} \mathrm{NMR}(500 \mathrm{MHz}$, $\left.\mathrm{CDCl}_{3}\right): \delta=10.25(\mathrm{~s}, 1 \mathrm{H}), 7.68(\mathrm{~d}, J=1.5 \mathrm{~Hz}, 1 \mathrm{H}), 7.47(\mathrm{~d}, J=8.0 \mathrm{~Hz}, 1 \mathrm{H}), 7.33(\mathrm{dd}, J=8.0 \mathrm{~Hz}$, 
$J=1.5 \mathrm{~Hz}, 1 \mathrm{H}), 3.69(\mathrm{~s}, 2 \mathrm{H}), 0.51(\mathrm{~s}, 9 \mathrm{H}) .{ }^{13} \mathrm{C} \mathrm{NMR}\left(125 \mathrm{~Hz}, \mathrm{CDCl}_{3}\right): \delta=188.5,159.6,154.9$, 148.0, 147.7, 131.6, 127.0, 124.4, 124.2, 37.1, 0.2. HRMS (ESI-TOF) $\mathrm{m} / \mathrm{z}:[\mathrm{M}+\mathrm{H}]^{+}$Calcd for $\mathrm{C}_{13} \mathrm{H}_{16} \mathrm{ClO}_{2} \mathrm{Si}$ 267.0603; Found 267.0603. FTIR $\left(\mathrm{KBr}, \mathrm{cm}^{-1}\right): 3452.07,3423.13,3385.24,2922.98$, $2359.82,1651.58,1399.27,841.79$.<smiles>COC1=C(C=O)Cc2c(Cl)cccc21</smiles>

7-Chloro-3-((trimethylsilyl)oxy)-1H-indene-2-carbaldehyde (3la')

Following the general procedure, 3la' was obtained as a yellow gum by the purification with flash column chromatography on silica gel $(\mathrm{PE}: \mathrm{EA}=200: 1), 9.0 \mathrm{mg}$, yield $=17 \% .{ }^{1} \mathrm{H} \mathrm{NMR}(500 \mathrm{MHz}$, $\left.\mathrm{CDCl}_{3}\right): \delta=10.26(\mathrm{~s}, 1 \mathrm{H}), 7.64(\mathrm{dd}, J=7.5 \mathrm{~Hz}, J=1.5 \mathrm{~Hz}, 1 \mathrm{H}), 7.35-7.30(\mathrm{~m}, 2 \mathrm{H}), 3.75(\mathrm{~s}, 2 \mathrm{H})$, 0.51 (s, 9H). ${ }^{13} \mathrm{C}$ NMR $\left(125 \mathrm{~Hz}, \mathrm{CDCl}_{3}\right): \delta=188.4,160.1,153.7,147.8,141.5,129.7,127.3,127.1$, 122.7, 37.4, 0.2. HRMS (ESI-TOF) m/z: $[\mathrm{M}+\mathrm{H}]^{+}$Calcd for $\mathrm{C}_{13} \mathrm{H}_{16} \mathrm{ClO}_{2} \mathrm{Si}$ 267.0603; Found 267.0598. FTIR (KBr, $\left.\mathrm{cm}^{-1}\right)$ : 3474.76, 3449.87, 3424.16, 2985.53, 2956.17, 2354.39, 1644.84, $1633.64,1402.65$.

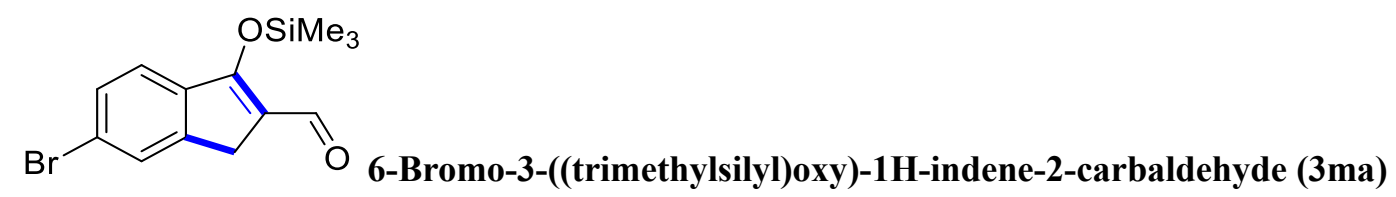

Following the general procedure, $\mathbf{3 m a}$ was obtained as a yellow solid by the purification with flash column chromatography on silica gel (PE:EA $=160: 1)$, m.p.: $118.1-121.0^{\circ} \mathrm{C}, 44.0 \mathrm{mg}$, yield $=71 \%$. ${ }^{1} \mathrm{H}$ NMR (500 MHz, $\left.\mathrm{CDCl}_{3}\right): \delta=10.24(\mathrm{~s}, 1 \mathrm{H}), 7.68(\mathrm{~s}, 1 \mathrm{H}), 7.58(\mathrm{~d}, J=8.5 \mathrm{~Hz}, 1 \mathrm{H}), 7.47(\mathrm{~d}, J=$ $8.0 \mathrm{~Hz}, 1 \mathrm{H}), 3.69(\mathrm{~s}, 2 \mathrm{H}), 0.50(\mathrm{~s}, 9 \mathrm{H}) .{ }^{13} \mathrm{C} \mathrm{NMR}\left(125 \mathrm{~Hz}, \mathrm{CDCl}_{3}\right): \delta=188.4,159.8,153.6,145.5$, 145.3, 128.9, 126.9, 125.4, 121.7, 37.3, 0.2. HRMS (ESI-TOF) $\mathrm{m} / \mathrm{z}:[\mathrm{M}+\mathrm{H}]^{+}$Calcd for $\mathrm{C}_{13} \mathrm{H}_{16} \mathrm{BrO}_{2} \mathrm{Si}$ 311.0097; Found 311.0096. FTIR (KBr, cm$\left.{ }^{-1}\right): 3452.10,3417.62,3209.15,1657.27$, $1652.02,1402.74,844.84,817.34$.<smiles>COC1=C(C=O)Cc2cc(-c3ccccc3)ccc21</smiles>

6-Phenyl-3-((trimethylsilyl)oxy)-1H-indene-2-carbaldehyde (3na) 
Following the general procedure, 3na was obtained as a yellow solid by the purification with flash column chromatography on silica gel (PE:EA $=100: 1)$, m.p.: $138.5-140.7{ }^{\circ} \mathrm{C}, 43.8 \mathrm{mg}$, yield $=71 \%$. ${ }^{1} \mathrm{H} \mathrm{NMR}\left(500 \mathrm{MHz}, \mathrm{CDCl}_{3}\right): \delta=10.28(\mathrm{~s}, 1 \mathrm{H}), 7.83(\mathrm{~d}, J=8.0 \mathrm{~Hz}, 1 \mathrm{H}), 7.80(\mathrm{~s}, 1 \mathrm{H}), 7.65(\mathrm{~d}, J=$ $7.0 \mathrm{~Hz}, 2 \mathrm{H}), 7.62(\mathrm{~d}, J=8.0 \mathrm{~Hz}, 1 \mathrm{H}), 7.47(\mathrm{t}, J=7.5 \mathrm{~Hz}, 2 \mathrm{H}), 7.38(\mathrm{t}, \mathrm{J}=7.0 \mathrm{~Hz}, 1 \mathrm{H}), 3.80(\mathrm{~s}, 2 \mathrm{H})$, 0.55 (s, 9H). ${ }^{13} \mathrm{C}$ NMR $\left(125 \mathrm{~Hz}, \mathrm{CDCl}_{3}\right): \delta=188.4,160.6,153.7,145.5,144.3,140.2,139.7,127,7$, 126.5, 126.1, 124.8, 124.5, 122.2, 37.3, 0.2. HRMS (ESI-TOF) m/z: $[\mathrm{M}+\mathrm{H}]^{+}$Calcd for $\mathrm{C}_{19} \mathrm{H}_{21} \mathrm{O}_{2} \mathrm{Si}$ 309.1305; Found 309.1302. FTIR (KBr, $\left.\mathrm{cm}^{-1}\right)$ : 3472.61, 3444.42, 3175.51, 1651.32, 1645.16, $1402.35,1247.68,849.32,769.88$.<smiles>COC1=C(C=O)Cc2cc3ccccc3cc21</smiles>

3-((Trimethylsilyl)oxy)-1H-cyclopenta[b]naphthalene-2-carbaldehyde (3oa)

Following the general procedure, 3oa was obtained as a yellow solid by the purification with flash column chromatography on silica gel (PE:EA $=160: 1)$, m.p.: $141.9-143.0^{\circ} \mathrm{C}, 36.1 \mathrm{mg}$, yield $=64 \%$. ${ }^{1} \mathrm{H} \mathrm{NMR}\left(500 \mathrm{MHz}, \mathrm{CDCl}_{3}\right): \delta=10.32(\mathrm{~s}, 1 \mathrm{H}), 8.17(\mathrm{~s}, 1 \mathrm{H}), 7.91(\mathrm{~d}, J=8 \mathrm{~Hz}, 2 \mathrm{H}), 7.83(\mathrm{~d}, J=7.5$ $\mathrm{Hz}, 1 \mathrm{H}), 7.51-7.45(\mathrm{~m}, 2 \mathrm{H}), 3.82(\mathrm{~s}, 2 \mathrm{H}), 0.58(\mathrm{t}, J=1.0 \mathrm{~Hz}, 9 \mathrm{H}) .{ }^{13} \mathrm{C} \mathrm{NMR}\left(125 \mathrm{~Hz}, \mathrm{CDCl}_{3}\right): \delta=$ 188.8, 160.1, 154.2, 145.2, 139.9, 132.1, 131.3, 127.5, 126.4, 125.3, 124.2, 123.5, 121.6, 36.1, 0.2. HRMS (ESI-TOF) m/z: $[\mathrm{M}+\mathrm{H}]^{+}$Calcd for $\mathrm{C}_{17} \mathrm{H}_{19} \mathrm{O}_{2} \mathrm{Si}$ 283.1149; Found 283.1153. FTIR (KBr, $\left.\mathrm{cm}^{-1}\right): 3472.65,3444.32,3175.42,1651.59,1644.79,1402.44,1182.43,841.78$.<smiles>COC1=C(C=O)Cc2c(C)cc(C)cc21</smiles>

5,7-Dimethyl-3-((trimethylsilyl)oxy)-1H-indene-2-carbaldehyde (3pa)

Following the general procedure, 3pa was obtained as a yellow solid by the purification with flash column chromatography on silica gel (PE:EA $=160: 1)$, m.p.: $106.0-107.8^{\circ} \mathrm{C}, 13.0 \mathrm{mg}$, yield $=25 \%$. ${ }^{1} \mathrm{H} \mathrm{NMR}\left(500 \mathrm{MHz}, \mathrm{CDCl}_{3}\right): \delta=10.24$ (s, 1H), 7.39 (s, 1H), 7.02 (s, 1H), 3.57 (s, 2H), $2.40(\mathrm{~s}, 3 \mathrm{H})$, $2.34(\mathrm{~s}, 3 \mathrm{H}), 0.50(\mathrm{~s}, 9 \mathrm{H}) .{ }^{13} \mathrm{C} \mathrm{NMR}\left(125 \mathrm{~Hz}, \mathrm{CDCl}_{3}\right): \delta=188.6,161.3,153.1,146.2,139.5,135.5$, 132.4, 129.1, 122.3, 35.7, 20.4, 17.6, 0.2. HRMS (ESI-TOF) m/z: $[\mathrm{M}+\mathrm{H}]^{+}$Calcd for $\mathrm{C}_{15} \mathrm{H}_{21} \mathrm{O}_{2} \mathrm{Si}$ 261.1305; Found 261.1306. FTIR (KBr, $\left.\mathrm{cm}^{-1}\right)$ : 3444.57, 3417.72, 3175.22, 1651.17, 1645.31, 


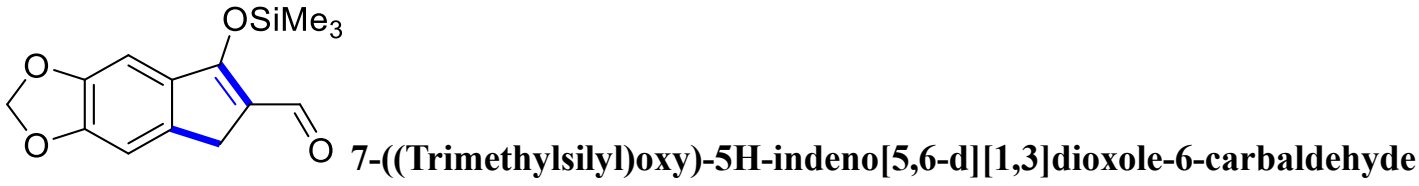

(3qa)

Following the general procedure, 3qa was obtained as a white solid by the purification with flash column chromatography on silica gel (PE:EA $=50: 1)$, m.p.: $158.6-161.1{ }^{\circ} \mathrm{C}, 33.7 \mathrm{mg}$, yield $=61 \%$. ${ }^{1} \mathrm{H} \mathrm{NMR}\left(500 \mathrm{MHz}, \mathrm{CDCl}_{3}\right): \delta=10.19(\mathrm{~s}, 1 \mathrm{H}), 7.27(\mathrm{~d}, J=8.0 \mathrm{~Hz}, 1 \mathrm{H}), 6.86(\mathrm{~d}, J=8.0 \mathrm{~Hz}, 1 \mathrm{H})$, $6.03(\mathrm{~s}, 2 \mathrm{H}), 3.66(\mathrm{~s}, 2 \mathrm{H}), 0.49$ (s, 9H). ${ }^{13} \mathrm{C}$ NMR $\left(125 \mathrm{~Hz}, \mathrm{CDCl}_{3}\right): \delta=188.1,160.7,151.8,147.0$, 142.6, 142.0, 122.8, 118.3, 106.1, 100.3, 33.3, 0.2. HRMS (ESI-TOF) m/z: $[\mathrm{M}+\mathrm{H}]^{+}$Calcd for $\mathrm{C}_{14} \mathrm{H}_{17} \mathrm{O}_{4} \mathrm{Si}$ 277.0891; Found 277.0887. FTIR (KBr, $\left.\mathrm{cm}^{-1}\right)$ : 3444.53, 3417.70, 3173.08, 3144.11, $1644.42,1470.81,1402.09,842.75,804.84$.<smiles>COC(=O)c1cc2c(cc1/C=C/C=O)OCO2</smiles>

(E)-3-(6-((trimethylsilyl)carbonyl)benzo[d][1,3]dioxol-5-yl)acrylaldeh yde (5qa)

Following the general procedure, 5qa was obtained as a yellow solid by the purification with flash column chromatography on silica gel $(\mathrm{PE}: \mathrm{EA}=15: 1)$, m.p.: $145.9-147.8^{\circ} \mathrm{C}, 7.2 \mathrm{mg}$, yield $=13 \%$. ${ }^{1} \mathrm{H}$ NMR $\left(500 \mathrm{MHz}, \mathrm{CDCl}_{3}\right): \delta=9.70(\mathrm{~d}, J=8.0 \mathrm{~Hz}, 1 \mathrm{H}), 7.95(\mathrm{~d}, J=16.0 \mathrm{~Hz}, 1 \mathrm{H}), 7.39(\mathrm{~d}, J=$ $8.0 \mathrm{~Hz}, 1 \mathrm{H}), 6.95(\mathrm{q}, J=8.0 \mathrm{~Hz}, 2 \mathrm{H}), 6.15(\mathrm{~s}, 2 \mathrm{H}), 0.36(\mathrm{~s}, 9 \mathrm{H}) .{ }^{13} \mathrm{C} \mathrm{NMR}\left(125 \mathrm{~Hz}, \mathrm{CDCl}_{3}\right): \delta=$ 239.4, 196.7, 151.9, 149.4, 147.3, 137.7, 135.3, 129.2, 116.3, 110.0, 103.8, 0.2. HRMS (ESI-TOF) m/z: $[\mathrm{M}+\mathrm{H}]^{+}$Calcd for $\mathrm{C}_{14} \mathrm{H}_{17} \mathrm{O}_{4}$ Si 277.0891; Found 277.0894. FTIR $\left(\mathrm{KBr}, \mathrm{cm}^{-1}\right)$ : 3453.49, $3415.89,2920.89,2357.63,2330.02,1632.90,1402.66,1013.76$.<smiles>COC1=C(C=O)Cc2cc(OC)c(F)cc21</smiles>
de (3ra) 
Following the general procedure, 3ra was obtained as a white solid by the purification with flash column chromatography on silica gel $(\mathrm{PE}: \mathrm{EA}=80: 1)$, m.p.: $173.4-173.8^{\circ} \mathrm{C}, 41.5 \mathrm{mg}$, yield $=74 \%$. ${ }^{1} \mathrm{H}$ NMR $\left(500 \mathrm{MHz}, \mathrm{CDCl}_{3}\right): \delta=10.18(\mathrm{~s}, 1 \mathrm{H}), 7.45(\mathrm{~d}, J=8.5 \mathrm{~Hz}, 1 \mathrm{H}), 6.98(\mathrm{t}, J=8.0 \mathrm{~Hz}, 1 \mathrm{H})$, $3.94(\mathrm{~s}, 3 \mathrm{H}), 3.72(\mathrm{~s}, 2 \mathrm{H}), 1.25$ (s, 3H), 0.49 (s, 9H). ${ }^{13} \mathrm{C} \mathrm{NMR}\left(125 \mathrm{~Hz}, \mathrm{CDCl}_{3}\right): \delta=187.9,160.3$, $152.4\left(\mathrm{~d}, J_{\mathrm{C}-\mathrm{F}}=1.0 \mathrm{~Hz}\right), 147.6\left(\mathrm{~d}, J_{\mathrm{C}-\mathrm{F}}=247.4 \mathrm{~Hz}\right), 147.1\left(\mathrm{~d}, J_{\mathrm{C}-\mathrm{F}}=10.4 \mathrm{~Hz}\right), 141.3\left(\mathrm{~d}, J_{\mathrm{C}-\mathrm{F}}=4.0\right.$ $\mathrm{Hz}), 130.3\left(\mathrm{~d}, J_{\mathrm{C}-\mathrm{F}}=14.3 \mathrm{~Hz}\right), 120.2\left(\mathrm{~d}, J_{\mathrm{C}-\mathrm{F}}=3.6 \mathrm{~Hz}\right), 111.3,55.5,33.9,0.2$. HRMS (ESI-TOF) m/z: $[\mathrm{M}+\mathrm{H}]^{+}$Calcd for $\mathrm{C}_{14} \mathrm{H}_{18} \mathrm{FO}_{3} \mathrm{Si}$ 281.1004; Found 281.1009. FTIR $\left(\mathrm{KBr}, \mathrm{cm}^{-1}\right)$ : 3416.48, $1682.17,1634.67,1620.57,803.56,618.45,480.91$.

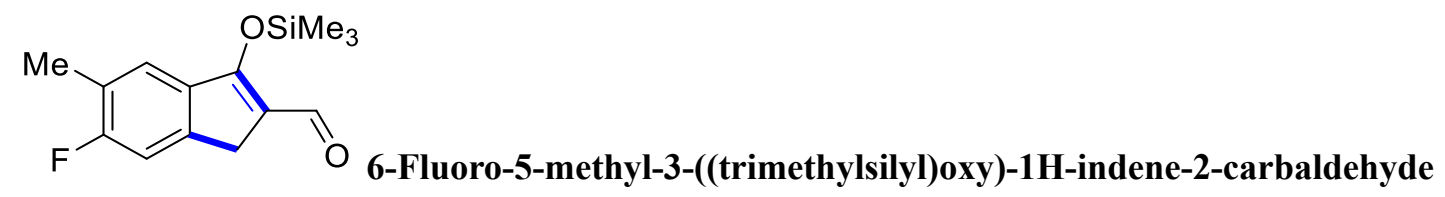

(3sa)

Following the general procedure, 3sa was obtained as a yellow solid by the purification with flash column chromatography on silica gel (PE:EA $=100: 1)$, m.p.: $105.1-105.7{ }^{\circ} \mathrm{C}, 37.5 \mathrm{mg}$, yield $=71 \%$. ${ }^{1} \mathrm{H} \mathrm{NMR}\left(500 \mathrm{MHz}, \mathrm{CDCl}_{3}\right): \delta=10.19(\mathrm{~s}, 1 \mathrm{H}), 7.52(\mathrm{~d}, J=7.0 \mathrm{~Hz}, 1 \mathrm{H}), 7.19(\mathrm{~d}, J=7.19 \mathrm{~Hz}, 1 \mathrm{H})$, $3.67(\mathrm{~s}, 2 \mathrm{H}), 2.34(\mathrm{~s}, 3 \mathrm{H}), 0.51(\mathrm{~d}, J=1.0 \mathrm{~Hz}, 9 \mathrm{H}) .{ }^{13} \mathrm{C} \mathrm{NMR}\left(125 \mathrm{~Hz}, \mathrm{CDCl}_{3}\right): \delta=188.1,160.58$ $\left(\mathrm{d}, J_{\mathrm{C}-\mathrm{F}}=246.5 \mathrm{~Hz}\right), 160.2,153.5\left(\mathrm{~d}, J_{\mathrm{C}-\mathrm{F}}=3.9 \mathrm{~Hz}\right), 143.4\left(\mathrm{~d}, J_{\mathrm{C}-\mathrm{F}}=11.3 \mathrm{~Hz}\right), 142.2\left(\mathrm{~d}, J_{\mathrm{C}-\mathrm{F}}=2.5\right.$ $\mathrm{Hz}), 126.6\left(\mathrm{~d}, J_{\mathrm{C}-\mathrm{F}}=6.3 \mathrm{~Hz}\right), 122.4\left(\mathrm{~d}, J_{\mathrm{C}-\mathrm{F}}=18.8 \mathrm{~Hz}\right), 110.3\left(\mathrm{~d}, J_{\mathrm{C}-\mathrm{F}}=23.8 \mathrm{~Hz}\right), 37.2\left(\mathrm{~d}, J_{\mathrm{C}-\mathrm{F}}=2.5\right.$ $\mathrm{Hz}), 13.9\left(\mathrm{~d}, J_{\mathrm{C}-\mathrm{F}}=3.8 \mathrm{~Hz}\right.$ ), 0.2. HRMS (ESI-TOF) $\mathrm{m} / \mathrm{z}:[\mathrm{M}+\mathrm{H}]^{+} \mathrm{Calcd}$ for $\mathrm{C}_{14} \mathrm{H}_{18} \mathrm{FO}_{2} \mathrm{Si}$ 265.1055; Found 265.1059. FTIR (KBr, cm-1): 3416.49, 1651.53, 1634.53, 1615.59, 838.39, 618.38, 469.84.<smiles>CC(=O)c1cc(C)c(F)cc1/C=C/C=O</smiles>

(E)-3-(5-fluoro-4-methyl-2-((trimethylsilyl)carbonyl)phenyl)acrylald ehyde (5sa)

Following the general procedure, 5sa was obtained as a yellow solid by the purification with flash column chromatography on silica gel (PE:EA $=60: 1)$, m.p.: 88.3-89. ${ }^{\circ} \mathrm{C}, 3.2 \mathrm{mg}$, yield $=6 \% .{ }^{1} \mathrm{H}$ NMR (500 MHz, $\left.\mathrm{CDCl}_{3}\right): \delta=9.72(\mathrm{~d}, J=7.5 \mathrm{~Hz}, 1 \mathrm{H}), 7.90(\mathrm{~d}, J=16.0 \mathrm{~Hz}, 1 \mathrm{H}), 7.60(\mathrm{~d}, J=7.0$ $\mathrm{Hz}, 1 \mathrm{H}), 6.52(\mathrm{q}, J=8.0 \mathrm{~Hz}, 1 \mathrm{H}), 2.39(\mathrm{~s}, 3 \mathrm{H}), 0.37(\mathrm{~s}, 9 \mathrm{H}) .{ }^{13} \mathrm{C} \mathrm{NMR}\left(125 \mathrm{~Hz}, \mathrm{CDCl}_{3}\right): \delta=241.2$, 
$195.5,164.2\left(\mathrm{~d}, J_{\mathrm{C}-\mathrm{F}}=251.4 \mathrm{~Hz}\right), 152.1\left(\mathrm{~d}, J_{\mathrm{C}-\mathrm{F}}=1.8 \mathrm{~Hz}\right), 140.0\left(\mathrm{~d}, J_{\mathrm{C}-\mathrm{F}}=3.4 \mathrm{~Hz}\right), 135.9\left(\mathrm{~d}, J_{\mathrm{C}-\mathrm{F}}=\right.$ $6.6 \mathrm{~Hz}), 134.2\left(\mathrm{~d}, J_{\mathrm{C}-\mathrm{F}}=8.3 \mathrm{~Hz}\right), 132.6,129.1\left(\mathrm{~d}, J_{\mathrm{C}-\mathrm{F}}=17.9 \mathrm{~Hz}\right), 116.6\left(\mathrm{~d}, J_{\mathrm{C}-\mathrm{F}}=23.6 \mathrm{~Hz}\right), 16.5(\mathrm{~d}$, $J_{\mathrm{C}-\mathrm{F}}=2.9 \mathrm{~Hz}$ ), 0.2. HRMS (ESI-TOF) $\mathrm{m} / \mathrm{z}:[\mathrm{M}+\mathrm{H}]^{+}$Calcd for $\mathrm{C}_{14} \mathrm{H}_{18} \mathrm{FO}_{2} \mathrm{Si}$ 265.1055; Found 265.1057. FTIR (KBr, $\left.\mathrm{cm}^{-1}\right)$ : 3441.64, 3418.32, 2900.10, 2359.36, 2329.81, 1682.78, 1402.63, 845.11.

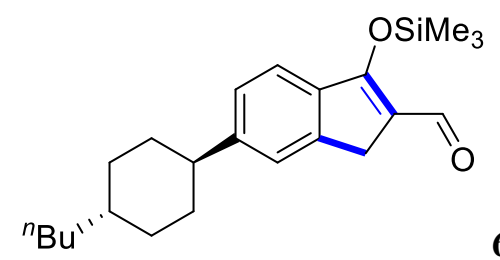

6-((1s,4r)-4-Butylcyclohexyl)-3-((trimethylsilyl)oxy)-1H-inden

e-2-carbaldehyde (3ta)

Following the general procedure, 3ta was obtained as a yellow solid by the purification with flash column chromatography on silica gel $(\mathrm{PE}: \mathrm{EA}=160: 1)$, m.p.: $49.6-55.2^{\circ} \mathrm{C}, 51.1 \mathrm{mg}$, yield $=69 \%$. ${ }^{1} \mathrm{H}$ NMR $\left(500 \mathrm{MHz}, \mathrm{CDCl}_{3}\right): \delta=10.22(\mathrm{~d}, J=2.0 \mathrm{~Hz}, 1 \mathrm{H}), 7.66(\mathrm{dd}, J=8.0 \mathrm{~Hz}, J=1.5 \mathrm{~Hz}, 1 \mathrm{H})$, $7.42(\mathrm{~s}, 1 \mathrm{H}), 7.21(\mathrm{~d}, J=8.0 \mathrm{~Hz}, 1 \mathrm{H}), 3.69(\mathrm{~s}, 2 \mathrm{H}), 2.53(\mathrm{t}, J=12.0 \mathrm{~Hz}, 1 \mathrm{H}), 1.90(\mathrm{t}, \mathrm{J}=10.5 \mathrm{~Hz}$, 4H), 1.53-1.45 (m, 2H), $1.31(\mathrm{~d}, J=3.0 \mathrm{~Hz}, 4 \mathrm{H}), 1.25(\mathrm{~d}, J=6.5 \mathrm{~Hz}, 3 \mathrm{H}), 1.10-1.03(\mathrm{~m}, 2 \mathrm{H}), 0.92-$ $0.91(\mathrm{~m}, 3 \mathrm{H}), 0.49(\mathrm{~d}, J=2.0 \mathrm{~Hz}, 9 \mathrm{H}) .{ }^{13} \mathrm{C} \mathrm{NMR}\left(125 \mathrm{~Hz}, \mathrm{CDCl}_{3}\right): \delta=188.4,161.1,153.0,147.6$, 144.2, 144.0, 124.5, 124.1, 121.9, 43.7, 37.1, 36.1, 35.9, 33.3, 32.4, 28.1, 21.9, 13.0, 0.2. HRMS (ESI-TOF) m/z: $[\mathrm{M}+\mathrm{H}]^{+}$Calcd for $\mathrm{C}_{23} \mathrm{H}_{35} \mathrm{O}_{2} \mathrm{Si}$ 371.2401; Found 371.2404. FTIR $\left(\mathrm{KBr}, \mathrm{cm}^{-1}\right)$ : $3452.48,3417.55,2955.86,2921.81,2851.66,1659.70,1393.30,840.93$.<smiles>COC1=C(C)Cc2ccccc21</smiles>

\section{Trimethyl((2-methyl-1H-inden-3-yl)oxy)silane (4ab)}

Following the general procedure, $4 \mathbf{a b}$ was obtained as a brown oil by the purification with flash column chromatography on silica gel using PE as the eluent, $31.0 \mathrm{mg}$, yield $=71 \%$. ${ }^{1} \mathrm{H}$ NMR $(500$ $\left.\mathrm{MHz}, \mathrm{CDCl}_{3}\right): \delta=7.42-7.37(\mathrm{dd}, J=17.0 \mathrm{~Hz}, J=7.5 \mathrm{~Hz}, 2 \mathrm{H}), 7.21(\mathrm{t}, J=7.5 \mathrm{~Hz}, 1 \mathrm{H}), 7.08(\mathrm{t}, J=$ $7.0 \mathrm{~Hz}, 1 \mathrm{H}), 3.36$ (s, 2H), 2.22 (s, 3H), 0.35 (s, 9H). ${ }^{13} \mathrm{C} \mathrm{NMR}\left(125 \mathrm{~Hz}, \mathrm{CDCl}_{3}\right): \delta=155.4,149.7$, 142.8, 135.2, 125.6, 122.8, 122.6, 120.8, 46.0, 17.1, 0.2. HRMS (ESI-TOF) m/z: $[\mathrm{M}+\mathrm{H}]^{+}$Calcd for $\mathrm{C}_{13} \mathrm{H}_{19} \mathrm{OSi}$ 219.1200; Found 219.1195. FTIR ( $\left.\mathrm{KBr}, \mathrm{cm}^{-1}\right)$ : 3473.09, 3453.12, 3225.51, 1651.59, 
$1644.80,1633.88,1402.39,1385.06$.

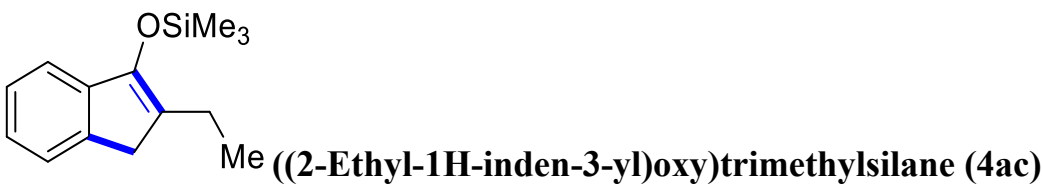

Following the general procedure, 4 ac was obtained as a yellow oil by the purification with flash column chromatography on silica gel using PE as the eluent, $24.0 \mathrm{mg}$, yield $=52 \%$. ${ }^{1} \mathrm{H}$ NMR $(500$ $\left.\mathrm{MHz}, \mathrm{CDCl}_{3}\right): \delta=7.44-7.39(\mathrm{dd}, J=19.0 \mathrm{~Hz}, J=7.5 \mathrm{~Hz}, 2 \mathrm{H}), 7.23-7.20(\mathrm{~m}, 1 \mathrm{H}), 7.10-7.07(\mathrm{~m}$, 1H), $3.40(\mathrm{~s}, 2 \mathrm{H}), 2.61(\mathrm{q}, J=7.5 \mathrm{~Hz}, 2 \mathrm{H}), 1.17(\mathrm{t}, J=7.5 \mathrm{~Hz}, 3 \mathrm{H}), 0.35(\mathrm{~s}, 9 \mathrm{H}) .{ }^{13} \mathrm{C}$ NMR $(125$ $\left.\mathrm{Hz}, \mathrm{CDCl}_{3}\right): \delta=161.8,149.4,142.8,134.5,125.4,122.7,121.0,42.5,24.2,14.9,0.2$. HRMS (ESITOF) $\mathrm{m} / \mathrm{z}:[\mathrm{M}+\mathrm{H}]^{+}$Calcd for $\mathrm{C}_{14} \mathrm{H}_{21} \mathrm{OSi}$ 233.1356; Found 233.1359. FTIR $\left(\mathrm{KBr}, \mathrm{cm}^{-1}\right)$ : 3453.77 , $3417.16,1651.55,1634.10,1402.44,1384.95,471.39,436.71$.

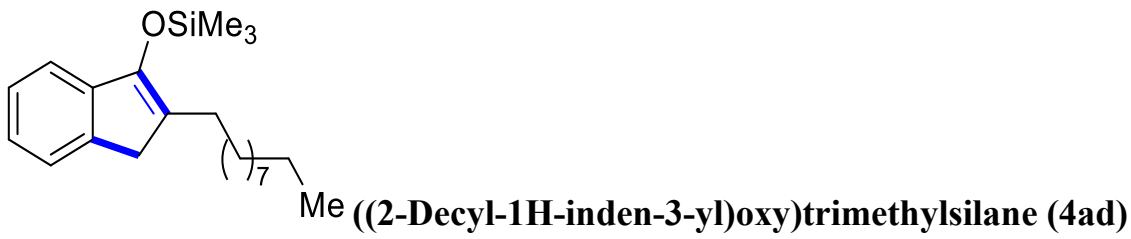

Following the general procedure, $\mathbf{4 a d}$ was obtained as a yellow oil by the purification with flash column chromatography on silica gel using PE as the eluent, $35.1 \mathrm{mg}$, yield $=51 \%$. ${ }^{1} \mathrm{H}$ NMR $(500$ $\left.\mathrm{MHz}, \mathrm{CDCl}_{3}\right): \delta=7.44-7.41(\mathrm{~m}, 1 \mathrm{H}), 7.39(\mathrm{~d}, \mathrm{~J}=7.5 \mathrm{~Hz}, 1 \mathrm{H}), 7.22-7.19(\mathrm{~m}, 1 \mathrm{H}), 7.10-7.06(\mathrm{~m}$, $1 \mathrm{H}), 3.39(\mathrm{~s}, 2 \mathrm{H}), 2.58-2.54(\mathrm{~m}, 2 \mathrm{H}), 1.53(\mathrm{~s}, 2 \mathrm{H}), 1.31-1.26(\mathrm{~m}, 14 \mathrm{H}), 0.89-0.86(\mathrm{~m}, 3 \mathrm{H}), 0.35(\mathrm{~d}$, $J=4.5 \mathrm{~Hz}, 9 \mathrm{H}) .{ }^{13} \mathrm{C} \mathrm{NMR}\left(125 \mathrm{~Hz}, \mathrm{CDCl}_{3}\right): \delta=160.6,149.3,142.8,134.8,125.3,122.6,122.6$, 120.9, 31.3, 31.2, 30.5, 29.3, 29.0, 28.8, 22.1, 13.5, 0.2. HRMS (ESI-TOF) m/z: $[\mathrm{M}+\mathrm{H}]^{+}$Calcd for $\mathrm{C}_{22} \mathrm{H}_{37} \mathrm{OSi}$ 345.2608; Found 345.2609. FTIR (KBr, $\left.\mathrm{cm}^{-1}\right)$ : 3444.37, 3417.69, 3224.58, 1651.39, $1644.67,1633.68,1402.64,1384.98$.

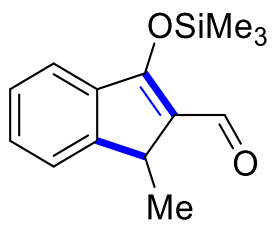

(S)-1-methyl-3-((trimethylsilyl)oxy)-1H-indene-2-carbaldehyde (3ae)

Following the general procedure, 3ae was obtained as a brown oil by the purification with flash 
column chromatography on silica gel (PE:EA $=200: 1), 33.5 \mathrm{mg}$, yield $=68 \% .{ }^{1} \mathrm{H}$ NMR $(500 \mathrm{MHz}$, $\left.\mathrm{CDCl}_{3}\right): \delta=10.25(\mathrm{~s}, 1 \mathrm{H}), 7.73(\mathrm{~d}, J=7.5 \mathrm{~Hz}, 1 \mathrm{H}), 7.50(\mathrm{~d}, J=32.0 \mathrm{~Hz}, 1 \mathrm{H}), 7.40-7.32(\mathrm{~m}, 2 \mathrm{H})$, $3.82(\mathrm{q}, J=7.5 \mathrm{~Hz}, 1 \mathrm{H}), 1.42(\mathrm{~d}, J=7.5 \mathrm{~Hz}, 3 \mathrm{H}), 0.50(\mathrm{~s}, 9 \mathrm{H}) .{ }^{13} \mathrm{C}$ NMR $\left(125 \mathrm{~Hz}, \mathrm{CDCl}_{3}\right): \delta=$ 188.6, 159.9, 158.1, 150.2, 144.5, 127.1, 125.6, 124.2, 122.3, 43.6, 15.6, 0.2. HRMS (ESI-TOF) $\mathrm{m} / \mathrm{z}:[\mathrm{M}+\mathrm{H}]^{+}$Calcd for $\mathrm{C}_{14} \mathrm{H}_{19} \mathrm{O}_{2} \mathrm{Si}$ 247.1149; Found 247.1151. FTIR $\left(\mathrm{KBr}, \mathrm{cm}^{-1}\right)$ : 3444.57, $3417.74,3159.92,2358.08,1651.59,1402.60,1251.77,841.25$.

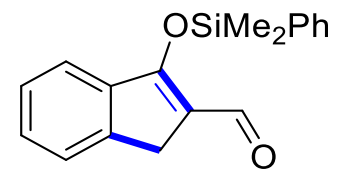

\section{3-((Dimethyl(phenyl)silyl)oxy)-1H-indene-2-carbaldehyde (3ua)}

Following the general procedure, 3ua was obtained as a yellow oil by the purification with flash column chromatography on silica gel (PE:EA $=100: 1), 32.4 \mathrm{mg}$, yield $=55 \% .{ }^{1} \mathrm{H}$ NMR $(500 \mathrm{MHz}$, $\left.\mathrm{CDCl}_{3}\right): \delta=9.96(\mathrm{~s}, 1 \mathrm{H}), 7.58-7.57(\mathrm{~m}, 1 \mathrm{H}), 7.55(\mathrm{~d}, J=7.5 \mathrm{~Hz}, 1 \mathrm{H}), 7.50(\mathrm{~d}, J=8.0 \mathrm{~Hz}, 1 \mathrm{H}), 7.41-$ $7.37(\mathrm{~m}, 2 \mathrm{H}), 7.33(\mathrm{t}, J=7.0 \mathrm{~Hz}, 1 \mathrm{H}), 7.25-7.22(\mathrm{~m}, 1 \mathrm{H}), 3.75(\mathrm{~s}, 2 \mathrm{H}), 0.75(\mathrm{~s}, 6 \mathrm{H}) .{ }^{13} \mathrm{C}$ NMR $(125$ $\left.\mathrm{Hz}, \mathrm{CDCl}_{3}\right): \delta=189.9,159.7,155.8,147.4,144.6,137.3,133.9,129.9,128.4,128.2,126.7,125.7$, 124.5, 38.6, 0.2. HRMS (ESI-TOF) m/z: $[\mathrm{M}+\mathrm{H}]^{+} \mathrm{Calcd}$ for $\mathrm{C}_{18} \mathrm{H}_{19} \mathrm{O}_{2} \mathrm{Si} 295.1149$; Found 295.1144. FTIR (KBr, $\left.\mathrm{cm}^{-1}\right): 3456.29,3417.61,2935.88,2851.09,2361.94,2339.90,1658.84,1402.23$.

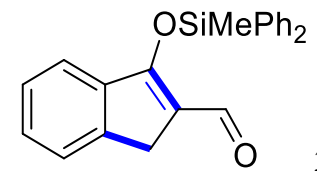

\section{3-((Methyldiphenylsilyl)oxy)-1H-indene-2-carbaldehyde (3va)}

Following the general procedure, 3va was obtained as a yellow oil by the purification with flash column chromatography on silica gel (PE:EA $=160: 1), 48.4 \mathrm{mg}$, yield $=68 \% .{ }^{1} \mathrm{H}$ NMR $(500 \mathrm{MHz}$, $\left.\mathrm{CDCl}_{3}\right): \delta=9.54(\mathrm{~s}, 1 \mathrm{H}), 7.58-7.54(\mathrm{~m}, 5 \mathrm{H}), 7.45-7.42(\mathrm{~m}, 2 \mathrm{H}), 7.39-7.36(\mathrm{~m}, 4 \mathrm{H}), 7.32-7.29(\mathrm{~m}$, 1H), $7.22(\mathrm{~d}, J=8.0 \mathrm{~Hz}, 1 \mathrm{H}), 7.13(\mathrm{t}, J=7.5 \mathrm{~Hz}, 1 \mathrm{H}), 3.79(\mathrm{~s}, 2 \mathrm{H}), 1.02(\mathrm{~s}, 3 \mathrm{H}) .{ }^{13} \mathrm{C}$ NMR $(125$ $\left.\mathrm{Hz}, \mathrm{CDCl}_{3}\right): \delta=191.8,159.2,158.2,149.0,146.0,136.8,136.4,131.7,130.0,129.7,128.2,127.7$, 125.9, 40.3, 0.2. HRMS (ESI-TOF) $\mathrm{m} / \mathrm{z}$ : $[\mathrm{M}+\mathrm{H}]^{+} \mathrm{Calcd}$ for $\mathrm{C}_{23} \mathrm{H}_{21} \mathrm{O}_{2} \mathrm{Si} 357.1305$; Found 357.1307. FTIR (KBr, $\left.\mathrm{cm}^{-1}\right): 3443.58,3416.79,1682.25,1659.24,1651.52,1634.20,1634.20,1402.54$. 


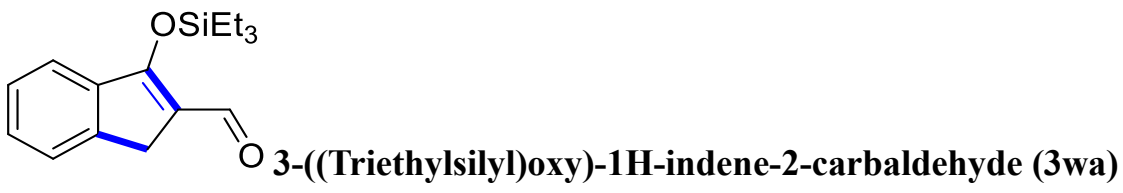

Following the general procedure, 3wa was obtained as a pale yellow oil by the purification with flash column chromatography on silica gel $(\mathrm{PE}: \mathrm{EA}=160: 1), 36.2 \mathrm{mg}$, yield $=66 \% .{ }^{1} \mathrm{H} \mathrm{NMR}(500$ $\left.\mathrm{MHz}, \mathrm{CDCl}_{3}\right): \delta=10.18(\mathrm{~d}, J=2.0 \mathrm{~Hz}, 1 \mathrm{H}), 7.77(\mathrm{~d}, J=7.5 \mathrm{~Hz}, 1 \mathrm{H}), 7.56(\mathrm{~d}, J=6.5 \mathrm{~Hz}, 1 \mathrm{H})$, 7.38-7.33 (m, 2H), $3.75(\mathrm{~s}, 2 \mathrm{H}), 1.02(\mathrm{~d}, J=2.0 \mathrm{~Hz}, 15 \mathrm{H}) .{ }^{13} \mathrm{C} \mathrm{NMR}\left(125 \mathrm{~Hz}, \mathrm{CDCl}_{3}\right): \delta=185.1$, 154.8, 151.4, 142.8, 139.7, 123.2, 121.7, 120.3, 119.5, 33.6, 2.5, 0.2. HRMS (ESI-TOF) m/z: [M + $\mathrm{H}]^{+}$Calcd for $\mathrm{C}_{16} \mathrm{H}_{23} \mathrm{O}_{2} \mathrm{Si} 275.1462$; Found 275.1462. FTIR (KBr, cm $\left.{ }^{-1}\right)$ : 3455.33, 3433.69, 3206.49, 1679.61, $1649.52,1642.94,1537.47$.<smiles>CCOC(=O)C=Cc1ccccc1C(C)=O</smiles>

\section{Ehyl (E)-3-(2-((trimethylsilyl)carbonyl)phenyl)acrylate (5af)}

Following the general procedure, 5af was obtained as a yellow oil by the purification with flash column chromatography on silica gel $(\mathrm{PE}: \mathrm{EA}=50: 1), 9.9 \mathrm{mg}$, yield $=19 \% .{ }^{1} \mathrm{H} \mathrm{NMR}(500 \mathrm{MHz}$, $\left.\mathrm{CDCl}_{3}\right): \delta=7.68(\mathrm{~d}, J=16.5 \mathrm{~Hz}, 1 \mathrm{H}), 7.65-7.61(\mathrm{~m}, 2 \mathrm{H}), 7.51-7.47(\mathrm{~m}, 2 \mathrm{H}), 6.53(\mathrm{~d}, J=16.0 \mathrm{~Hz}$, 1H), $2.73(\mathrm{q}, J=7.5 \mathrm{~Hz}, 2 \mathrm{H}), 1.17(\mathrm{t}, J=7.5 \mathrm{~Hz}, 3 \mathrm{H}), 0.33(\mathrm{~s}, 9 \mathrm{H}) .{ }^{13} \mathrm{C} \mathrm{NMR}\left(125 \mathrm{~Hz}, \mathrm{CDCl}_{3}\right): \delta$ $=243.7,203.1,144.5,143.5,134.7,133.2,131.3,131.3,131.0,130.1,35.2,10.1,0.2$.

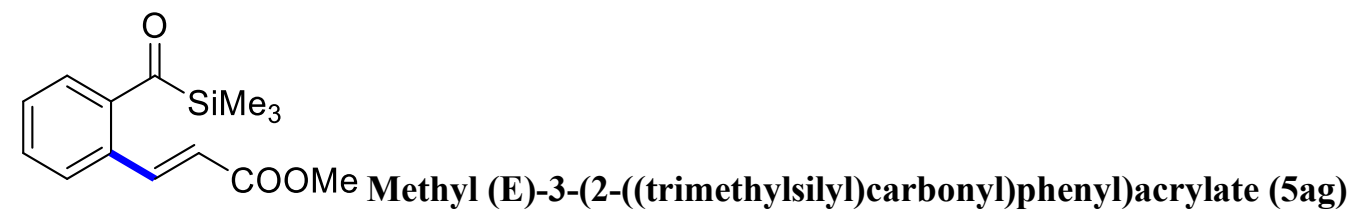

Following the general procedure, 5ag was obtained as a yellow oil by the purification with flash column chromatography on silica gel $(\mathrm{PE}: \mathrm{EA}=40: 1), 16.3 \mathrm{mg}$, yield $=31 \% .{ }^{1} \mathrm{H} \mathrm{NMR}(500 \mathrm{MHz}$, $\left.\mathrm{CDCl}_{3}\right): \delta=7.95(\mathrm{~d}, J=16.0 \mathrm{~Hz}, 1 \mathrm{H}), 7.60-7.57(\mathrm{~m}, 2 \mathrm{H}), 7.50-7.46(\mathrm{~m}, 2 \mathrm{H}), 6.28(\mathrm{~d}, J=16.0 \mathrm{~Hz}$, $1 \mathrm{H}), 3.81(\mathrm{~d}, J=1.5 \mathrm{~Hz}, 3 \mathrm{H}), 0.32(\mathrm{~d}, J=1.0 \mathrm{~Hz}, 9 \mathrm{H}) .{ }^{13} \mathrm{C} \mathrm{NMR}\left(125 \mathrm{~Hz}, \mathrm{CDCl}_{3}\right): \delta=243.9,169.0$, $145.8,145.1,134.4,133.1,131.5,130.8,130.2,122.5,53.8,0.2$. 
<smiles>CCOC=Cc1ccccc1C(=O)[SiH2]CC</smiles>

2 Diethyl (E)-(2-((trimethylsilyl)carbonyl)styryl)phosphonate (5ah)

Following the general procedure, 5ai was obtained as a yellow oil by the purification with flash column chromatography on silica gel $(\mathrm{PE}: \mathrm{EA}=1: 1), 46.9 \mathrm{mg}$, yield = 69\%. ${ }^{1} \mathrm{H} \mathrm{NMR}(500 \mathrm{MHz}$, $\left.\mathrm{CDCl}_{3}\right): \delta=7.71-7.64(\mathrm{dd}, J=22.5 \mathrm{~Hz}, J=17.5 \mathrm{~Hz}, 1 \mathrm{H}), 7.59-7.54(\mathrm{~m}, 2 \mathrm{H}), 7.48-7.46(\mathrm{~m}, 2 \mathrm{H})$, 6.15-6.07 (m, 1H), 4.20-4.14 (m, 4H), 1.38-1.35 (td, $J=7.0 \mathrm{~Hz}, J=1.5 \mathrm{~Hz}, 6 \mathrm{H}), 0.32-0.31(\mathrm{~m}, 9 \mathrm{H})$. ${ }^{13} \mathrm{C} \mathrm{NMR}\left(125 \mathrm{~Hz}, \mathrm{CDCl}_{3}\right): \delta=243.9,149.3\left(\mathrm{~d}, J_{\mathrm{C}-\mathrm{P}}=7.1 \mathrm{~Hz}\right), 144.8,135.0\left(\mathrm{~d}, J_{\mathrm{C}-\mathrm{P}}=23.8 \mathrm{~Hz}\right)$, $133.1,131.3,130.7,130.0\left(\mathrm{~d}, J_{\mathrm{C}-\mathrm{P}}=1.3 \mathrm{~Hz}\right), 119.7,118.2,64.1\left(\mathrm{~d}, J_{\mathrm{C}-\mathrm{P}}=5.5 \mathrm{~Hz}\right), 18.4\left(\mathrm{~d}, J_{\mathrm{C}-\mathrm{P}}=\right.$ $6.5 \mathrm{~Hz}), 0.2$.<smiles>CSC(=O)c1ccccc1/C=C/S(=O)(=O)c1ccccc1</smiles>

(E)-(2-(2-(phenylsulfonyl)vinyl)phenyl)(trimethylsilyl)methanone (5ai)

Following the general procedure, 5aj was obtained as a yellow oil by the purification with flash column chromatography on silica gel $(\mathrm{PE}: \mathrm{EA}=2: 1), 54.4 \mathrm{mg}$, yield $=79 \% .{ }^{1} \mathrm{H} \mathrm{NMR}(500 \mathrm{MHz}$, $\left.\mathrm{CDCl}_{3}\right): \delta=8.05-8.03(\mathrm{~m}, 2 \mathrm{H}), 7.97(\mathrm{~d}, J=15 \mathrm{~Hz}, 1 \mathrm{H}), 7.68(\mathrm{~d}, J=7.5 \mathrm{~Hz}, 1 \mathrm{H}), 7.62-7.48(\mathrm{~m}, 6 \mathrm{H})$, $6.69(\mathrm{~d}, J=15.0 \mathrm{~Hz}, 1 \mathrm{H}), 0.34(\mathrm{~s}, 9 \mathrm{H}) .{ }^{13} \mathrm{C} \mathrm{NMR}\left(125 \mathrm{~Hz}, \mathrm{CDCl}_{3}\right): \delta=242.6,144.7,144.7,142.6$, $135.3,133.3,132.4,132.0,131.5,131.2,131.1,130.6,129.7,0.2$.

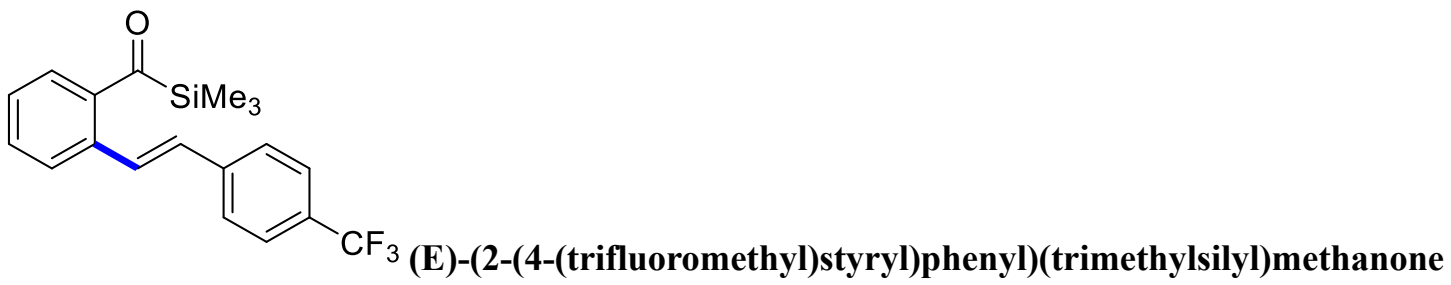
(5aj)

Following the general procedure, 5ak was obtained as a yellow solid by the purification with flash column chromatography on silica gel $(\mathrm{PE}: \mathrm{EA}=160: 1)$, m.p.: $70.3-71.7^{\circ} \mathrm{C}, 36.9 \mathrm{mg}$, yield $=53 \%$. ${ }^{1} \mathrm{H}$ NMR $\left(500 \mathrm{MHz}, \mathrm{CDCl}_{3}\right): \delta=7.67(\mathrm{~d}, J=8.0 \mathrm{~Hz}, 1 \mathrm{H}), 7.61-7.58(\mathrm{~m}, 5 \mathrm{H}), 7.56-7.54(\mathrm{dd}, J=8.0$ $\mathrm{Hz}, J=1.5 \mathrm{~Hz}, 1 \mathrm{H}), 7.45(\mathrm{t}, J=7.5 \mathrm{~Hz}, 1 \mathrm{H}), 7.40(\mathrm{t}, J=7.0 \mathrm{~Hz}, 1 \mathrm{H}), 6.91(\mathrm{~d}, J=16.0 \mathrm{~Hz}, 1 \mathrm{H})$, $0.30(\mathrm{~s}, 9 \mathrm{H}) .{ }^{13} \mathrm{C} \mathrm{NMR}\left(125 \mathrm{~Hz}, \mathrm{CDCl}_{3}\right): \delta=244.8,144.0,142.7,136.5,132.9,132.3,131.8,131.4$ 
$\left(\mathrm{q}, J_{\mathrm{C}-\mathrm{F}}=32.3 \mathrm{~Hz}\right), 130.9,129.7,129.4,128.8,127.6\left(\mathrm{q}, J_{\mathrm{C}-\mathrm{F}}=3.8 \mathrm{~Hz}\right), 126.2\left(\mathrm{q}, J_{\mathrm{C}-\mathrm{F}}=270.1 \mathrm{~Hz}\right)$.<smiles>COC1=CCc2ccccc21</smiles>

(1H-Inden-3-yl)oxy)trimethylsilane (4aa)

Following the general procedure, 4aa was obtained as a pale yellow oil by the purification with flash column chromatography on silica gel using PE as the eluent, $1.2 \mathrm{mg}$, yield $=3 \%$. ${ }^{1} \mathrm{H}$ NMR $\left(500 \mathrm{MHz}, \mathrm{CDCl}_{3}\right): \delta=7.39-7.36(\mathrm{~m}, 2 \mathrm{H}), 7.18-7.14(\mathrm{~m}, 1 \mathrm{H}), 7.08-7.04(\mathrm{~m}, 1 \mathrm{H}), 6.65-6.63(\mathrm{~m}$, $1 \mathrm{H}), 3.29(\mathrm{~d}, \mathrm{~J}=2.5 \mathrm{~Hz}, 2 \mathrm{H}), 0.22-0.19(\mathrm{~m}, 9 \mathrm{H}) .{ }^{13} \mathrm{C} \mathrm{NMR}\left(125 \mathrm{~Hz}, \mathrm{CDCl}_{3}\right): \delta=149.4,146.5$, 146.0, 145.4, 127.4, 125.6, 125.1, 123.3, 42.0, $0.2(\mathrm{~d}, J=3.0 \mathrm{~Hz})$. HRMS (ESI-TOF) m/z: $[\mathrm{M}+\mathrm{H}]^{+}$ Calcd for $\mathrm{C}_{12} \mathrm{H}_{17} \mathrm{OSi}$ 205.1043; Found 205.1042. FTIR (KBr, $\left.\mathrm{cm}^{-1}\right)$ : 3493.29, 3416.36, 3300.24, $2367.89,2289.63,1658.81,1643.78,1632.84$.<smiles>COC(=O)c1ccccc1/C=C/C=O</smiles>

\section{(E)-3-(2-((trimethylsilyl)carbonyl)phenyl)acrylaldehyde (5aa)}

Following the general procedure, 5aa was obtained as a deep yellow solid by the purification with flash column chromatography on silica gel $(\mathrm{PE}: \mathrm{EA}=60: 1)$, m.p.: $53.6-54.4{ }^{\circ} \mathrm{C}, 1.0 \mathrm{mg}$, yield $=2 \%$. ${ }^{1} \mathrm{H}$ NMR (500 MHz, $\left.\mathrm{CDCl}_{3}\right): \delta=9.73(\mathrm{~d}, J=8.0 \mathrm{~Hz}, 1 \mathrm{H}), 7.93(\mathrm{~d}, J=16.0 \mathrm{~Hz}, 1 \mathrm{H}), 7.76-7.74(\mathrm{~m}$, 1H), 7.66-7.65 (m, 1H), 7.58-7.52 (m, 2H), $6.58(\mathrm{q}, J=8.0 \mathrm{~Hz}, 1 \mathrm{H}), 0.36(\mathrm{~s}, 9 \mathrm{H}) .{ }^{13} \mathrm{C}$ NMR $(125$ $\left.\mathrm{Hz}, \mathrm{CDCl}_{3}\right): \delta=243.2,196.0,153.6,143.9,133.9,133.4,132.7,132.0,132.0,130.3,0.2 . \mathrm{HRMS}$ (ESI-TOF) m/z: $[\mathrm{M}+\mathrm{H}]^{+}$Calcd for $\mathrm{C}_{13} \mathrm{H}_{17} \mathrm{O}_{2} \mathrm{Si}$ 233.0992; Found 233.0997. FTIR $\left(\mathrm{KBr}, \mathrm{cm}^{-1}\right)$ : 3443.12, 3422.59, 2923.97, 2850.63, 2354.42, 1643.25, 1632.39, 1402.04 . 


\section{Controlled Experiments}

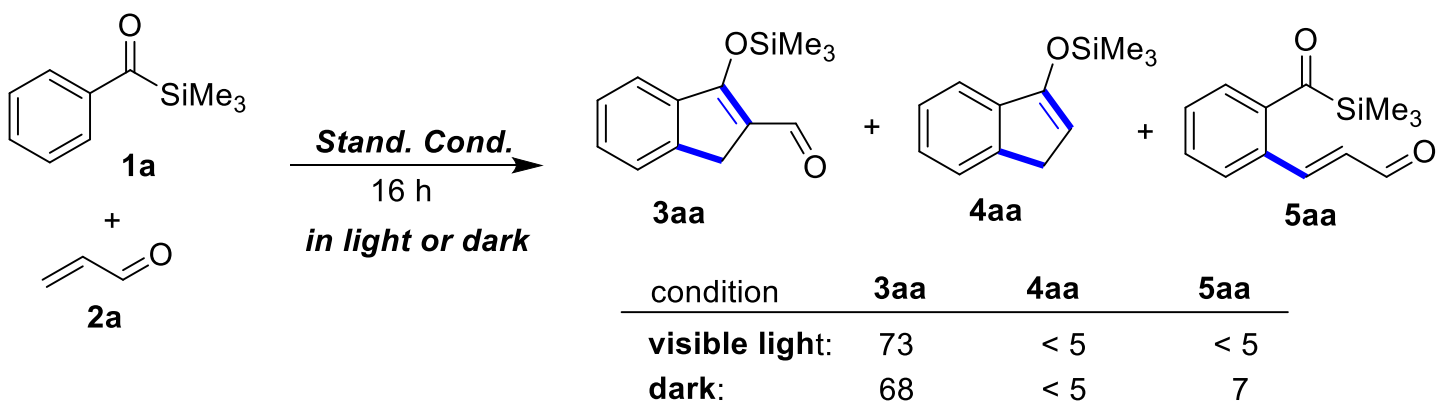

A screw-cap vial was charged with $\left[\mathrm{Ru}(p \text {-cymene }) \mathrm{Cl}_{2}\right]_{2}(5 \mathrm{~mol} \%, 0.01 \mathrm{mmol}), \mathrm{AgSbF}_{6}(20$ mol\%, $0.04 \mathrm{mmol}), \mathrm{Cu}(\mathrm{OAc})_{2}(1.3$ equiv, $0.26 \mathrm{mmol})$ and $\mathrm{DCM}(1.0 \mathrm{~mL})$. Then, aroylsilane $1 \mathrm{a}$ (1.0 equiv, $0.2 \mathrm{mmol}$ ) and $2 \mathrm{a}(3.0$ equiv, $0.6 \mathrm{mmol})$ were added into the solution in sequence. The vial was sealed under argon and heated to $60{ }^{\circ} \mathrm{C}$ in an oil bath under visible light or in dark. After stirring for $16 \mathrm{~h}$, the mixture was cooling down and directly applied to a flash column chromatography (3aa, PE:EA = 160:1; 4aa, PE; 5aa, PE:EA = 60:1).<smiles>CS(=O)(=O)C(=O)c1ccccc1/C=C/C=O</smiles>

5 aa

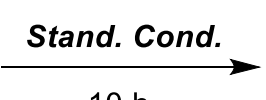

$10 \mathrm{~h}$

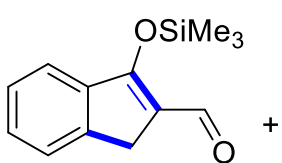

3aa, $0 \%$<smiles>COC1=CCc2ccccc21</smiles>

4aa, $0 \%$<smiles>CC(=O)c1ccccc1/C=C/C=O</smiles>

5aa, $43 \%$ recovered

A screw-cap vial was charged with $\left[\mathrm{Ru}(p \text {-cymene }) \mathrm{Cl}_{2}\right]_{2}(5 \mathrm{~mol} \%, 0.005 \mathrm{mmol}), \mathrm{AgSbF}_{6}(20$ mol\%, $0.02 \mathrm{mmol}), \mathrm{Cu}(\mathrm{OAc})_{2}(1.3$ equiv , $0.13 \mathrm{mmol})$ and $\mathrm{DCM}(0.5 \mathrm{~mL})$. Then, $5 \mathbf{a a}$ (1.0 equiv, $0.1 \mathrm{mmol}$ ) was added into the solution in sequence. The vial was sealed under argon and heated to $60{ }^{\circ} \mathrm{C}$ in an oil bath with stirring for $10 \mathrm{~h}$. After cooling down, the mixture was directly applied to a flash column chromatography (PE:EA $=60: 1)$, leading to no cyclization products 3aa or 4aa, only with the $43 \%$ recovery of $\mathbf{5 a a}, 10.0 \mathrm{mg}$.<smiles>COC1c2ccccc2CC1C</smiles>
6<smiles>COC1c2ccccc2CC1(C)C=O</smiles>

7

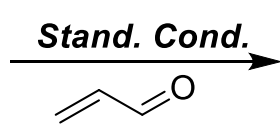

2a<smiles>COC1=C(C)Cc2ccccc21</smiles>

$4 a b, 0 \%$

A screw-cap vial was charged with $\left[\mathrm{Ru}(p \text {-cymene }) \mathrm{Cl}_{2}\right]_{2}(5 \mathrm{~mol} \%, 0.005 \mathrm{mmol}), \mathrm{AgSbF}_{6}(20 \mathrm{~mol} \%$, 
$0.02 \mathrm{mmol}), \mathrm{Cu}(\mathrm{OAc})_{2}(1.3$ equiv , $0.13 \mathrm{mmol})$ and $\mathrm{DCM}(0.5 \mathrm{~mL})$. Then, 6 or 7 (1.0 equiv, 0.1 mmol) with or without $\mathbf{2 b}$ (3.0 equiv, $0.3 \mathrm{mmol}, 16.8 \mathrm{mg}$ ) were added into the solution in sequence. The vial was sealed under argon and heated to $60^{\circ} \mathrm{C}$ in an oil bath with stirring for $10 \mathrm{~h}$. After cooling down, no product $\mathbf{4 a b}$ was obtained.

\section{Synthesis of Compound 6}<smiles>O=C1CCc2ccccc21</smiles>

A

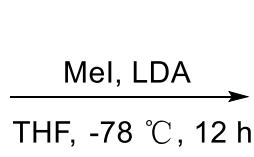

THF, $-78{ }^{\circ} \mathrm{C}, 12 \mathrm{~h}$<smiles>CC1Cc2ccccc2C1=O</smiles>

B

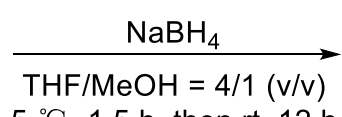

$5{ }^{\circ} \mathrm{C}, 1.5 \mathrm{~h}$, then rt. $12 \mathrm{~h}$<smiles>CC1Cc2ccccc2C1O</smiles>

C

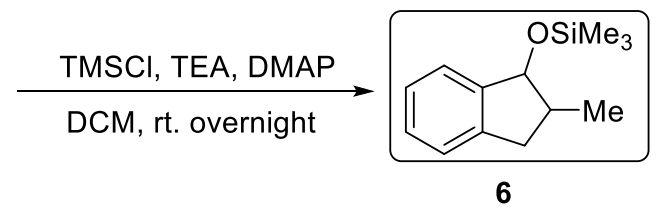

A $(1.0 \mathrm{~g}, 7.57 \mathrm{mmol})$ was added dropwisely to a stirred solution of LDA $(3.8 \mathrm{~mL}, 2.0 \mathrm{M}$ in THF, $7.57 \mathrm{mmol})$ in THF $(25 \mathrm{~mL})$ at $-78{ }^{\circ} \mathrm{C}$ and stirred for 20 minutes. MeI $(1.074 \mathrm{~g}, 7.57 \mathrm{mmol})$ was then added this solution was stirred at room temperature for $12 \mathrm{~h}$. A solution of $\mathrm{NH}_{4} \mathrm{Cl}$ was added and the mixture was extracted with EtOAc. The combined organic layers were dried over $\mathrm{Na}_{2} \mathrm{SO}_{4}$, and then concentrated in vacuo. The residue was applied to flash chromatography (PE:EA = 80:1) to afford product $\mathbf{B}(332.0 \mathrm{mg}, 30 \%)$ as a pale yellow oil. ${ }^{[12]}{ }^{1} \mathrm{H} \mathrm{NMR}\left(500 \mathrm{MHz}, \mathrm{CDCl}_{3}\right): \delta=7.74$ $(\mathrm{d}, J=7.5 \mathrm{~Hz}, 1 \mathrm{H}), 7.57(\mathrm{t}, J=7.5 \mathrm{~Hz}, 1 \mathrm{H}), 7.44(\mathrm{~d}, J=7.5 \mathrm{~Hz}, 1 \mathrm{H}), 7.35(\mathrm{t}, J=7.5 \mathrm{~Hz}, 1 \mathrm{H}), 3.38$ $(\mathrm{q}, J=8.5 \mathrm{~Hz}, 1 \mathrm{H}), 2.73-2.66(\mathrm{~m}, 2 \mathrm{H}), 1.31-1.29(\mathrm{~m}, 3 \mathrm{H}) .{ }^{13} \mathrm{C} \mathrm{NMR}\left(125 \mathrm{~Hz}, \mathrm{CDCl}_{3}\right): \delta=209.3$, $153.4,136.3,134.6,127.3,126.5,123.8,41.9,34.9,16.2$.

$\mathrm{NaBH}_{4}$ (128.6 mg, $3.4 \mathrm{mmol}$ ) was added dropwise to a stirred solution of $\mathbf{B}$ (332.0 mg, 2.27 mmol) in $2.1 \mathrm{~mL}$ of THF/Methanol (in a ratio of $4: 1$ by volume) at $0{ }^{\circ} \mathrm{C}$ and stirred for 5 minutes. This mixture was stirred at room temperature for $12 \mathrm{~h}$. water was added at $0{ }^{\circ} \mathrm{C}$ and the mixture was extracted with $\mathrm{CH}_{2} \mathrm{Cl}_{2}$. The combined organic layers were dried over $\mathrm{Na}_{2} \mathrm{SO}_{4}$, and then concentrated in vacuo. The residue was applied to flash chromatography (PE:EA=3:1) to afford product $\mathbf{C}(269.0$ $\mathrm{mg}, 80 \%, 1: 0.54$ d.r.) as a white solid, m.p.: $61.4-63.8^{\circ} \mathrm{C} .{ }^{[13]}{ }^{1} \mathrm{H} \mathrm{NMR}\left(500 \mathrm{MHz}, \mathrm{CDCl}_{3}\right): \delta=7.39$ $(\mathrm{d}, J=7.0 \mathrm{~Hz}, 0.54 \mathrm{H}), 7.36-7.33(\mathrm{~m}, 0.98 \mathrm{H}), 7.25-7.17(\mathrm{~m}, 4.66 \mathrm{H}), 4.98(\mathrm{~m}, J=5.5 \mathrm{~Hz}, 0.54 \mathrm{H})$, $4.69(\mathrm{~m}, J=6.5 \mathrm{~Hz}, 1 \mathrm{H}), 3.09(\mathrm{dd}, J=15.5 \mathrm{~Hz}, J=7.5 \mathrm{~Hz}, 1 \mathrm{H}), 2.94(\mathrm{dd}, J=15.5 \mathrm{~Hz}, J=7.0 \mathrm{~Hz}$, 
$0.54 \mathrm{H}), 2.67(\mathrm{dd}, J=15.5 \mathrm{~Hz}, J=7.0 \mathrm{~Hz}, 0.54 \mathrm{H}), 2.57-2.50(\mathrm{~m}, 0.54 \mathrm{H}), 2.45(\mathrm{dd}, J=15.5 \mathrm{~Hz}, J=$ $8.0 \mathrm{~Hz}, 1 \mathrm{H}), 2.27-2.18(\mathrm{~m}, 1 \mathrm{H}), 1.24(\mathrm{~d}, J=6.5 \mathrm{~Hz}, 3 \mathrm{H}), 1.12(\mathrm{~d}, J=7.0 \mathrm{~Hz}, 1.62 \mathrm{H}) .{ }^{13} \mathrm{C}$ NMR $\left(125 \mathrm{~Hz}, \mathrm{CDCl}_{3}\right): \delta=144.0,143.7,142.3,141.0,127.4,127.0,125.6,123.9,123.7,123.7,122.9$, $81.8,76.6,44.4,38.2,36.9,36.8,16.7,12.6$.

TMSCl (276.8 mg, $2.55 \mathrm{mmol}$ ) was added dropwise to a stirred solution of $\mathbf{C}$ (269.0 mg, 1.82 mmol), TEA (184.2 mg, $1.82 \mathrm{mmol})$ and DMAP (133.4 $\mathrm{mg}, 1.09 \mathrm{mmol})$ in DCM (4 mL) at $0{ }^{\circ} \mathrm{C}$ and stirred for 5 minutes. This mixture was stirred at room temperature for $12 \mathrm{~h}$. water was added at $0{ }^{\circ} \mathrm{C}$ and the mixture was extracted with $\mathrm{CH}_{2} \mathrm{Cl}_{2}$. The combined organic layers were dried over $\mathrm{Na}_{2} \mathrm{SO}_{4}$, and then concentrated in vacuo. The residue was applied to flash chromatography (PE:EA $=600: 1)$ to provide compound $6\left(297.0 \mathrm{mg}, 80 \%, 1: 0.56\right.$ d.r.) as a colorless oil. ${ }^{1} \mathrm{H}$ NMR $(500 \mathrm{MHz}$, $\left.\mathrm{CDCl}_{3}\right): \delta=7.27-7.22(\mathrm{~m}, 1.53 \mathrm{H}), 7.19-7.12(\mathrm{~m}, 4.67 \mathrm{H}), 5.05(\mathrm{~d}, J=5.5 \mathrm{~Hz}, 0.55 \mathrm{H}), 4.73(\mathrm{~d}, J=$ $7.0 \mathrm{~Hz}, 0.97 \mathrm{H}), 3.04$ (dd, $J=15.0 \mathrm{~Hz}, J=8.0 \mathrm{~Hz}, 1 \mathrm{H}), 2.89$ (dd, $J=15.5 \mathrm{~Hz}, J=7.0 \mathrm{~Hz}, 0.56 \mathrm{H}$ ), $2.64(\mathrm{dd}, J=15.5 \mathrm{~Hz}, J=5.5 \mathrm{~Hz}, 0.56 \mathrm{H}), 2.52-2.45(\mathrm{~m}, 0.56 \mathrm{H}), 2.42(\mathrm{dd}, J=15.5 \mathrm{~Hz}, J=9.0 \mathrm{~Hz}$, 1H), 2.33-2.24 (m, 1H), 1.20 (d, $J=7.0 \mathrm{~Hz}, 3 \mathrm{H}), 1.01$ (d, $J=7.0 \mathrm{~Hz}, 3 \mathrm{H}), 0.23$ (s, 9H), 0.19 (s, 5.10H). ${ }^{13} \mathrm{C} \mathrm{NMR}\left(125 \mathrm{~Hz}, \mathrm{CDCl}_{3}\right): \delta=145.3,144.8,142.4,141.5,127.6,127.5,126.4,126.3$, 124.8, 124.4, 124.4, 123.7, 82.8, 77.5, 45.0, 39.7, 37.9, 37.6, 17.6, 14.0, 0.5, 0.2. HRMS (ESI-TOF) $\mathrm{m} / \mathrm{z}:[\mathrm{M}+\mathrm{H}]^{+}$Calcd for $\mathrm{C}_{13} \mathrm{H}_{21} \mathrm{OSi} 221.1356$; Found 221.1352. FTIR $\left(\mathrm{KBr}, \mathrm{cm}^{-1}\right): 3444.81,3418.09$, 3132.65, 1651.69, 1621.75, 1615.04, 1505.39, 1455.52, 1398.46.

\section{Synthesis of Compound 7}<smiles>O=C1c2ccccc2CC1[N+](=O)[O-]</smiles>

B

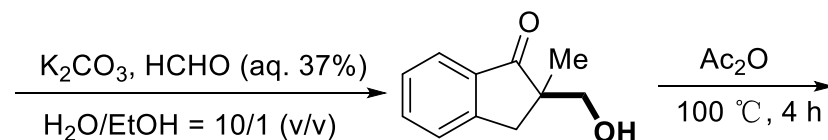

$\mathbf{C}^{\prime}$<smiles>CC(=O)OCC1(C)Cc2ccccc2C1=O</smiles>

D<smiles>COC1c2ccccc2CC1(COC(C)=O)C(C)(C)C</smiles>

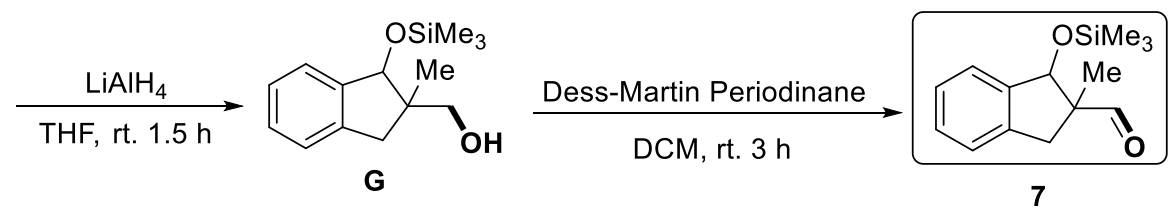


Compound $\mathbf{B}(1.77 \mathrm{~g}, 8.35 \mathrm{~mol}), \mathrm{K}_{2} \mathrm{CO}_{3}(836.2 \mathrm{mg}, 4.18 \mathrm{~mol})$ was added to a round-bottomed flask and dissolved in $\mathrm{H}_{2} \mathrm{O} / \mathrm{EtOH}$ (in a ratio of $10: 1$ by volume) to make a $0.04 \mathrm{M}$ solution. Formaldehyde ( $37 \%$ aqueous solution, $134.5 \mathrm{~mL}$ ) was added at $40{ }^{\circ} \mathrm{C}$ in an oil bath and the mixture was maintained for over $1 \mathrm{~h}$. Then $1.0 \mathrm{M}$ hydrochloric acid was added at $0{ }^{\circ} \mathrm{C}$ and the mixture was extracted with $\mathrm{CH}_{2} \mathrm{Cl}_{2}$. The combined organic layers were dried over $\mathrm{Na}_{2} \mathrm{SO}_{4}$, and then concentrated in vacuo. The residue was applied to flash chromatography on silica gel (PE:EA $=8: 1)$ to afford product $\mathbf{C}^{\prime}(1.37$ $\mathrm{g}, 93 \%)$ as a colorless oil. ${ }^{[14]}{ }^{1} \mathrm{H} \mathrm{NMR}\left(500 \mathrm{MHz}, \mathrm{CDCl}_{3}\right): \delta=7.68(\mathrm{~d}, J=8.0 \mathrm{~Hz}, 1 \mathrm{H}), 7.57(\mathrm{t}, J=$ $7.5 \mathrm{~Hz}, 1 \mathrm{H}), 7.44(\mathrm{~d}, J=8.0 \mathrm{~Hz}, 1 \mathrm{H}), 7.32(\mathrm{t}, J=7.5 \mathrm{~Hz}, 1 \mathrm{H}), 3.81(\mathrm{dd}, J=10.5 \mathrm{~Hz}, J=4.5 \mathrm{~Hz}$, 1H), 3.58 (d, $J=10.5 \mathrm{~Hz}, 1 \mathrm{H}), 3.30$ (d, $J=17.0 \mathrm{~Hz}, 1 \mathrm{H}), 3.11$ (s, 1H), 2.86 (d, $J=17.0 \mathrm{~Hz}, 1 \mathrm{H})$, 1.19 (s, 3H). ${ }^{13} \mathrm{C}$ NMR $\left(125 \mathrm{~Hz}, \mathrm{CDCl}_{3}\right): \delta=210.2,152.5,134.8,134.1,126.4,125.7,123.1,66.6$, $50.1,36.9,19.6$.

Acetic anhydride $(1.11 \mathrm{~g}, 7.77 \mathrm{mmol})$ was added in dropwise to a stirred solution of $\mathbf{C}^{\prime}(1.37 \mathrm{~g}, 7.77$ mmol) at $100{ }^{\circ} \mathrm{C}$ in an oil bath and stirred for $4 \mathrm{~h}$. water was added at $0{ }^{\circ} \mathrm{C}$. A solution of $\mathrm{NH}_{4} \mathrm{Cl}$ was added and the mixture was extracted with $\mathrm{CH}_{2} \mathrm{Cl}_{2}$. The combined organic layers were dried over $\mathrm{Na}_{2} \mathrm{SO}_{4}$, and then concentrated in vacuo. The residue was applied to flash chromatography on silica gel (PE:EA $=20: 1)$ to afford $\mathbf{D}(1.58 \mathrm{~g}, 93 \%)$ as a colorless oil. ${ }^{1} \mathrm{H}$ NMR $\left(500 \mathrm{MHz}, \mathrm{CDCl}_{3}\right)$ : $\delta=7.77(\mathrm{~d}, J=7.5 \mathrm{~Hz}, 1 \mathrm{H}), 7.62(\mathrm{td}, J=7.5 \mathrm{~Hz}, J=1.0 \mathrm{~Hz}, 1 \mathrm{H}), 7.47(\mathrm{~d}, J=7.5 \mathrm{~Hz}, 1 \mathrm{H}), 7.39(\mathrm{t}$, $J=7.5 \mathrm{~Hz}, 1 \mathrm{H}), 4.23(\mathrm{q}, J=10.5 \mathrm{~Hz}, J=5.5 \mathrm{~Hz}, 2 \mathrm{H}), 3.28(\mathrm{~d}, J=17.0 \mathrm{~Hz}, 1 \mathrm{H}), 2.93(\mathrm{~d}, J=17.0$ $\mathrm{Hz}, 1 \mathrm{H}), 1.91$ (s, 3H), 1.24 (s, 3H). ${ }^{13} \mathrm{C} \mathrm{NMR}\left(125 \mathrm{~Hz}, \mathrm{CDCl}_{3}\right): \delta=206.9,169.8,151.5,134.6$, 134.1, 126.6, 125.6, 123.4, 67.2, 47.8, 37.0, 20.1, 19.6. HRMS (ESI-TOF) m/z: $[\mathrm{M}+\mathrm{H}]^{+}$Calcd for $\mathrm{C}_{13} \mathrm{H}_{15} \mathrm{O}_{3}$ 219.1016; Found 219.1016. FTIR $\left(\mathrm{KBr}, \mathrm{cm}^{-1}\right): 3444.52,3160.55,3144.29,1644.71$, $1557.20,1434.69,1402.39,1384.96$.

Compound D (1.58 g, $7.23 \mathrm{~mol})$ was added to a round-bottomed flask and dissolved in THF/MeOH (in a ratio of 2:1 by volume) to make a $2.2 \mathrm{M}$ solution. $\mathrm{NaBH}_{4}(408.6 \mathrm{mg}, 10.8 \mathrm{~mol}$ ) was added at $0{ }^{\circ} \mathrm{C}$ and the mixture was stirred at room temperature overnight. Then water was added in dropwise at $0{ }^{\circ} \mathrm{C}$, and the mixture was extracted with $\mathrm{CH}_{2} \mathrm{Cl}_{2}$. The combined organic layers were dried over $\mathrm{Na}_{2} \mathrm{SO}_{4}$, and then concentrated in vacuo. The residue was applied to flash chromatography on silica gel (PE:EA = 15:1) to afford product $\mathbf{E}\left(1.06 \mathrm{~g}, 67 \%, 5: 1\right.$ d.r.) as a pale blue oil. ${ }^{1} \mathrm{H}$ NMR (500 MHz, $\left.\mathrm{CDCl}_{3}\right): \delta=7.36-7.34(\mathrm{~m}, 1.23 \mathrm{H}), 7.23-7.16(\mathrm{~m}, 3.57 \mathrm{H}), 4.97(\mathrm{~d}, J=5.5 \mathrm{~Hz}, 0.95 \mathrm{H}), 4.75(\mathrm{~d}, J=$ 
$6.0 \mathrm{~Hz}, 0.19 \mathrm{H}), 4.21(\mathrm{~s}, 0.4 \mathrm{H}), 4.13(\mathrm{~d}, J=11.0 \mathrm{~Hz}, 1 \mathrm{H}), 4.05(\mathrm{~d}, J=11.0 \mathrm{~Hz}, 1 \mathrm{H}), 3.01(\mathrm{~d}, J=16.0$ $\mathrm{Hz}, 0.2 \mathrm{H}), 2.86$ (d, $J=15.5 \mathrm{~Hz}, 1 \mathrm{H}), 2.67$ (d, $J=15.5 \mathrm{~Hz}, 1 \mathrm{H}), 2.60$ (d, $J=16.0 \mathrm{~Hz}, 0.2 \mathrm{H}), 2.04$ (s, $3 \mathrm{H}), 1.86(\mathrm{~s}, 0.6 \mathrm{H}), 1.13$ (s, 0.6H), 1.03 (s, 3H). ${ }^{13} \mathrm{C} \mathrm{NMR}\left(125 \mathrm{~Hz}, \mathrm{CDCl}_{3}\right): \delta=171.9,171.3,143.8$, $143.4,141.3,140.2,128.4,128.1,126.9,126.8,125.0,124.9,124.6,124.4,81.9,78.8,69.8,68.0$, 48.3, 47.6, 40.7, 40.4, 22.3, 20.9, 20.6, 17.2. HRMS (ESI-TOF) m/z: $[\mathrm{M}+\mathrm{H}]^{+}$Calcd for $\mathrm{C}_{13} \mathrm{H}_{17} \mathrm{O}_{3}$ 221.1172; Found 221.1170. FTIR (KBr, $\left.\mathrm{cm}^{-1}\right): 3444.77,3132.06,2357.44,1651.62,1557.42$, $1463.13,1398.54$.

Compound E (1.06 g, $4.84 \mathrm{~mol})$, TEA (489.8 mg, $4.84 \mathrm{~mol})$ and DMAP (354.8 mg, $2.9 \mathrm{~mol})$ was added to a round-bottomed flask and dissolved in dry DCM (17 mL). TMSCl (736.1 mg, $6.78 \mathrm{~mol})$ was added in dropwise at $0{ }^{\circ} \mathrm{C}$, and the mixture was stirred at room temperature overnight. Then water was added at $0{ }^{\circ} \mathrm{C}$ and the mixture was extracted with $\mathrm{CH}_{2} \mathrm{Cl}_{2}$. The combined organic layers were dried over $\mathrm{Na}_{2} \mathrm{SO}_{4}$ and then concentrated in vacuo. The residue was applied to flash chromatography on silica gel (PE:EA $=25: 1)$ to afford product $\mathbf{F}(1.17 \mathrm{~g}, 83 \%, 1: 0.42$ d.r.) as a colorless oil. ${ }^{1} \mathrm{H}$ NMR (500 MHz, $\left.\mathrm{CDCl}_{3}\right): \delta=7.24-7.14(\mathrm{~m}, 5.68 \mathrm{H}), 5.07(\mathrm{~s}, 0.99 \mathrm{H}), 4.82(\mathrm{~s}, 0.41 \mathrm{H})$, $4.18(\mathrm{~d}, J=11.0 \mathrm{~Hz}, 0.42 \mathrm{H}), 4.03$ (q, $J=11.5 \mathrm{~Hz}, 2.02 \mathrm{H}), 3.99(\mathrm{~d}, J=11.0 \mathrm{~Hz}, 0.42 \mathrm{H}), 3.01$ (d, $J$ $=15.5 \mathrm{~Hz}, 0.42 \mathrm{H}), 2.88(\mathrm{~d}, J=15.5 \mathrm{~Hz}, 1 \mathrm{H}), 2.66(\mathrm{~d}, J=15.5 \mathrm{~Hz}, 1 \mathrm{H}), 2.54(\mathrm{~d}, J=15.5 \mathrm{~Hz}, 0.42 \mathrm{H})$, $2.06(\mathrm{~s}, 3 \mathrm{H}), 2.03(\mathrm{~s}, 1.27 \mathrm{H}), 1.14(\mathrm{~s}, 1.25 \mathrm{H}), 0.98(\mathrm{~s}, 3 \mathrm{H}), 0.22(\mathrm{~s}, 9.03 \mathrm{H}), 0.19(\mathrm{~s}, 3.78 \mathrm{H}) .{ }^{13} \mathrm{C}$ NMR (125 Hz, $\left.\mathrm{CDCl}_{3}\right): \delta=171.8,171.6,144.9,144.5,141.4,140.6,128.5,128.3,127.3,127.1$, 125.7, 125.6, 125.0, 124.9, 83.1, 78.8, 69.5, 68.4, 49.0, 48.2, 40.9, 40.6, 22.6, 21.5, 21.4, 18.1, 1.0, 1.0. HRMS (ESI-TOF) $\mathrm{m} / \mathrm{z}$ : $[\mathrm{M}+\mathrm{H}]^{+}$Calcd for $\mathrm{C}_{16} \mathrm{H}_{25} \mathrm{O}_{3} \mathrm{Si}$ 293.1567; Found 293.1573. FTIR (KBr, $\left.\mathrm{cm}^{-1}\right): 3444.35,3161.84,3132.68,1651.47,1557.21,1538.23,1402.49,1385.08$.

A round-bottomed flask was charged with $\mathrm{LiAlH}_{4}(2.5 \mathrm{M}$ in THF, $1.9 \mathrm{~mL}, 4.82 \mathrm{~mol})$ and dry THF (35 mL). A solution of $\mathbf{F}(1.17 \mathrm{~g}, 4.02 \mathrm{mmol})$ in THF $(6 \mathrm{~mL})$ was added to it at $0{ }^{\circ} \mathrm{C}$. The mixture was stirred at room temperature over $1.5 \mathrm{~h}$. Then water was added at $0{ }^{\circ} \mathrm{C}$ and the mixture was extracted with $\mathrm{CH}_{2} \mathrm{Cl}_{2}$. The combined organic layers were dried over $\mathrm{Na}_{2} \mathrm{SO}_{4}$ and then concentrated in vacuo. The residue was applied to flash chromatography on silica gel $(\mathrm{PE}: \mathrm{EA}=10: 1)$ to afford product $\mathbf{G}(891.1 \mathrm{mg}, 76 \%)$ as a colorless oil. ${ }^{1} \mathrm{H}$ NMR (500 MHz, $\left.\mathrm{CDCl}_{3}\right): \delta=7.23-7.12(\mathrm{~m}, 4 \mathrm{H})$, $5.03(\mathrm{~s}, 0.97 \mathrm{H}), 3.50(\mathrm{q}, J=22.5 \mathrm{~Hz}, 2 \mathrm{H}), 2.84(\mathrm{~d}, J=16.0 \mathrm{~Hz}, 1 \mathrm{H}), 2.60(\mathrm{~d}, J=16.0 \mathrm{~Hz}, 1 \mathrm{H}), 2.08$ (s, 1H), 0.95 (s, 3H), $0.22(\mathrm{~s}, 9 \mathrm{H}) .{ }^{13} \mathrm{C} \mathrm{NMR}\left(125 \mathrm{~Hz}, \mathrm{CDCl}_{3}\right): \delta=144.1,140.3,127.4,126.3,124.8$, 
124.1, 78.0, 68.0, 50.0, 39.5, 17.1, 0.2. HRMS (ESI-TOF) $\mathrm{m} / \mathrm{z}:[\mathrm{M}+\mathrm{H}]^{+}$Calcd for $\mathrm{C}_{14} \mathrm{H}_{23} \mathrm{O}_{2} \mathrm{Si}$ 251.1462; Found 251.1463. FTIR (KBr, $\left.\mathrm{cm}^{-1}\right)$ : 3444.61, 3417.18, 3159.73, 3144.31, 1651.55, $1557.34,1455.32,1398.27$.

Dess-Martin periodinane (1.68 g, $3.97 \mathrm{~mol})$ was added to a solution of $\mathbf{G}(764.2 \mathrm{mg}, 3.05 \mathrm{~mol})$ in dry DCM $(26 \mathrm{~mL})$ at $0{ }^{\circ} \mathrm{C}$. The mixture was stirred at room temperature $3 \mathrm{~h}$ and quenched with water. The mixture was extracted with $\mathrm{CH}_{2} \mathrm{Cl}_{2}$. The combined organic layers were dried over $\mathrm{Na}_{2} \mathrm{SO}_{4}$ and concentrated in vacuo. The residue was applied to flash chromatography on silica gel (PE:EA $=100: 1)$ to afford product $7(628.1 \mathrm{mg}, 83 \%)$ as a colorless oil. ${ }^{1} \mathrm{H}$ NMR $\left(500 \mathrm{MHz}, \mathrm{CDCl}_{3}\right)$ : $\delta=9.71(\mathrm{~s}, 0.95 \mathrm{H}), 7.26-7.18(\mathrm{~m}, 4 \mathrm{H}), 5.40(\mathrm{~s}, 0.96 \mathrm{H}), 3.21(\mathrm{~d}, J=15.5 \mathrm{~Hz}, 1 \mathrm{H}), 2.74(\mathrm{~d}, J=16.0$ $\mathrm{Hz}, 1 \mathrm{H}), 1.18$ (s, 3H), 0.20 (s, 9H). ${ }^{13} \mathrm{C}$ NMR (125 Hz, $\left.\mathrm{CDCl}_{3}\right): \delta=204.3,143.1,138.8,128.2$, 127.1, 124.9, 124.4, 77.3, 60.6, 38.6, 14.6, 0.2. HRMS (ESI-TOF) m/z: $[\mathrm{M}+\mathrm{H}]^{+}$Calcd for $\mathrm{C}_{14} \mathrm{H}_{21} \mathrm{O}_{2} \mathrm{Si}$ 249.1305; Found 249.1305. FTIR (KBr, $\left.\mathrm{cm}^{-1}\right)$ : 3646.09, 3444.53, 3144.02, 1651.50, $1633.79,1557.26,1398.10$.<smiles>COC1=C(C=O)Cc2cc(-c3ccccc3)ccc21</smiles>

A screw-cap vial was charged with $\left[\mathrm{Ru}(p \text {-cymene }) \mathrm{Cl}_{2}\right]_{2}(5 \mathrm{~mol} \%, 0.005 \mathrm{mmol}, 3.1 \mathrm{mg})$, $\mathrm{AgSbF}_{6}(20 \mathrm{~mol} \%, 0.02 \mathrm{mmol}, 6.9 \mathrm{mg}), \mathrm{Cu}(\mathrm{OAc})_{2}(1.3$ equiv, $0.13 \mathrm{mmol}, 23.6 \mathrm{mg})$ and DCM (0.5 $\mathrm{mL})$. Then, aroylsilanes $\mathbf{1 n}(1.0$ equiv, $0.1 \mathrm{mmol}, 25.4 \mathrm{mg}), \mathbf{1 u}(1.0$ equiv, $0.1 \mathrm{mmol}, 24.0 \mathrm{mg})$ and 2a (3.0 equiv, $0.3 \mathrm{mmol}, 16.8 \mathrm{mg}$ ) were added into the solution in sequence. The vial was sealed under argon and heated to $60{ }^{\circ} \mathrm{C}$ in an oil bath with stirring for $16 \mathrm{~h}$. After cooling down, the mixture was directly applied to a flash column chromatography on silica gel (PE:EA = 100:1), and products 3na (18.8 mg, 30\%) and 3ua (11.7 mg, 20\%) were obtained. 
<smiles>COc1ccc(C(=O)c2ccc(Cl)cc2)cc1</smiles><smiles>C=CC=O</smiles><smiles>COC1=C(C=O)Cc2cc(Cl)ccc21</smiles>

3ka, 0\%<smiles>COc1ccc2c(c1)CC(C=O)=C2C</smiles>

A screw-cap vial was charged with $\left[\mathrm{Ru}(p \text {-cymene }) \mathrm{Cl}_{2}\right]_{2}(5 \mathrm{~mol} \%, 0.005 \mathrm{mmol}, 3.1 \mathrm{mg})$, $\mathrm{AgSbF}_{6}(20 \mathrm{~mol} \%, 0.02 \mathrm{mmol}, 6.9 \mathrm{mg}), \mathrm{Cu}(\mathrm{OAc})_{2}(1.3$ equiv, $0.13 \mathrm{mmol}, 23.6 \mathrm{mg})$ and $\mathrm{DCM}(0.5$ $\mathrm{mL})$. Then, aroylsilanes $\mathbf{1 k}$ (1.0 equiv, $0.1 \mathrm{mmol}, 21.2 \mathrm{mg})$, $\mathbf{1 h}(1.0$ equiv, $0.1 \mathrm{mmol}, 20.8 \mathrm{mg})$ and 2a (3.0 equiv, $0.3 \mathrm{mmol}, 16.8 \mathrm{mg}$ ) were added into the solution in sequence. The vial was sealed under argon and heated to $60^{\circ} \mathrm{C}$ in an oil bath with stirring for $1 \mathrm{~h}$. After cooling down, the mixture was directly applied to a flash column chromatography on silica gel (PE:EA $=70: 1)$, only leading to product $3 \mathrm{ha}(8.4 \mathrm{mg}, 32 \%)$.

\section{Deuterium Labeled Experiments}<smiles>[2H]c1c([2H])c([2H])c(C(=O)OC)c([2H])c1[2H]</smiles>
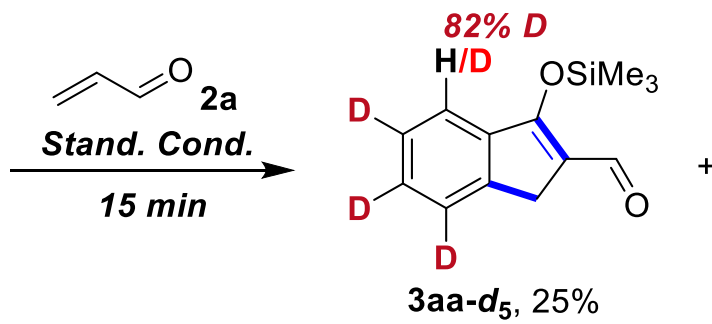<smiles>[2H]c1c([2H])c([2H])c(C(C)=O)c([2H])c1[2H]</smiles>

A screw-cap vial was charged with $\left[\mathrm{Ru}(p \text {-cymene }) \mathrm{Cl}_{2}\right]_{2}(5 \mathrm{~mol} \%, 0.005 \mathrm{mmol}), \mathrm{AgSbF}_{6}(20 \mathrm{~mol} \%$, $0.02 \mathrm{mmol}), \mathrm{Cu}(\mathrm{OAc})_{2}(1.3$ equiv, $0.13 \mathrm{mmol})$ and $\mathrm{DCM}(0.5 \mathrm{~mL})$. Then, 1a-d5 (1.0 equiv, 0.1 mmol) and 2a (3.0 equiv, $0.3 \mathrm{mmol}$ ) were added into the solution in sequence. The vial was sealed under argon and heated to $60{ }^{\circ} \mathrm{C}$ in an oil bath with stirring for $15 \mathrm{~min}$. After cooling down, the mixture was directly applied to a flash column chromatography on silica gel (3aa-d5 PE:EA = 80:1; 1a-d5 PE:EA = 200:1), affording 3aa-d5 (5.9 mg, 25\% yield) as a yellow solid and 1a-d5 (11.1 $\mathrm{mg}, 61 \%$ yield) as a yellow oil. The deuterium incorporation was determined by ${ }^{1} \mathrm{H}$ NMR. 

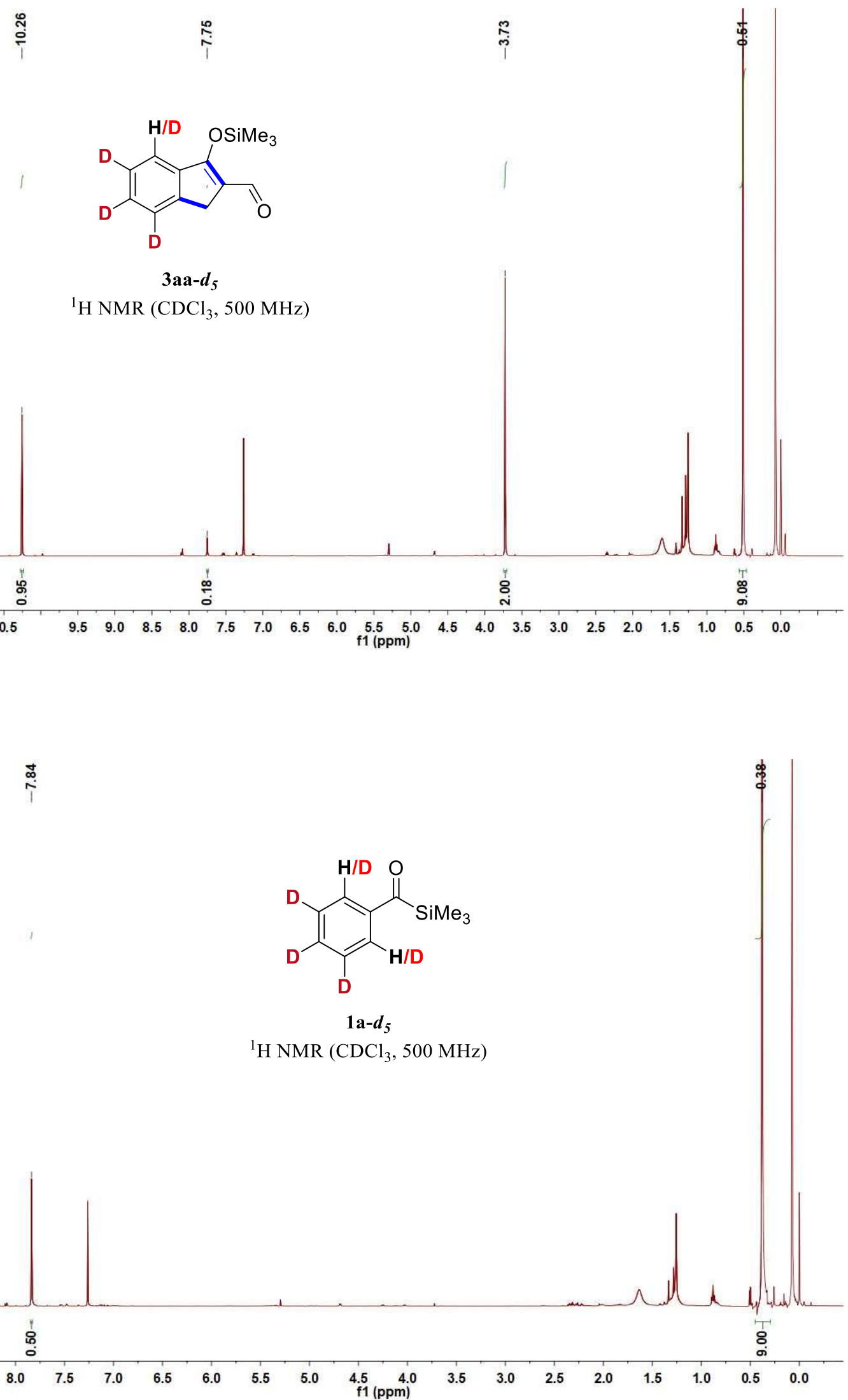

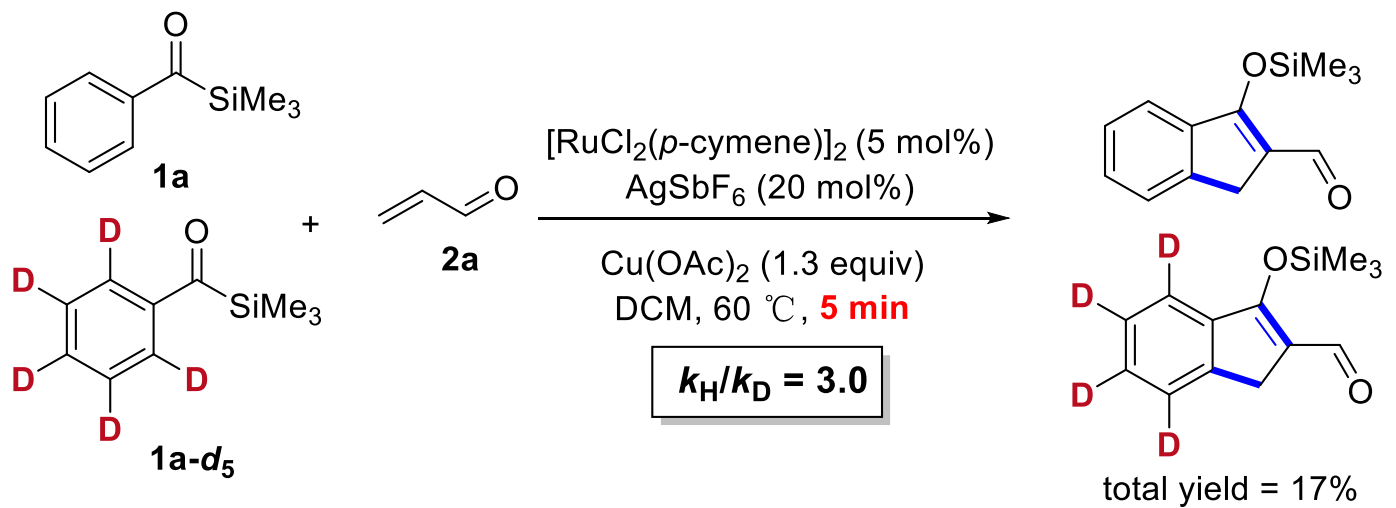

A screw-cap vial was charged with $\left[\mathrm{Ru}(p \text {-cymene }) \mathrm{Cl}_{2}\right]_{2}(5 \mathrm{~mol} \%, 0.01 \mathrm{mmol}), \mathrm{AgSbF}_{6}(20$ $\mathrm{mol} \%, 0.04 \mathrm{mmol}), \mathrm{Cu}(\mathrm{OAc})_{2}(1.3$ equiv, $0.26 \mathrm{mmol})$ and $\mathrm{DCM}(1.0 \mathrm{~mL})$. Then, $1 \mathrm{a}(1.0$ equiv, 0.1 mmol), 1a-d $\mathbf{5}$ (1.0 equiv, $0.1 \mathrm{mmol})$ and $\mathbf{2 a}(3.0$ equiv, $0.6 \mathrm{mmol})$ were added into the solution in sequence. The vial was sealed under argon and heated to $60{ }^{\circ} \mathrm{C}$ in an oil bath with stirring for $5 \mathrm{~min}$. After cooling down, the mixture was directly applied to a flash column chromatography on silica gel (PE:EA $=160: 1)$, affording the product as a light yellow solid $(7.9 \mathrm{mg}, 17 \%$ total yield $)$.

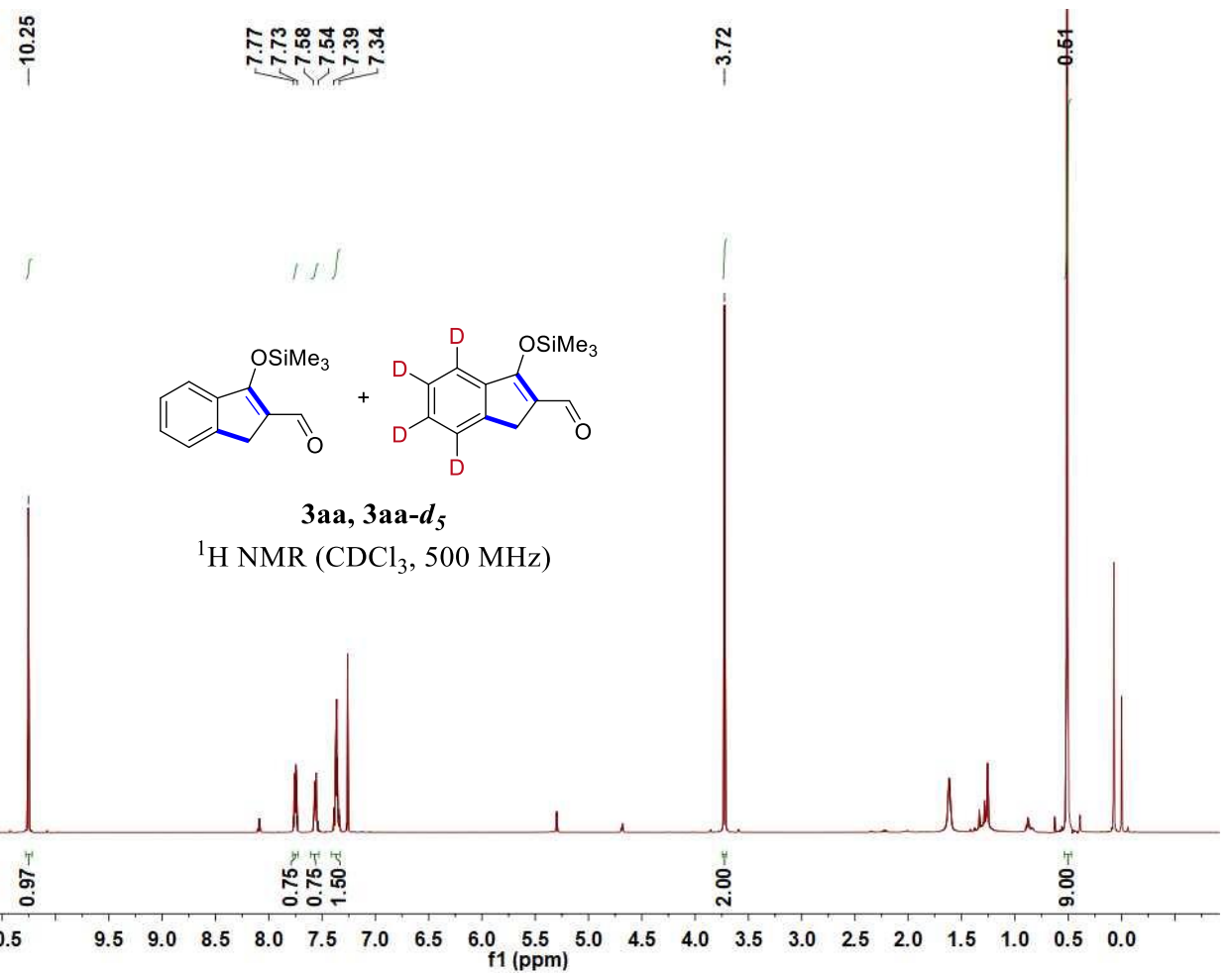




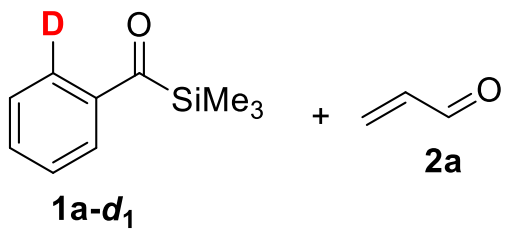

$$
\begin{gathered}
{\left[\mathrm{RuCl}_{2}(p \text {-cymene })\right]_{2}(5 \mathrm{~mol} \%)} \\
\operatorname{AgSbF}_{6}(20 \mathrm{~mol} \%)
\end{gathered}
$$

$$
k_{\mathrm{H}} / k_{\mathrm{D}}=1.2
$$<smiles>[2H]c1cccc2c1C(OC)=C(C=O)C2</smiles>

$3 \mathrm{aa}-d_{1}, 64 \%$

A screw-cap vial was charged with $\left[\mathrm{Ru}(p \text {-cymene }) \mathrm{Cl}_{2}\right]_{2}(5 \mathrm{~mol} \%, 0.01 \mathrm{mmol}), \mathrm{AgSbF}_{6}(20$ mol\%, $0.04 \mathrm{mmol}), \mathrm{Cu}(\mathrm{OAc})_{2}(1.3$ equiv, $0.26 \mathrm{mmol})$ and $\mathrm{DCM}(1.0 \mathrm{~mL})$. Then, 1 a-d $\mathbf{d}_{\mathbf{1}}(1.0$ equiv, $0.2 \mathrm{mmol}$ ) and $\mathbf{2 a}$ (3.0 equiv, $0.6 \mathrm{mmol})$ were added into the solution in sequence. The vial was sealed under argon and heated to $60{ }^{\circ} \mathrm{C}$ in an oil bath with stirring for $16 \mathrm{~h}$. After cooling down, the mixture was directly applied to a flash column chromatography on silica gel (PE:EA $=160: 1)$, affording the product 3aa-d $\mathbf{d}_{1}$ as a light yellow solid (30 $\mathrm{mg}, 64 \%$ yield).

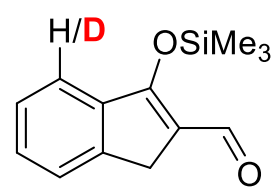

3aa-d 1

${ }^{1} \mathrm{H}$ NMR $\left(\mathrm{CDCl}_{3}, 500 \mathrm{MHz}\right)$

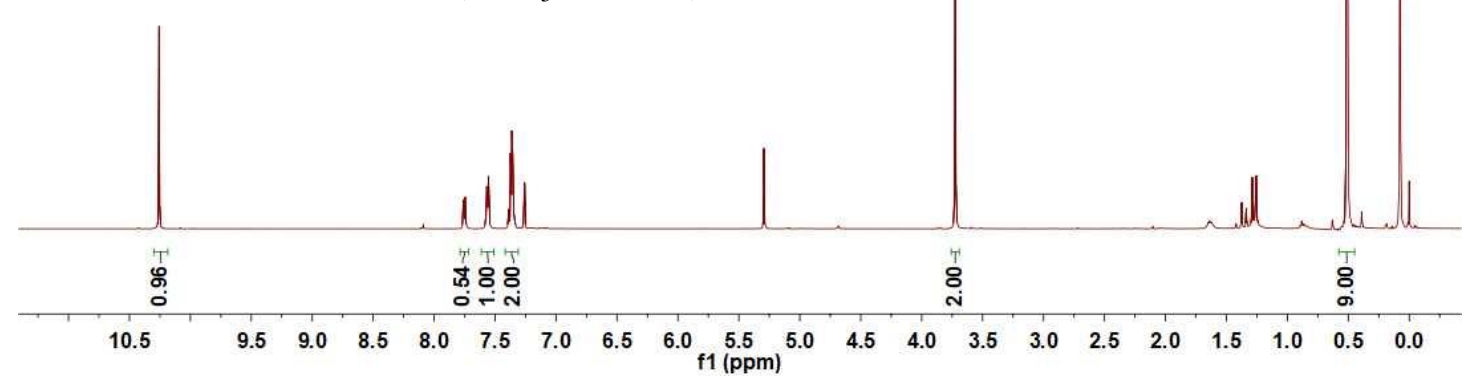

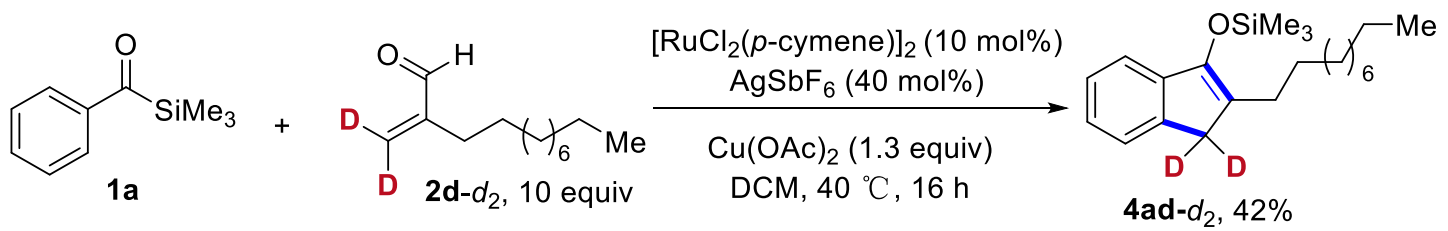

A screw-cap vial was charged with $\left[\mathrm{Ru}(p \text {-cymene }) \mathrm{Cl}_{2}\right]_{2}(10 \mathrm{~mol} \%, 0.01 \mathrm{mmol}), \mathrm{AgSbF}_{6}(40$ 
$\mathrm{mol} \%, 0.04 \mathrm{mmol}), \mathrm{Cu}(\mathrm{OAc})_{2}(1.3$ equiv, $0.13 \mathrm{mmol})$ and $\mathrm{DCM}(0.5 \mathrm{~mL})$. Then, $1 \mathrm{a}(17.8 \mathrm{mg}, 0.1$ mmol) and $\mathbf{2 d - d _ { 2 }}$ (10.0 equiv, $\left.1.0 \mathrm{mmol}\right)$ were added into the solution in sequence. The vial was sealed under argon and heated to $40{ }^{\circ} \mathrm{C}$ in an oil bath with stirring for $16 \mathrm{~h}$. After cooling down, the mixture was directly applied to a flash column chromatography on silica gel (PE), affording $\mathbf{4 a d}-\mathbf{d}_{2}$ as a light yellow oil ( $14.5 \mathrm{mg}, 42 \%$ yield).

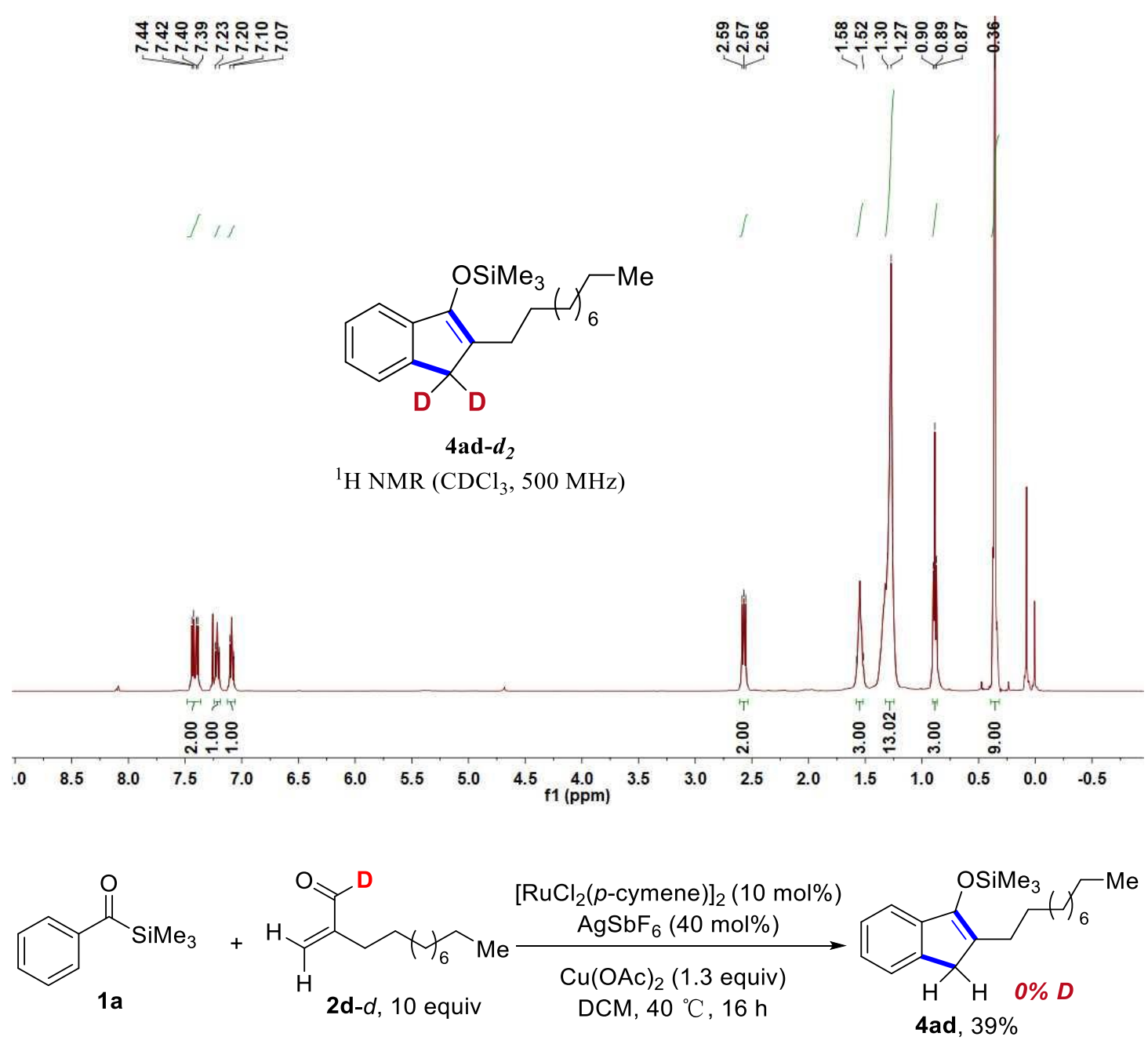

A screw-cap vial was charged with $\left[\mathrm{Ru}(p \text {-cymene }) \mathrm{Cl}_{2}\right]_{2}(10 \mathrm{~mol} \%, 0.01 \mathrm{mmol}), \mathrm{AgSbF}_{6}(40 \mathrm{~mol} \%$, $0.04 \mathrm{mmol}), \mathrm{Cu}(\mathrm{OAc})_{2}(1.3$ equiv, $0.13 \mathrm{mmol})$ and $\mathrm{DCM}(0.5 \mathrm{~mL})$. Then, $1 \mathrm{a}(17.8 \mathrm{mg}, 0.1 \mathrm{mmol})$ and $\mathbf{2 d - d _ { 2 }}$ (10.0 equiv, $1.0 \mathrm{mmol}$ ) were added into the solution in sequence. The vial was sealed under argon and heated to $40{ }^{\circ} \mathrm{C}$ in an oil bath with stirring for $16 \mathrm{~h}$. After cooling down, the mixture was directly applied to a flash column chromatography using PE as the eluent, affording 4ad as a yellow oil (13.4 mg, 39\% yield). 


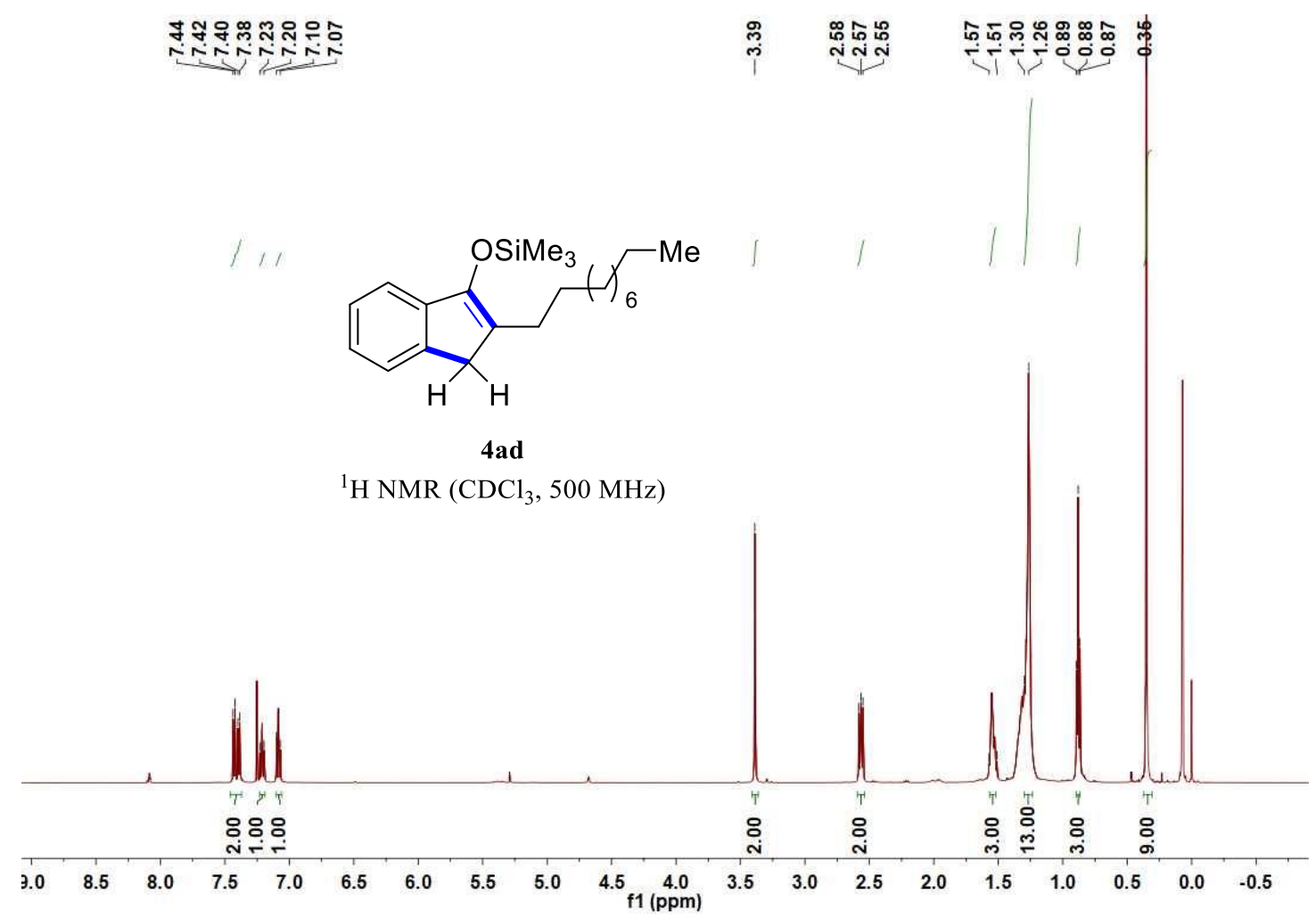

\section{Application Development}<smiles>COC1=C(C=O)Cc2ccccc21</smiles>

3aa<smiles>NNc1ccccc1</smiles>

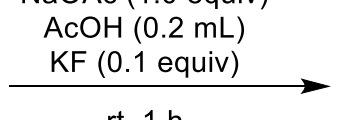

rt. $1 \mathrm{~h}$

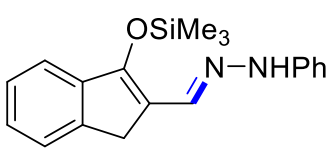

$8,99 \%$

A screw-cap vial was charged with $\mathrm{NaOAc}$ (1.0 equiv, $0.1 \mathrm{mmol}), \mathrm{AcOH}(0.2 \mathrm{~mL}), \mathrm{KF}$ (0.1 equiv, $0.01 \mathrm{mmol})$. Then 3 aa $(23.2 \mathrm{mg}, 0.1 \mathrm{mmol})$ and $\mathrm{PhNHNH}_{2}(1.1$ equiv, 0.11 mmol) were added in sequence. The vial was sealed under argon and stirring in room te mperature for $1 \mathrm{~h}$. The mixture was quenched with saturated aqueous $\mathrm{NaHCO}_{3}$ to $\mathrm{pH} 8$. After the mixture was allowed to warm to ambient temperature, then taken up in EtOAc and washed three times with brine. The combined organic layers were dried over $\mathrm{Na}_{2} \mathrm{SO}_{4}$, then concentrated in vacuo. Compound $\mathbf{8}$ was obtained as a brown oil $(31.9 \mathrm{mg}$, yield $=$ 99\%) by flash chromatography on silica gel $(\mathrm{PE}: \mathrm{EA}=200: 1) .{ }^{1} \mathrm{H}$ NMR $(500 \mathrm{MHz}, \mathrm{CDCl}$ 3): $\delta=7.98(\mathrm{~s}, 1 \mathrm{H}), 7.64(\mathrm{~s}, 1 \mathrm{H}), 7.57(\mathrm{~d}, J=7.5 \mathrm{~Hz}, 1 \mathrm{H}), 7.50(\mathrm{~d}, J=7.0 \mathrm{~Hz}, 1 \mathrm{H})$, 7.34-7.28 (m, 3H), 7.23-7.20 (td, $J=7.5 \mathrm{~Hz}, J=1.0 \mathrm{~Hz}, 1 \mathrm{H}), 7.14-7.12(\mathrm{~m}, 2 \mathrm{H}), 6.94-6$. 
$91(\mathrm{~m}, 1 \mathrm{H}), 3.94(\mathrm{~s}, 2 \mathrm{H}), 0.49(\mathrm{~s}, 9 \mathrm{H}) .{ }^{13} \mathrm{C} \mathrm{NMR}\left(125 \mathrm{~Hz}, \mathrm{CDCl}_{3}\right): \delta=152.2,148.2,143$. $5,142.4,141.2,134.8,125.2,124.0,122.7,121.5,119.3,111.9,39.4,0.2$. HRMS (ESI) $\mathrm{m}$ lz: $[\mathrm{M}+\mathrm{H}]^{+}$Calcd for $\mathrm{C}_{19} \mathrm{H}_{23} \mathrm{~N}_{2} \mathrm{OSi}$ 323.1574; Found 323.1551. FTIR $\left(\mathrm{KBr}, \mathrm{cm}^{-1}\right): 3451$. $80,3416.80,2954.62,2922.40,1642.85,1602.16,1503.41,1401.52,1251.64$.

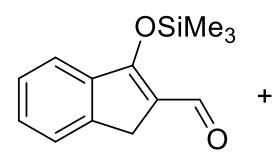

$3 a a$

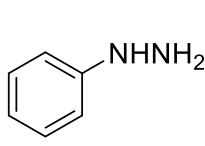

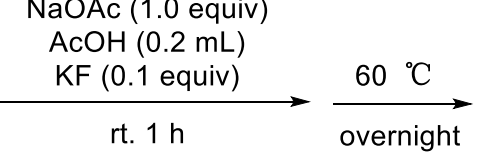

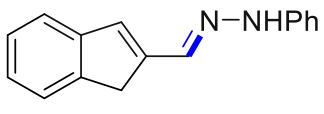

9, $91 \%$

A screw-cap vial was charged with $\mathrm{NaOAc}(1.0$ equiv, $0.1 \mathrm{mmol}), \mathrm{AcOH}(0.2 \mathrm{~mL}), \mathrm{F}$ $\mathrm{K}$ (0.1 equiv , $0.01 \mathrm{mmol})$. Then 3aa $(23.2 \mathrm{mg}, 0.1 \mathrm{mmol})$ and $\mathrm{PhNHNH}_{2}(1.1$ equiv, 0.1 $1 \mathrm{mmol}$ ) were added in sequence. The vial was sealed under argon and stirring in room $\mathrm{t}$ emperature for $1 \mathrm{~h}$, then heated to $60{ }^{\circ} \mathrm{C}$ in an oil bath with stirring overnight. After th e mixture was allowed to warm to ambient temperature, the mixture was quenched with $\mathrm{s}$ aturated aqueous $\mathrm{NaHCO}_{3}$ to $\mathrm{pH}$ 8, then taken up in EtOAc and washed three times with brine. The combined organic layers were dried over $\mathrm{Na}_{2} \mathrm{SO}_{4}$, then concentrated in vacuo. Compound 9 as obtained a brown oil $(21.3 \mathrm{mg}$, yield $=91 \%)$ by flash chromatography o n silica gel $(\mathrm{PE}: \mathrm{EA}=15: 1) .{ }^{1} \mathrm{H}$ NMR $\left(500 \mathrm{MHz}, \mathrm{CDCl}_{3}\right): \delta=7.75(\mathrm{~s}, 1 \mathrm{H}), 7.58(\mathrm{~s}, 1 \mathrm{H})$, $7.49(\mathrm{~d}, J=7.0 \mathrm{~Hz}, 1 \mathrm{H}), 7.39(\mathrm{~d}, J=7.5 \mathrm{~Hz}, 1 \mathrm{H}), 7.33-7.28(\mathrm{~m}, 3 \mathrm{H}), 7.23(\mathrm{t}, J=7.0$ $\mathrm{Hz}, 1 \mathrm{H}), 7.12(\mathrm{~d}, J=8.5 \mathrm{~Hz}, 2 \mathrm{H}), 6.93-6.89(\mathrm{~m}, 2 \mathrm{H}), 3.83(\mathrm{~s}, 2 \mathrm{H}) .{ }^{13} \mathrm{C}$ NMR $(125 \mathrm{H}$ $\left.\mathrm{z}, \mathrm{CDCl}_{3}\right): \delta=144.7,144.6,144.5,143.3,135.2,131.6,129.4,126.6,125.4,123.9,121.2$, 120.2, 112.8. HRMS (ESI) m/z: $[\mathrm{M}+\mathrm{H}]^{+}$Calcd for $\mathrm{C}_{16} \mathrm{H}_{15} \mathrm{~N}_{2}$ 235.1230; Found 235.1234. FTIR $\left(\mathrm{KBr}, \mathrm{cm}^{-1}\right):$ 3454.68, 3422.99, 3093.24, 2921.38, 2850.15, 1651.17, 1633.00, 1402. 54, 987.09 .<smiles>COC1=C(C)Cc2ccccc21</smiles>

$4 a b$

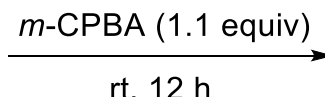

rt. $12 \mathrm{~h}$

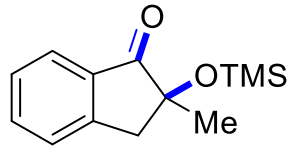

$11,83 \%$ 
Compound 4ab $(21.8 \mathrm{mg}, 0.1 \mathrm{mmol})$ was dissolved in $\mathrm{CH}_{2} \mathrm{Cl}_{2}(0.5 \mathrm{~mL})$ and $m$-CPBA (0.11 mmol) ware added at $0{ }^{\circ} \mathrm{C}$. After warming up to $\mathrm{rt}$ the mixture was stirred for 12 h. The mixture was allowed to warm to ambient temperature, then taken up in $\mathrm{CH}_{2} \mathrm{Cl}_{2}$ an d washed three times with brine. The combined organic layers were dried over $\mathrm{Na}_{2} \mathrm{SO}_{4}$, th en concentrated in vacuo. Compound $\mathbf{1 1}$ was obtained as a colorless oil $(19.4 \mathrm{mg}$, yield = $83 \%$ ) by flash chromatography on silica gel using PE as the eluent. ${ }^{1} \mathrm{HNMR}$ (500 MHz, $\left.\mathrm{CDCl}_{3}\right): \delta=7.72(\mathrm{~d}, J=7.5 \mathrm{~Hz}, 1 \mathrm{H}), 7.56-7.53(\mathrm{td}, J=7.5 \mathrm{~Hz}, J=1.0 \mathrm{~Hz}, 1 \mathrm{H}), 7.42$ $(\mathrm{d}, J=7.5 \mathrm{~Hz}, 1 \mathrm{H}), 7.35(\mathrm{t}, J=8.0 \mathrm{~Hz}, 1 \mathrm{H}), 3.29(\mathrm{~d}, J=18.0 \mathrm{~Hz}, 1 \mathrm{H}), 2.86(\mathrm{~d}, J=1$ $7.5 \mathrm{~Hz}, 1 \mathrm{H}), 1.39(\mathrm{~s}, 3 \mathrm{H}),-0.05(\mathrm{~s}, 9 \mathrm{H}) .{ }^{13} \mathrm{C} \mathrm{NMR}\left(125 \mathrm{~Hz}, \mathrm{CDCl}_{3}\right): \delta=214.8,156.7,14$ 2.5, 138.3, 131.7, 130.2, 127.8, 48.8, 42.9, 22.8, 0.2. HRMS (ESI) m/z: $[\mathrm{M}+\mathrm{H}]^{+}$Calcd for $\mathrm{C}_{13} \mathrm{H}_{19} \mathrm{O}_{2} \mathrm{Si}$ 235.1149; Found 235.1146. FTIR $\left(\mathrm{KBr}, \mathrm{cm}^{-1}\right)$ : 3447.89, 3423.57, 2945.62, 2796.97, 1734.19, 1657.89, 897.63.<smiles>COC1=C(C=O)Cc2ccccc21</smiles>

3aa

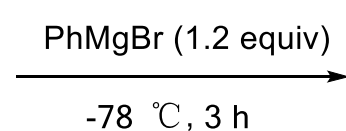

$-78{ }^{\circ} \mathrm{C}, 3 \mathrm{~h}$

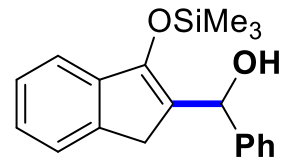

$10,83 \%$

$\operatorname{PhMgBr}(1 \mathrm{M}$ in THF, $0.12 \mathrm{mmol})$ was dissolved in THF $(0.25 \mathrm{~mL})$ and 3aa $(23.2$ $\mathrm{mg}, 0.1 \mathrm{mmol})$ was dissolved in THF $(0.25 \mathrm{~mL})$ ware added at $0{ }^{\circ} \mathrm{C}$. After warming up $\mathrm{t}$ $\mathrm{o}$ rt the mixture was stirred for $3 \mathrm{~h}$. The mixture was quenched with saturated aqueous $\mathrm{N}$ $\mathrm{H}_{4} \mathrm{Cl}$. After the mixture was allowed to warm to ambient temperature, then taken up in Et OAc and washed three times with brine. The combined organic layers were dried over $\mathrm{Na}$ ${ }_{2} \mathrm{SO}_{4}$ and concentrated in vacuo. Compound $\mathbf{1 0}$ was obtained as a yellow oil (25.8 $\mathrm{mg}$, yi eld $=83 \%)$ by flash chromatography on silica gel $(\mathrm{PE}: \mathrm{EA}=50: 1) .{ }^{1} \mathrm{H}$ NMR $(500 \mathrm{MHz}$, $\left.\mathrm{CDCl}_{3}\right): \delta=7.53(\mathrm{~d}, J=8.0 \mathrm{~Hz}, 1 \mathrm{H}), 7.40(\mathrm{~d}, J=7.0 \mathrm{~Hz}, 2 \mathrm{H}), 7.36-7.30(\mathrm{~m}, 3 \mathrm{H}), 7.2$ 6-7.22 (m, 2H), 7.14-7.11 (m, 1H), $6.10(\mathrm{~s}, 1 \mathrm{H}), 3.60(\mathrm{~d}, J=23.5 \mathrm{~Hz}, 1 \mathrm{H}), 3.10(\mathrm{~d}, J=$ $23.5 \mathrm{~Hz}, 1 \mathrm{H}), 0.45(\mathrm{~s}, 9 \mathrm{H}) .{ }^{13} \mathrm{C} \mathrm{NMR}\left(125 \mathrm{~Hz}, \mathrm{CDCl}_{3}\right): \delta=158.8,147.7,143.0,142.3,1$ 39.1, 127.6, 126.5, 125.3, 124.9, 123.7, 123,0, 121.9, 70.6, 38.1, 0.2. HRMS (ESI) m/z: $[\mathrm{M}+\mathrm{H}]^{+}$Calcd for $\mathrm{C}_{19} \mathrm{H}_{23} \mathrm{O}_{2} \mathrm{Si}$ 311.1462; Found 311.1460. FTIR $\left(\mathrm{KBr}, \mathrm{cm}^{-1}\right): 3451.61,3$ 
421.72, 2966.31, 2359.56, 2333.63, 1658.81, 1632.69, 1402.41.

\section{Gram-scale synthesis}

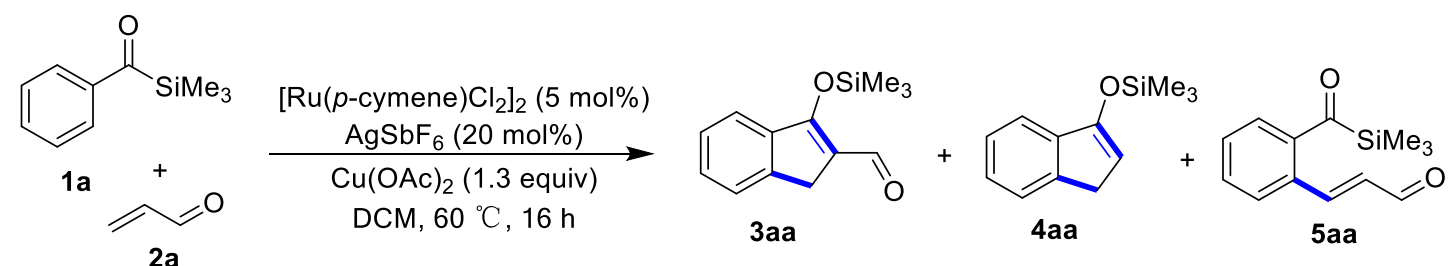

A screw-cap vial was charged with $\left[\mathrm{Ru}(p \text {-cymene }) \mathrm{Cl}_{2}\right]_{2}(5 \mathrm{~mol} \%, 0.3 \mathrm{mmol}, 0.18 \mathrm{~g}), \mathrm{AgSbF}_{6}(20$ mol\%, $1.2 \mathrm{mmol}, 0.41 \mathrm{~g}), \mathrm{Cu}(\mathrm{OAc})_{2}(1.3$ equiv, $7.8 \mathrm{mmol}, 1.42 \mathrm{~g})$ and $\mathrm{DCM}(30 \mathrm{~mL})$. Then, aroylsilane 1 (1.0 equiv, $6.0 \mathrm{mmol}, 1.07 \mathrm{~g})$ and acrolein $2(3.0$ equiv, $18.0 \mathrm{mmol}, 1.01 \mathrm{~g})$ were added into the solution in sequence. The vial was sealed under argon and heated to $60{ }^{\circ} \mathrm{C}$ in an oil bath with stirring for $16 \mathrm{~h}$. After cooling down, the mixture was directly applied to a flash column chromatography on silica gel for separation (3aa, PE:EA = 160:1; 4aa, PE; 5aa, PE:EA = 60:1). (3aa, $780.6 \mathrm{mg}$, yield $=42 \%$; 4aa, $41.8 \mathrm{mg}$, yield $=3 \%$; 5aa, $34.0 \mathrm{mg}$, yield $=2 \%$ ). 


\section{References}

1. A. Hayashi, S. Suzuki, J. Tsuji, K. Yamamoto, Organometallics, 1987, 6, 974.

2. B. F. Bonini, M. C. Franchini, M. Fochi, J. Chem. Soc., Perkin Trans. 1995, 1, 2039.

3. Z. P. Zhang, Z. Y. Han, G. X. Gu, Adv. Synth. Catal. 2017, 359, 2585.

4. P. Becker, D. L. Priebbenow, R. Pirwerdjan, C. Bolm, Angew. Chem. Int. Ed. 2014, 53, 269.

5. T. Kashiwabara, M. Tanaka, Organometallics, 2006, 25, 4648.

6. J. R. Huckins, S. D. Rychnovsky, J. Org. Chem., 2003, 68, 10135.

7. X. F. Wu, H. Neumann, M. Beller, Tetrahedron Lett., 2012, 53, 582.

8. A. F. Patrocinio, Paulo. J. S. Moran, J. Organometallic Chem., 2000, 603, 220.

9. X. Lu, C. Shen, J. Zhang, G. Zhong, Chem. Commun., 2019, 55, 826-829.

10. Z. Zhang, Z. Han, X.-Q. Dong, X. Zhang, Adv. Synth. Catal., 2017, 15, 2585-2589.

11. O. Knopff, A. Alexakis, Org. Lett., 2002, 4, 3835-3837.

12. Y. Dai, X. Q. Feng, H. F. Du, Org. Lett., 2019, 21, 6884-6887.

13. P. A. Marshall, R. H. Prager, Aust. J. Chem., 1979, 32, 1251-1260.

14. T. Mahapatra, N. Jana, S. Nanda, Tetrahedron: Asymmetry, 2008, 19, 1224-1232. 
NMR Spectra

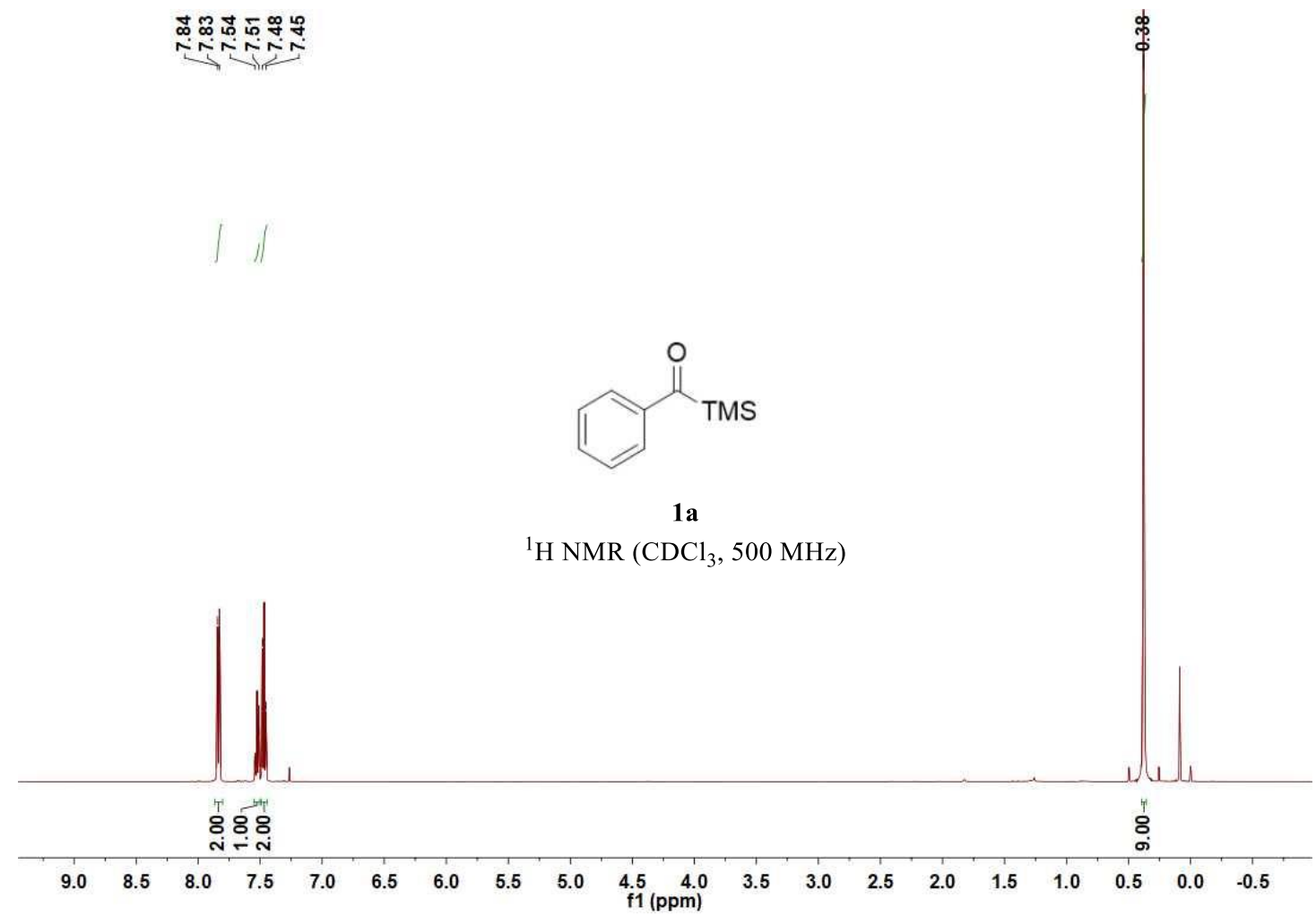

กิ ฺุ

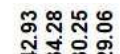

ปูํำ

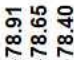

กุ

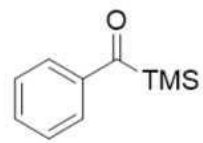

$1 \mathrm{a}$

${ }^{13} \mathrm{C} \mathrm{NMR}\left(\mathrm{CDCl}_{3}, 125 \mathrm{MHz}\right)$
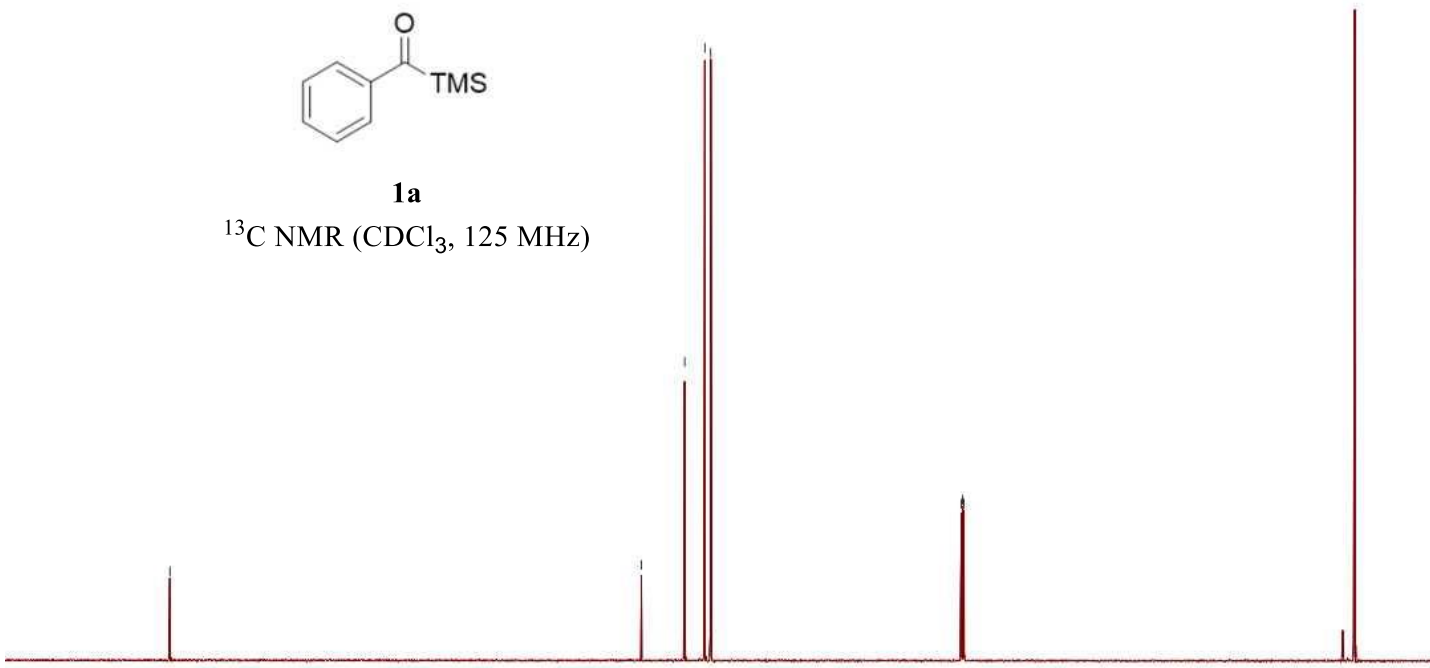

$\begin{array}{lllllllllllllllllllllll} & 250 & 230 & 210 & 190 & 170 & 150 & 130 & 110 & 90 & 80 & 70 & 60 & 50 & 40 & 30 & 20 & 10 & 0 & \end{array}$ 


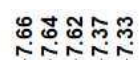

证侪

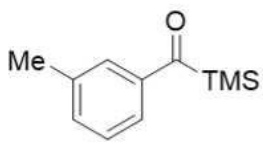

$1 \mathbf{b}$

${ }^{1} \mathrm{H}$ NMR $\left(\mathrm{CDCl}_{3}, 500 \mathrm{MHz}\right)$

豙

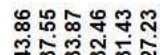

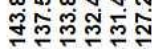

두용

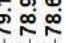

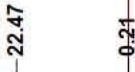

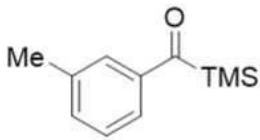

$1 \mathbf{b}$

${ }^{13} \mathrm{C} \mathrm{NMR}\left(\mathrm{CDCl}_{3}, 125 \mathrm{MHz}\right)$

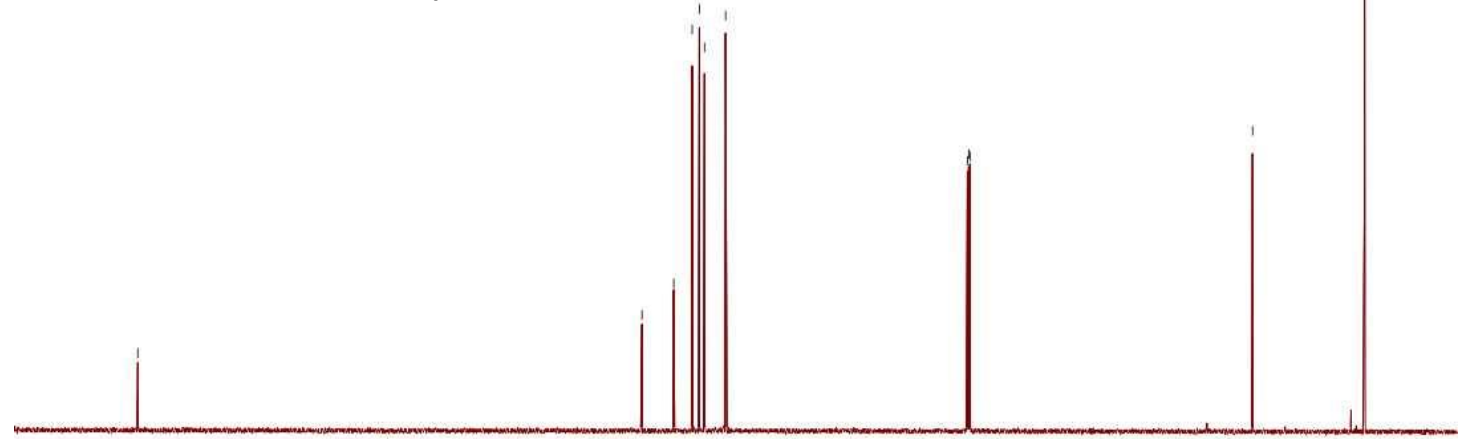

$250 \quad 230$

$\begin{array}{llll}190 & 170 & 150 & \begin{array}{l}130 \\ \mathrm{f} 1(\mathrm{ppm})\end{array}\end{array}$

$\begin{array}{llllllllll}90 & 80 & 70 & 60 & 50 & 40 & 30 & 20 & 10 & 0\end{array}$ 


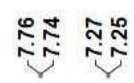

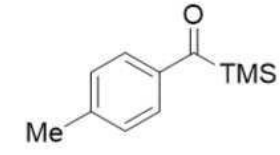

$1 \mathrm{c}$

${ }^{1} \mathrm{H}$ NMR $\left(\mathrm{CDCl}_{3}, 500 \mathrm{MHz}\right)$

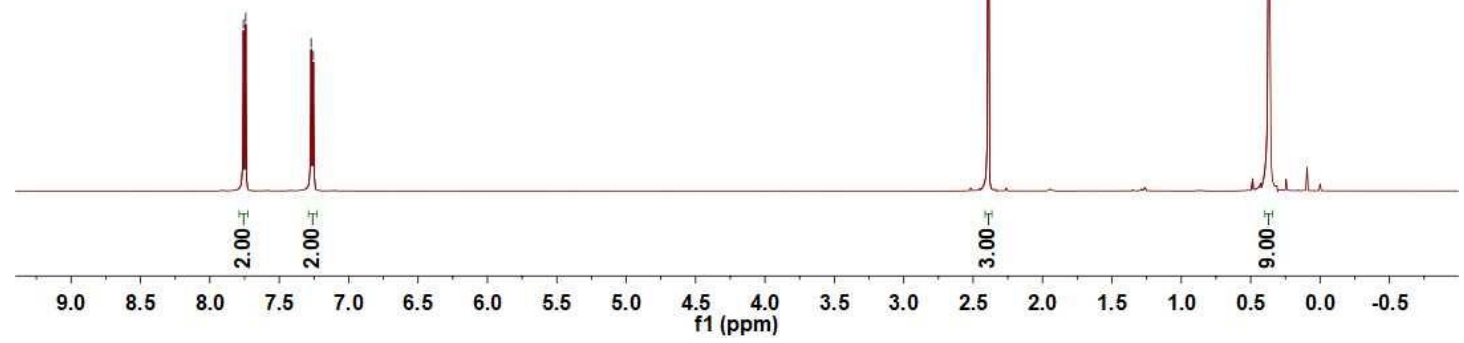

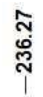

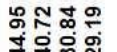

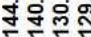

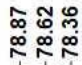

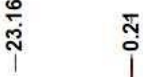

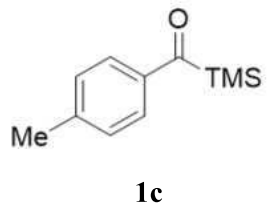

MS

${ }^{13} \mathrm{C} \mathrm{NMR}\left(\mathrm{CDCl}_{3}, 125 \mathrm{MHz}\right)$

$250 \quad 230$

210

$170 \quad 150$

130 f1 (ppm) 110

$\begin{array}{llllllllll}90 & 80 & 70 & 60 & 50 & 40 & 30 & 20 & 10 & 0\end{array}$ 


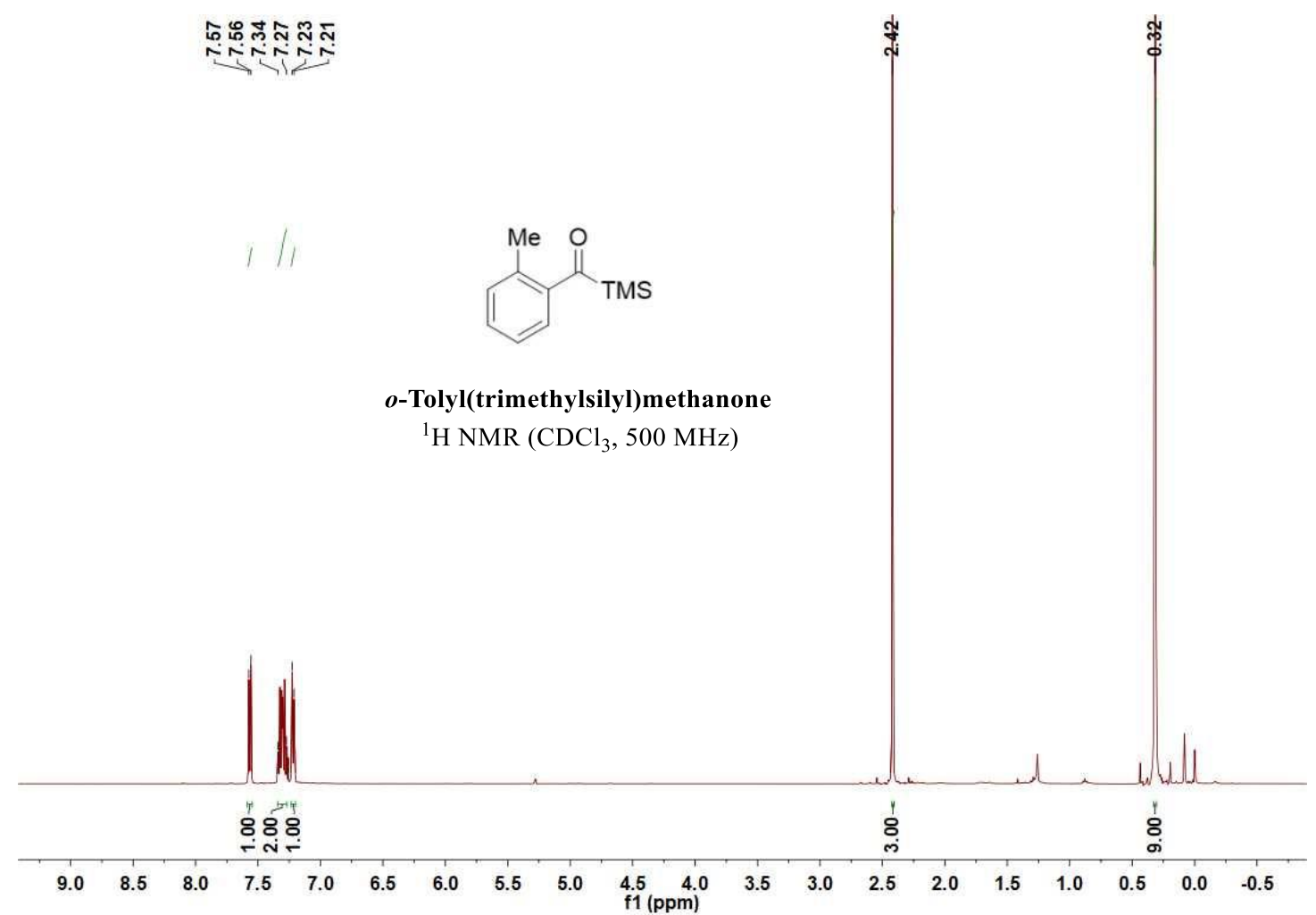

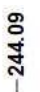
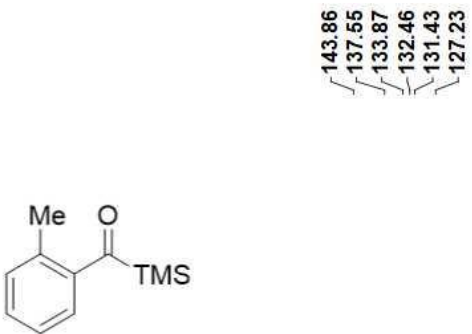

$o$-Tolyl(trimethylsilyl)methanone

${ }^{13} \mathrm{C} \mathrm{NMR}\left(\mathrm{CDCl}_{3}, 125 \mathrm{MHz}\right)$

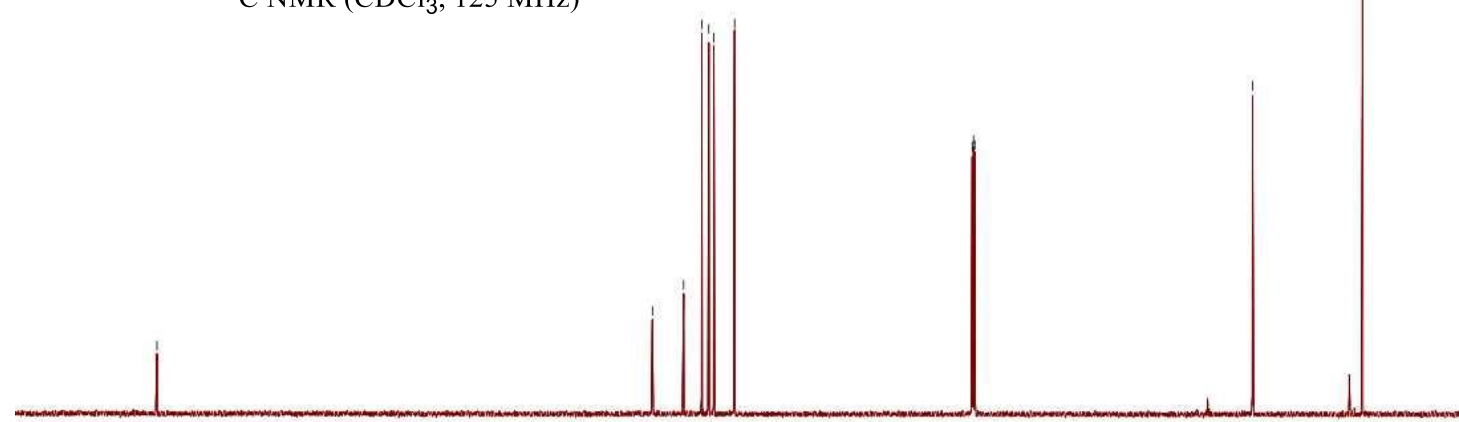

$\begin{array}{llllllllllllllllllll}170 & 250 & 230 & 210 & 190 & 170 & 150 & \begin{array}{c}130 \\ \mathrm{f} 1(\mathrm{ppm})\end{array} & 110 & 90 & 80 & 70 & 60 & 50 & 40 & 30 & 20 & 10 & 0 & \end{array}$ 
许

กูกตัติ

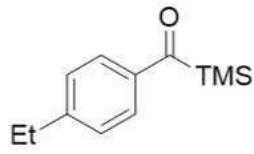

1d

${ }^{1} \mathrm{H} \mathrm{NMR}\left(\mathrm{CDCl}_{3}, 500 \mathrm{MHz}\right)$

ஸ্ं

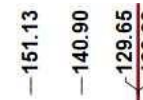

œొ

$\stackrel{\infty}{\wedge}$

?ำ

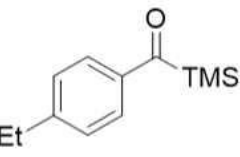

$1 d$

${ }^{13} \mathrm{C} \mathrm{NMR}\left(\mathrm{CDCl}_{3}, 125 \mathrm{MHz}\right)$

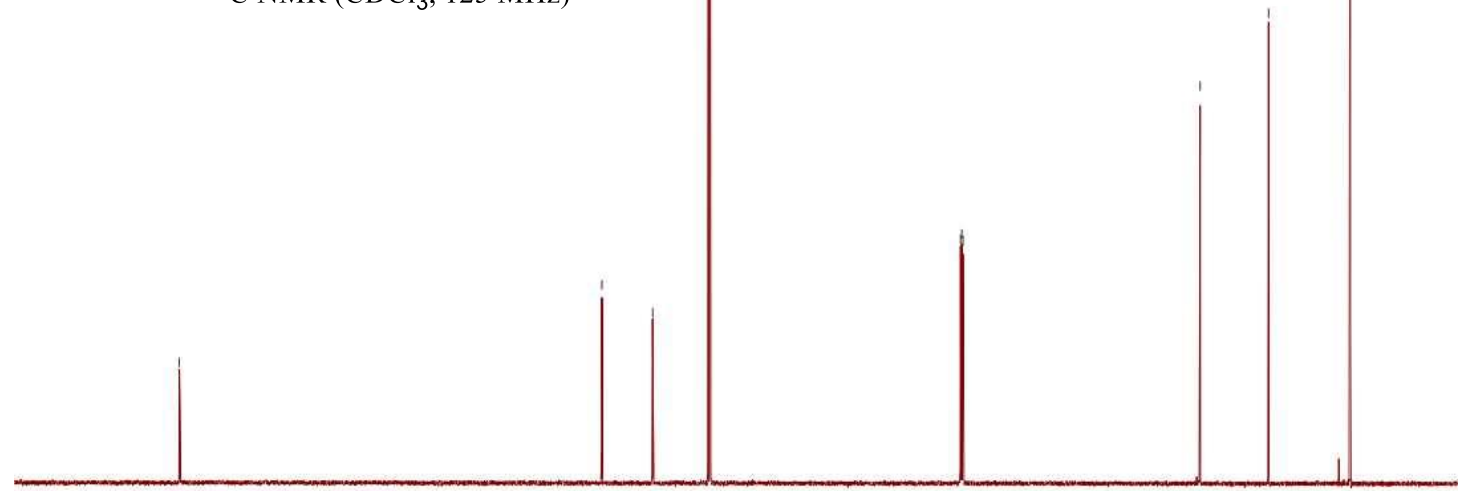

$250230 \quad 210 \quad 190 \quad 170 \quad 150 \quad \begin{array}{lllllllllllllll}130 & 110 & 90 & 80 & 70 & 60 & 50 & 40 & 30 & 20 & 10 & 0 & -10\end{array}$ 
令 䟥

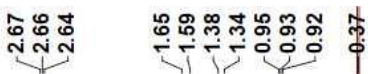

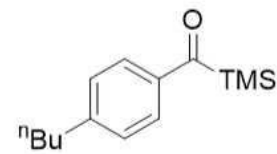

$1 \mathrm{e}$

${ }^{1} \mathrm{H} \mathrm{NMR}\left(\mathrm{CDCl}_{3}, 500 \mathrm{MHz}\right)$

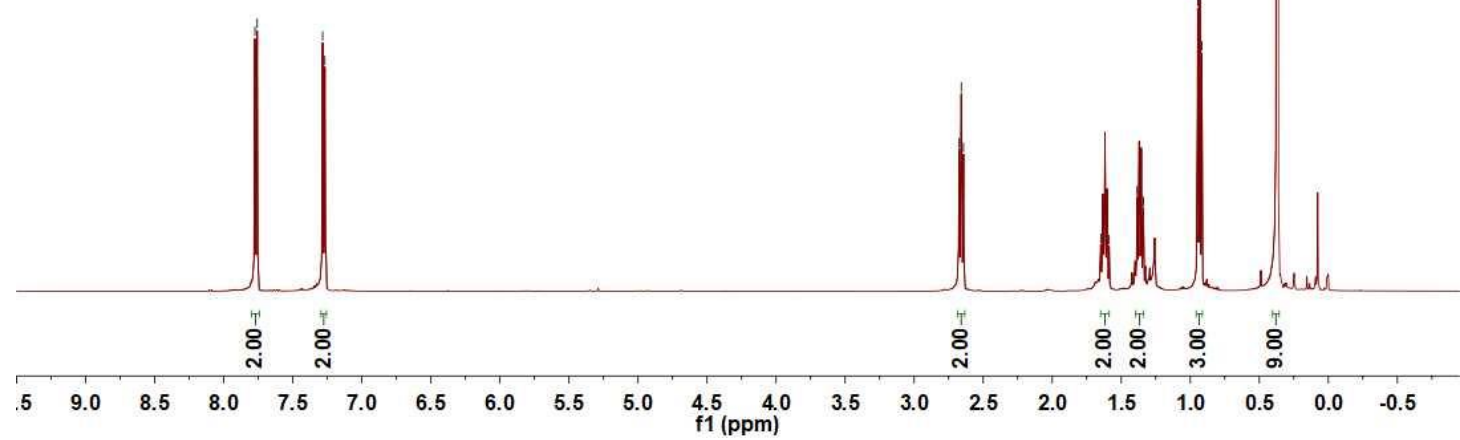

స్.

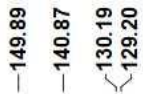

象

虫

สุ่

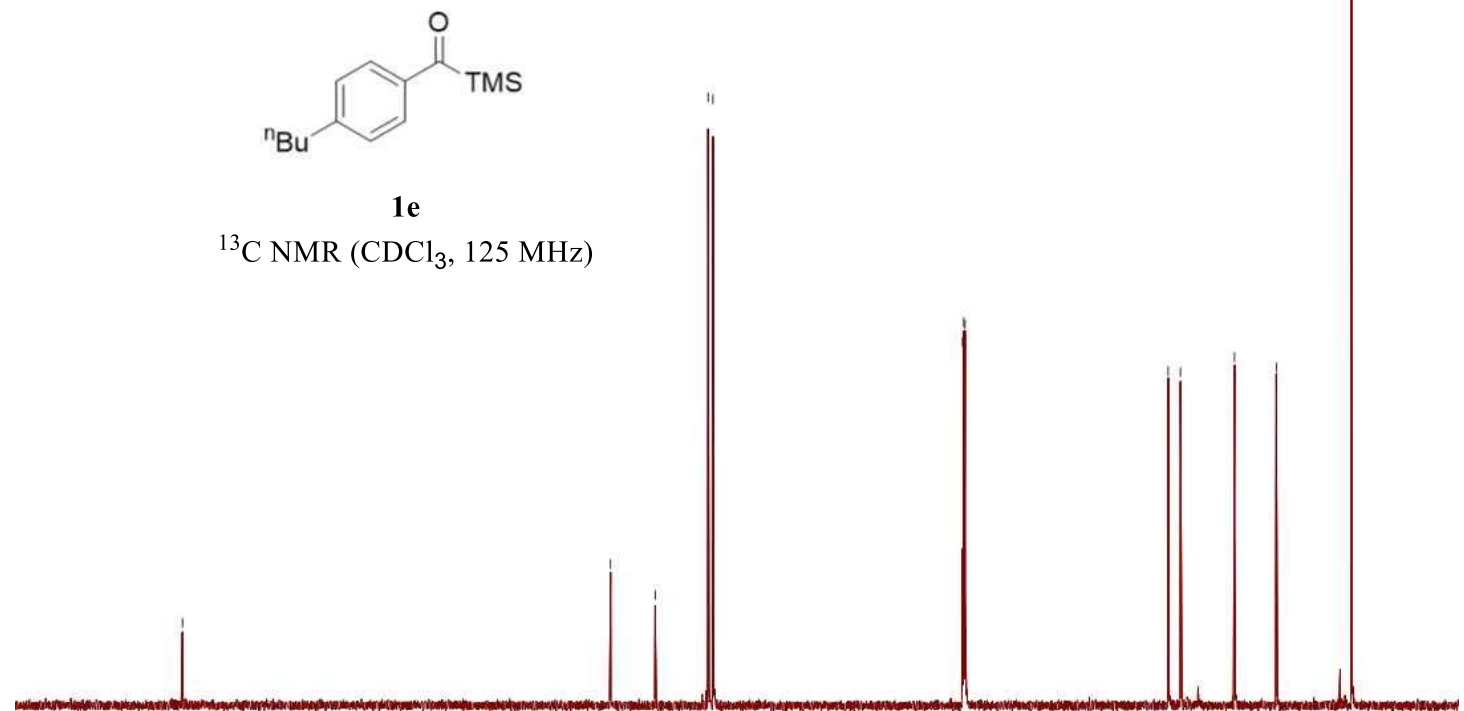

\begin{tabular}{lllllllllllllllllllll}
\hline 0 & 250 & 230 & 210 & 190 & 170 & 150 & 130 & 110 & 90 & 80 & 70 & 60 & 50 & 40 & 30 & 20 & 10 & 0 & -10
\end{tabular} 

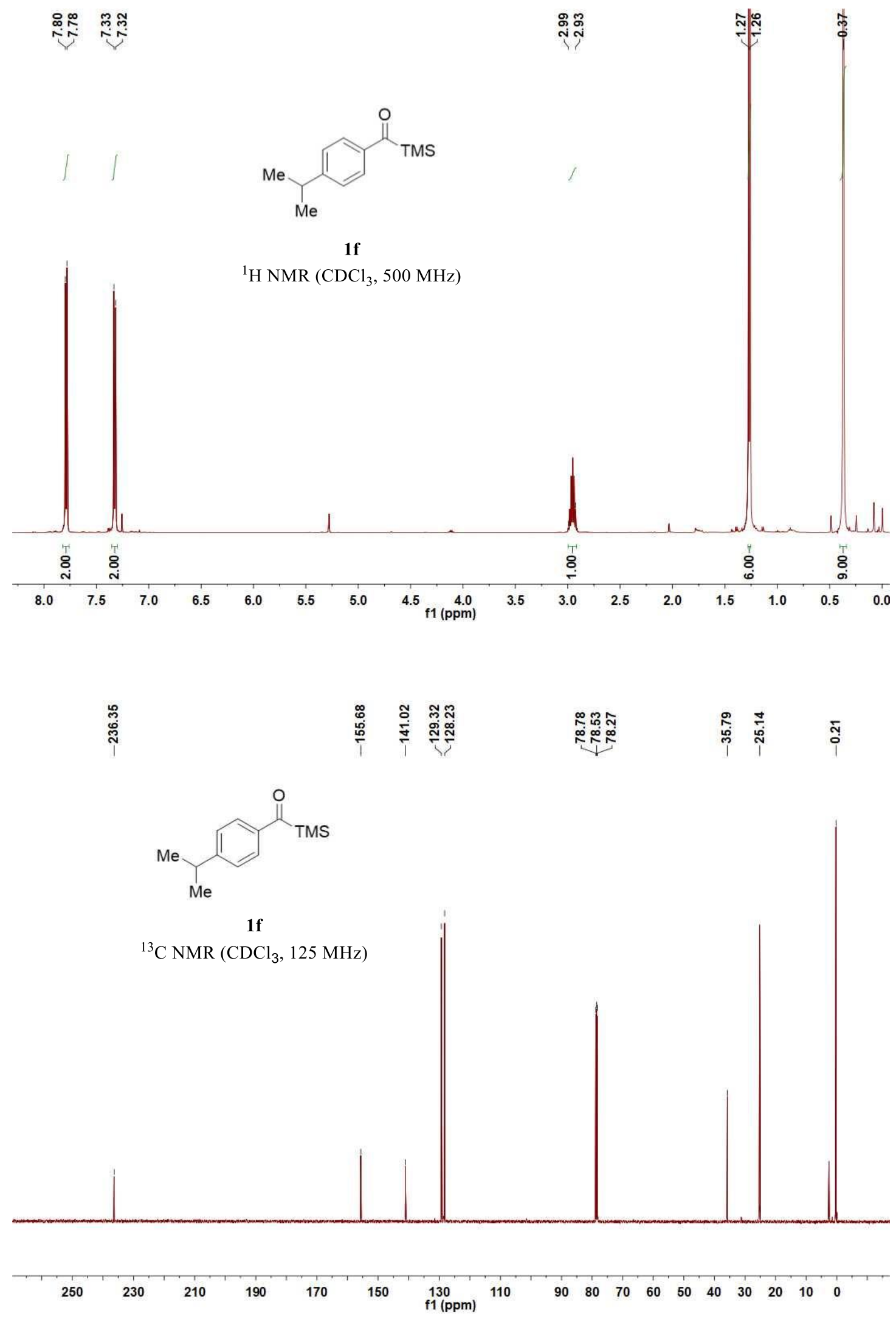

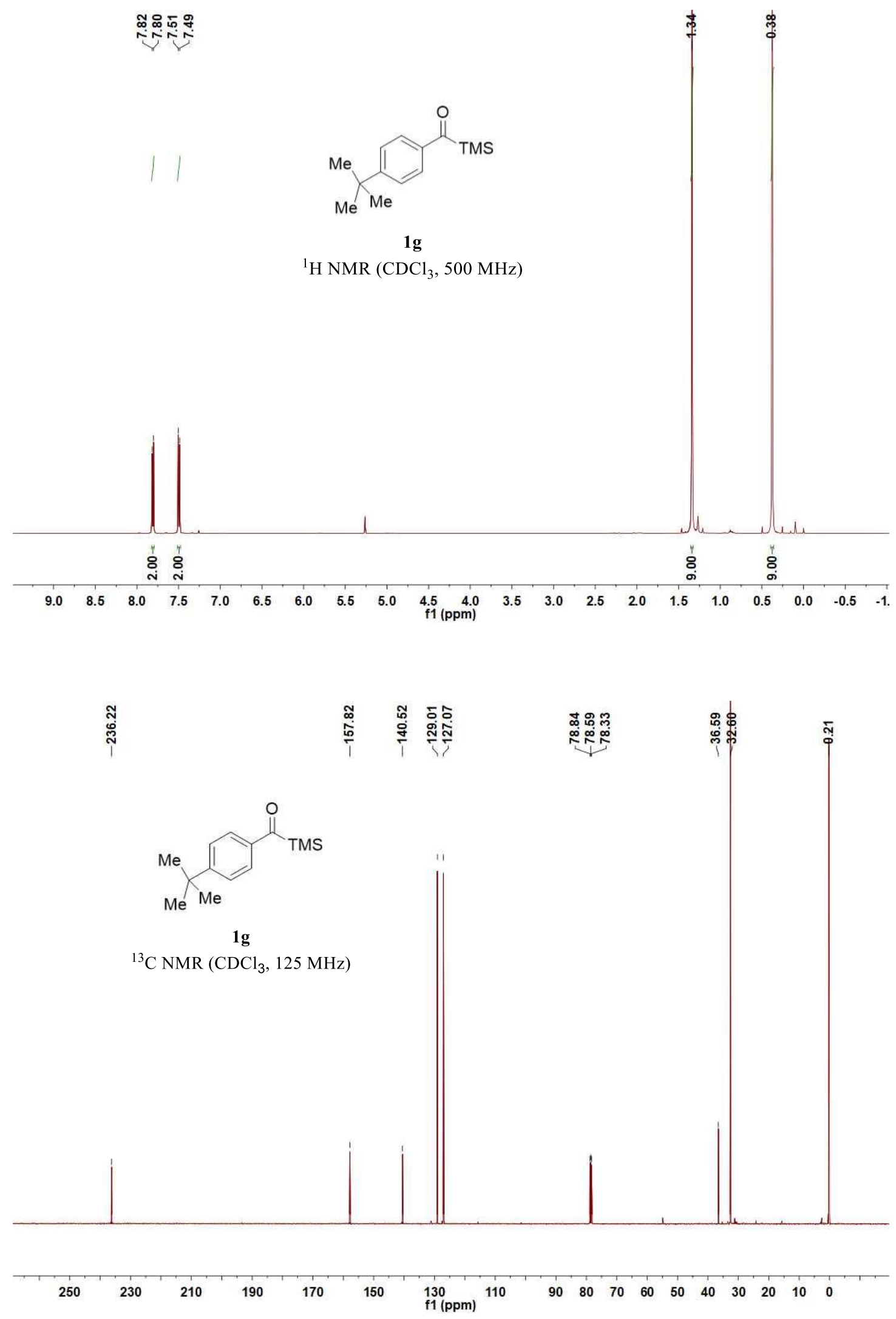


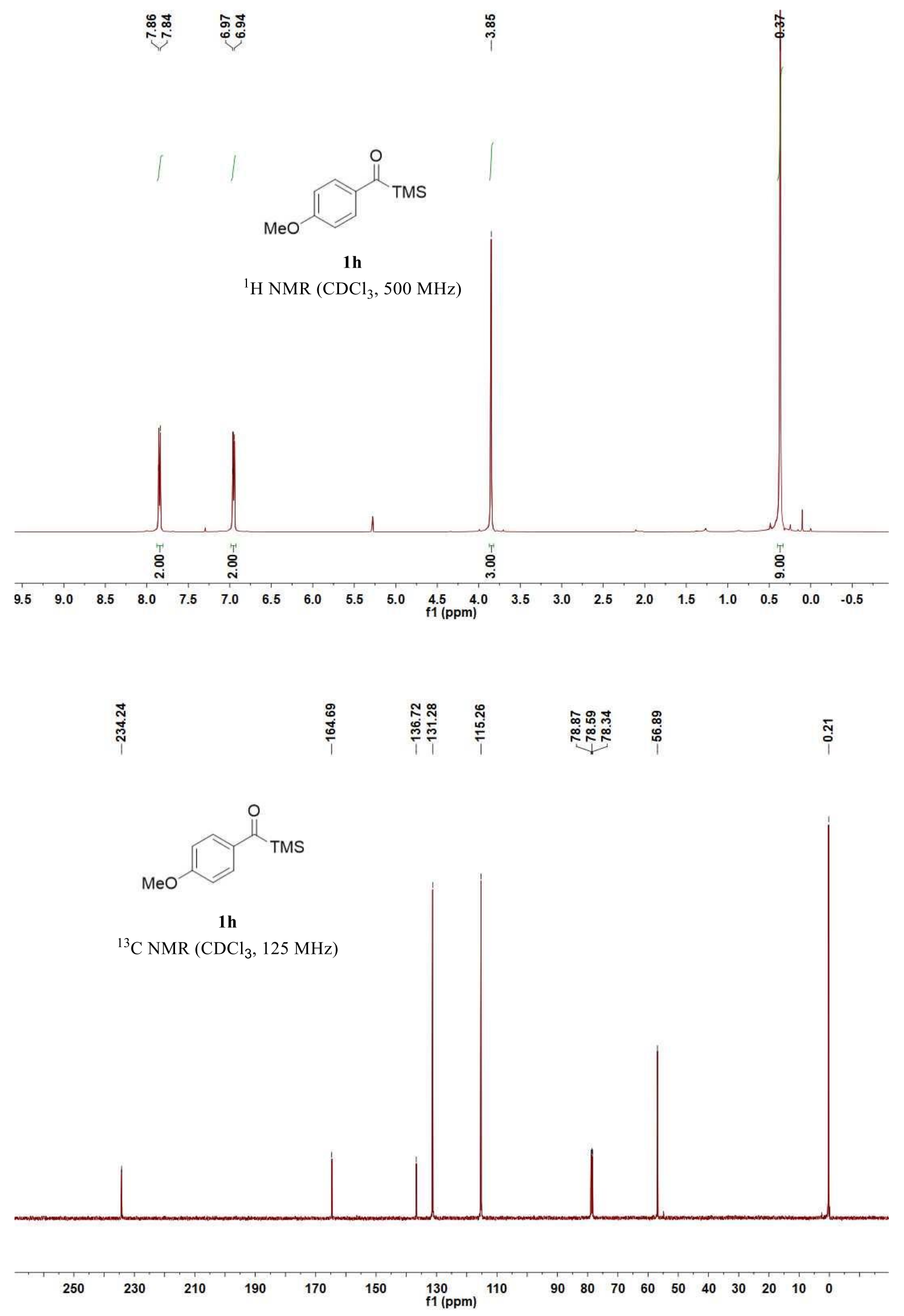




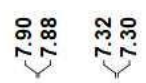

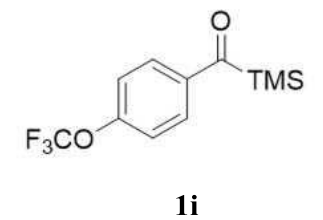

${ }^{1} \mathrm{H} \mathrm{NMR}\left(\mathrm{CDCl}_{3}, 500 \mathrm{MHz}\right)$

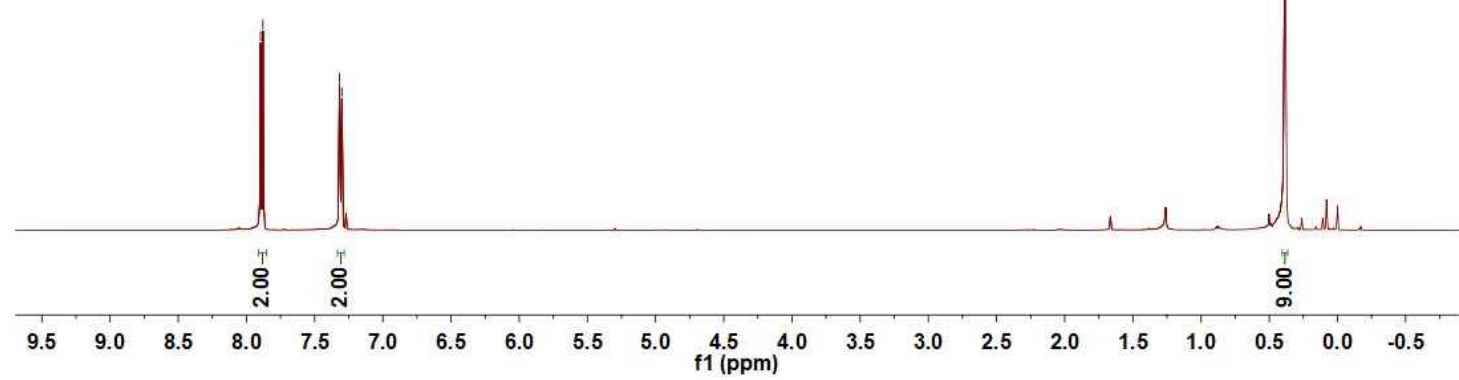

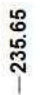

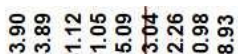

กำ

$\hat{\sin } \times \frac{0}{9}$

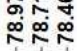

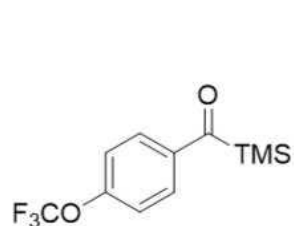

1i

${ }^{13} \mathrm{C}$ NMR $\left(\mathrm{CDCl}_{3}, 125 \mathrm{MHz}\right)$

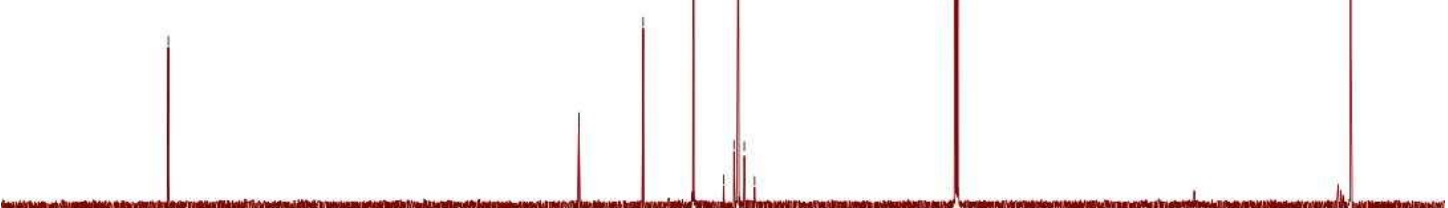



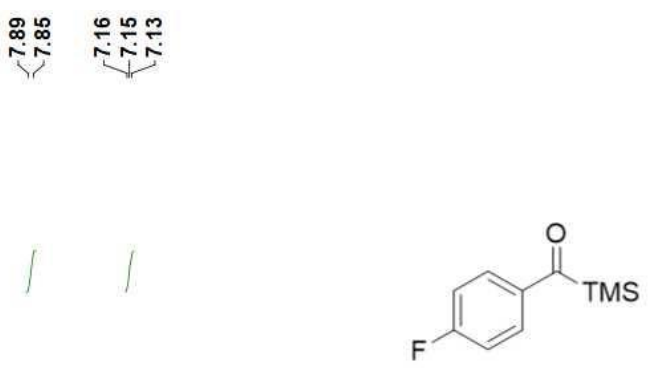

$\mathbf{1 j}$

${ }^{1} \mathrm{H} \mathrm{NMR}\left(\mathrm{CDCl}_{3}, 500 \mathrm{MHz}\right)$

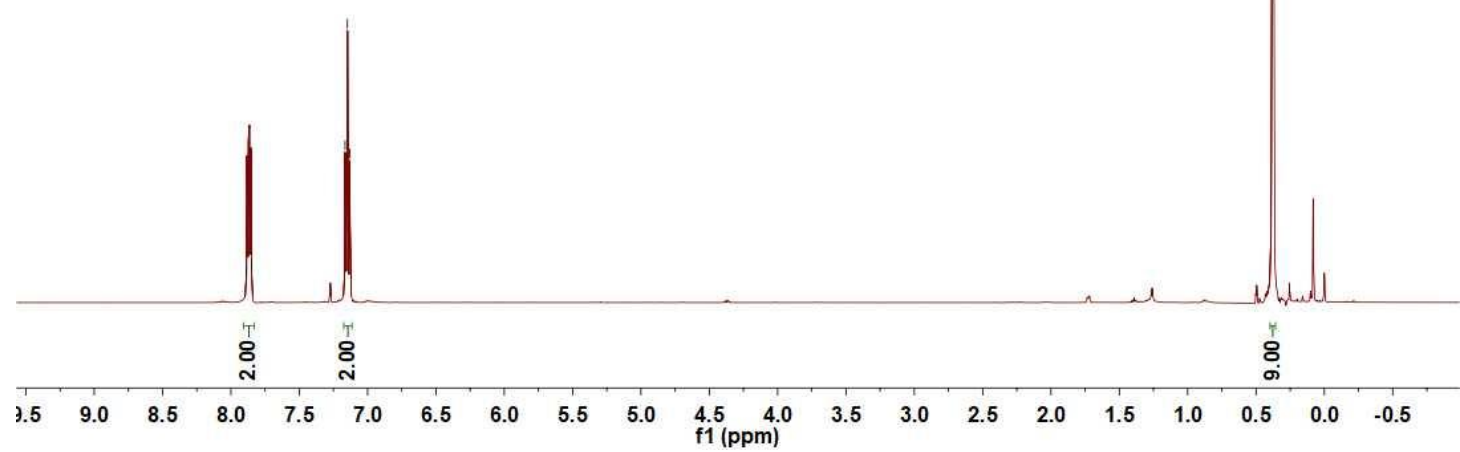

$\underset{\substack{\text { i } \\ \text { ז }}}{i}$

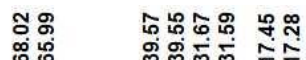

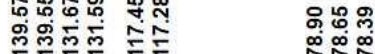

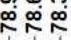

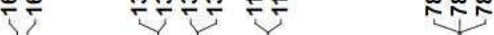

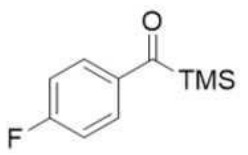

$\mathbf{1 j}$

${ }^{13} \mathrm{C} \mathrm{NMR}\left(\mathrm{CDCl}_{3}, 125 \mathrm{MHz}\right)$

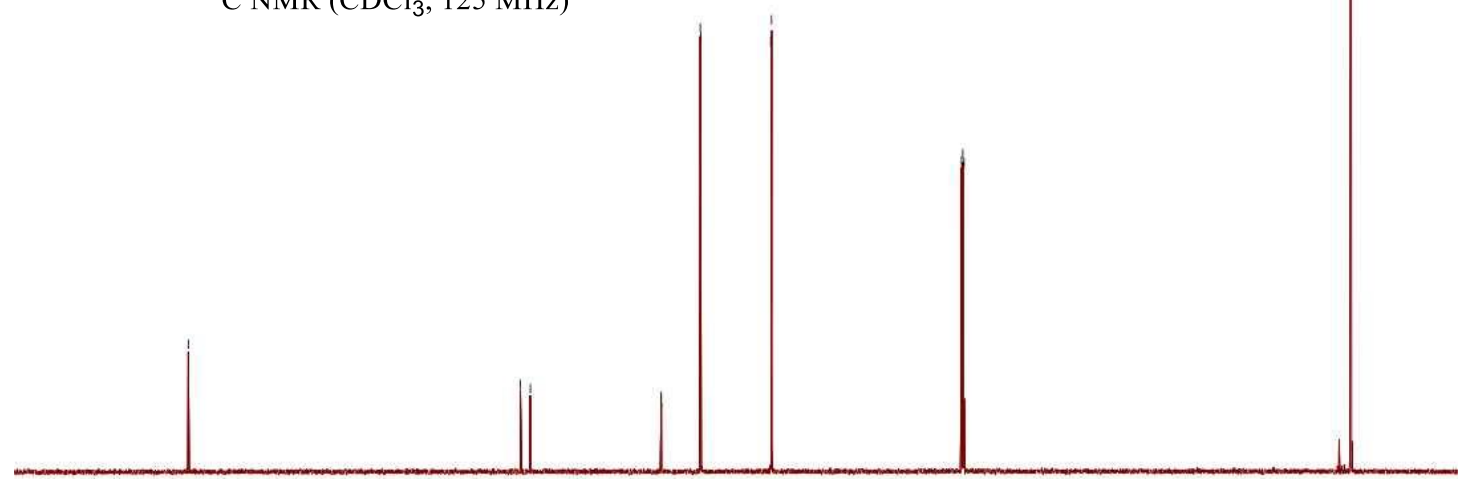

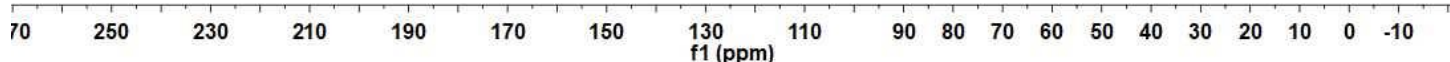



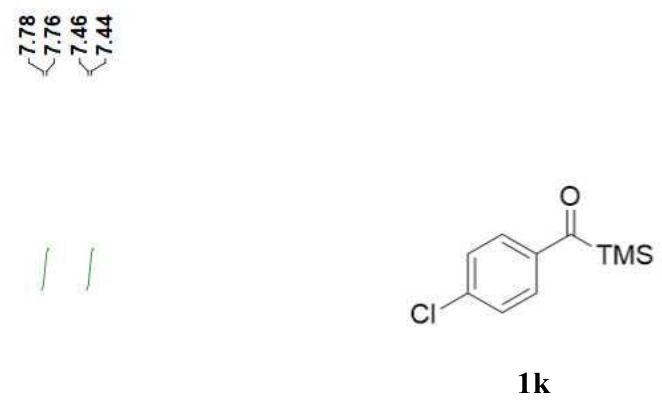

${ }^{1} \mathrm{H} \mathrm{NMR}\left(\mathrm{CDCl}_{3}, 500 \mathrm{MHz}\right)$

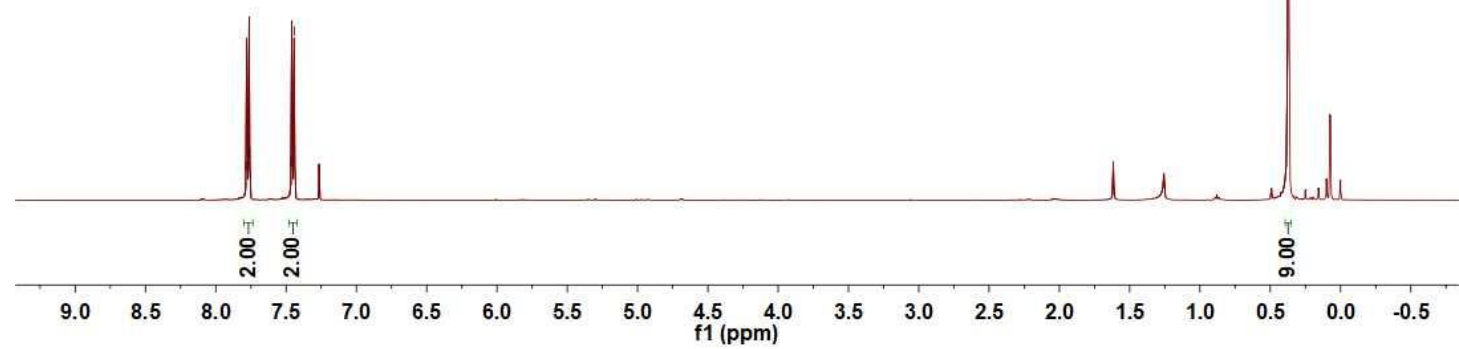

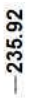

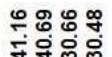

宇熍

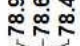

$\underset{\substack{i \\ i}}{\bar{i}}$

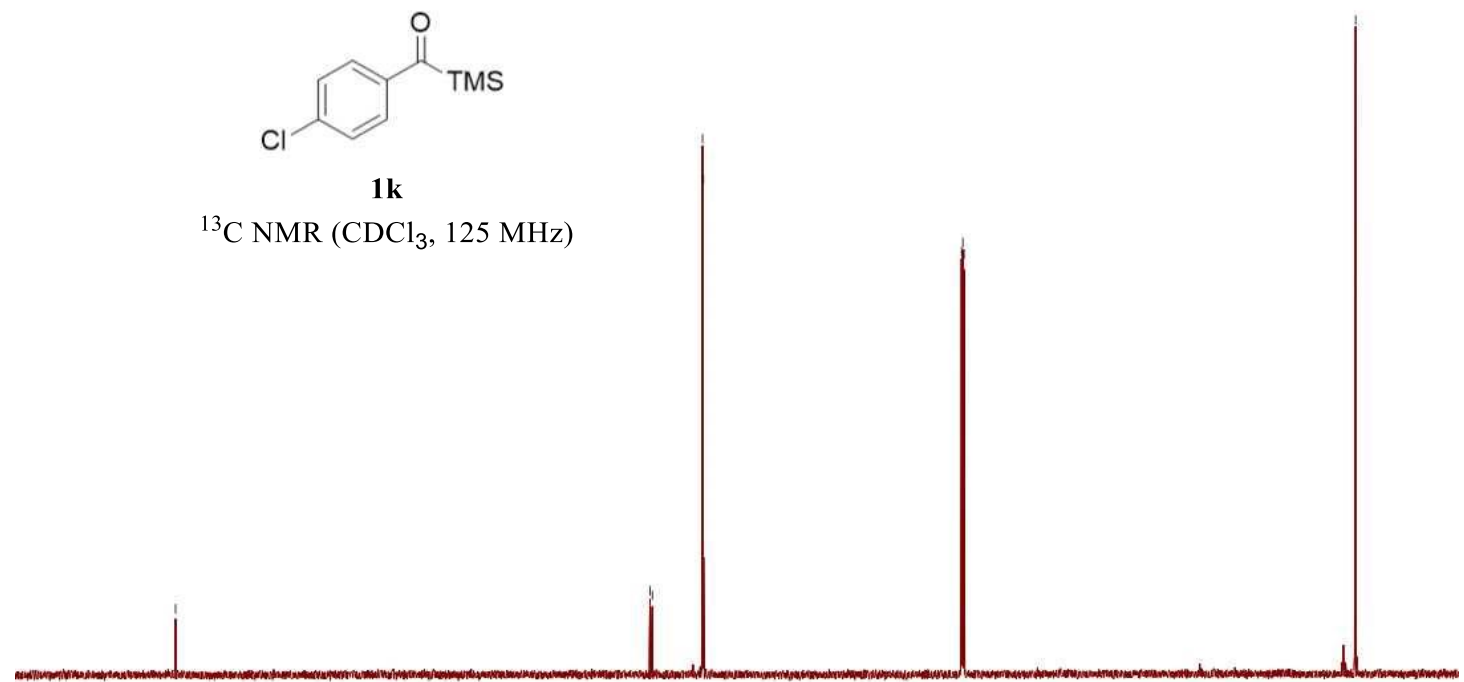

$250 \quad 230 \quad 210 \quad 190 \quad 170 \quad 150 \quad \begin{aligned} & 130 \\ & \mathrm{f} 1(\mathrm{ppm})\end{aligned} \quad \begin{array}{lllllllllllll}90 & 80 & 70 & 60 & 50 & 40 & 30 & 20 & 10 & 0 & -10\end{array}$ 


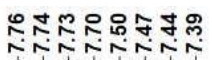

II II

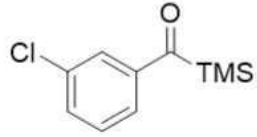

11

${ }^{1} \mathrm{H} \mathrm{NMR}\left(\mathrm{CDCl}_{3}, 500 \mathrm{MHz}\right)$

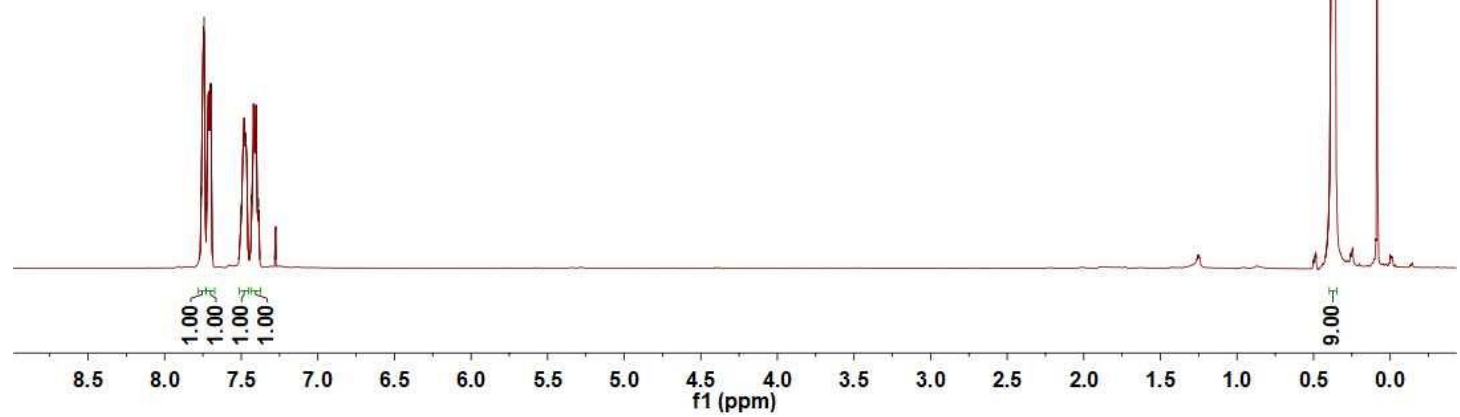

ลิ<smiles>CS(=O)(=O)c1cccc(Cl)c1</smiles>

11

${ }^{13} \mathrm{C}$ NMR $\left(\mathrm{CDCl}_{3}, 125 \mathrm{MHz}\right)$

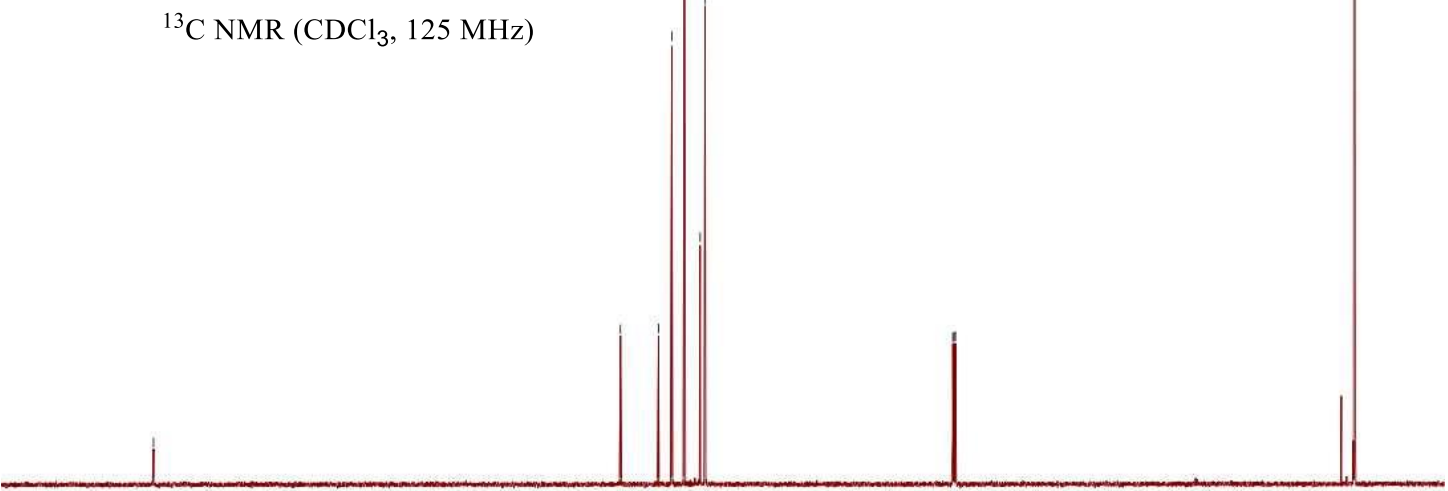

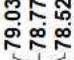

130
$\mathrm{f} 1(\mathrm{ppm})$

$\begin{array}{llllllllll}90 & 80 & 70 & 60 & 50 & 40 & 30 & 20 & 10 & 0\end{array}$ 


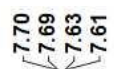

11

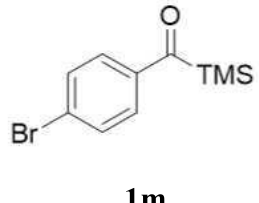

$1 \mathrm{~m}$

${ }^{1} \mathrm{H}$ NMR $\left(\mathrm{CDCl}_{3}, 500 \mathrm{MHz}\right)$

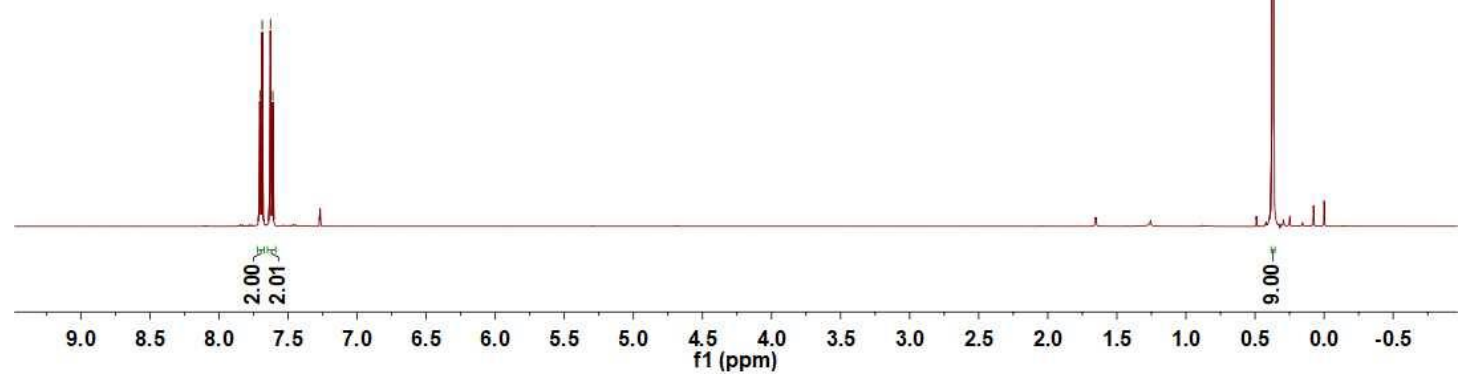

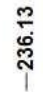

웅유

幽芦

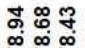

禹央

ז্ড

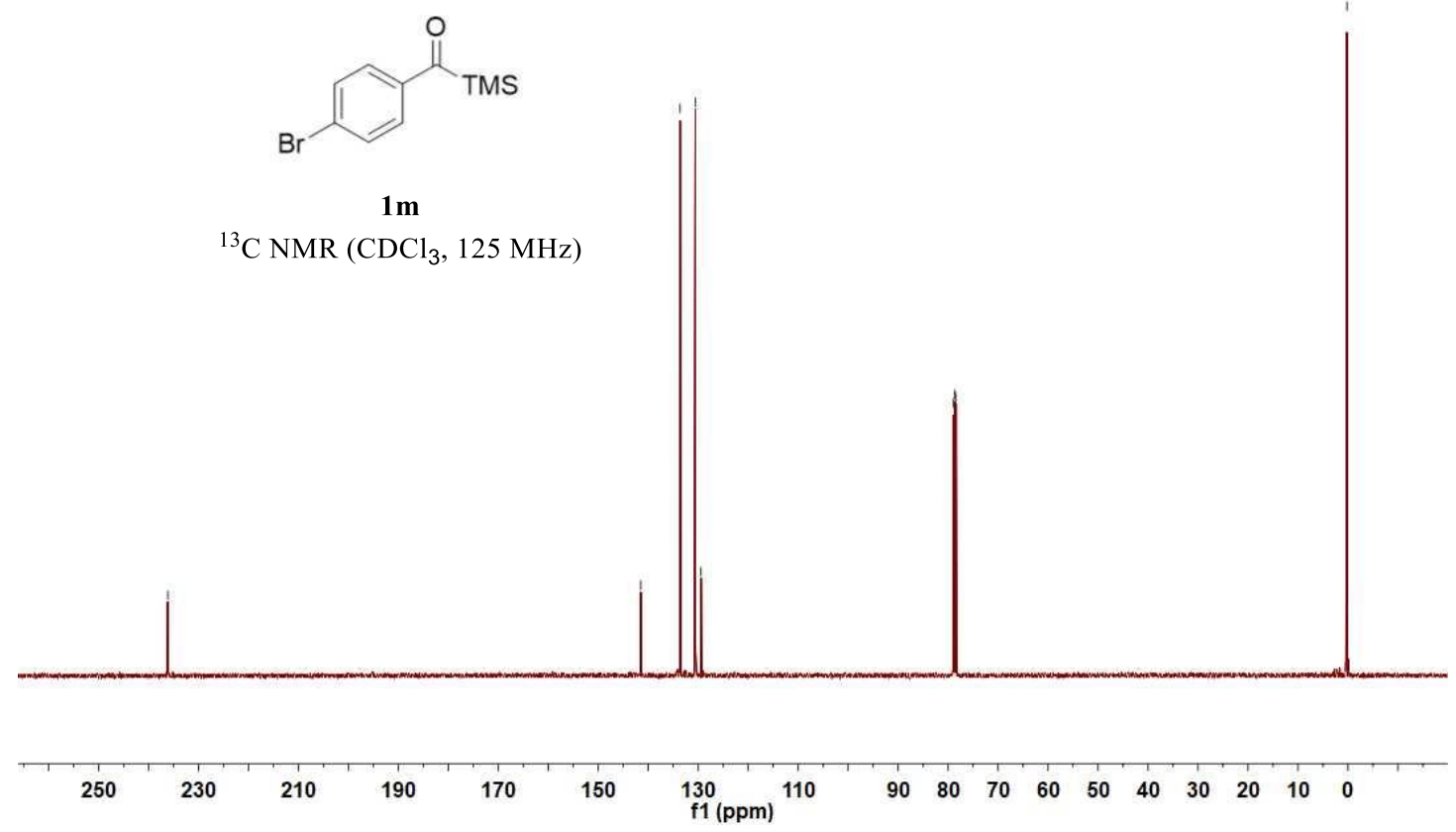




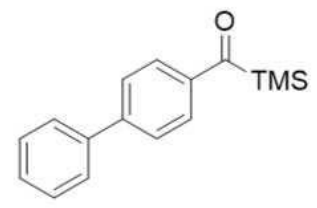

$1 n$

${ }^{1} \mathrm{H}$ NMR $\left(\mathrm{CDCl}_{3}, 500 \mathrm{MHz}\right)$

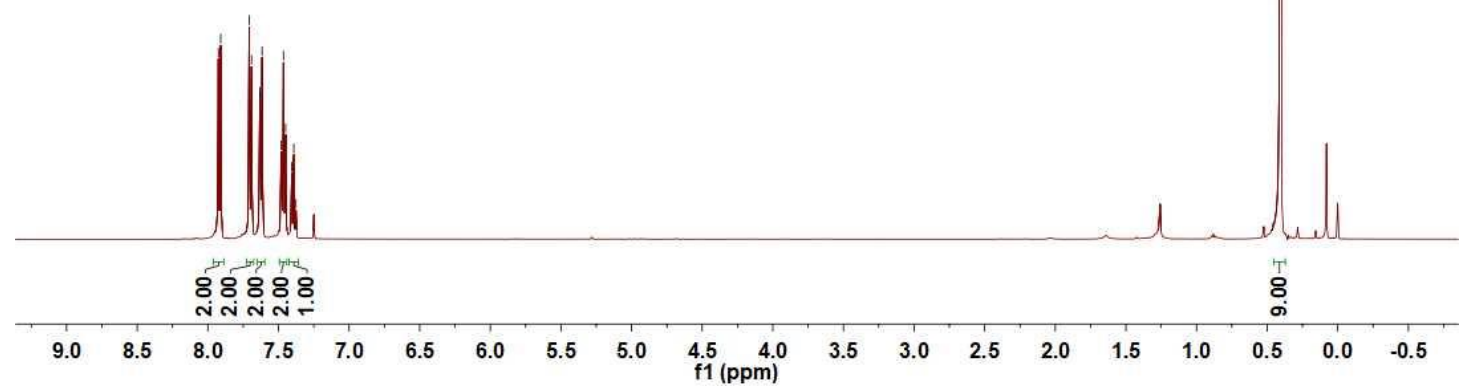

ڤ్ల

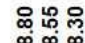

余紧

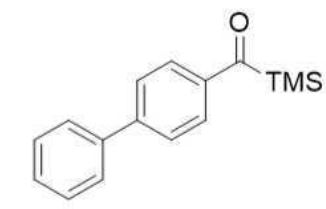

$1 \mathrm{n}$

${ }^{13} \mathrm{C}$ NMR $\left(\mathrm{CDCl}_{3}, 125 \mathrm{MHz}\right)$ 


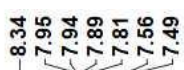

$11 /$

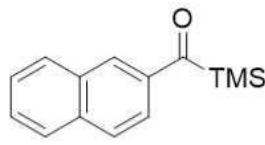

10

${ }^{1} \mathrm{H} \mathrm{NMR}\left(\mathrm{CDCl}_{3}, 500 \mathrm{MHz}\right)$

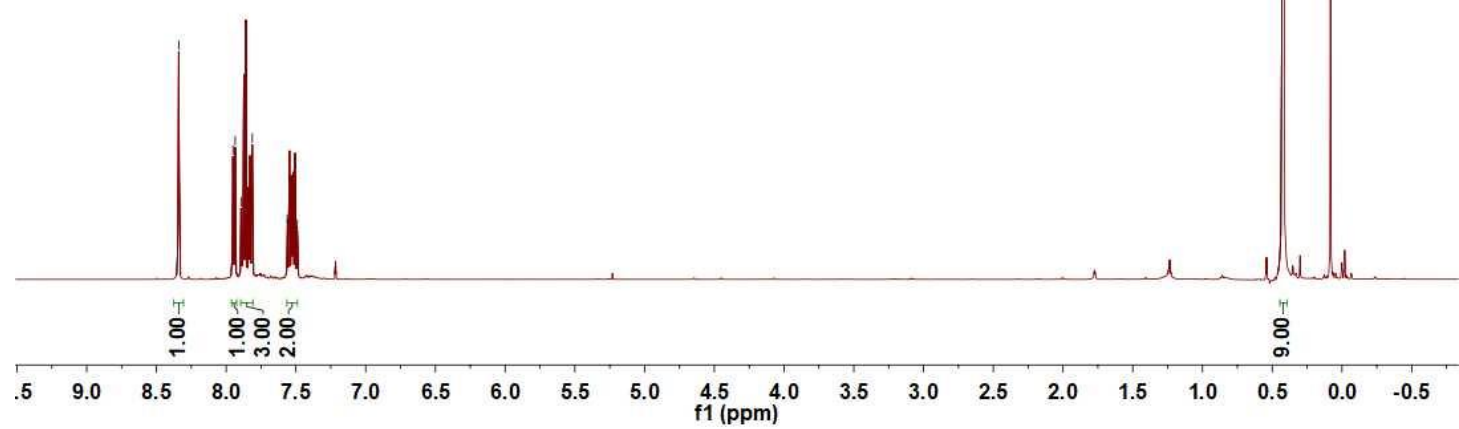

品

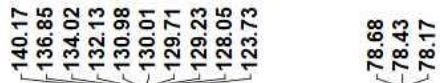

象象

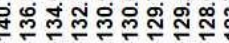

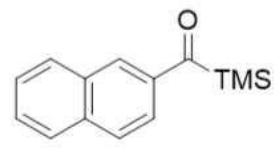

10
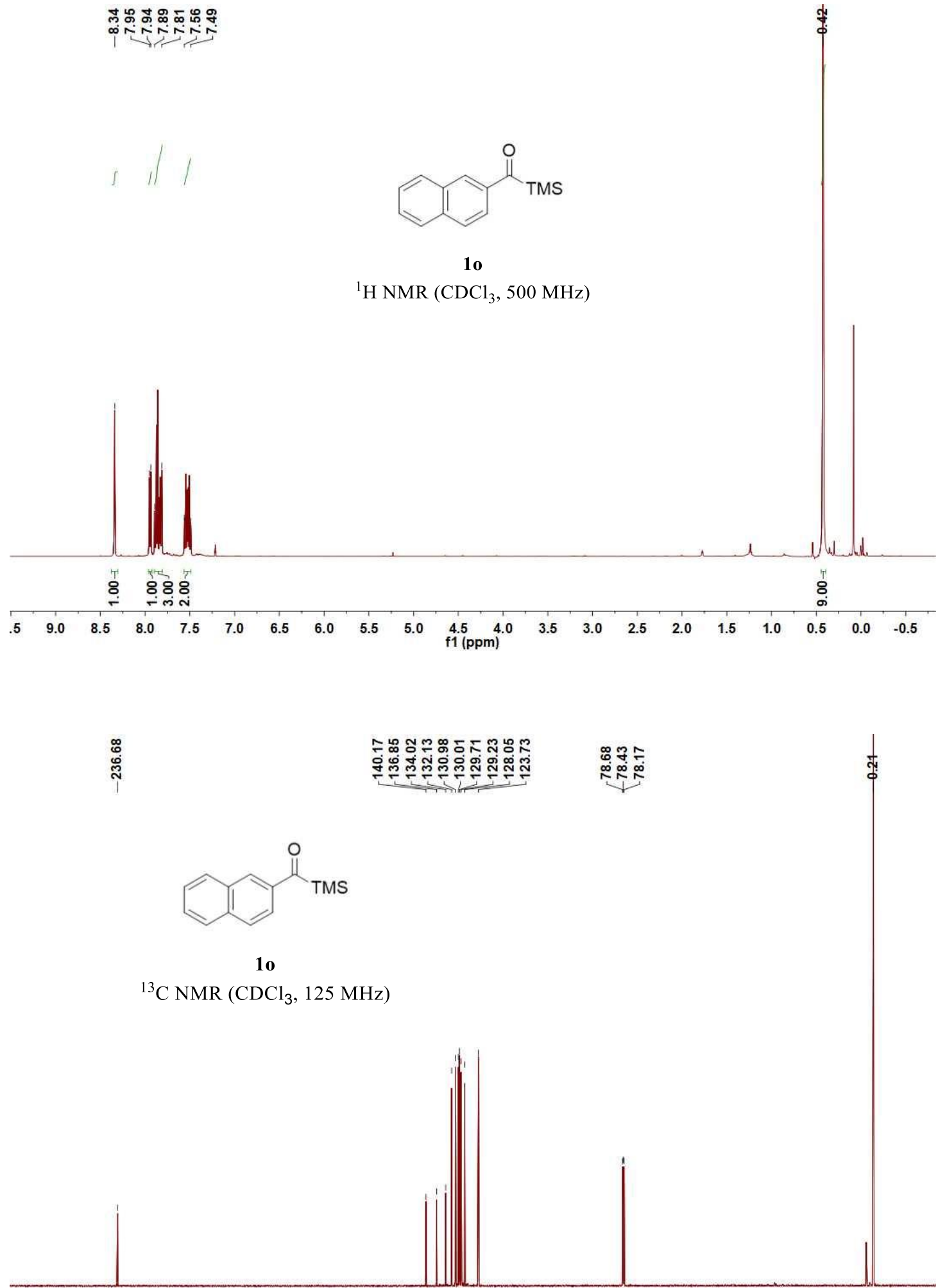

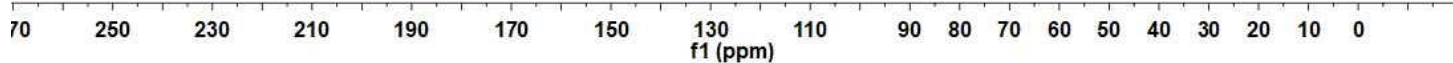


$\stackrel{\text { 学 }}{\stackrel{\tau}{i}}$
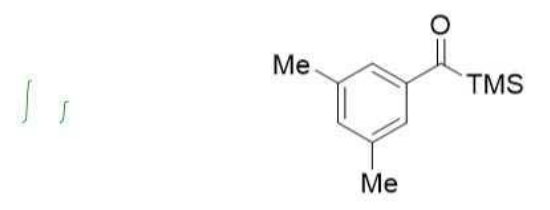

$1 \mathrm{p}$

${ }^{1} \mathrm{H} \mathrm{NMR}\left(\mathrm{CDCl}_{3}, 500 \mathrm{MHz}\right)$

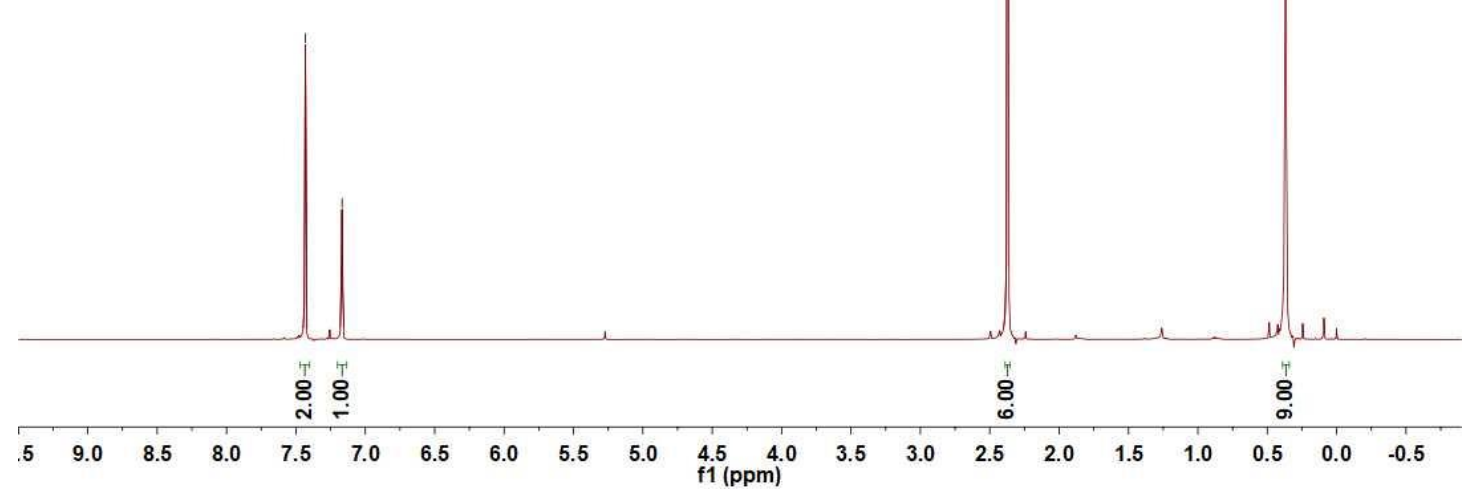

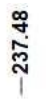

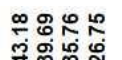

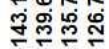

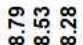

$\infty \cos _{\infty}^{\infty}$

$\stackrel{\substack{1 \\ \text { กิ }}}{i}$<smiles>CC(=O)c1cc(C)cc(C)c1</smiles>

$1 \mathbf{p}$

${ }^{13} \mathrm{C} \mathrm{NMR}\left(\mathrm{CDCl}_{3}, 125 \mathrm{MHz}\right)$

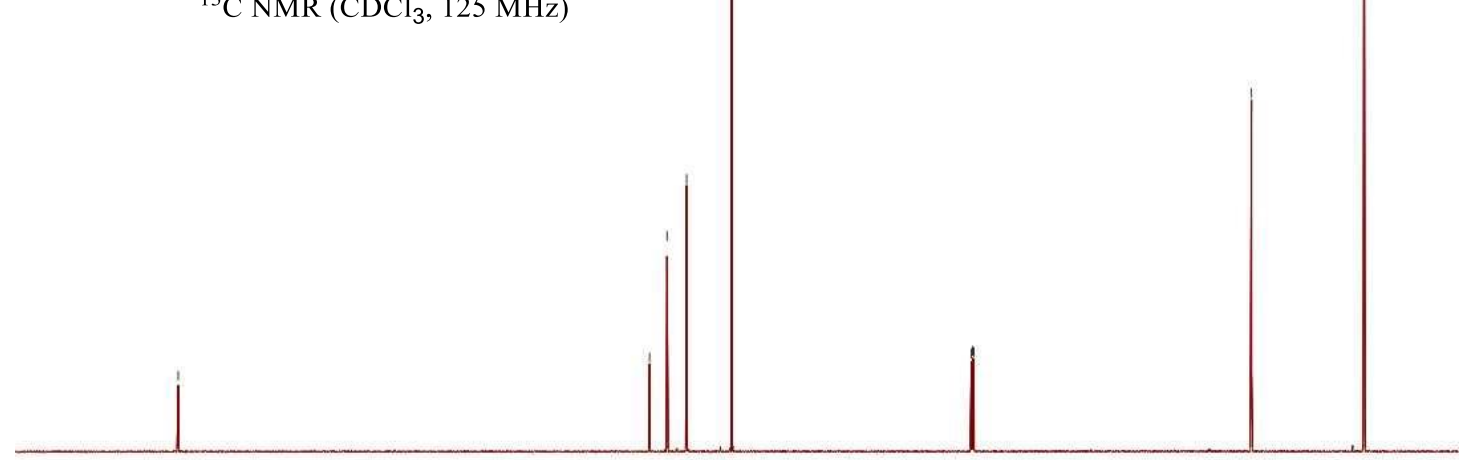

$10 \quad 250 \quad 230$

$190 \quad 170 \quad 150$

130
$\mathrm{f} 1(\mathrm{ppm})$

$\begin{array}{llllllllll}90 & 80 & 70 & 60 & 50 & 40 & 30 & 20 & 10 & 0\end{array}$ 


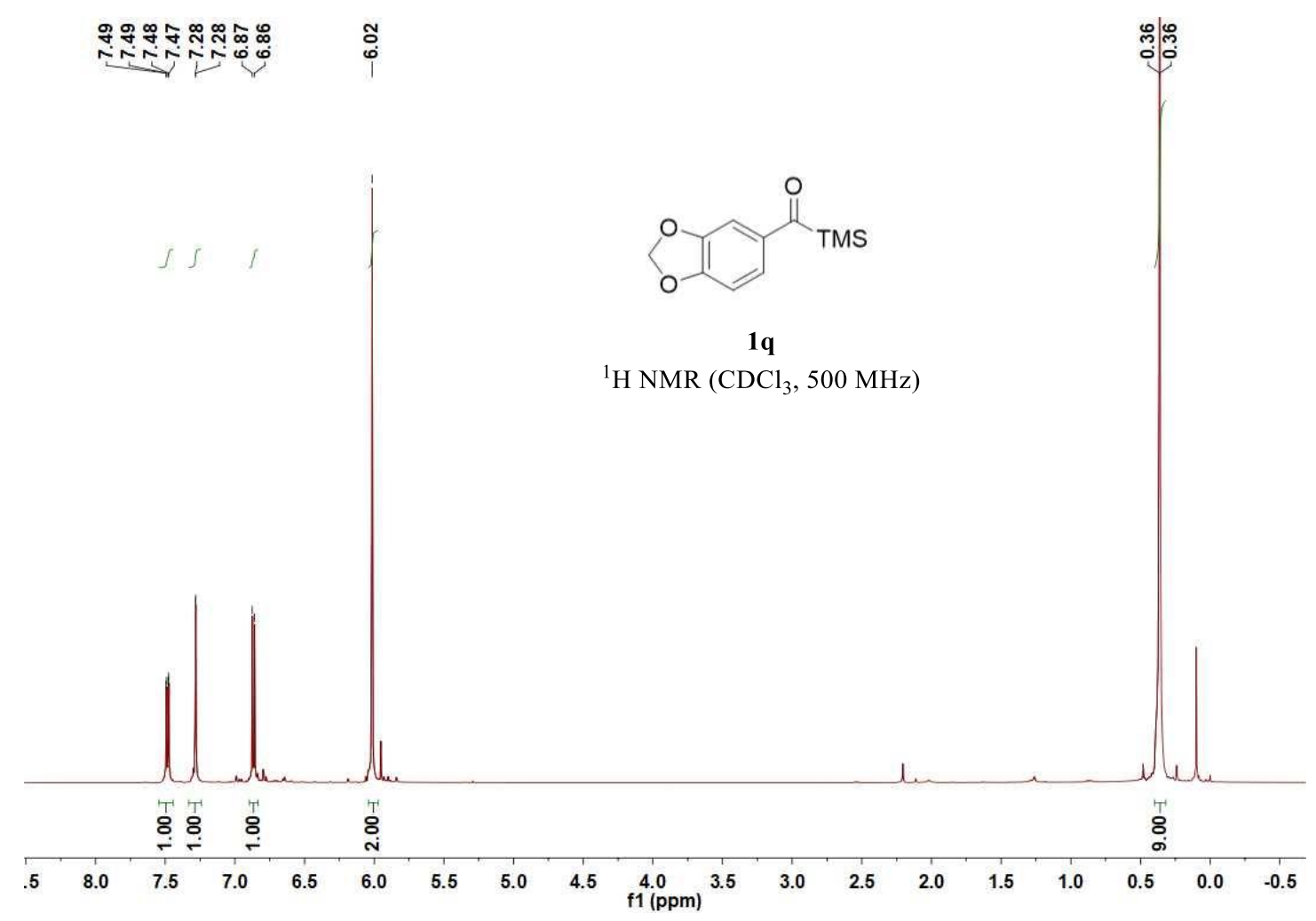

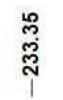
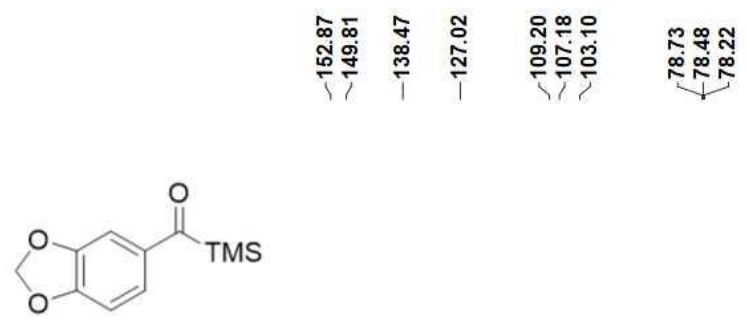

$1 q$

${ }^{13} \mathrm{C} \mathrm{NMR}\left(\mathrm{CDCl}_{3}, 125 \mathrm{MHz}\right)$

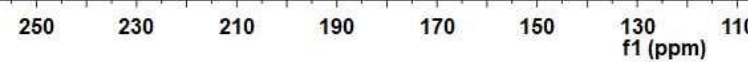

130
$\mathrm{f} 1(\mathrm{ppm})$

$\begin{array}{llllllllll}90 & 80 & 70 & 60 & 50 & 40 & 30 & 20 & 10 & 0\end{array}$ 


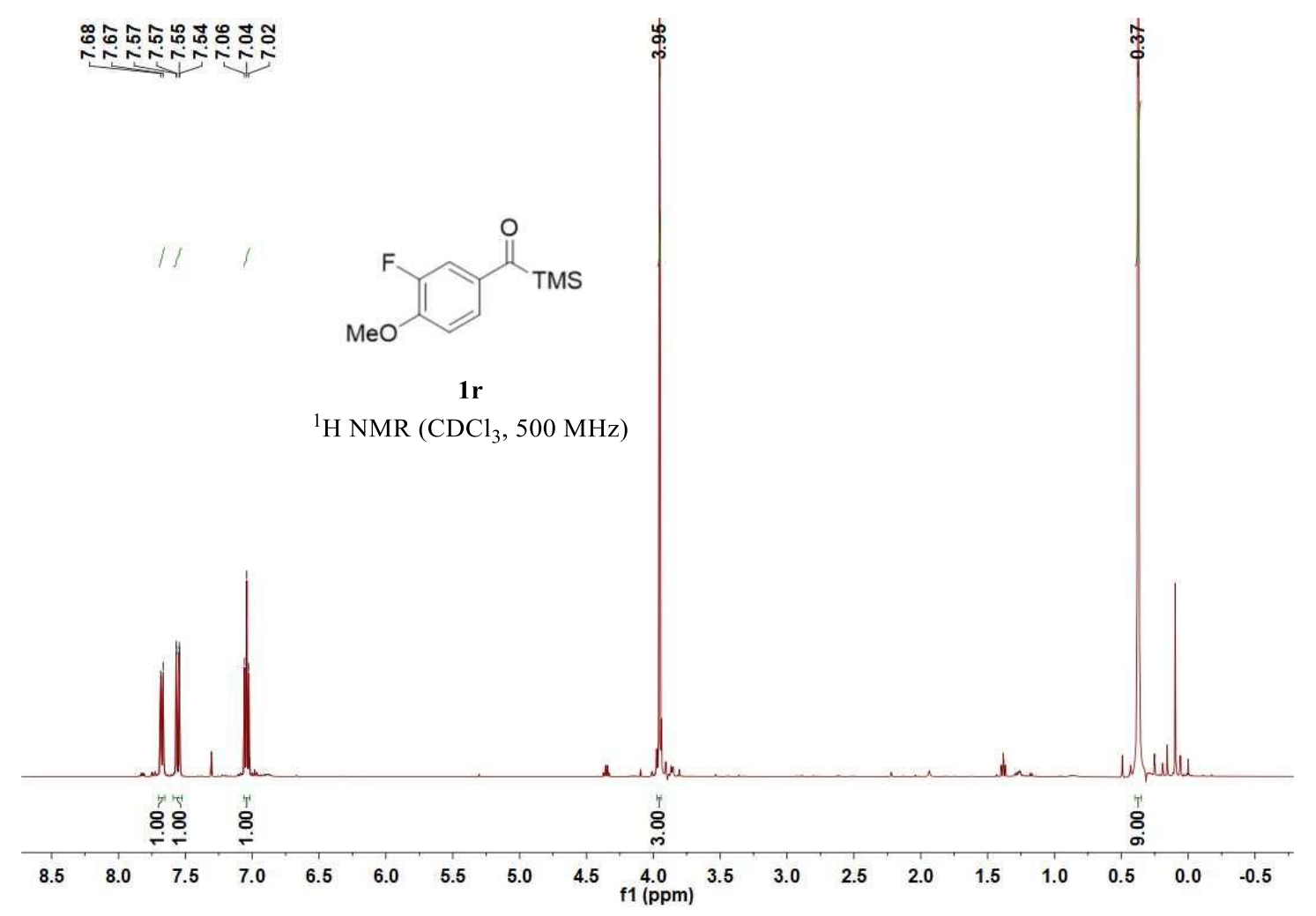

กิ

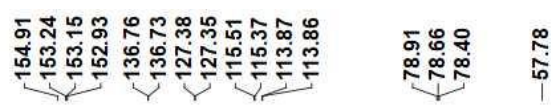

$\stackrel{\infty}{\stackrel{\infty}{\varphi}}$
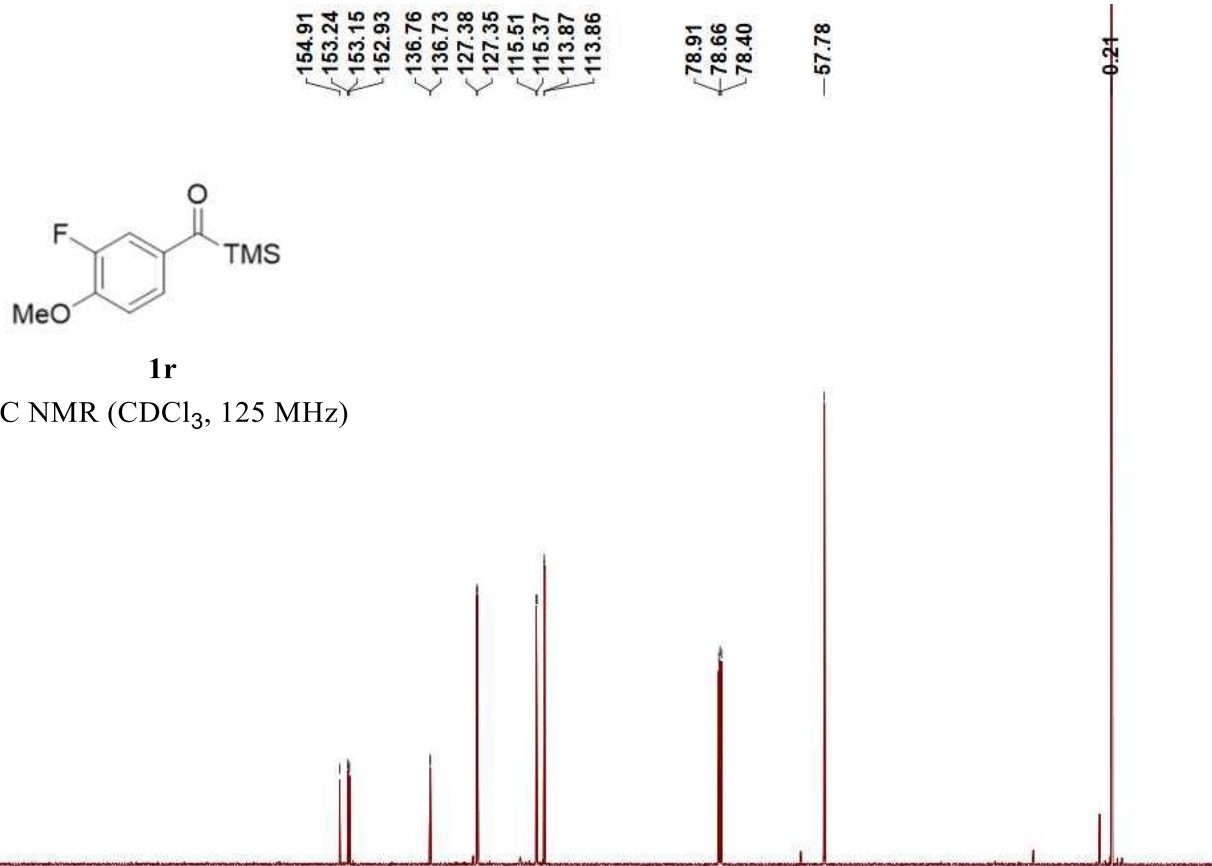

$1 \mathbf{r}$

${ }^{13} \mathrm{C} \mathrm{NMR}\left(\mathrm{CDCl}_{3}, 125 \mathrm{MHz}\right)$

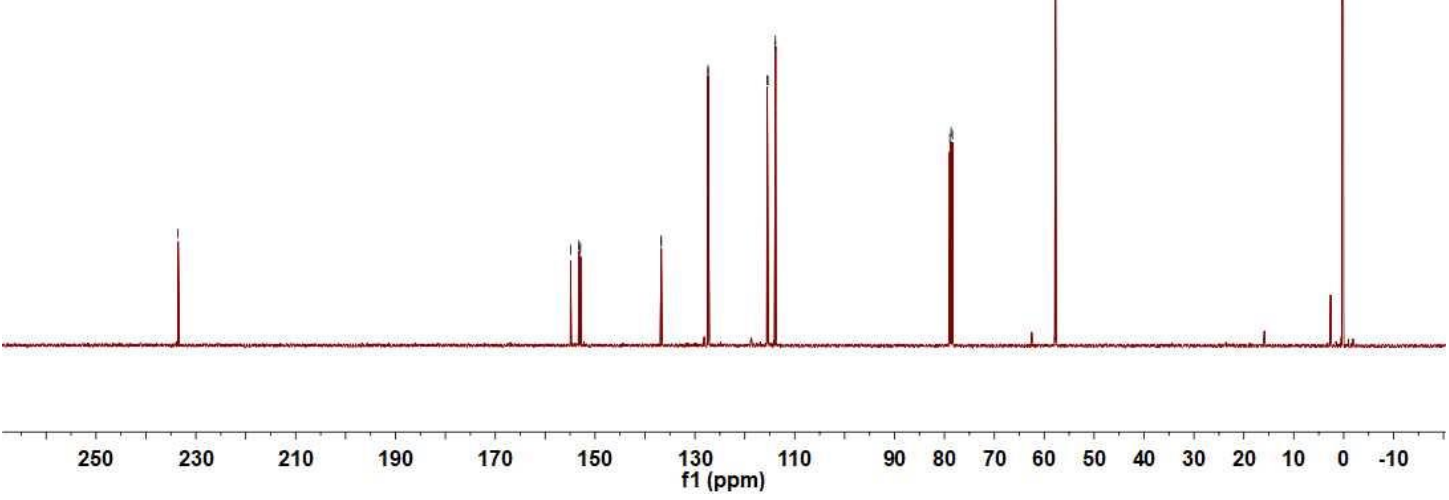



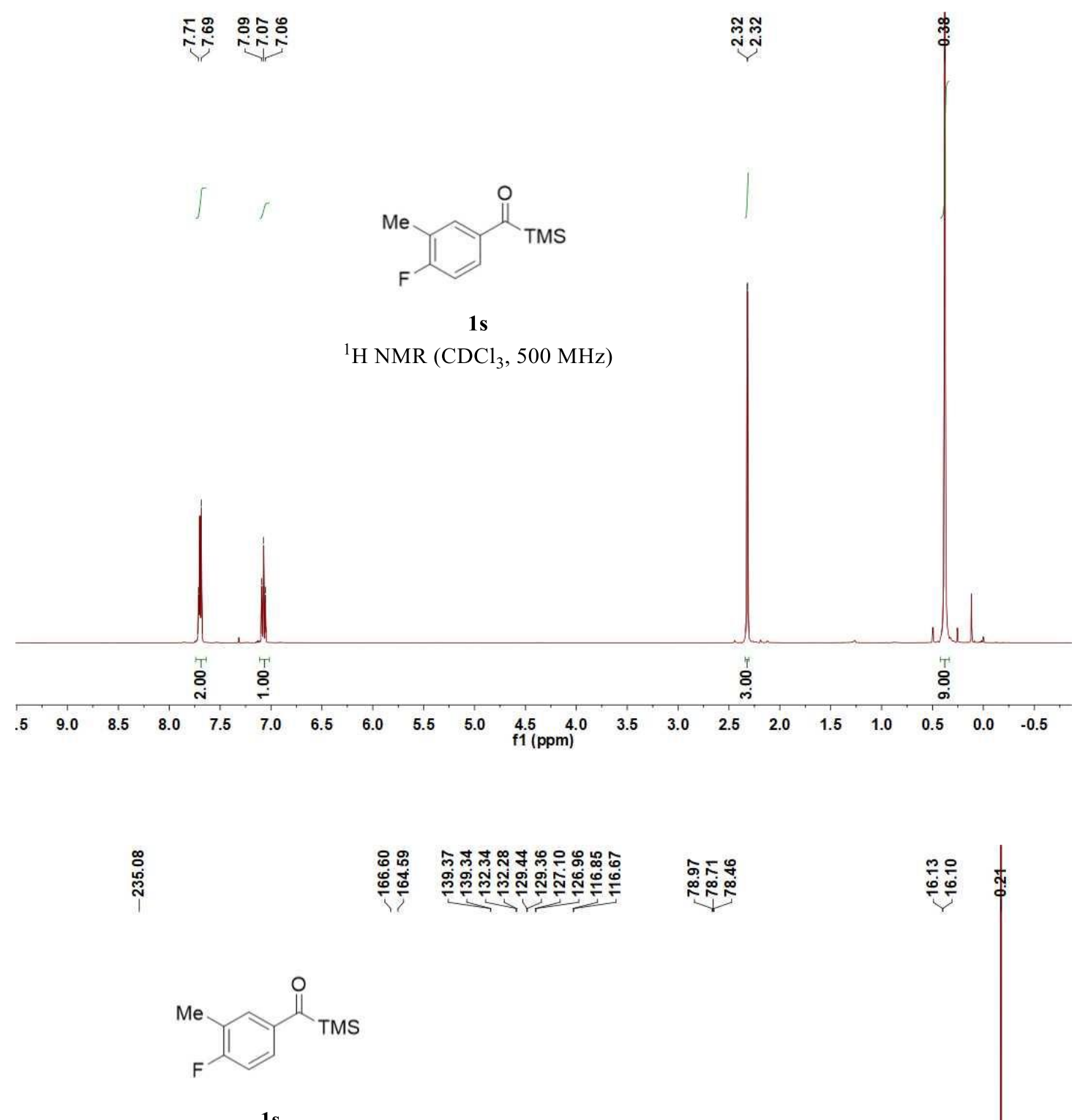

${ }^{13} \mathrm{C} \mathrm{NMR}\left(\mathrm{CDCl}_{3}, 125 \mathrm{MHz}\right)$

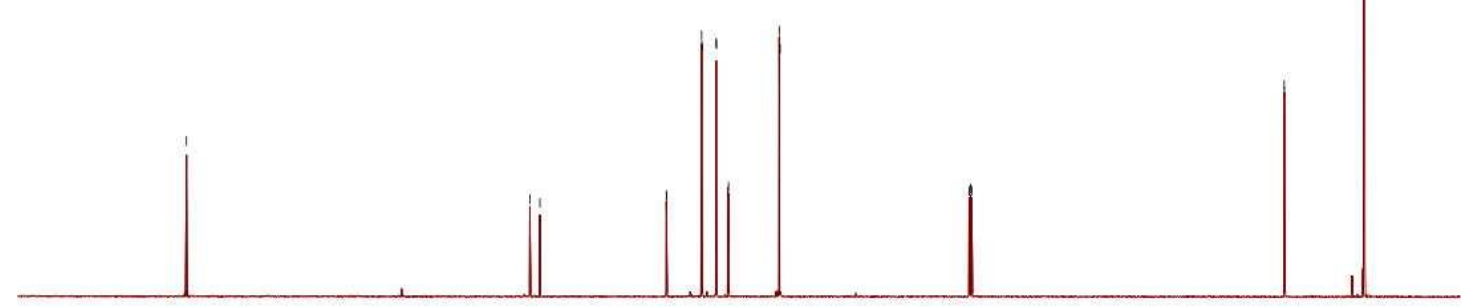

$\begin{array}{lllllllllllllllllllllllll}250 & 230 & 210 & 190 & 170 & 150 & 130 & 110 & 90 & 80 & 70 & 60 & 50 & 40 & 30 & 20 & 10 & 0\end{array}$ 


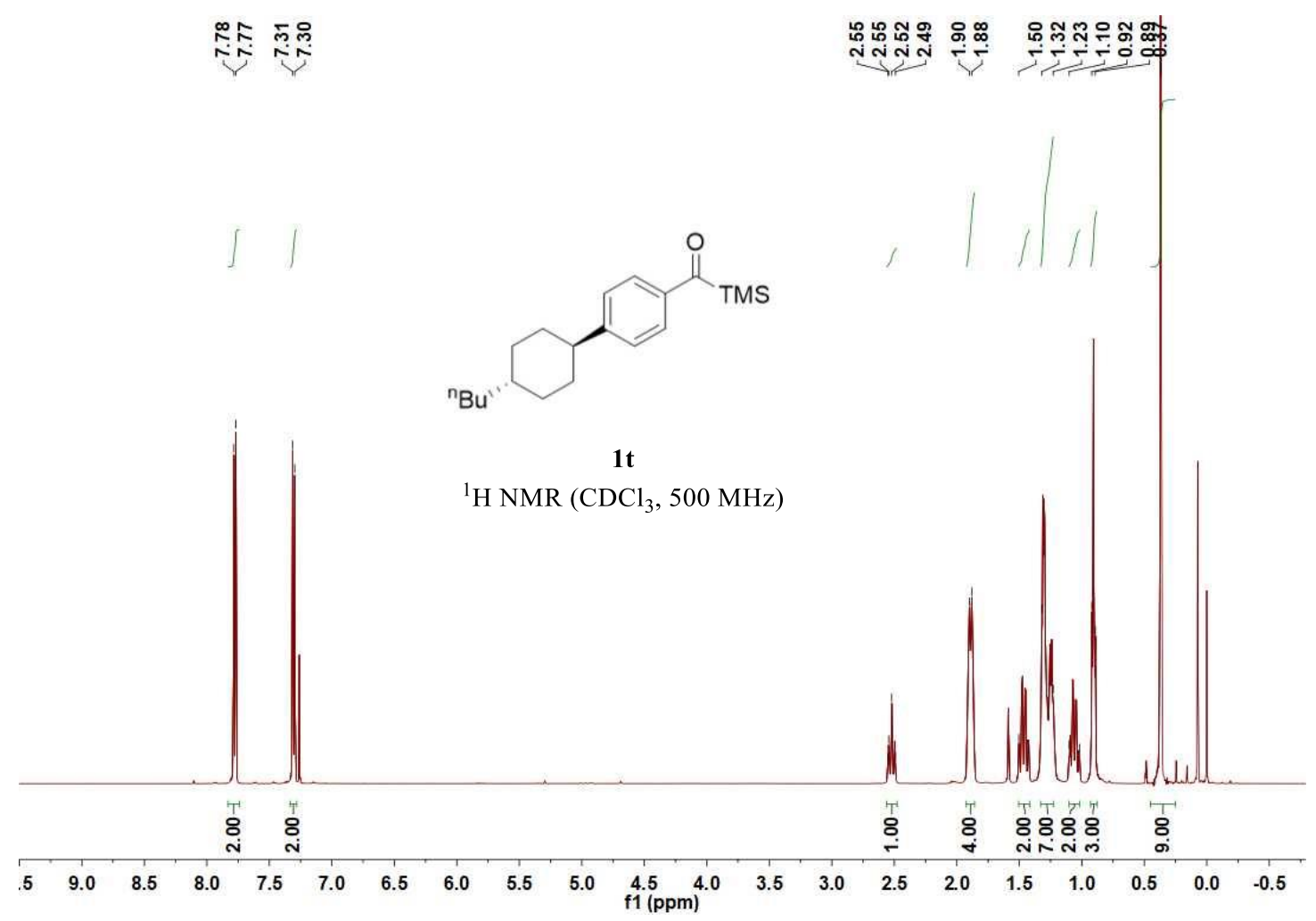

常

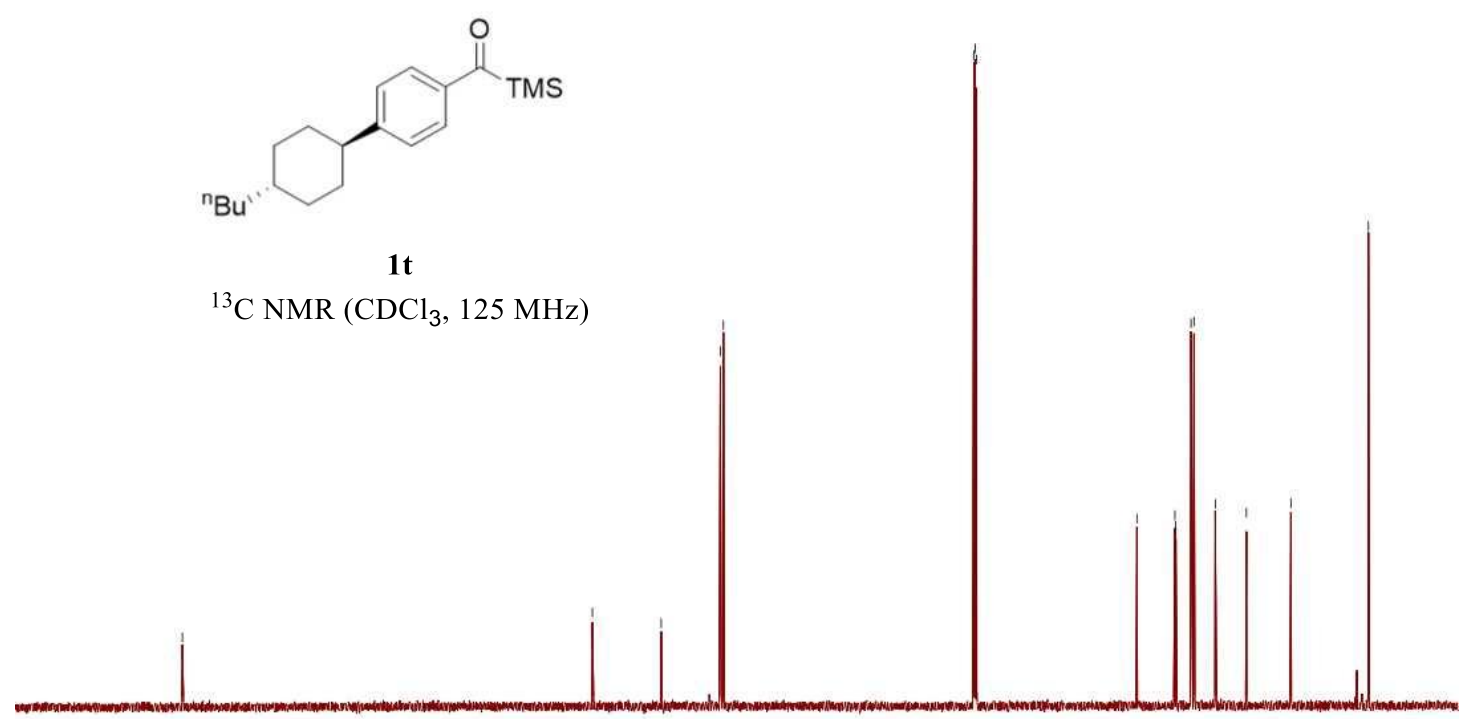

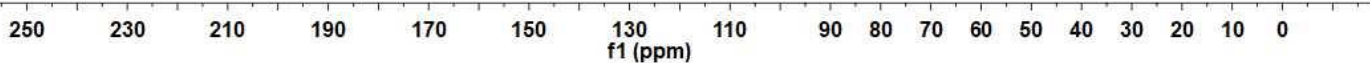



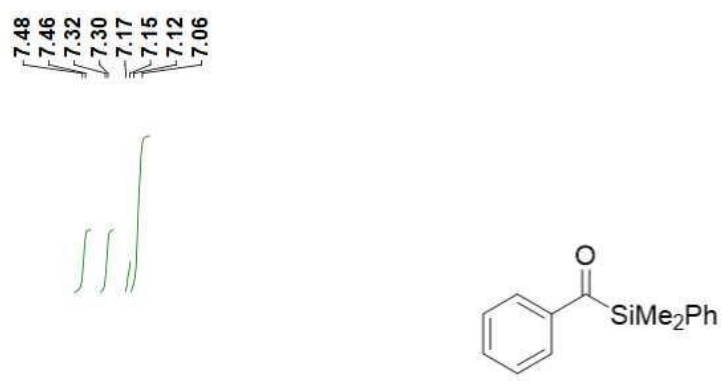

1u

${ }^{1} \mathrm{H} \mathrm{NMR}\left(\mathrm{CDCl}_{3}, 500 \mathrm{MHz}\right)$

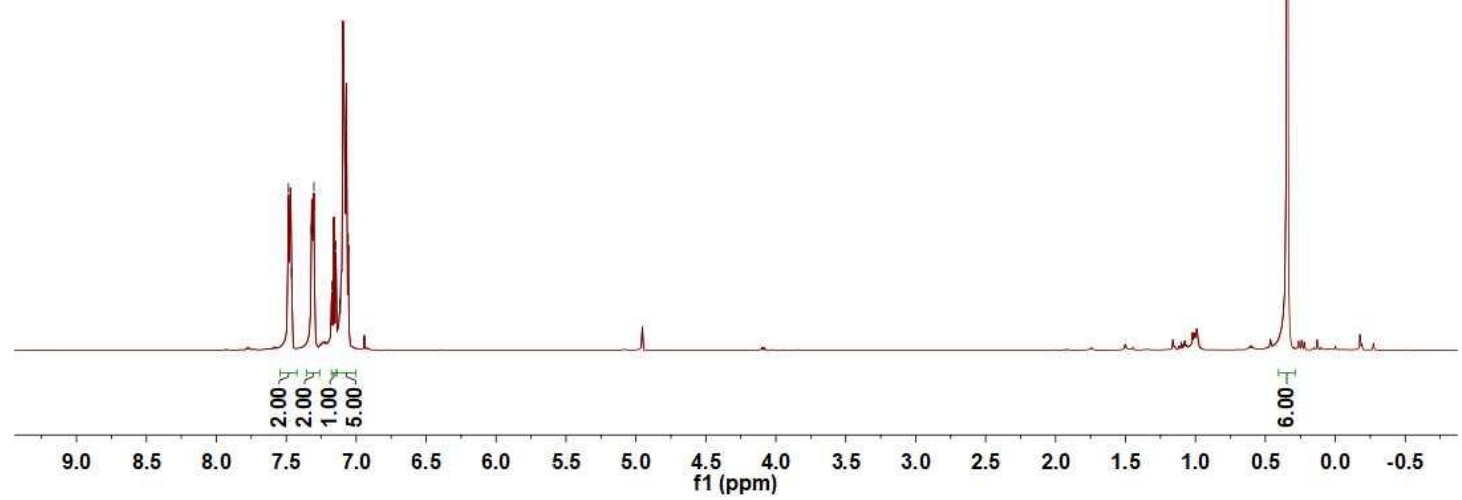

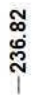

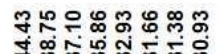

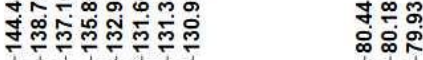

สั

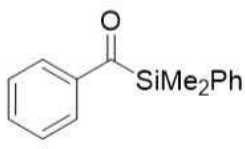

1u

${ }^{13} \mathrm{C} \mathrm{NMR}\left(\mathrm{CDCl}_{3}, 125 \mathrm{MHz}\right)$

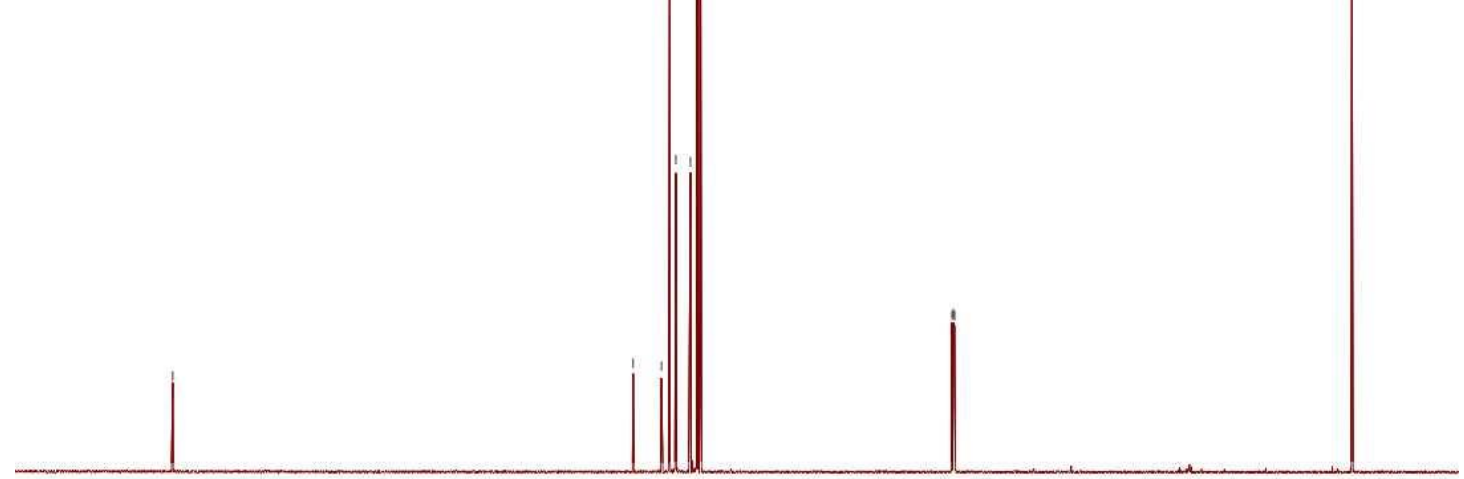

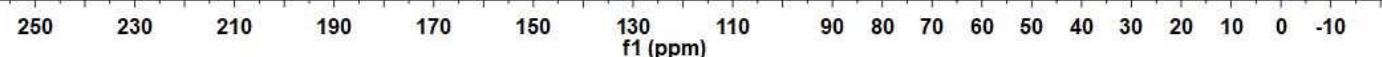




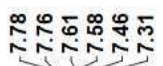

i்
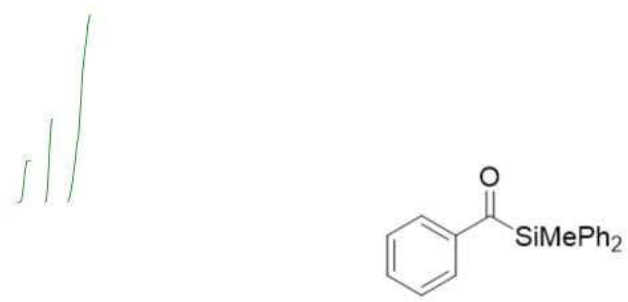

$1 \mathbf{v}$

${ }^{1} \mathrm{H} \mathrm{NMR}\left(\mathrm{CDCl}_{3}, 500 \mathrm{MHz}\right)$

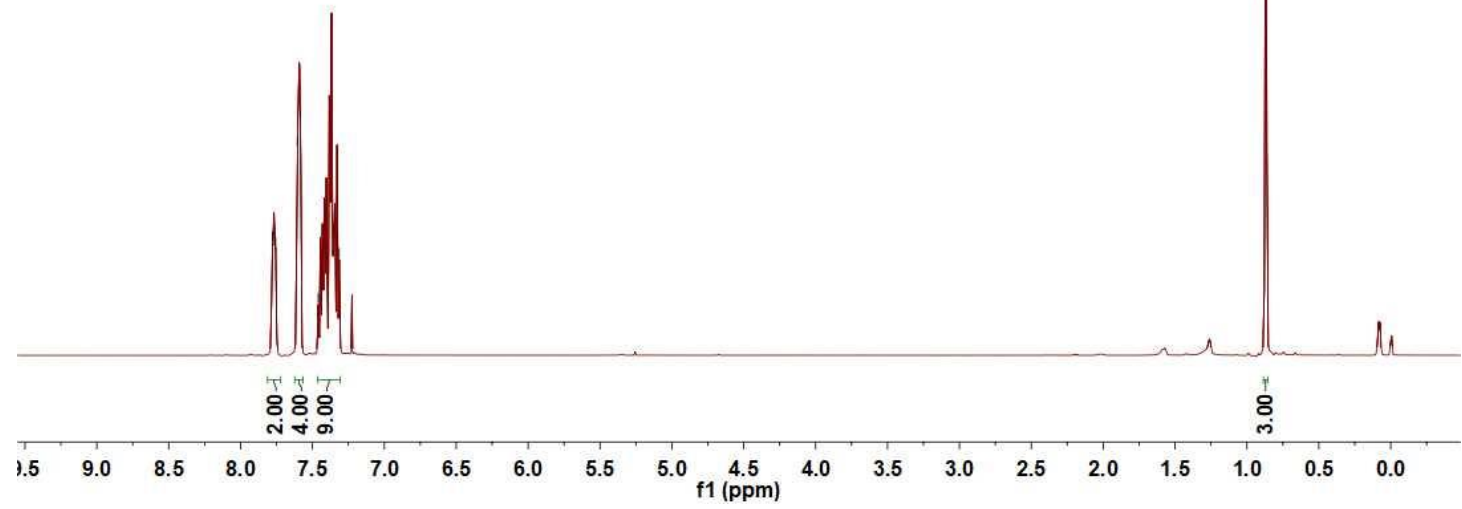

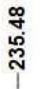

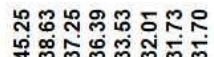

年

๓ं ळ.

ลัก

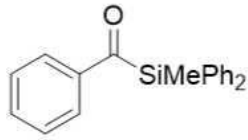

1v

${ }^{13} \mathrm{C} \mathrm{NMR}\left(\mathrm{CDCl}_{3}, 125 \mathrm{MHz}\right)$
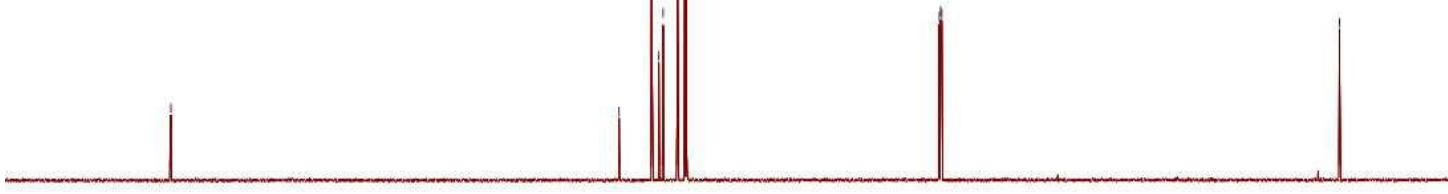

$250 \quad 230 \quad 210 \quad 190$

$\begin{array}{lll}170 & 150 & 130 \\ \mathrm{f} 1(\mathrm{ppm}) & 110\end{array}$

$\begin{array}{lllllllllll}90 & 80 & 70 & 60 & 50 & 40 & 30 & 20 & 10 & 0 & -10\end{array}$ 


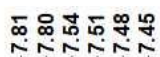

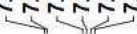

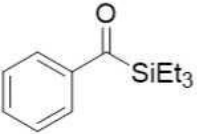

1w

${ }^{1} \mathrm{H} \mathrm{NMR}\left(\mathrm{CDCl}_{3}, 500 \mathrm{MHz}\right)$

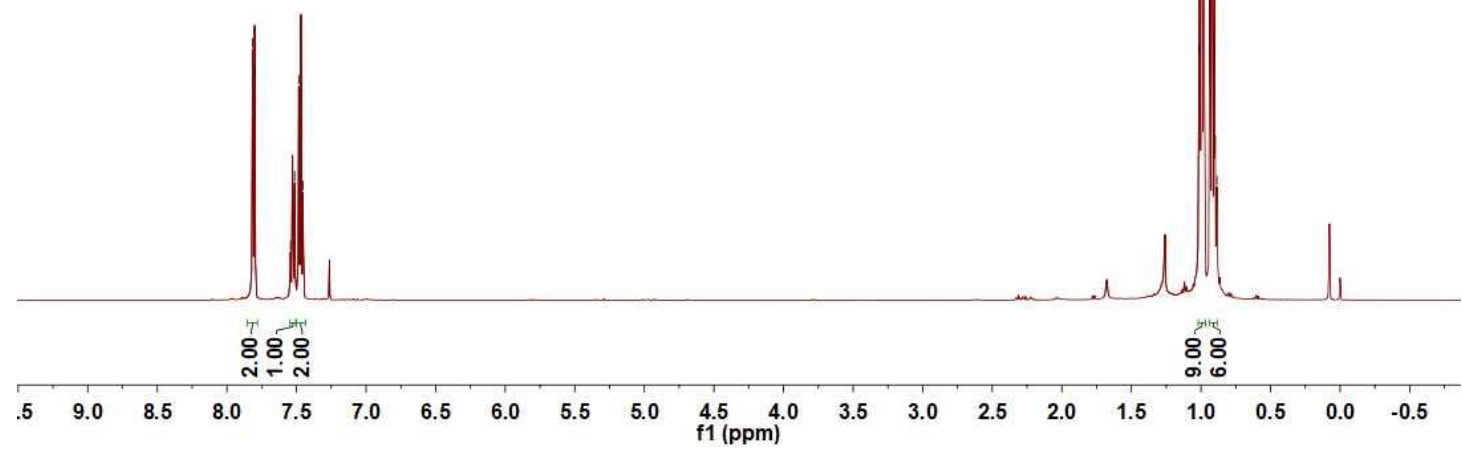

心్లై

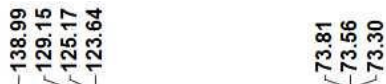

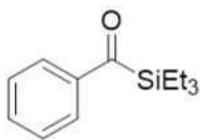

$1 \mathrm{w}$

${ }^{13} \mathrm{C} \mathrm{NMR}\left(\mathrm{CDCl}_{3}, 125 \mathrm{MHz}\right)$

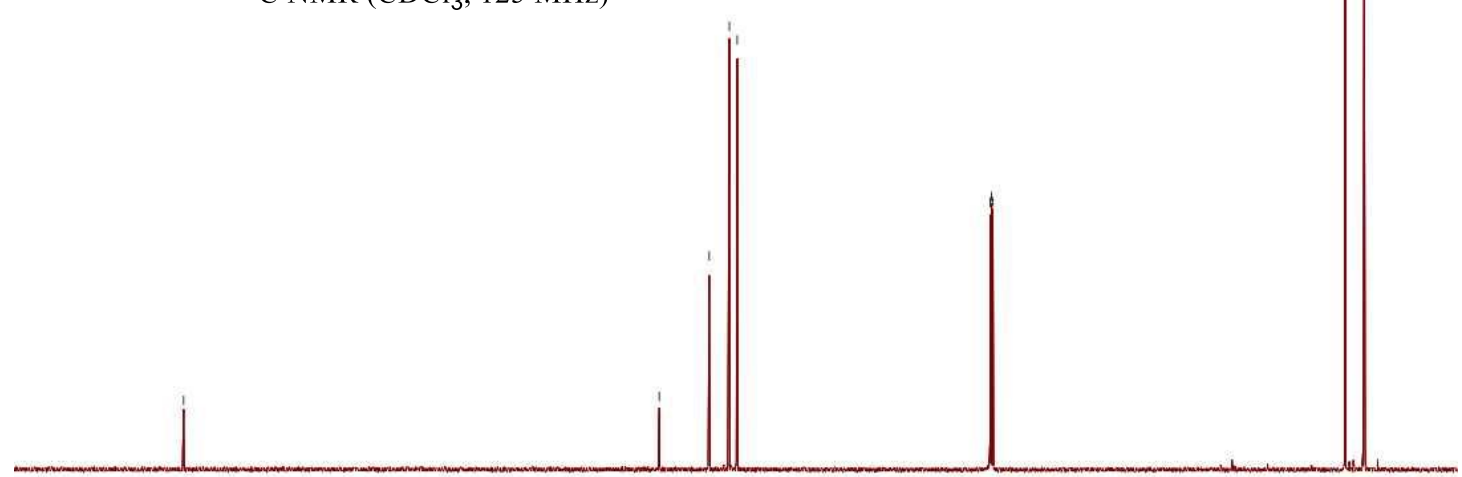

$250 \quad 230$

$\begin{array}{lllll}210 & 190 & 170 & 150 & 130 \\ \mathrm{f} 1(\mathrm{ppm}) & 110\end{array}$

$\begin{array}{lllllllll}90 & 80 & 70 & 60 & 50 & 40 & 30 & 20 & 10\end{array}$ 


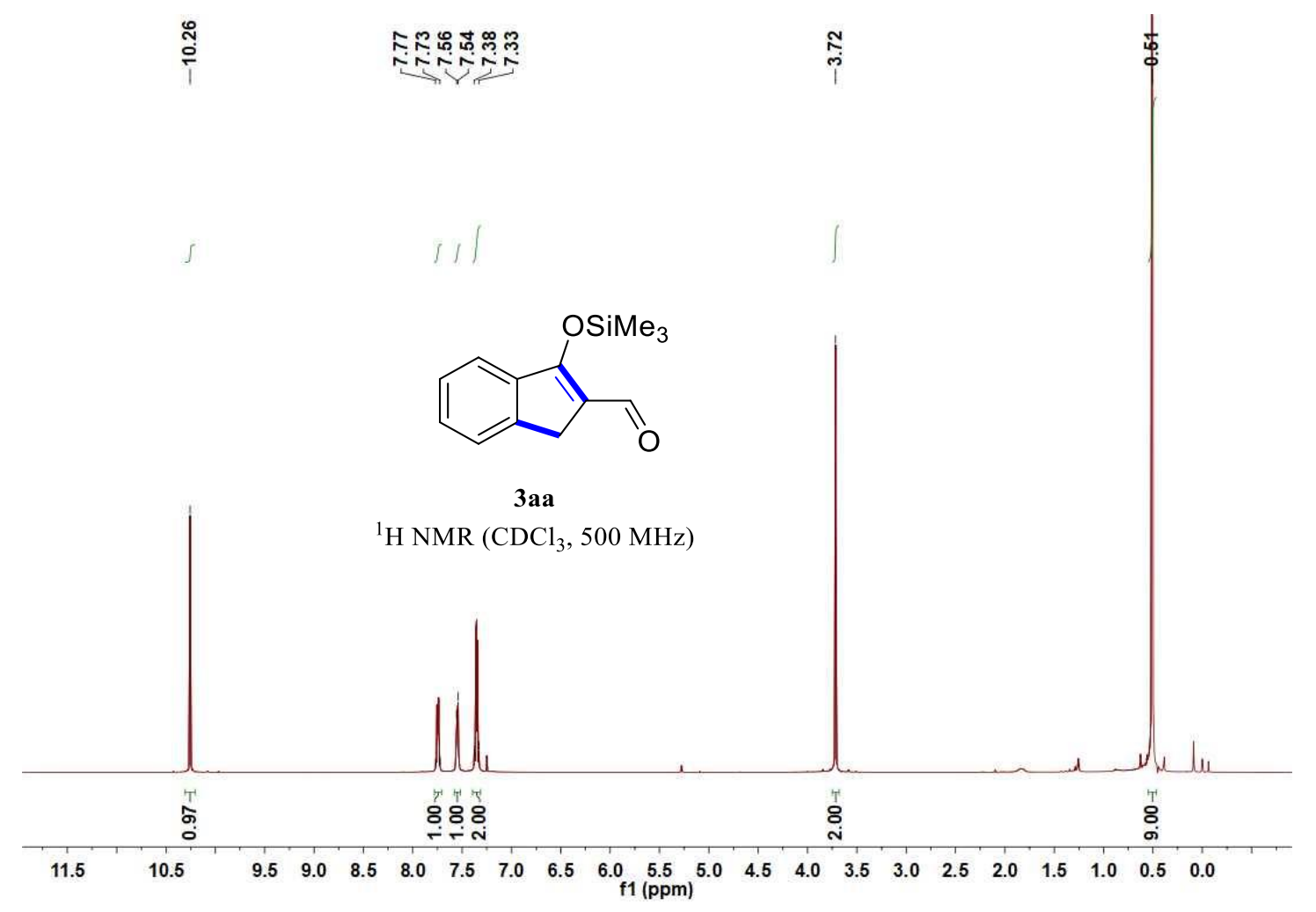

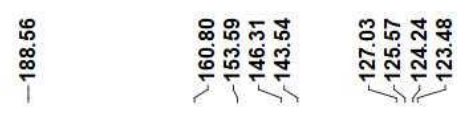

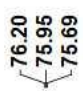

กั

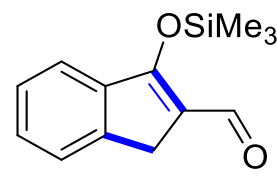

3aa

${ }^{13} \mathrm{C} \mathrm{NMR}\left(\mathrm{CDCl}_{3}, 125 \mathrm{MHz}\right)$

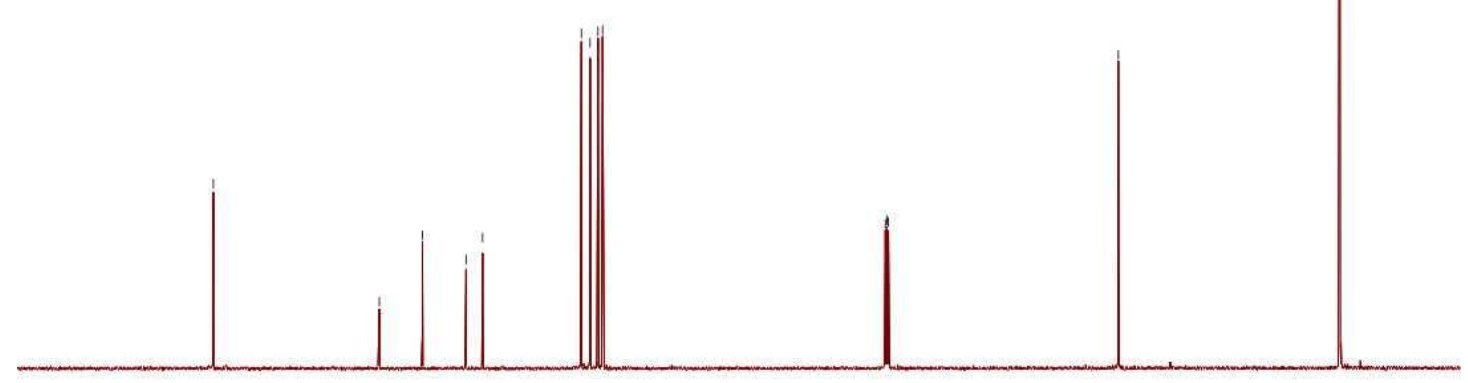

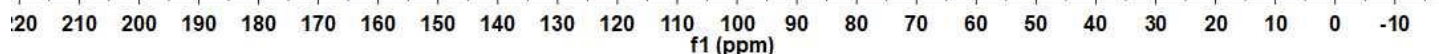




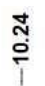

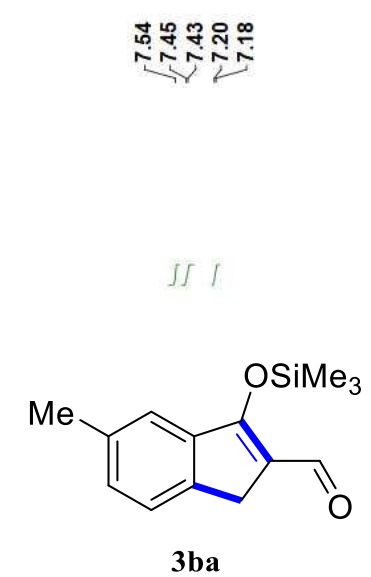

${ }^{1} \mathrm{H}$ NMR $\left(\mathrm{CDCl}_{3}, 500 \mathrm{MHz}\right)$

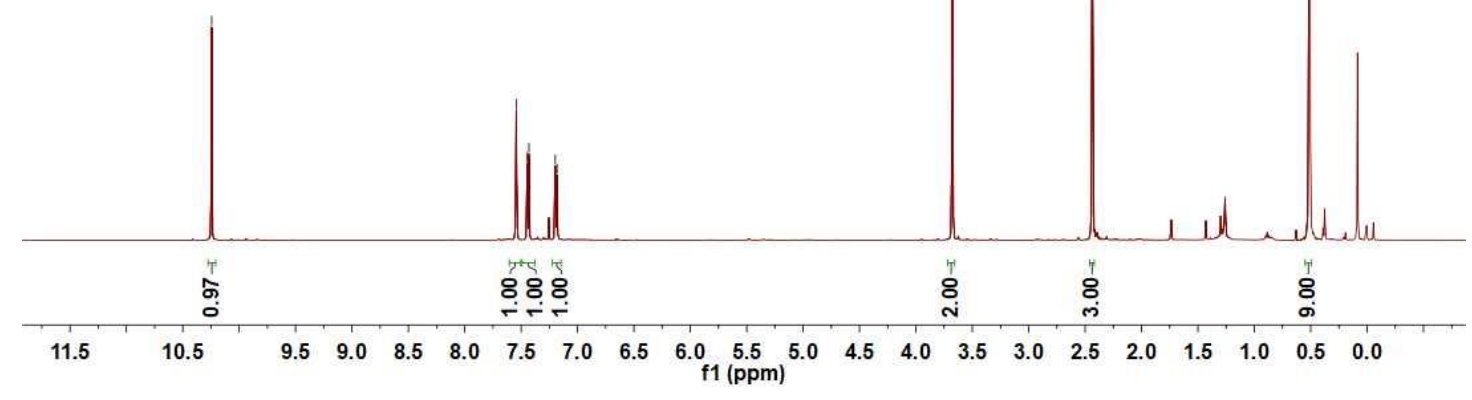

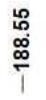

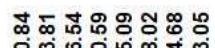

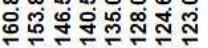

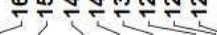

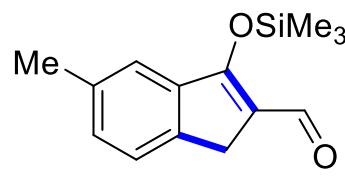

3 ba

吕 声

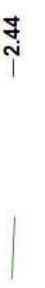

虫

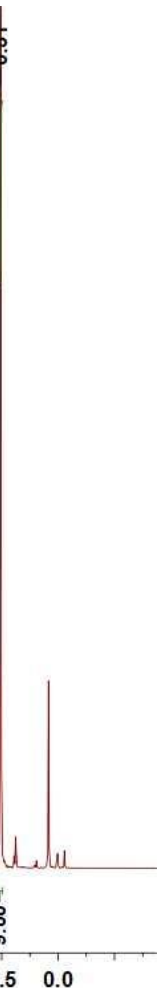

궁ำ

गั0

${ }^{13} \mathrm{C}$ NMR $\left(\mathrm{CDCl}_{3}, 125 \mathrm{MHz}\right)$

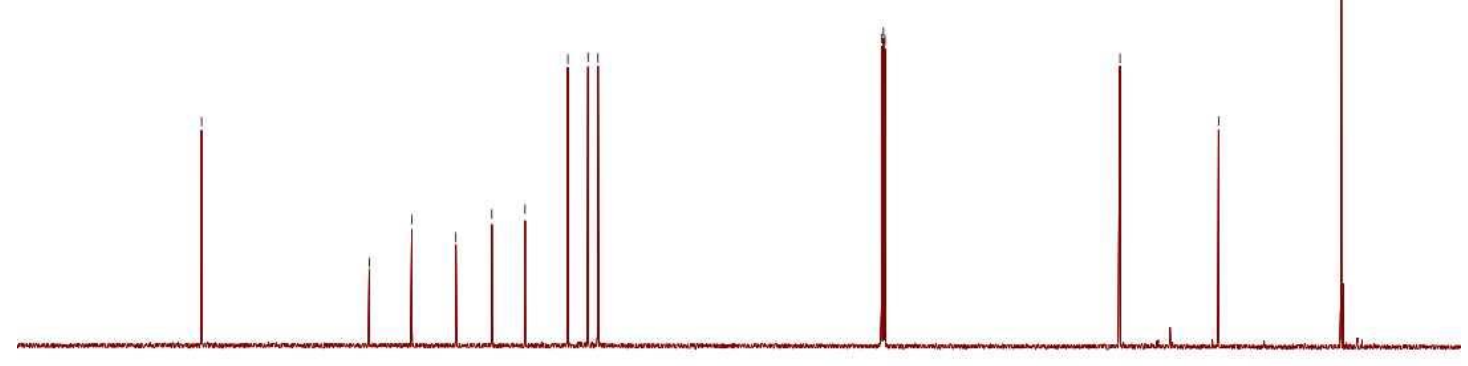

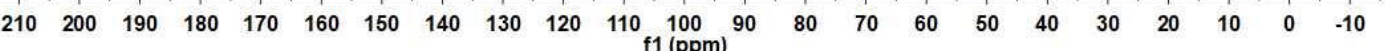




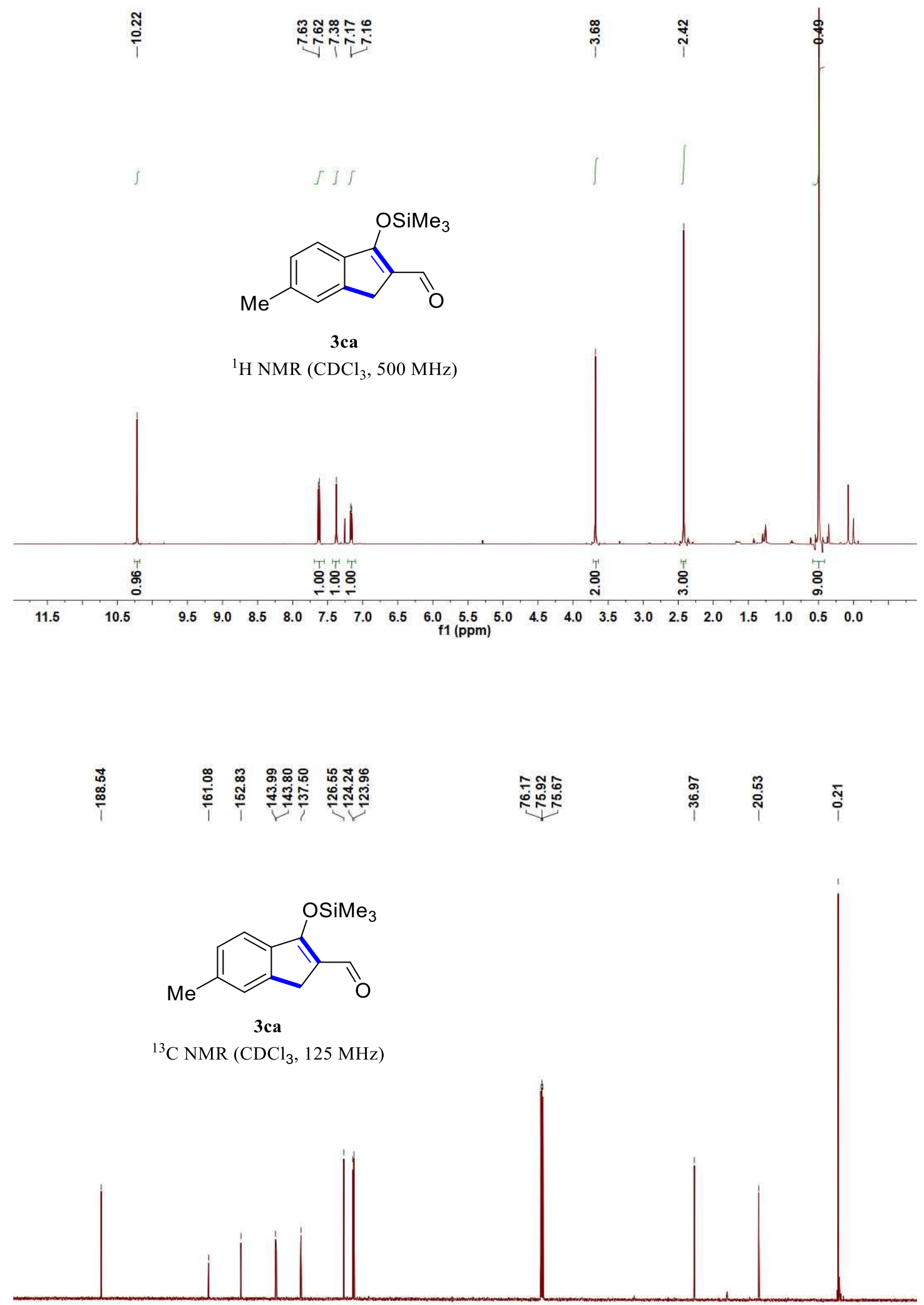

$\begin{array}{llllllllllllllllllllllllllllllllll}10 & 200 & 190 & 180 & 170 & 160 & 150 & 140 & 130 & 120 & 110 & 100 & 90 & 80 & 70 & 60 & 50 & 40 & 30 & 20 & 10 & 0 & -10\end{array}$ 


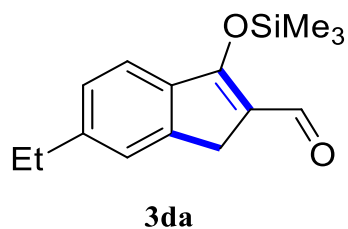

${ }^{1} \mathrm{H}$ NMR $\left(\mathrm{CDCl}_{3}, 500 \mathrm{MHz}\right)$
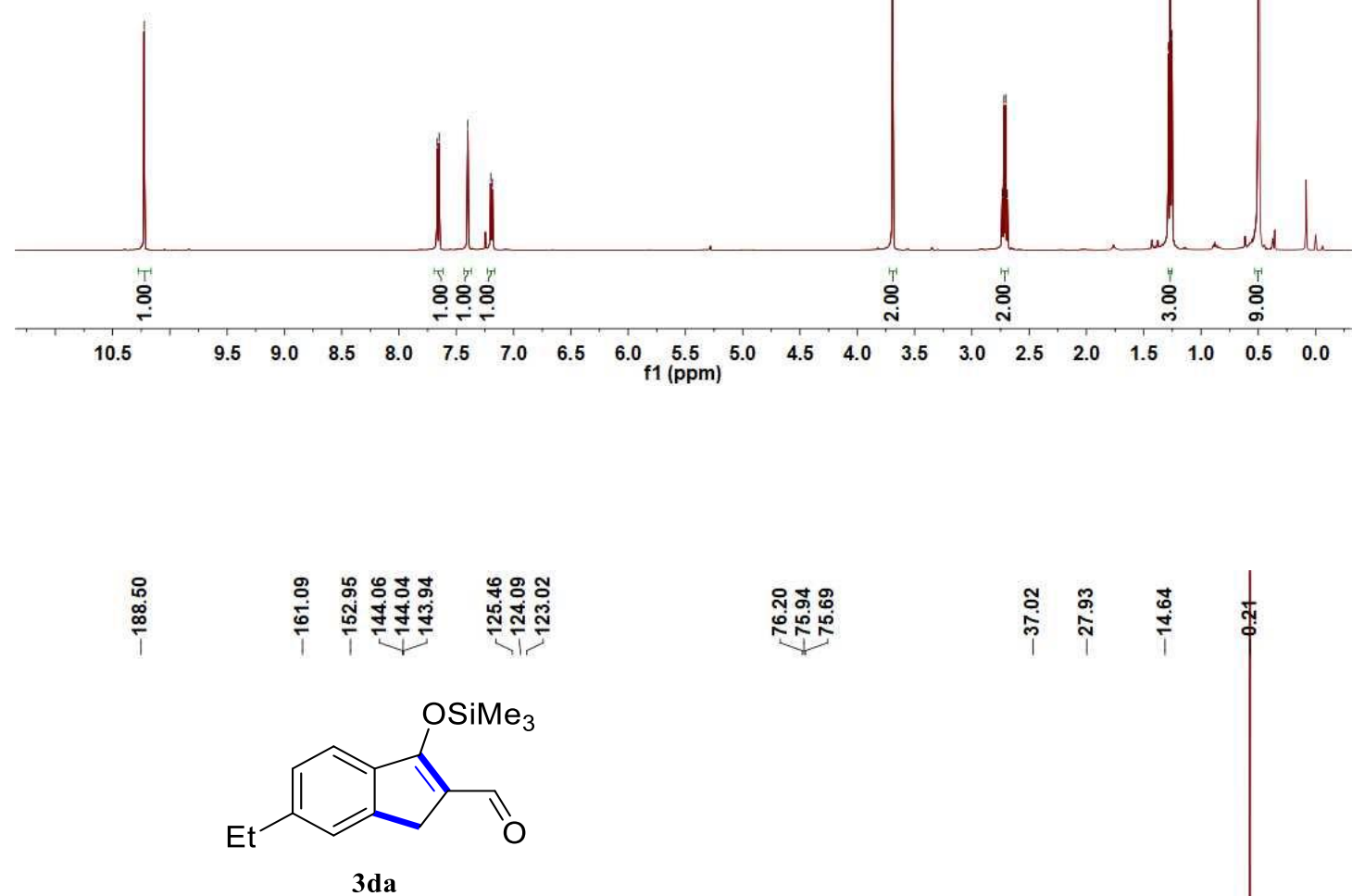

${ }^{13} \mathrm{C} \mathrm{NMR}\left(\mathrm{CDCl}_{3}, 125 \mathrm{MHz}\right)$

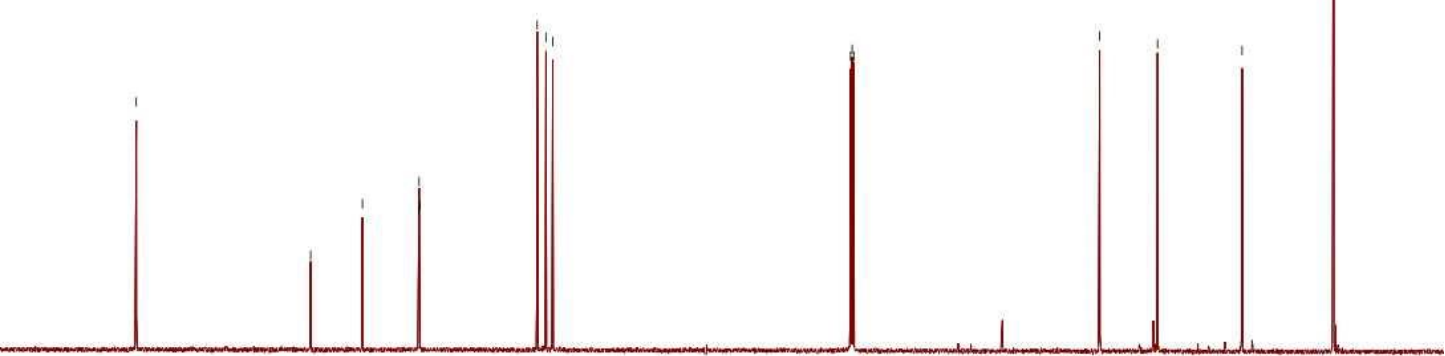

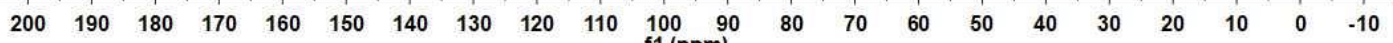



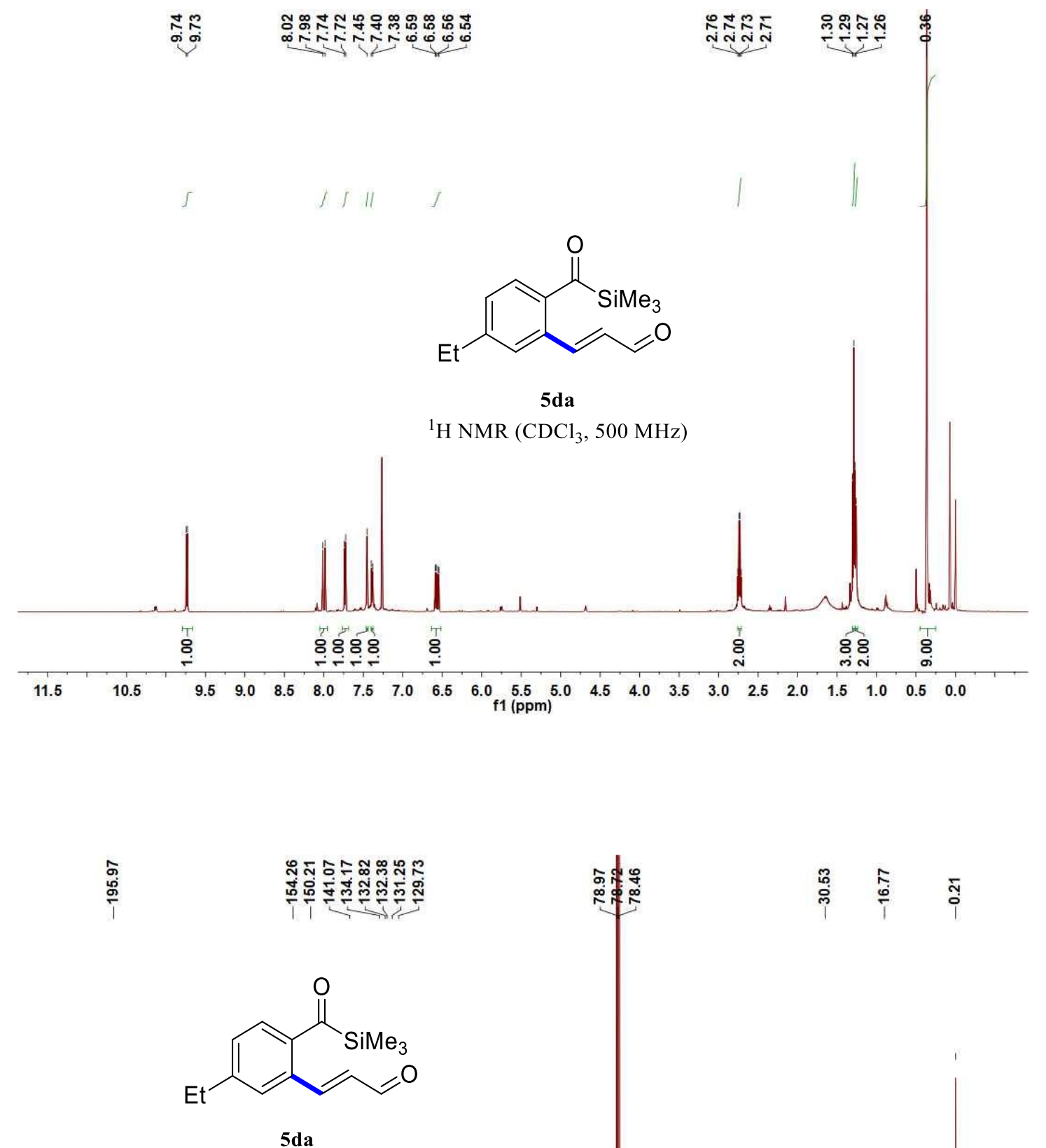

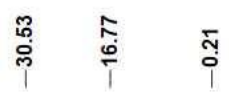

${ }^{13} \mathrm{C} \mathrm{NMR}\left(\mathrm{CDCl}_{3}, 125 \mathrm{MHz}\right)$

$\begin{array}{llllllllllllllllllllll}210 & 200 & 190 & 180 & 170 & 160 & 150 & 140 & 130 & 120 & 110 \begin{array}{l}100 \\ \mathrm{f} 1(\mathrm{ppm})\end{array} & 90 & 80 & 70 & 60 & 50 & 40 & 30 & 20 & 10 & 0 & -10\end{array}$ 


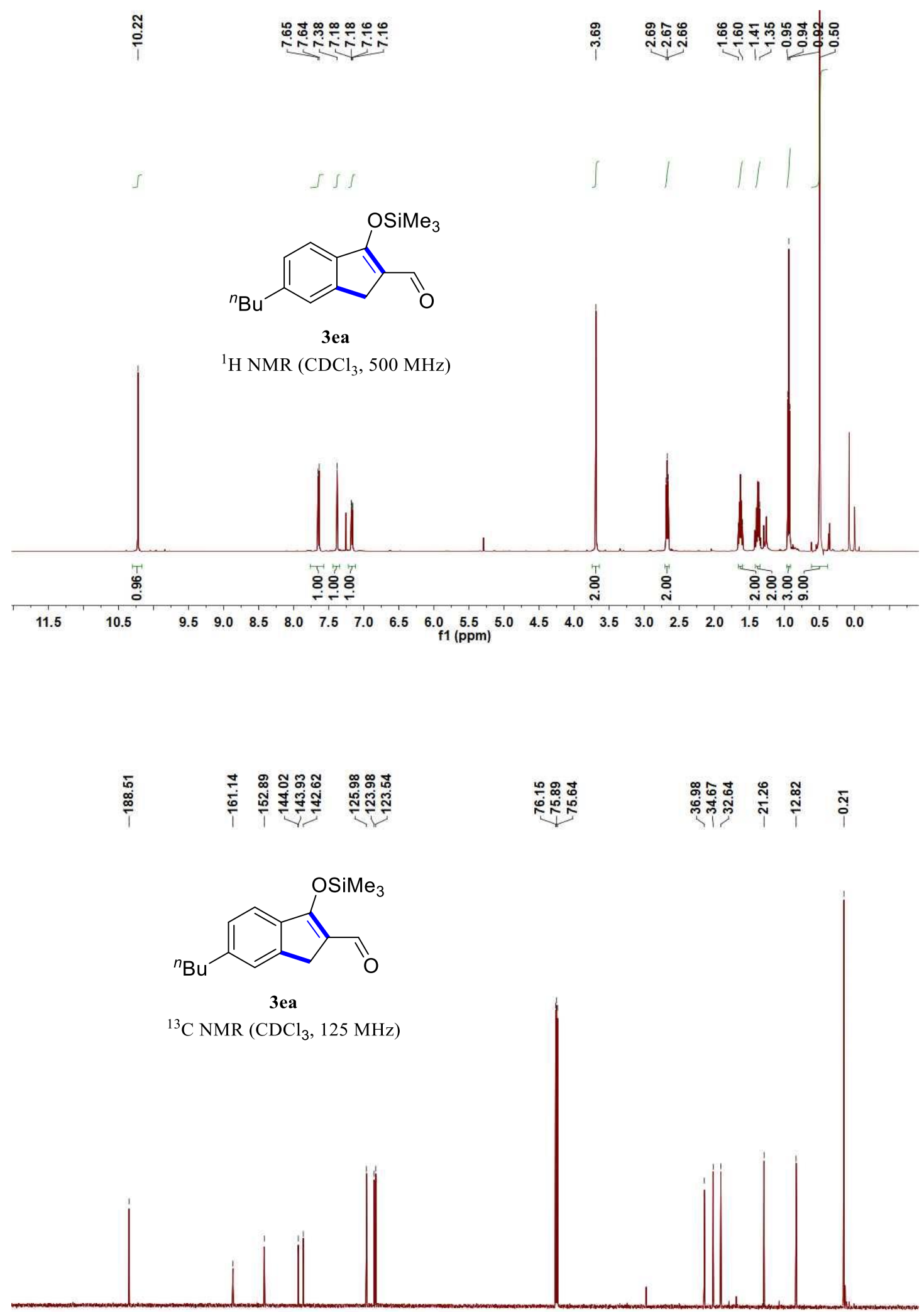

$\begin{array}{llllllllllllllllllllllll}210 & 200 & 190 & 180 & 170 & 160 & 150 & 140 & 130 & 120 & 110 & \begin{array}{l}100 \\ \mathrm{f} 1(\mathrm{ppm})\end{array} & 90 & 80 & 70 & 60 & 50 & 40 & 30 & 20 & 10 & 0 & -10\end{array}$ 


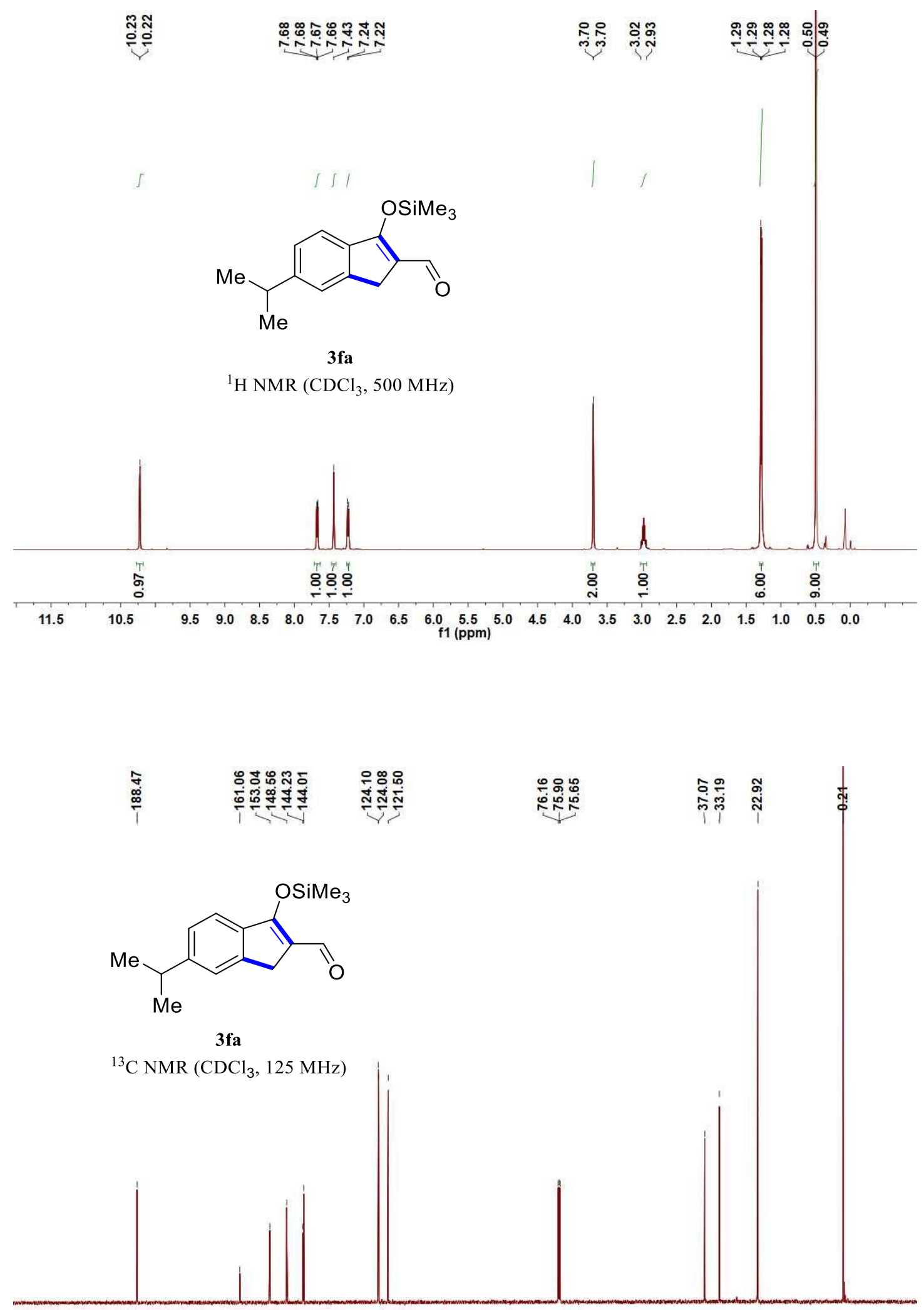

$\begin{array}{llllllllllllllllllllllllllll}20 & 210 & 200 & 190 & 180 & 170 & 160 & 150 & 140 & 130 & 120 & 110 & 100 & 90 & 80 & 70 & 60 & 50 & 40 & 30 & 20 & 10 & 0 & -10\end{array}$ 

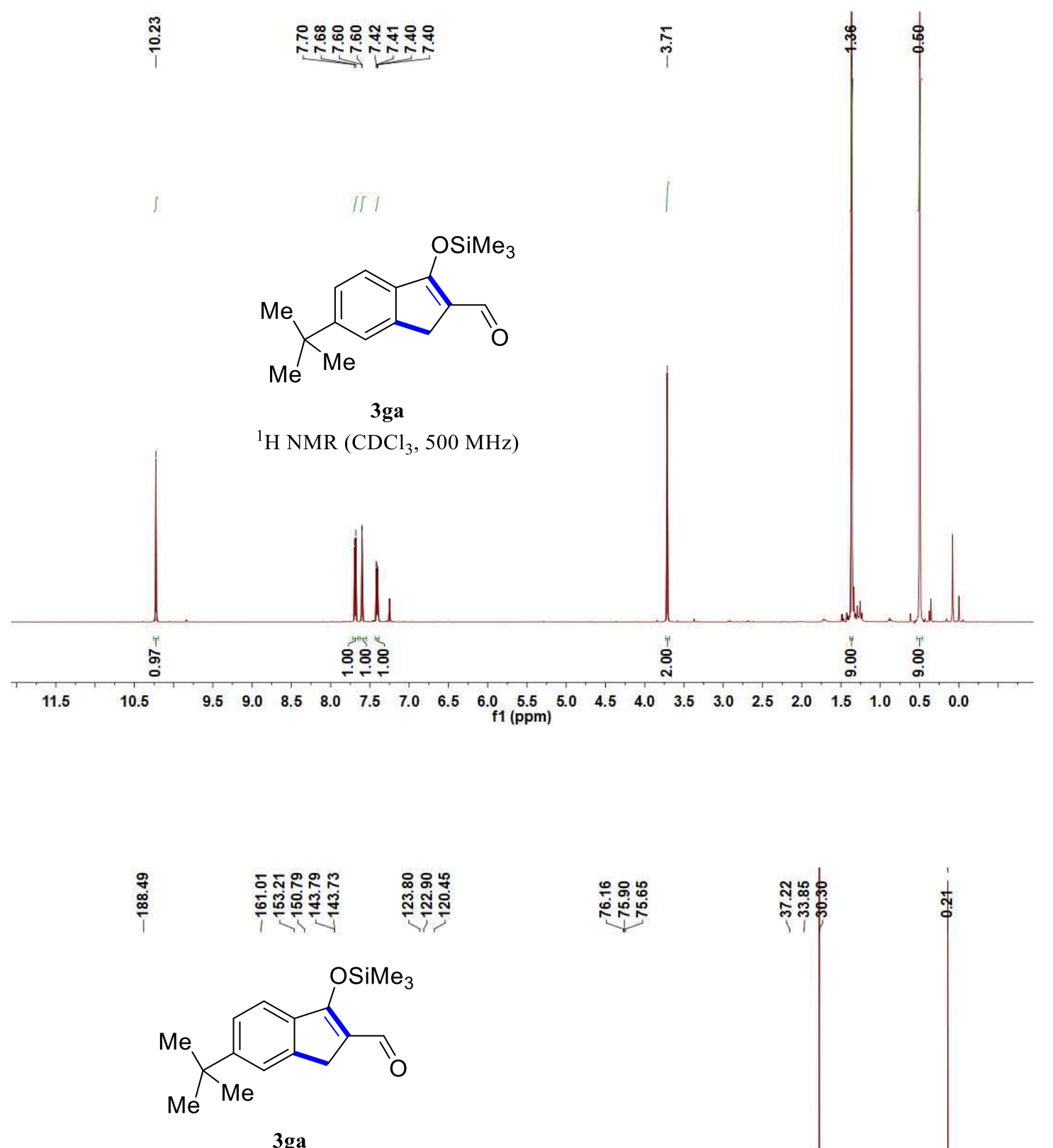

${ }^{13} \mathrm{C} \mathrm{NMR}\left(\mathrm{CDCl}_{3}, 125 \mathrm{MHz}\right)$

$\begin{array}{lllllllllllllllllllllll}210 & 200 & 190 & 180 & 170 & 160 & 150 & 140 & 130 & 120 & 110 & 100 & 90 & 80 & 70 & 60 & 50 & 40 & 30 & 20 & 10 & 0 & -10\end{array}$ 


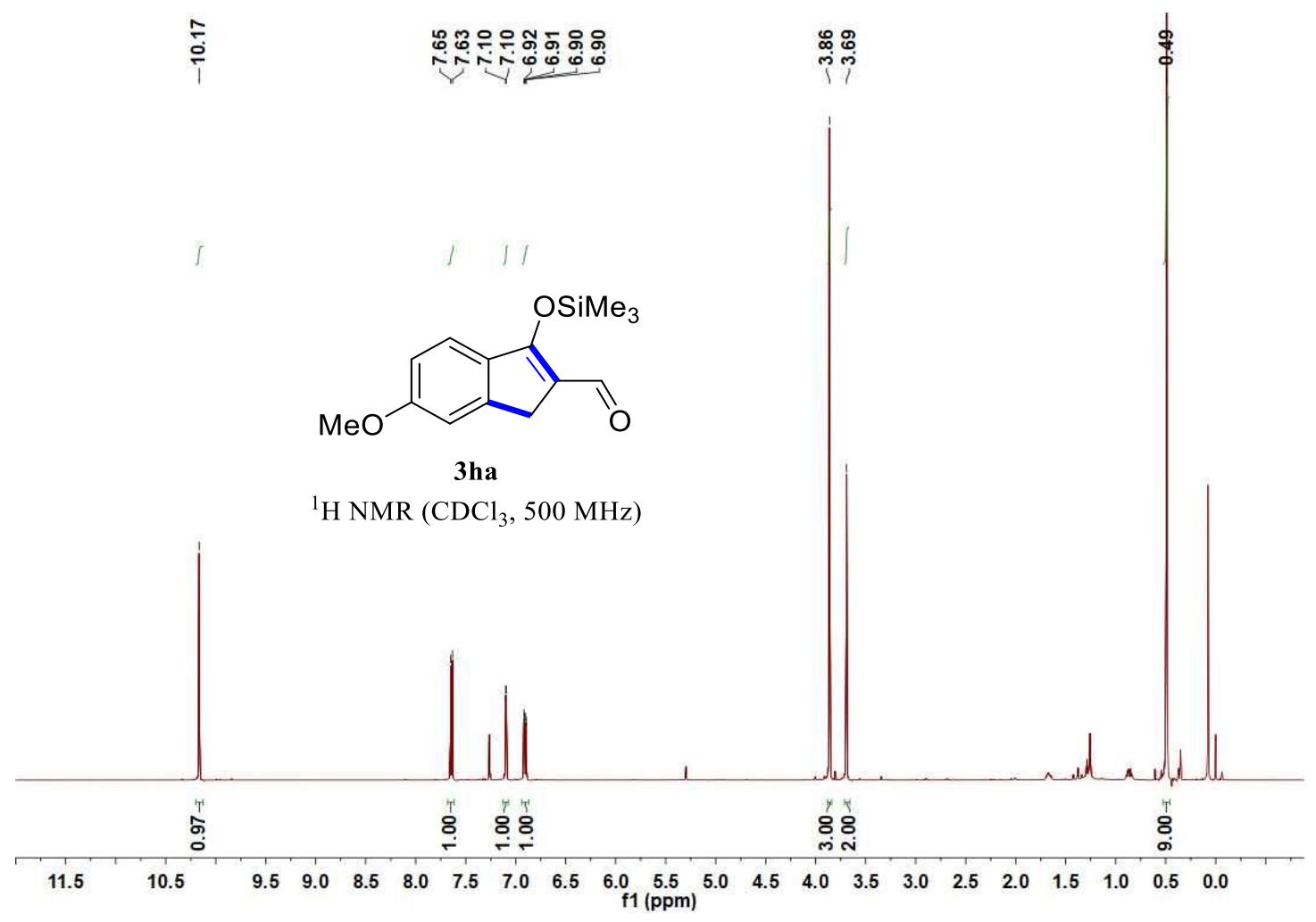

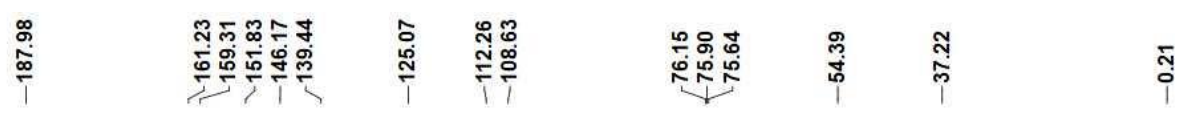

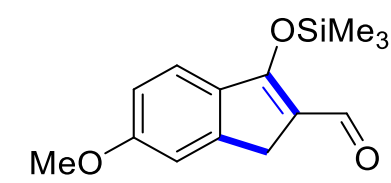

3ha

${ }^{13} \mathrm{C} \mathrm{NMR}\left(\mathrm{CDCl}_{3}, 125 \mathrm{MHz}\right)$

$\begin{array}{lllllllllllllllllllllll}210 & 200 & 190 & 180 & 170 & 160 & 150 & 140 & 130 & 120 & 110 & 100 & 90 & 80 & 70 & 60 & 50 & 40 & 30 & 20 & 10 & 0 & -10\end{array}$ 

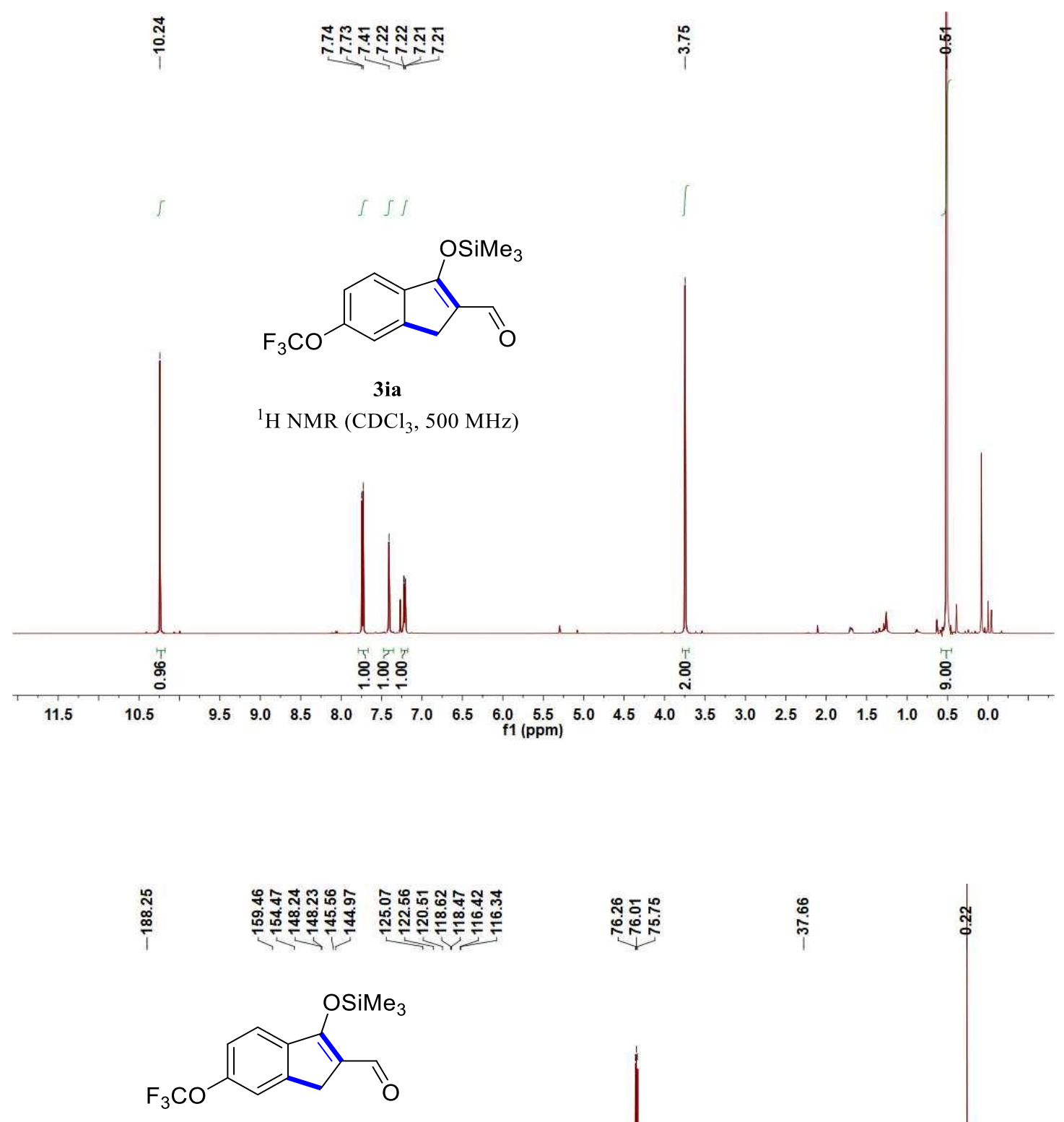

3ia

${ }^{13} \mathrm{C} \mathrm{NMR}\left(\mathrm{CDCl}_{3}, 125 \mathrm{MHz}\right)$

$\begin{array}{llllllllllllllllllllll}210 & 200 & 190 & 180 & 170 & 160 & 150 & 140 & 130 & 120 & 110 \begin{array}{l}100 \\ \mathrm{f} 1(\mathrm{ppm})\end{array} & 90 & 80 & 70 & 60 & 50 & 40 & 30 & 20 & 10 & 0 & -10\end{array}$ 

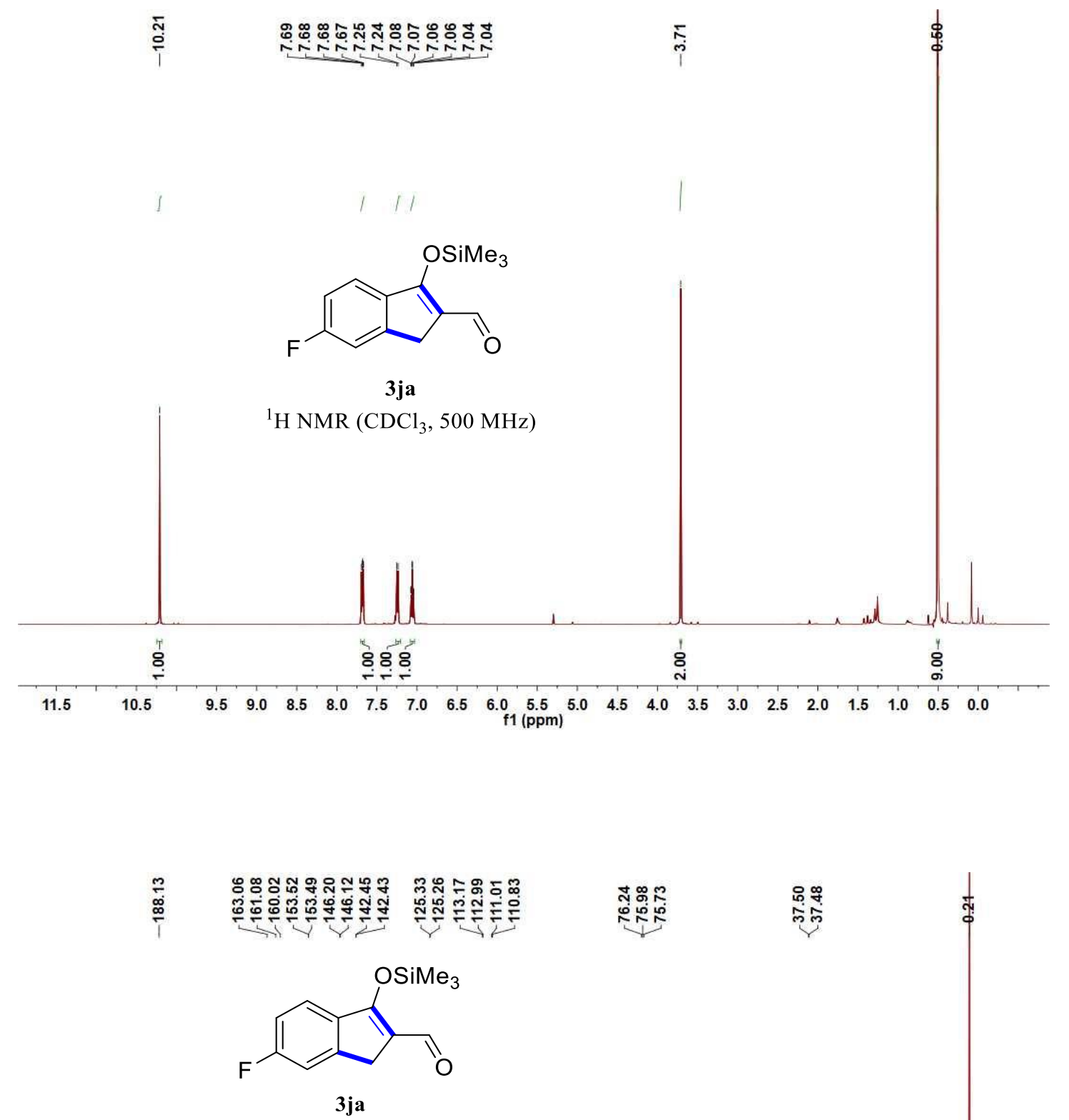

${ }^{13} \mathrm{C} \mathrm{NMR}\left(\mathrm{CDCl}_{3}, 125 \mathrm{MHz}\right)$

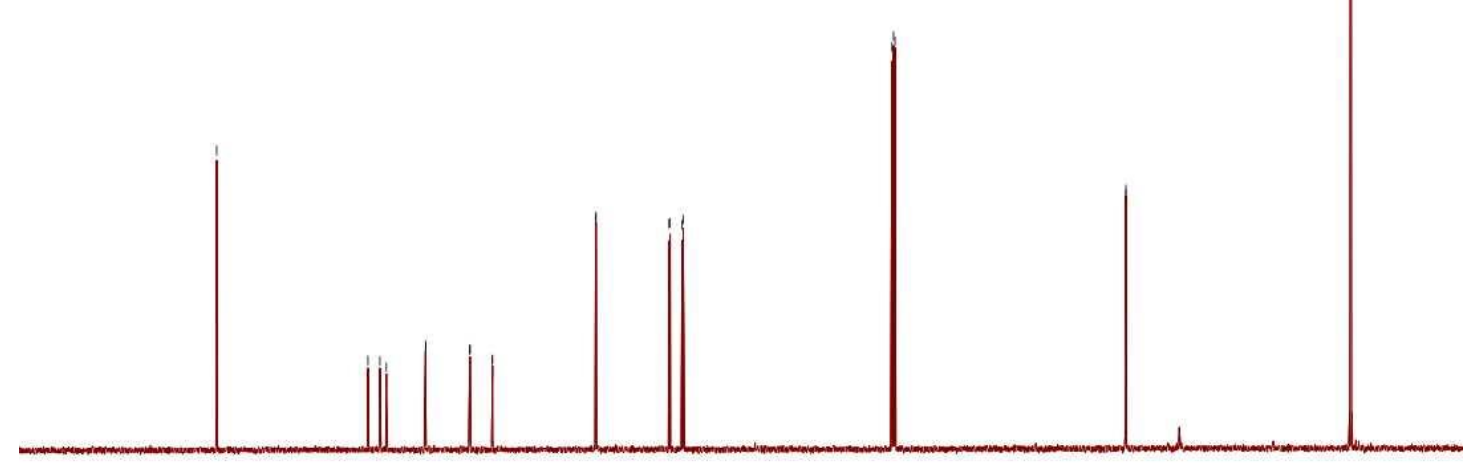

$\begin{array}{lllllllllllllllllllllllllllllll}20 & 210 & 200 & 190 & 180 & 170 & 160 & 150 & 140 & 130 & 120 & 110 & 100 & 90 & 80 & 70 & 60 & 50 & 40 & 30 & 20 & 10 & 0 & -10\end{array}$ 

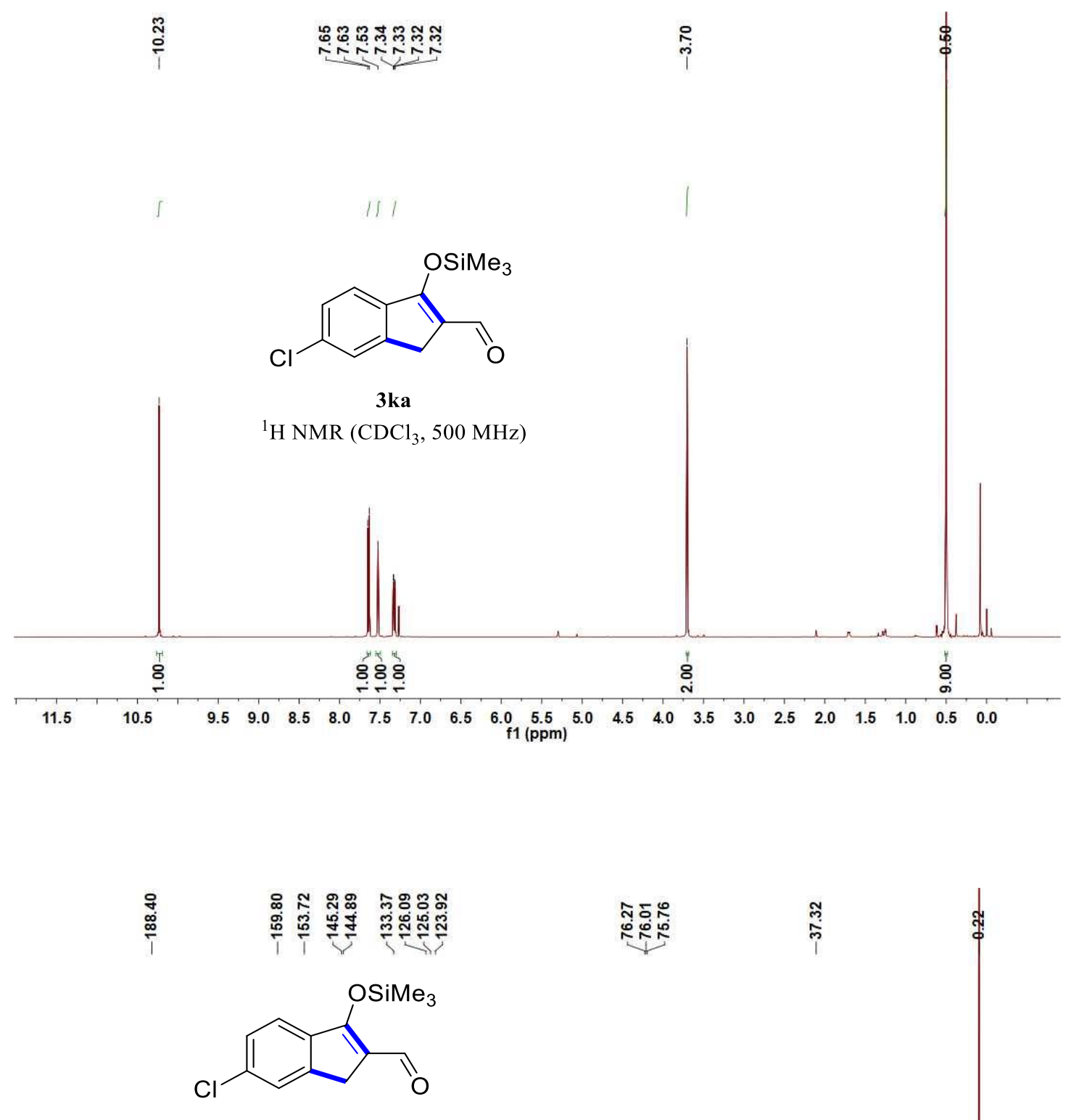

$3 \mathbf{k a}$

${ }^{13} \mathrm{C} \mathrm{NMR}\left(\mathrm{CDCl}_{3}, 125 \mathrm{MHz}\right)$

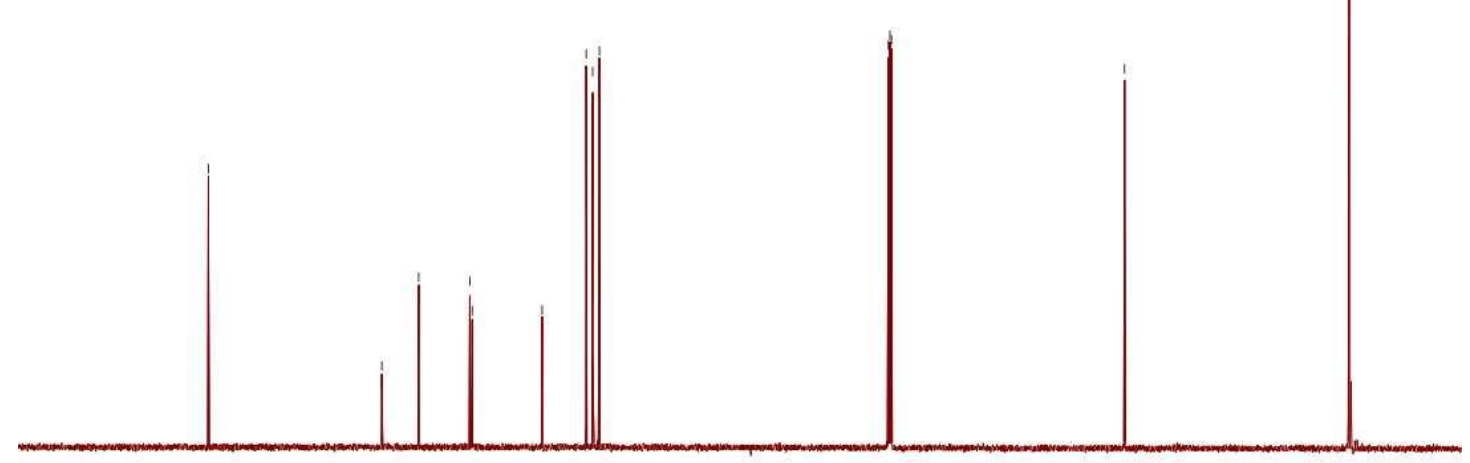

$\begin{array}{llllllllllllllllllllll}210 & 200 & 190 & 180 & 170 & 160 & 150 & 140 & 130 & 120 & 110 \begin{array}{l}100 \\ \mathrm{f} 1(\mathrm{ppm})\end{array} & 90 & 80 & 70 & 60 & 50 & 40 & 30 & 20 & 10 & 0 & -10\end{array}$ 


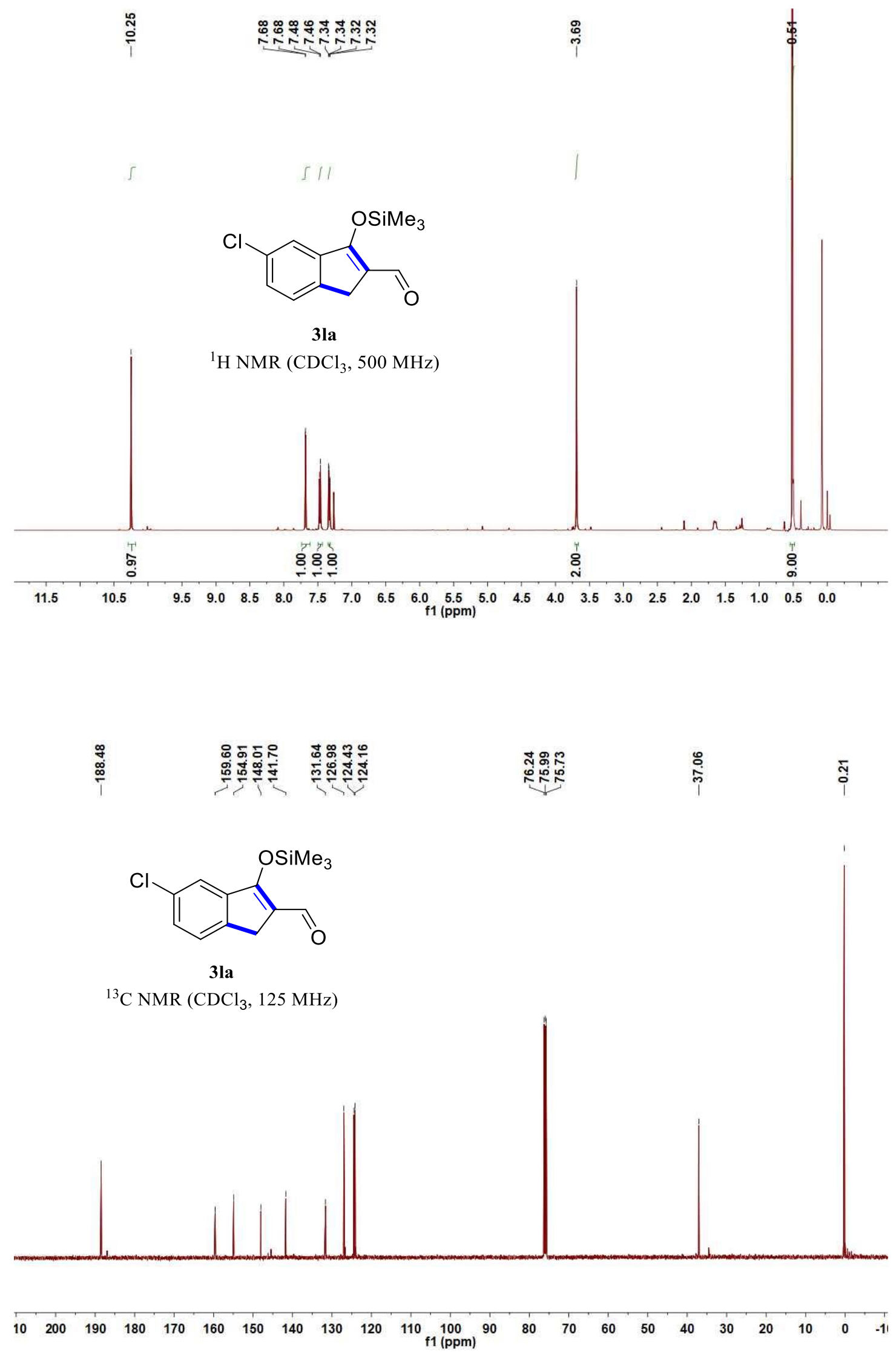



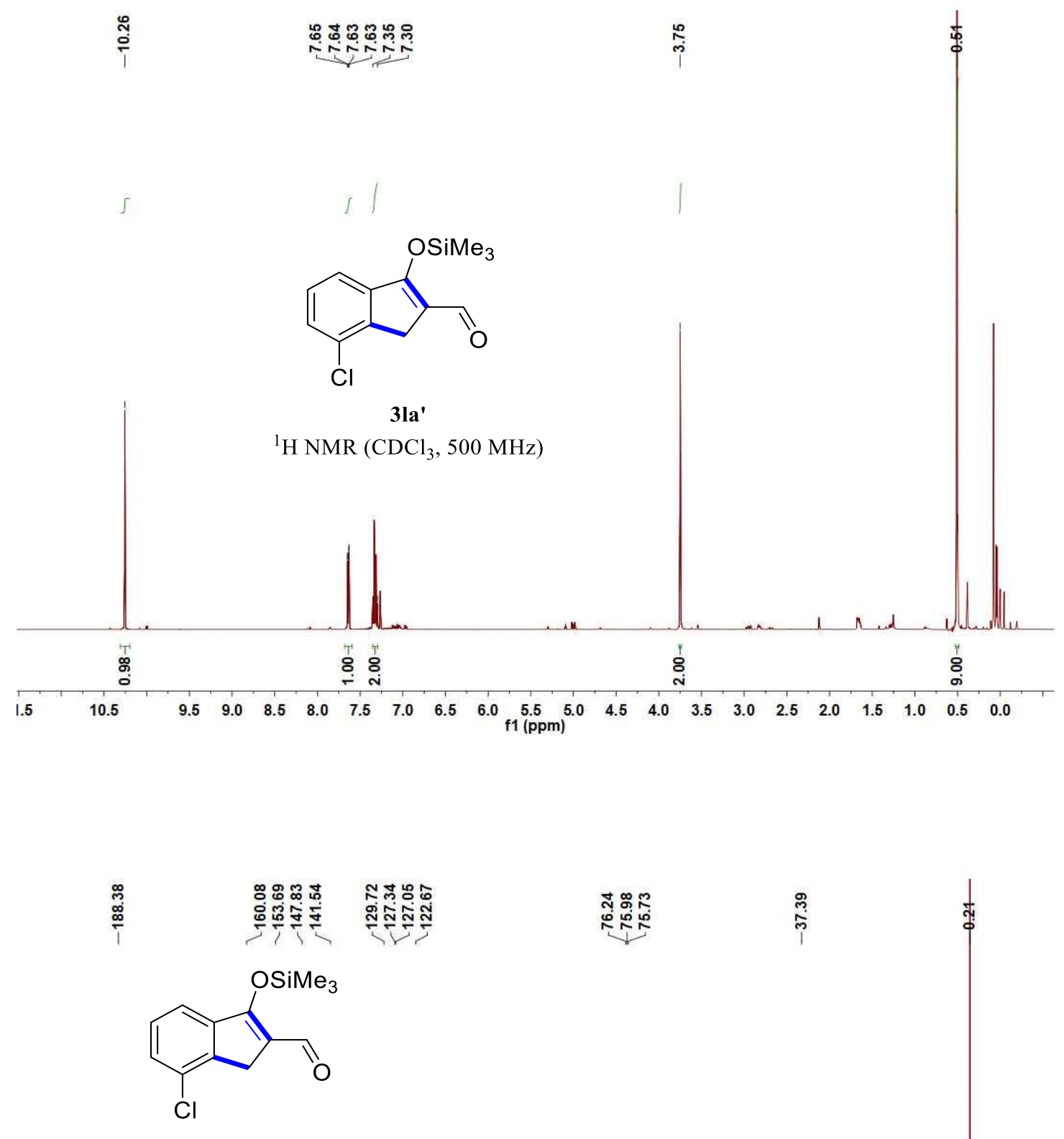

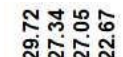

끄는

సุำ

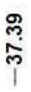

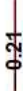

3la'

${ }^{13} \mathrm{C} \mathrm{NMR}\left(\mathrm{CDCl}_{3}, 125 \mathrm{MHz}\right)$

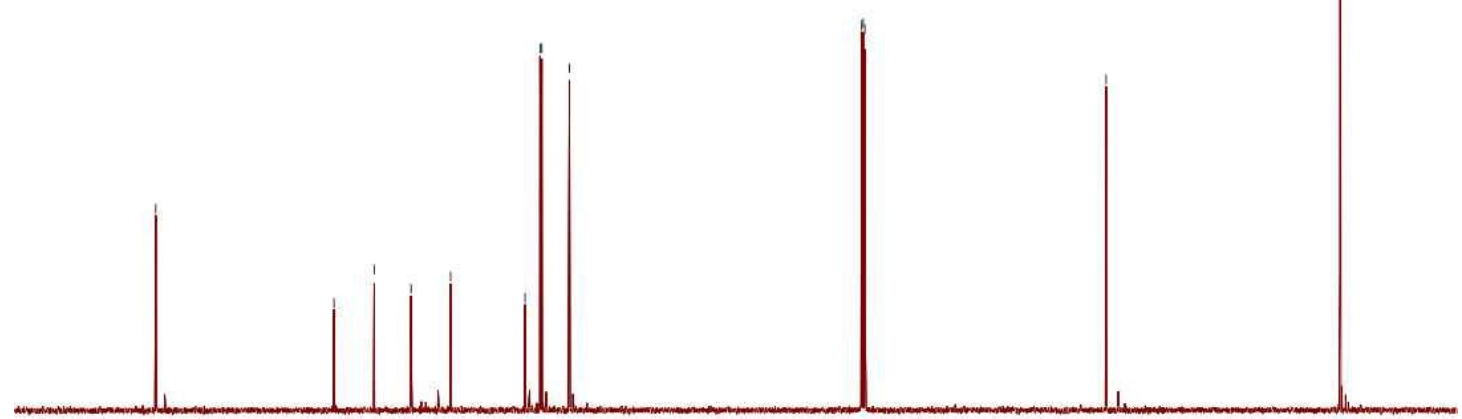

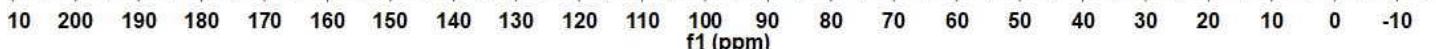




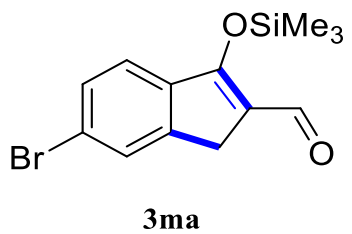

${ }^{1} \mathrm{H} \mathrm{NMR}\left(\mathrm{CDCl}_{3}, 500 \mathrm{MHz}\right)$

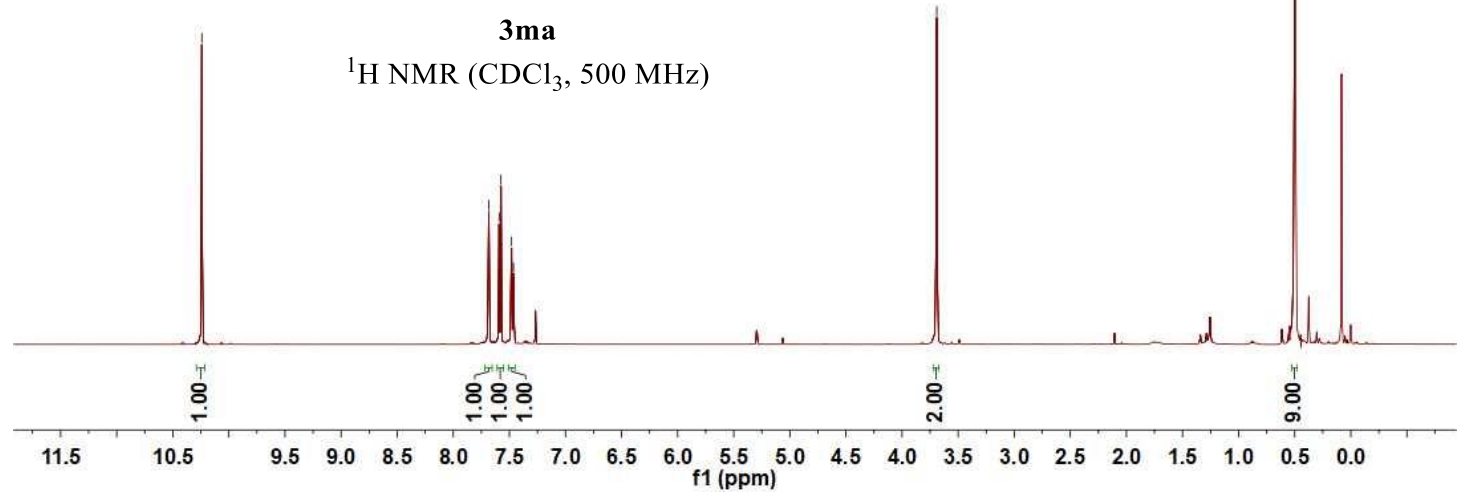

倠

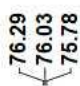

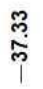<smiles>COC1=C(C=O)Cc2cc(Br)ccc21</smiles>

$3 \mathrm{ma}$

${ }^{13} \mathrm{C} \mathrm{NMR}\left(\mathrm{CDCl}_{3}, 125 \mathrm{MHz}\right)$

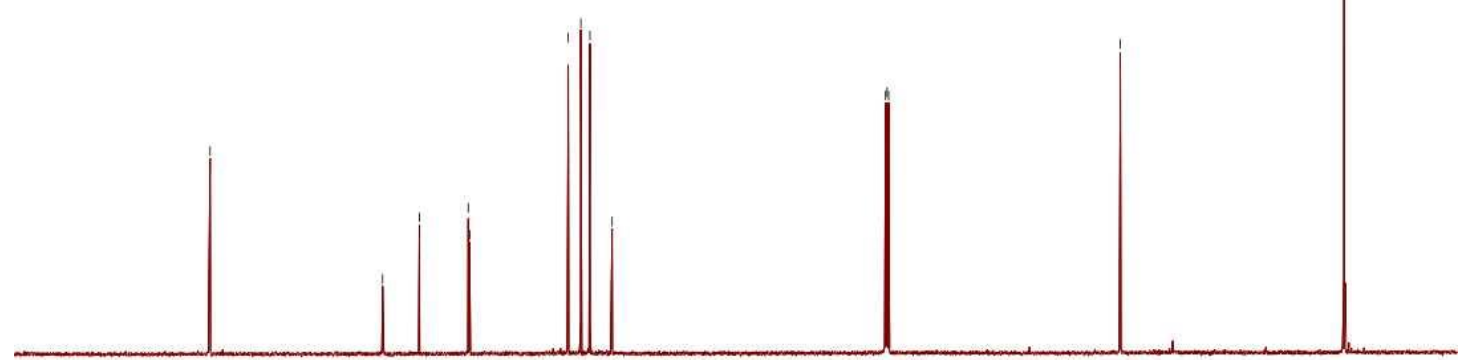

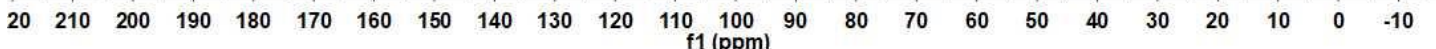




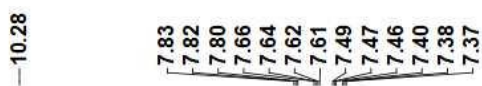

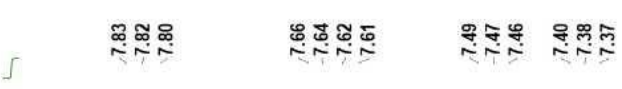

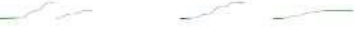

${ }^{1} \mathrm{H}$ NMR $\left(\mathrm{CDCl}_{3}, 500 \mathrm{MHz}\right)$

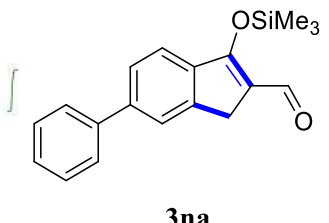

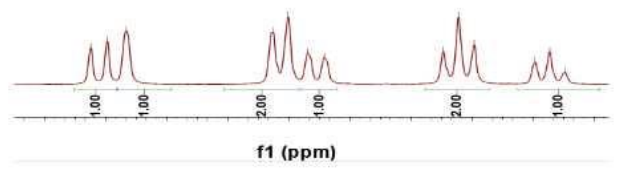
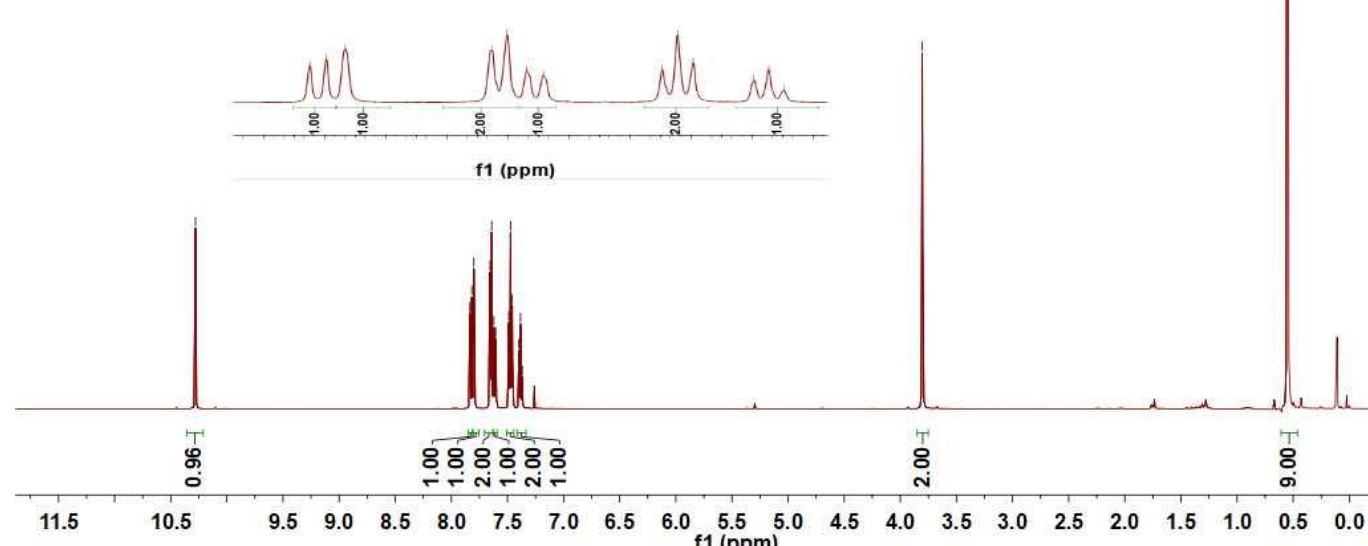

$\stackrel{8}{\mathrm{i}}$ के

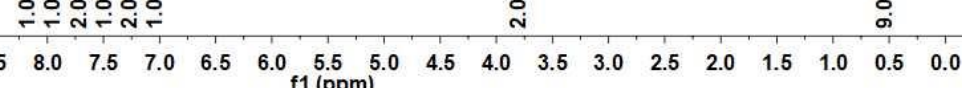

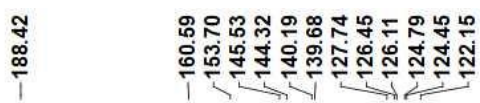
皆㖞

กุ

is
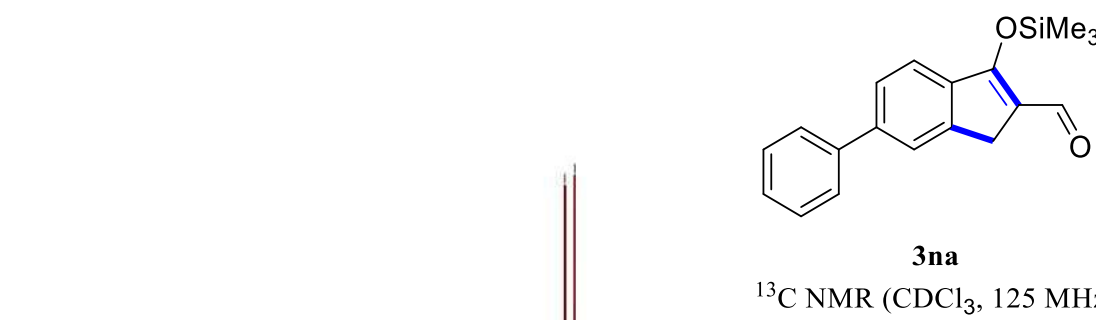

3 a

${ }^{13} \mathrm{C}$ NMR $\left(\mathrm{CDCl}_{3}, 125 \mathrm{MHz}\right)$

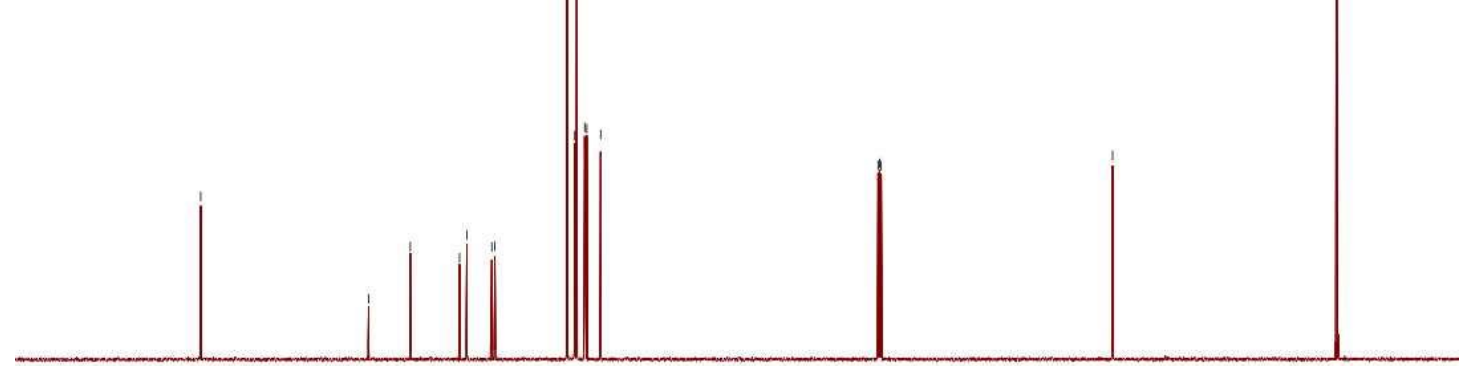

$\begin{array}{llllllllllllllllllllll}210 & 200 & 190 & 180 & 170 & 160 & 150 & 140 & 130 & 120 & 110 \begin{array}{l}100 \\ \mathrm{f} 1(\mathrm{ppm})\end{array} & 90 & 80 & 70 & 60 & 50 & 40 & 30 & 20 & 10 & 0 & -10\end{array}$ 


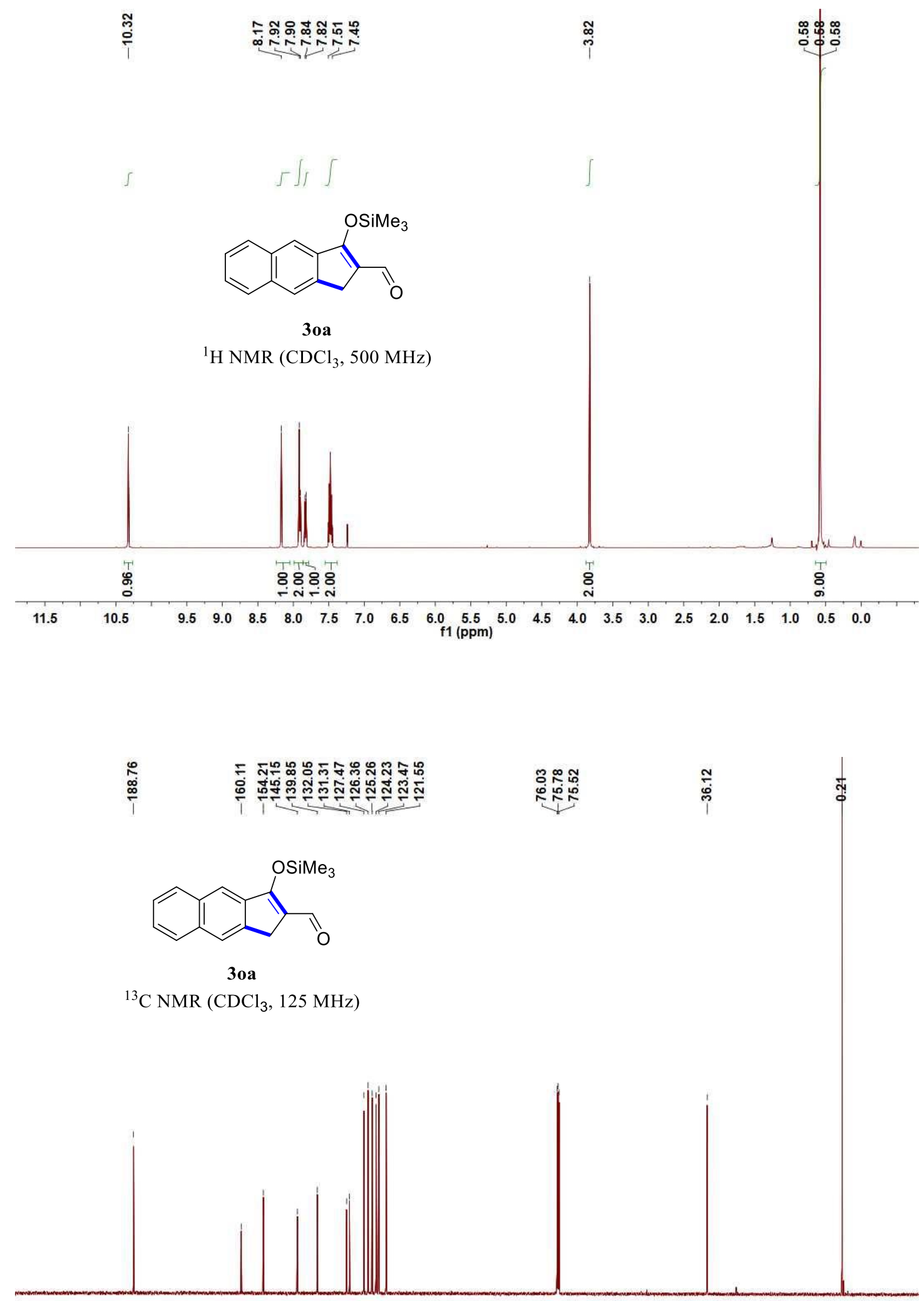

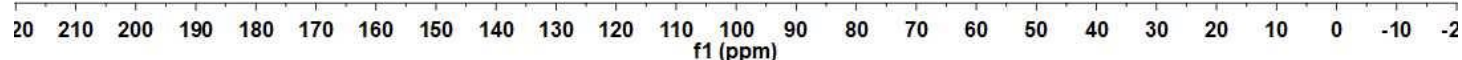


$\mathrm{Me}$
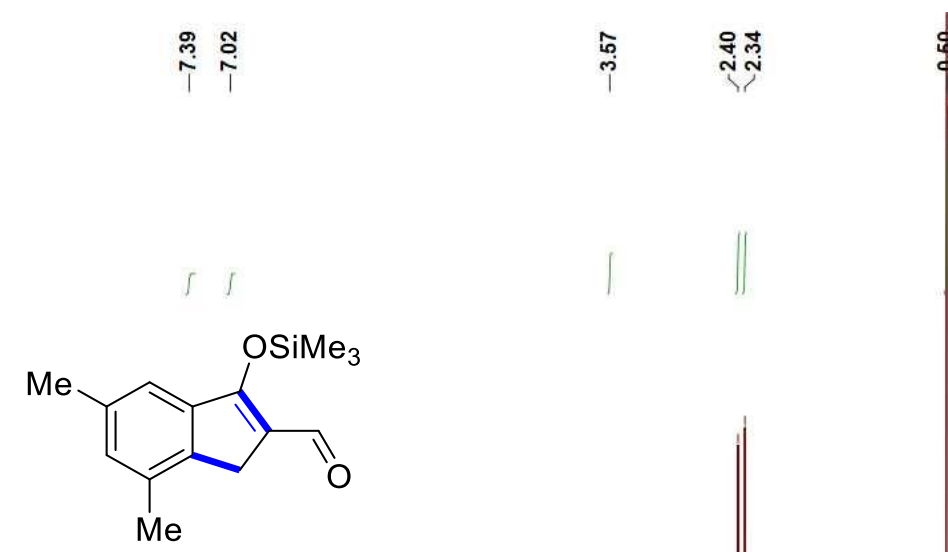

3pa

${ }^{1} \mathrm{H} \mathrm{NMR}\left(\mathrm{CDCl}_{3}, 500 \mathrm{MHz}\right)$
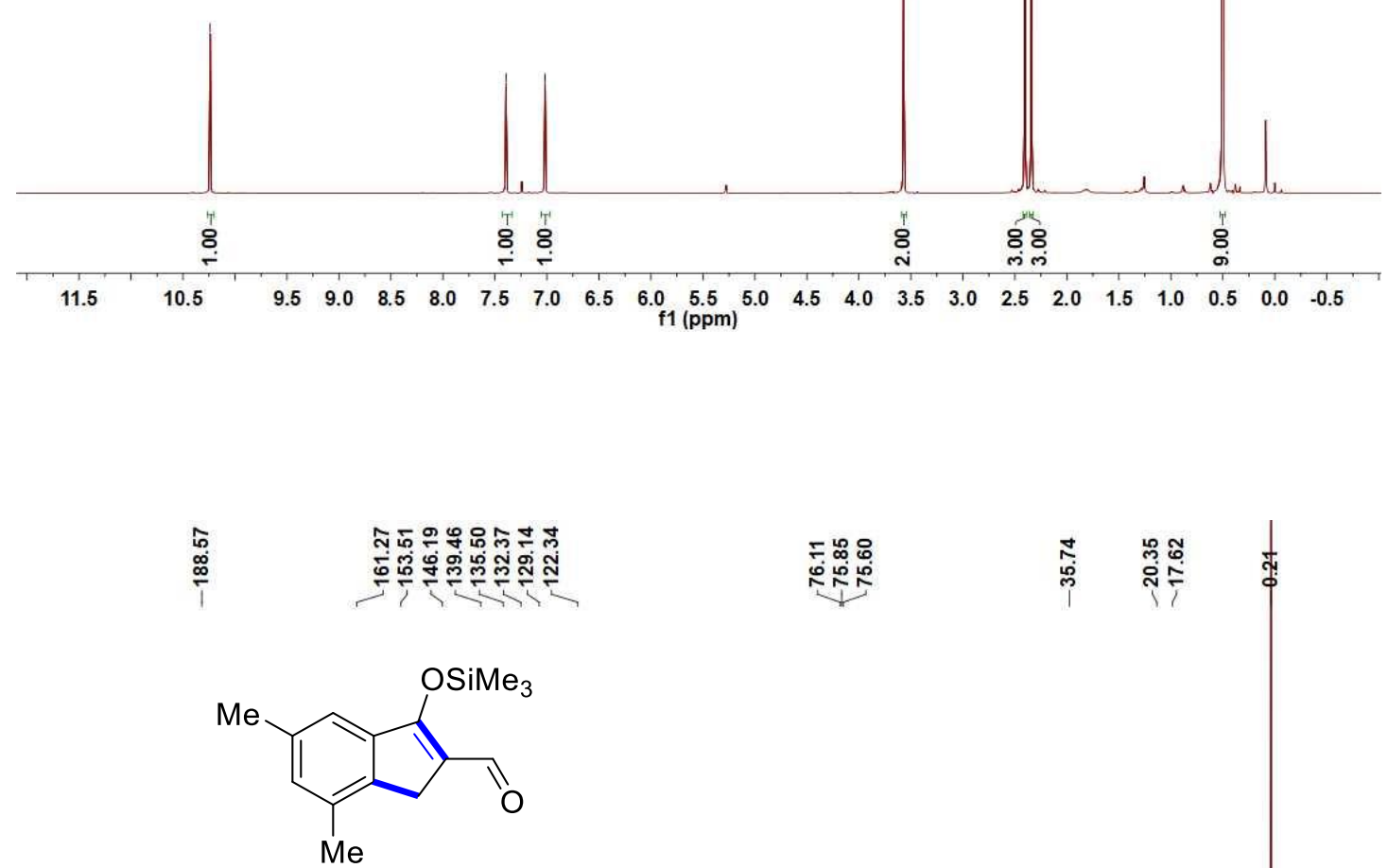

3 pa

${ }^{13} \mathrm{C} \mathrm{NMR}\left(\mathrm{CDCl}_{3}, 125 \mathrm{MHz}\right)$

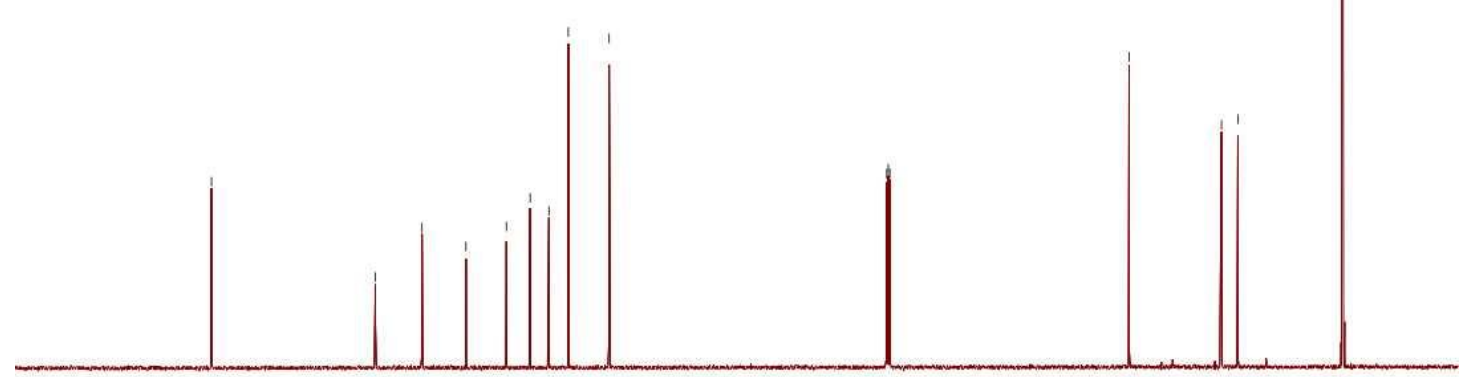

$\begin{array}{lllllllllllllllllllllll}20 & 210 & 200 & 190 & 180 & 170 & 160 & 150 & 140 & 130 & 120 & \begin{array}{c}110 \\ \mathrm{f} 1(\mathrm{ppm})\end{array} & \begin{array}{l}100 \\ \text { (po }\end{array} & 80 & 70 & 60 & 50 & 40 & 30 & 20 & 10 & 0 & -10\end{array}$ 


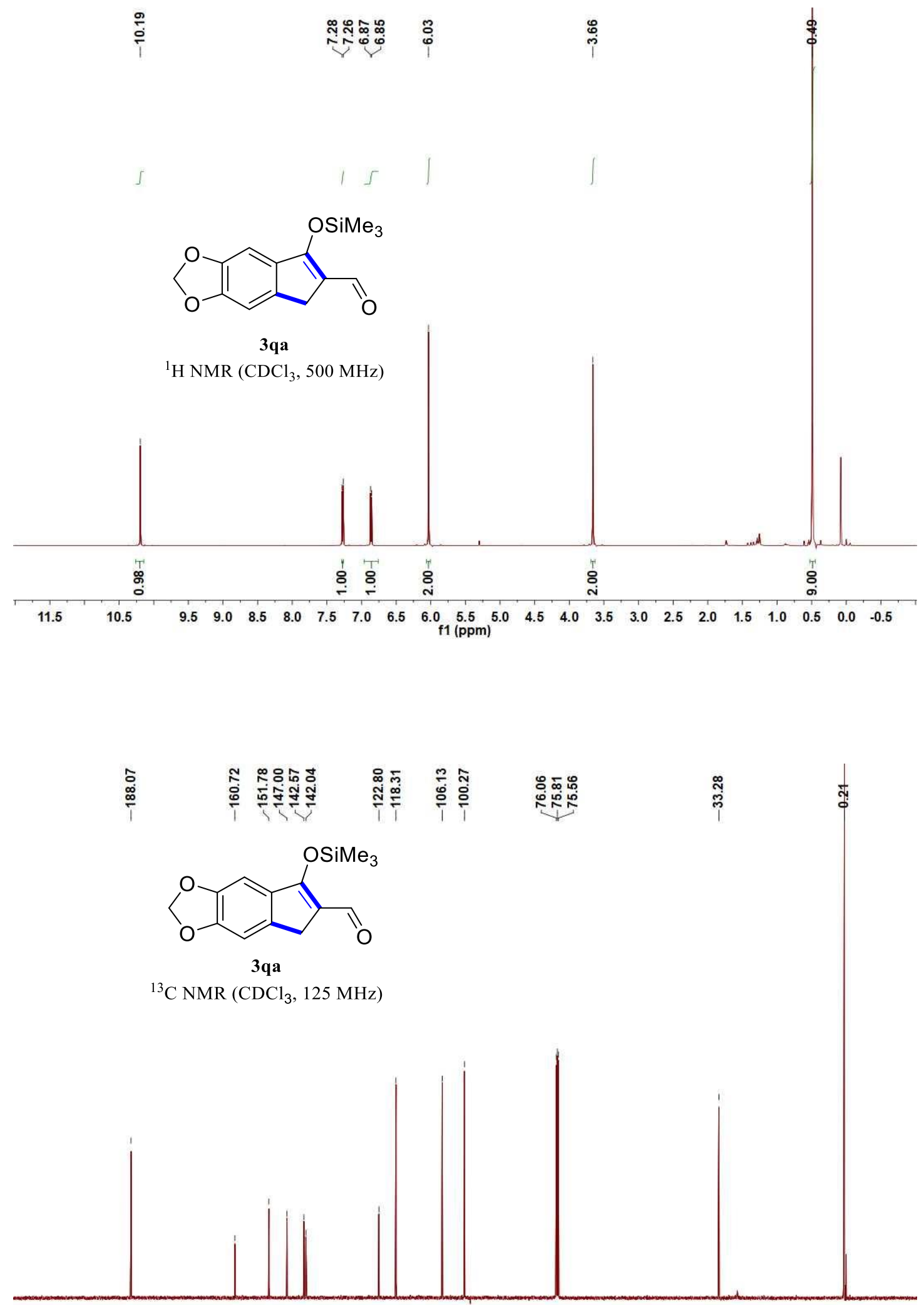

$\begin{array}{llllllllllllllllllllll}210 & 200 & 190 & 180 & 170 & 160 & 150 & 140 & 130 & 120 & 110 \begin{array}{l}100 \\ \mathrm{f} 1(\mathrm{ppm})\end{array} & 90 & 80 & 70 & 60 & 50 & 40 & 30 & 20 & 10 & 0 & -10\end{array}$ 

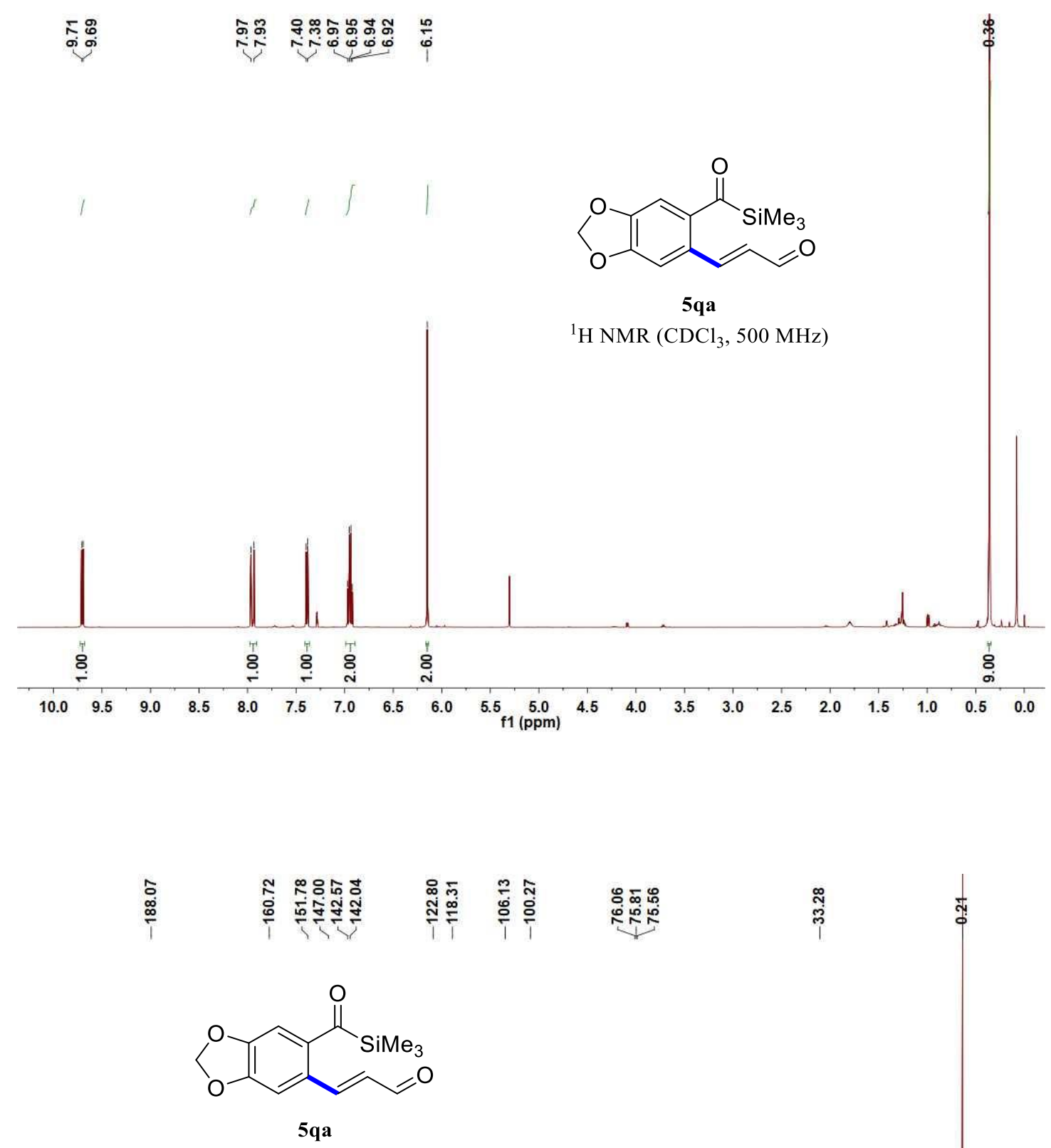

${ }^{13} \mathrm{C} \mathrm{NMR}\left(\mathrm{CDCl}_{3}, 125 \mathrm{MHz}\right)$

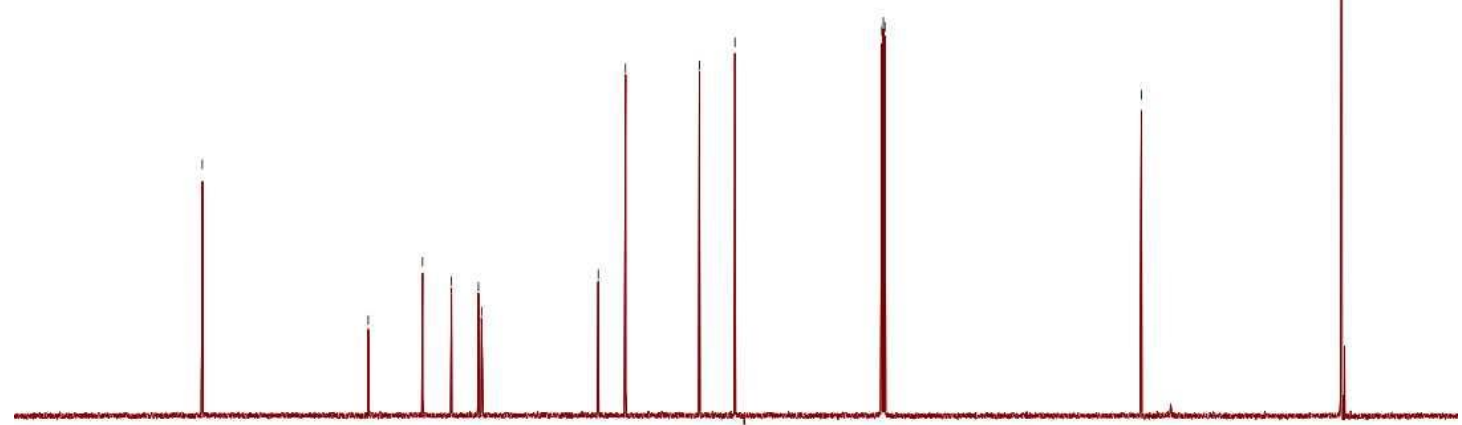

$\begin{array}{llllllllllllllllllllll}210 & 200 & 190 & 180 & 170 & 160 & 150 & 140 & 130 & 120 & 110 \begin{array}{l}100 \\ \mathrm{f} 1(\mathrm{ppm})\end{array} & 90 & 80 & 70 & 60 & 50 & 40 & 30 & 20 & 10 & 0 & -10\end{array}$ 


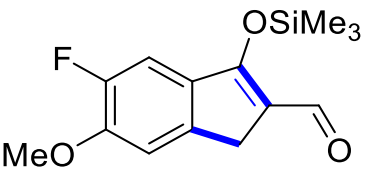

3ra

${ }^{1} \mathrm{H} \mathrm{NMR}\left(\mathrm{CDCl}_{3}, 500 \mathrm{MHz}\right)$

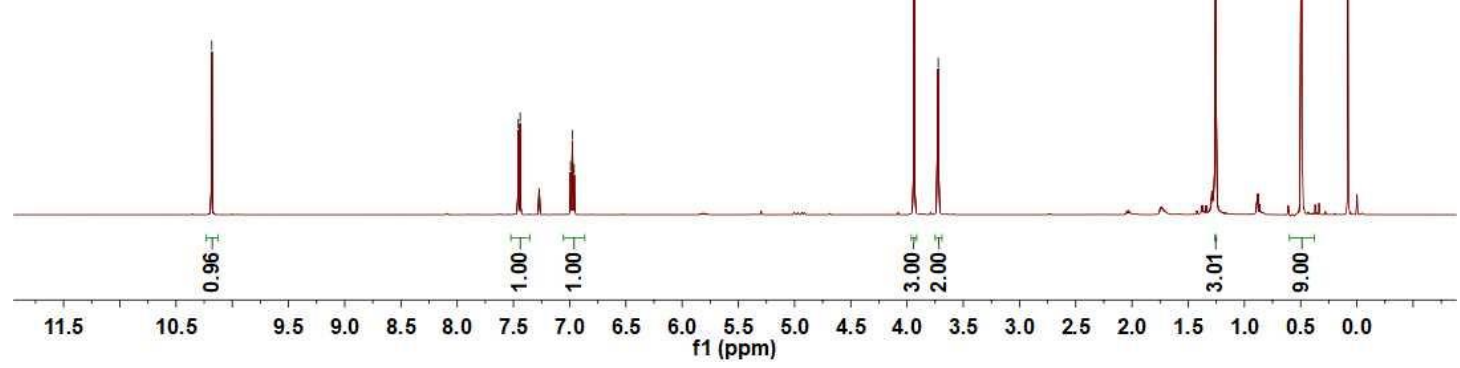

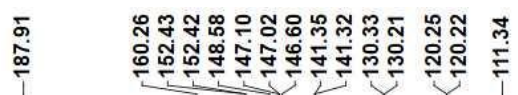
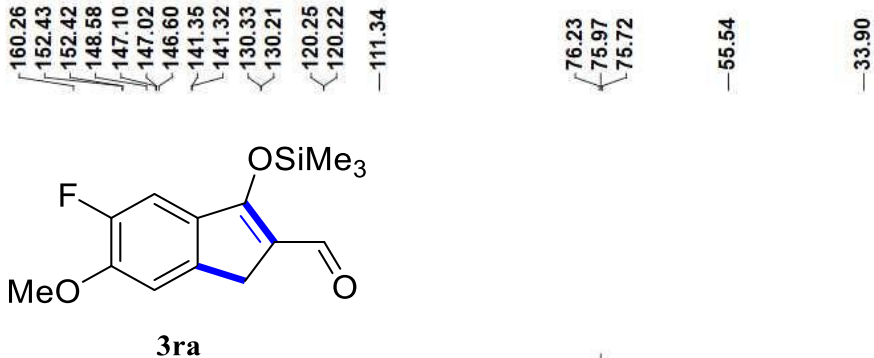

${ }^{13} \mathrm{C} \mathrm{NMR}\left(\mathrm{CDCl}_{3}, 125 \mathrm{MHz}\right)$

$\begin{array}{llllllllllll}! 10 & 200 & 190 & 180 & 170 & 160 & 150 & 140 & 130 & 120 & 110 & 100\end{array}$ 
$\stackrel{\circ}{\frac{0}{0}}$

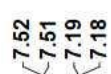

$\stackrel{0}{i}$

ঙ্ণ

S I
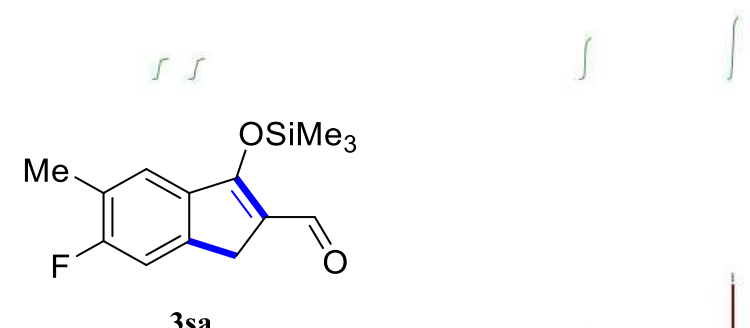

3 sa

${ }^{1} \mathrm{H}$ NMR $\left(\mathrm{CDCl}_{3}, 500 \mathrm{MHz}\right)$

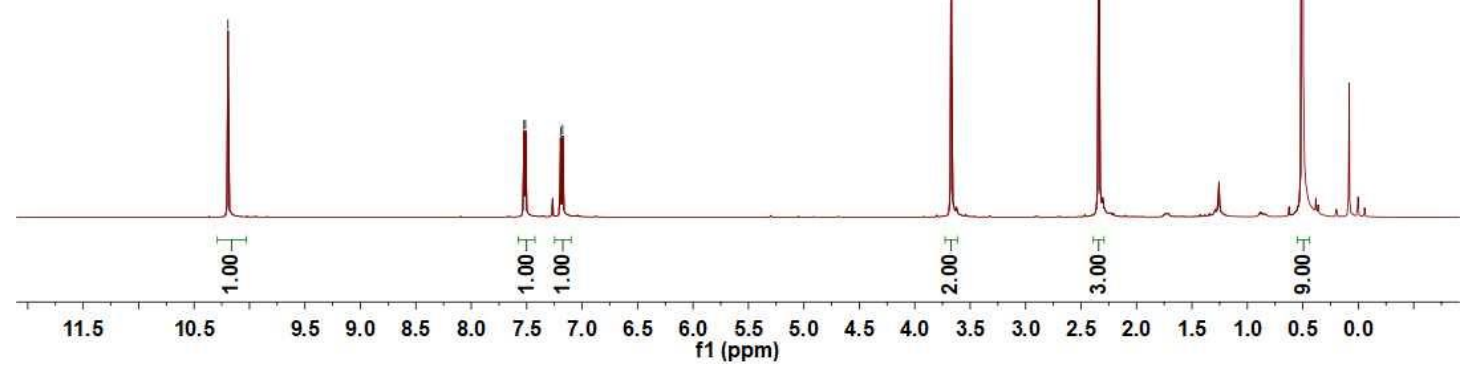

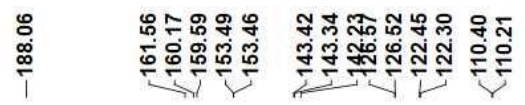

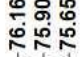

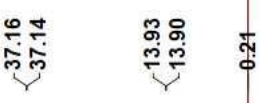

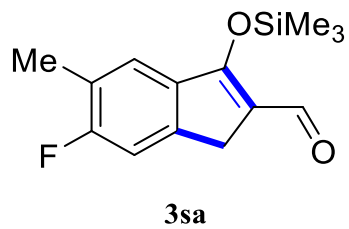

${ }^{13} \mathrm{C} \mathrm{NMR}\left(\mathrm{CDCl}_{3}, 125 \mathrm{MHz}\right)$

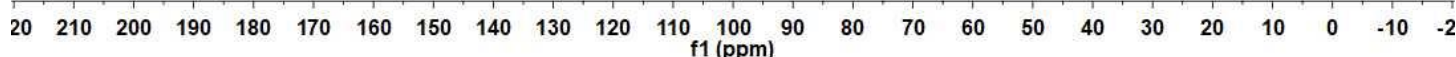




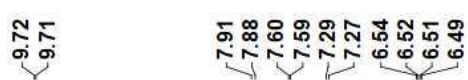

$\stackrel{\text { ָे }}{\text { i }}$<smiles>CCCCC(=O)c1cc(C)c(F)cc1/C=C/C=O</smiles>

5sa

${ }^{1} \mathrm{H} \mathrm{NMR}\left(\mathrm{CDCl}_{3}, 500 \mathrm{MHz}\right)$

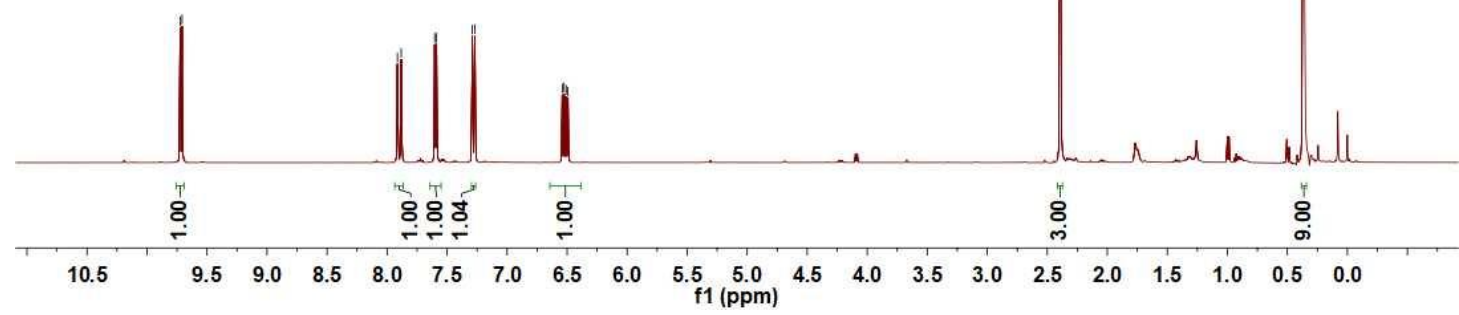

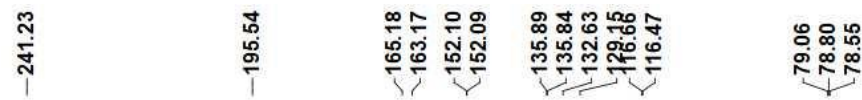

象它<smiles>CSC(=O)c1cc(C)c(F)cc1/C=C/C=O</smiles>

${ }^{13} \mathrm{C} \mathrm{NMR}\left(\mathrm{CDCl}_{3}, 125 \mathrm{MHz}\right)$

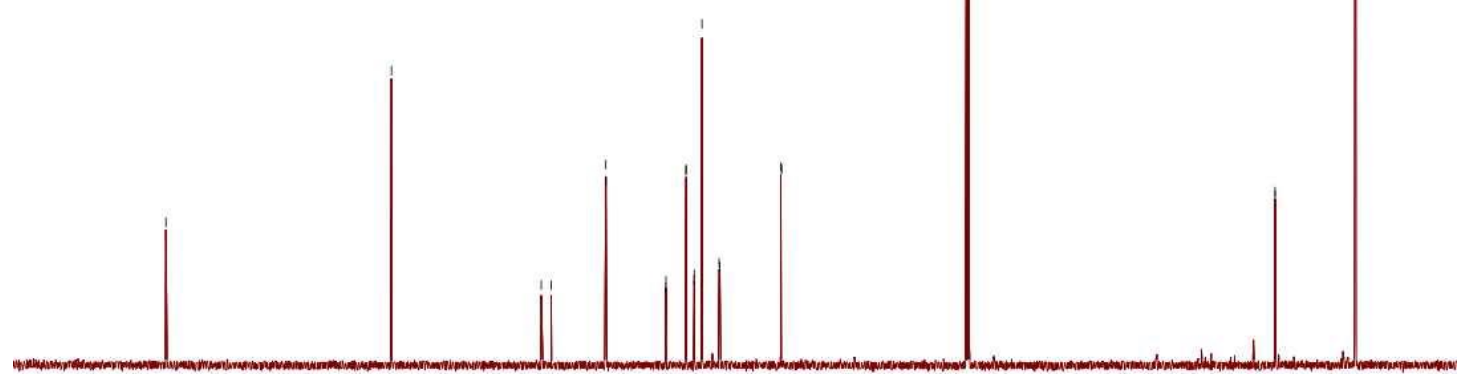

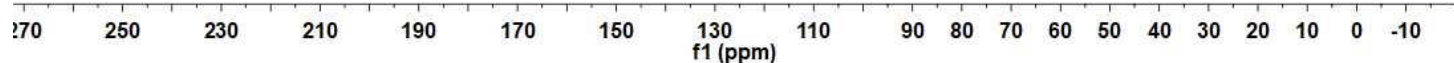




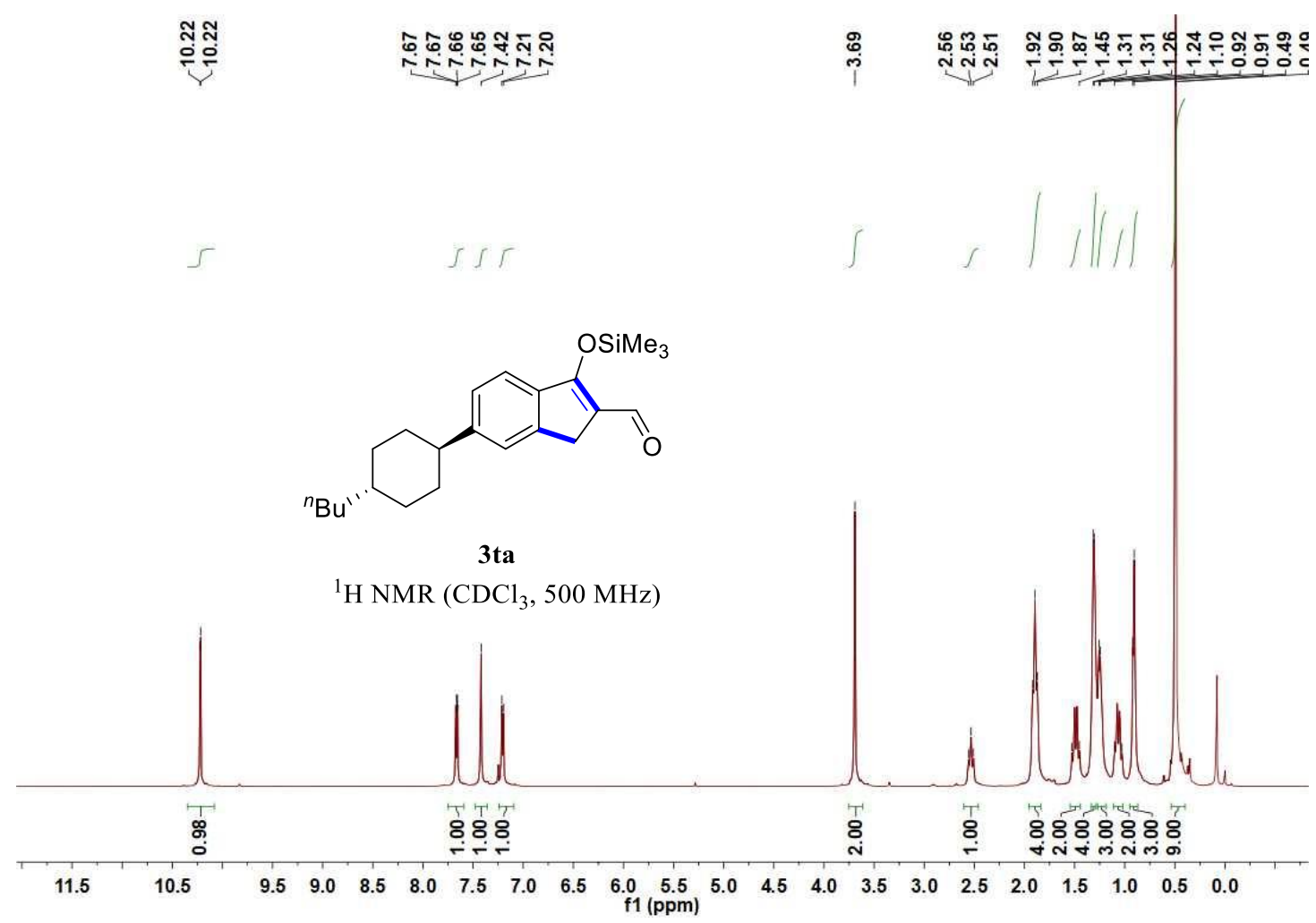

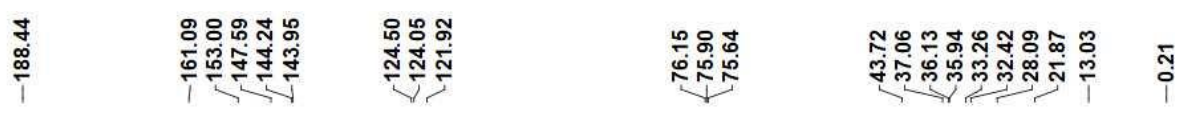

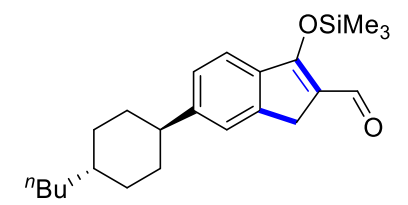

3ta

${ }^{13} \mathrm{C} \mathrm{NMR}\left(\mathrm{CDCl}_{3}, 125 \mathrm{MHz}\right)$

$\begin{array}{llllllllllllllllllllll}210 & 200 & 190 & 180 & 170 & 160 & 150 & 140 & 130 & 120 & 110 \underset{\mathrm{f} 1}{100}(\mathrm{ppm}) & 90 & 80 & 70 & 60 & 50 & 40 & 30 & 20 & 10 & 0 & -10\end{array}$ 


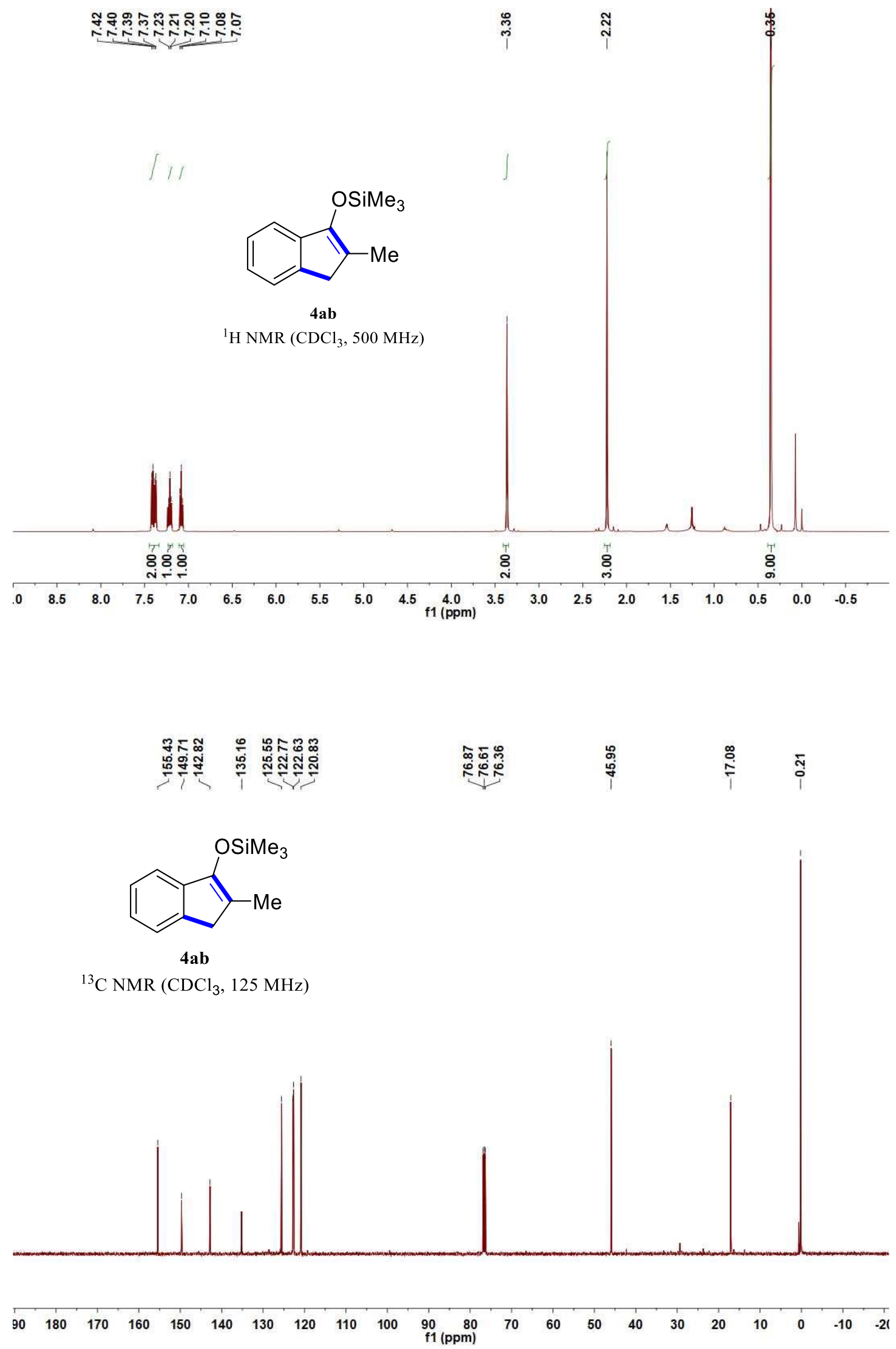




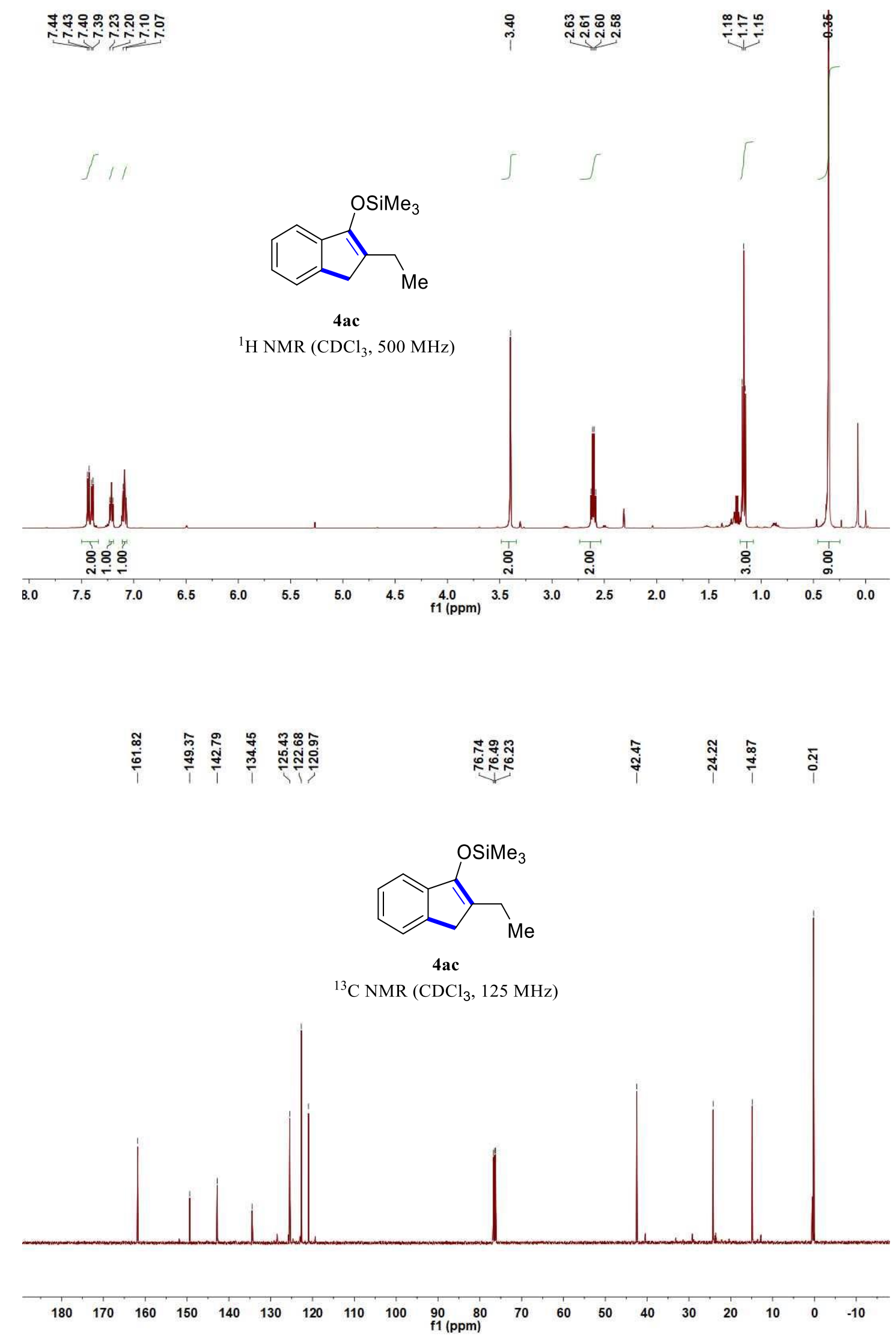




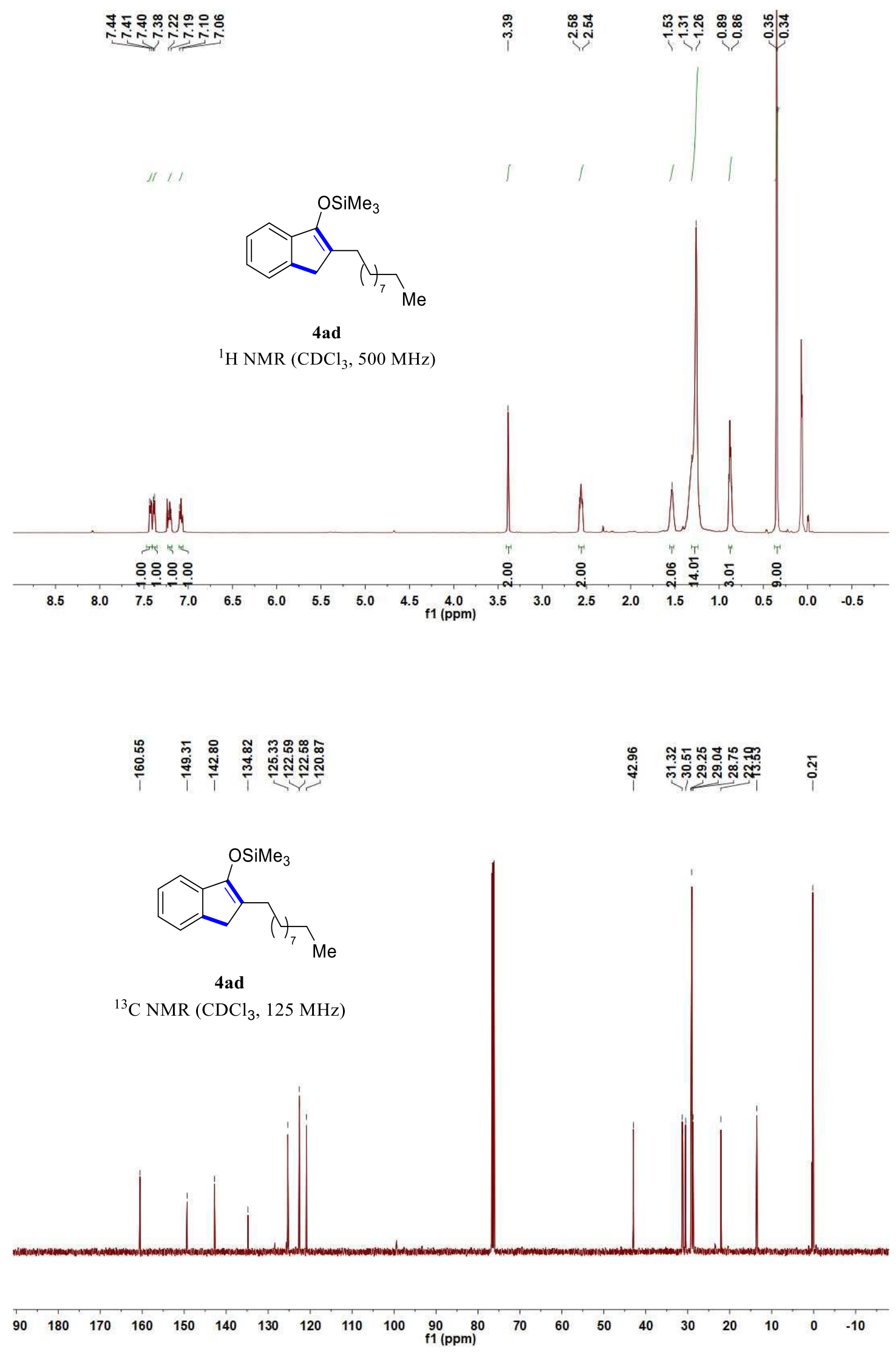



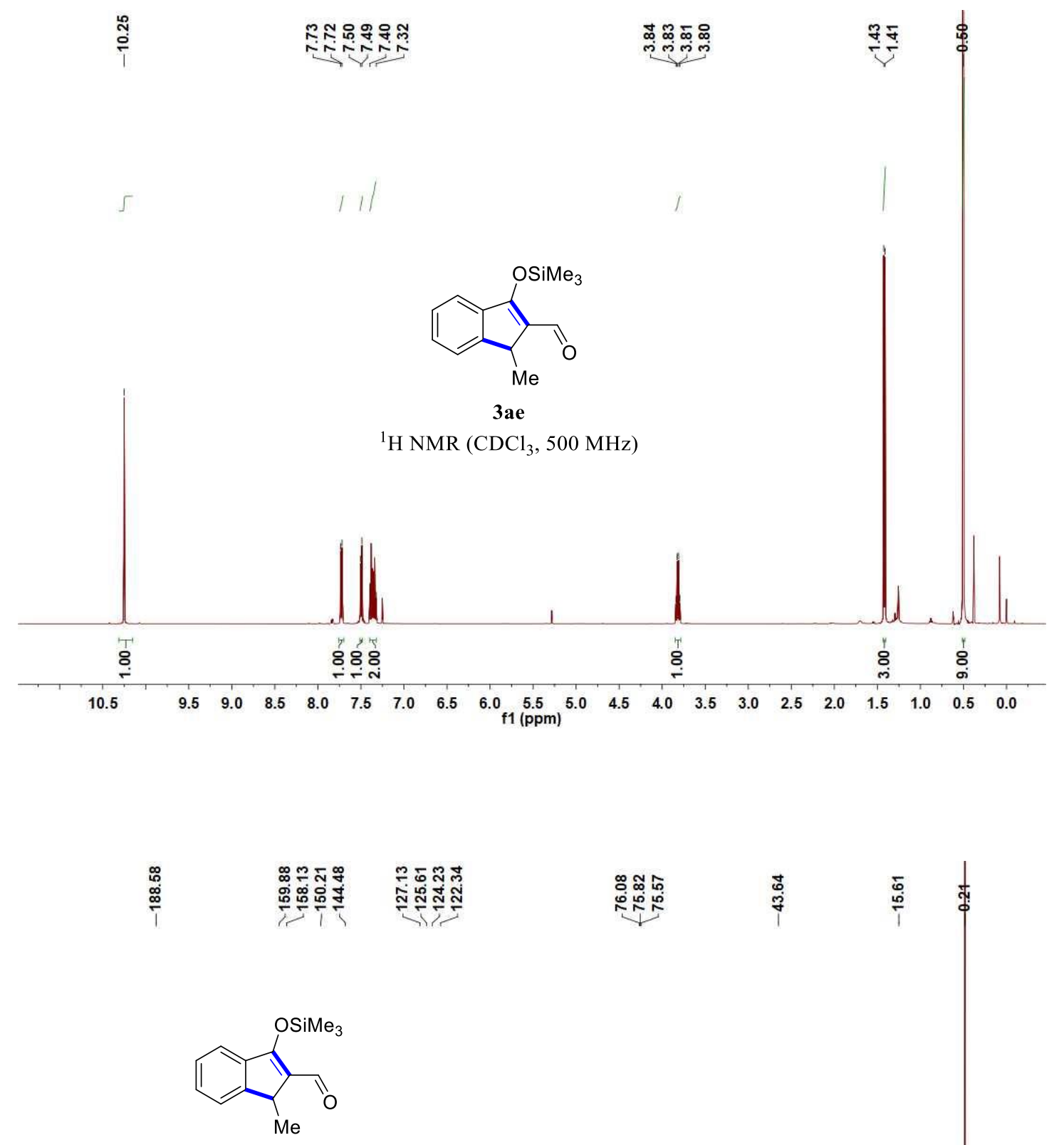

3 ae

${ }^{13} \mathrm{C} \mathrm{NMR}\left(\mathrm{CDCl}_{3}, 125 \mathrm{MHz}\right)$

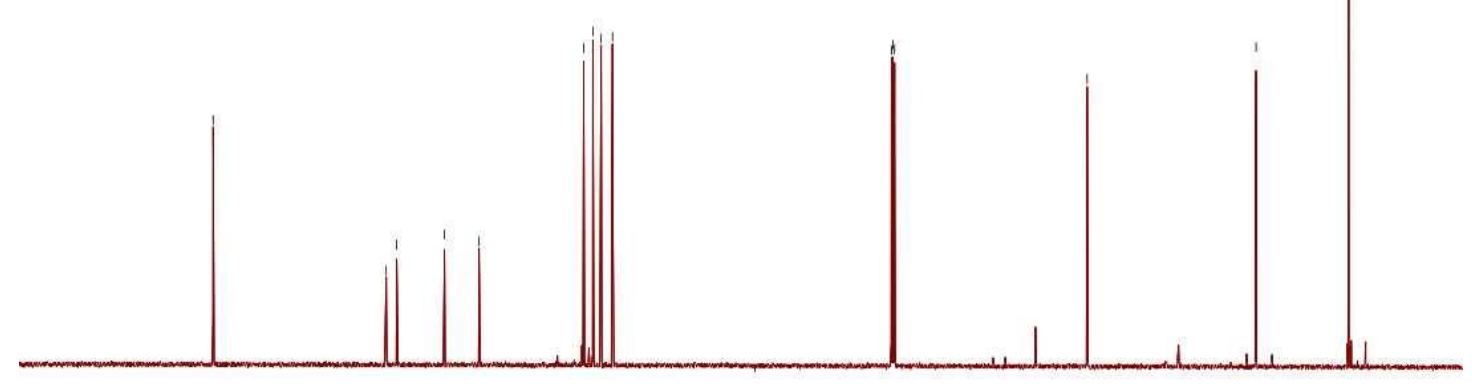

$\begin{array}{llllllllllllllllllllllllllll}20 & 210 & 200 & 190 & 180 & 170 & 160 & 150 & 140 & 130 & 120 & 110 & 100 & 90 & 80 & 70 & 60 & 50 & 40 & 30 & 20 & 10 & 0 & -10\end{array}$ 

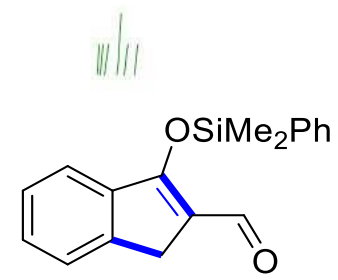

3ua

${ }^{1} \mathrm{H}$ NMR $\left(\mathrm{CDCl}_{3}, 500 \mathrm{MHz}\right)$
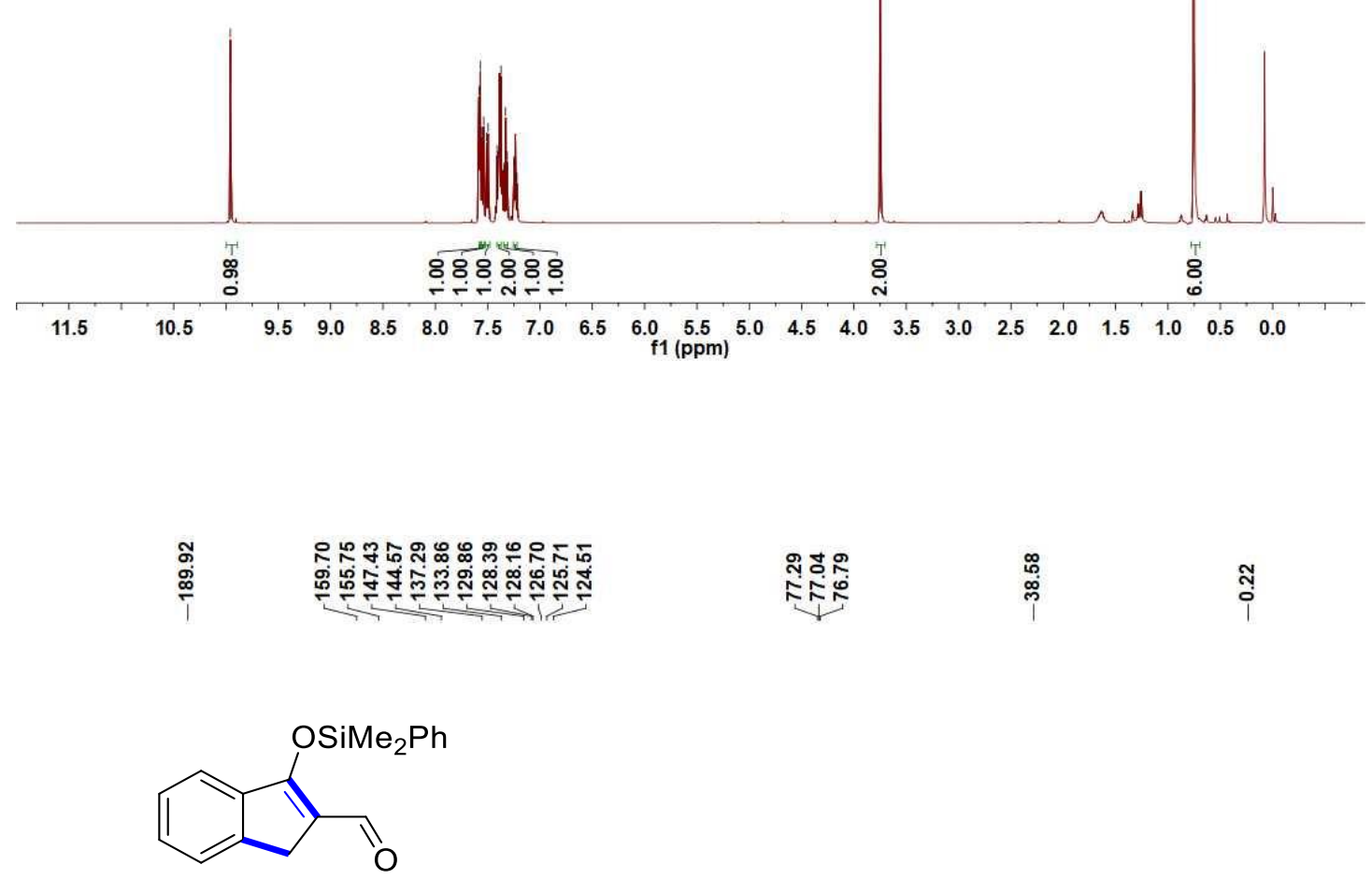

3 ua

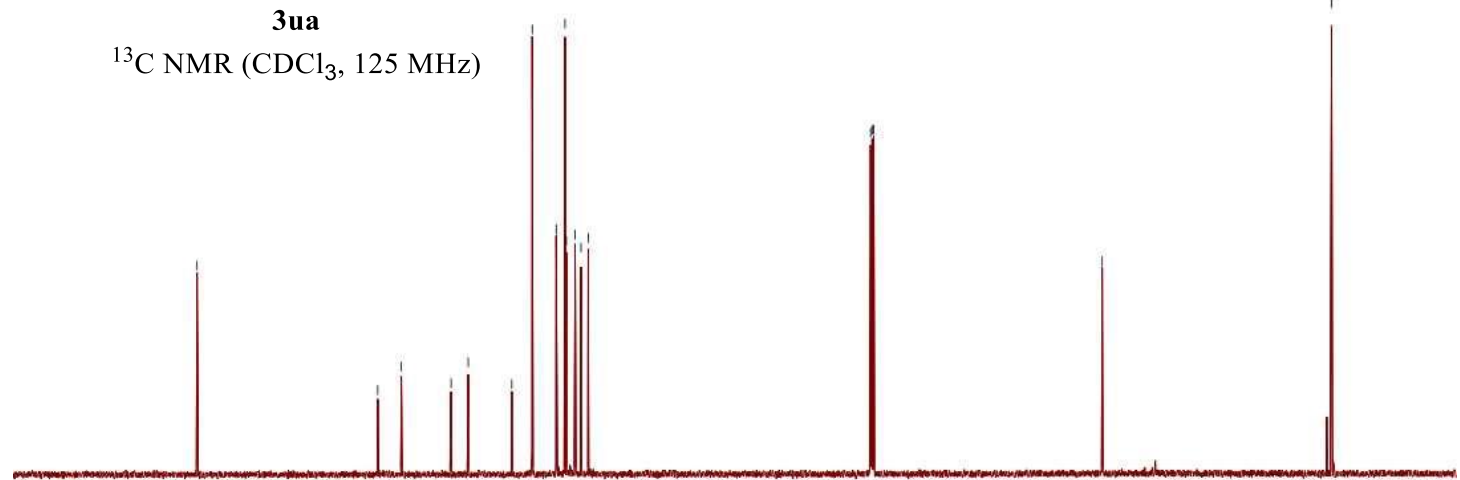

$\begin{array}{llllllllllllllllllllllllllllllll}20 & 210 & 200 & 190 & 180 & 170 & 160 & 150 & 140 & 130 & 120 & 110 & 100 & 90 & 80 & 70 & 60 & 50 & 40 & 30 & 20 & 10 & 0 & -10 & -2\end{array}$ 


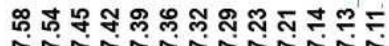

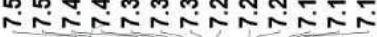
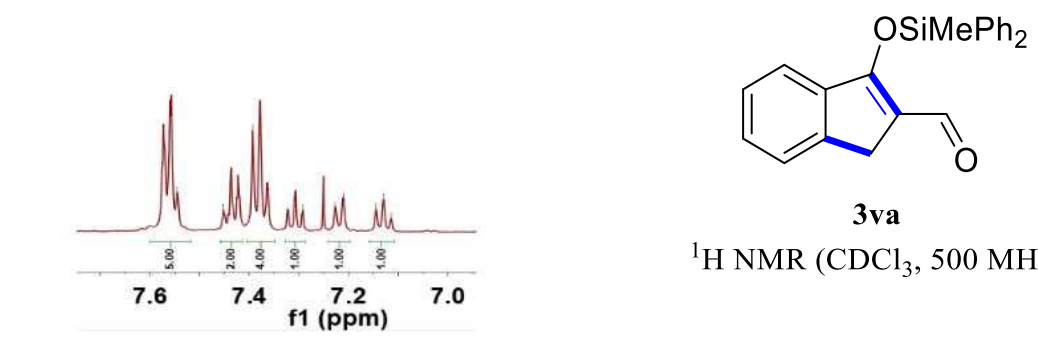

3va

${ }^{1} \mathrm{H}$ NMR $\left(\mathrm{CDCl}_{3}, 500 \mathrm{MHz}\right)$
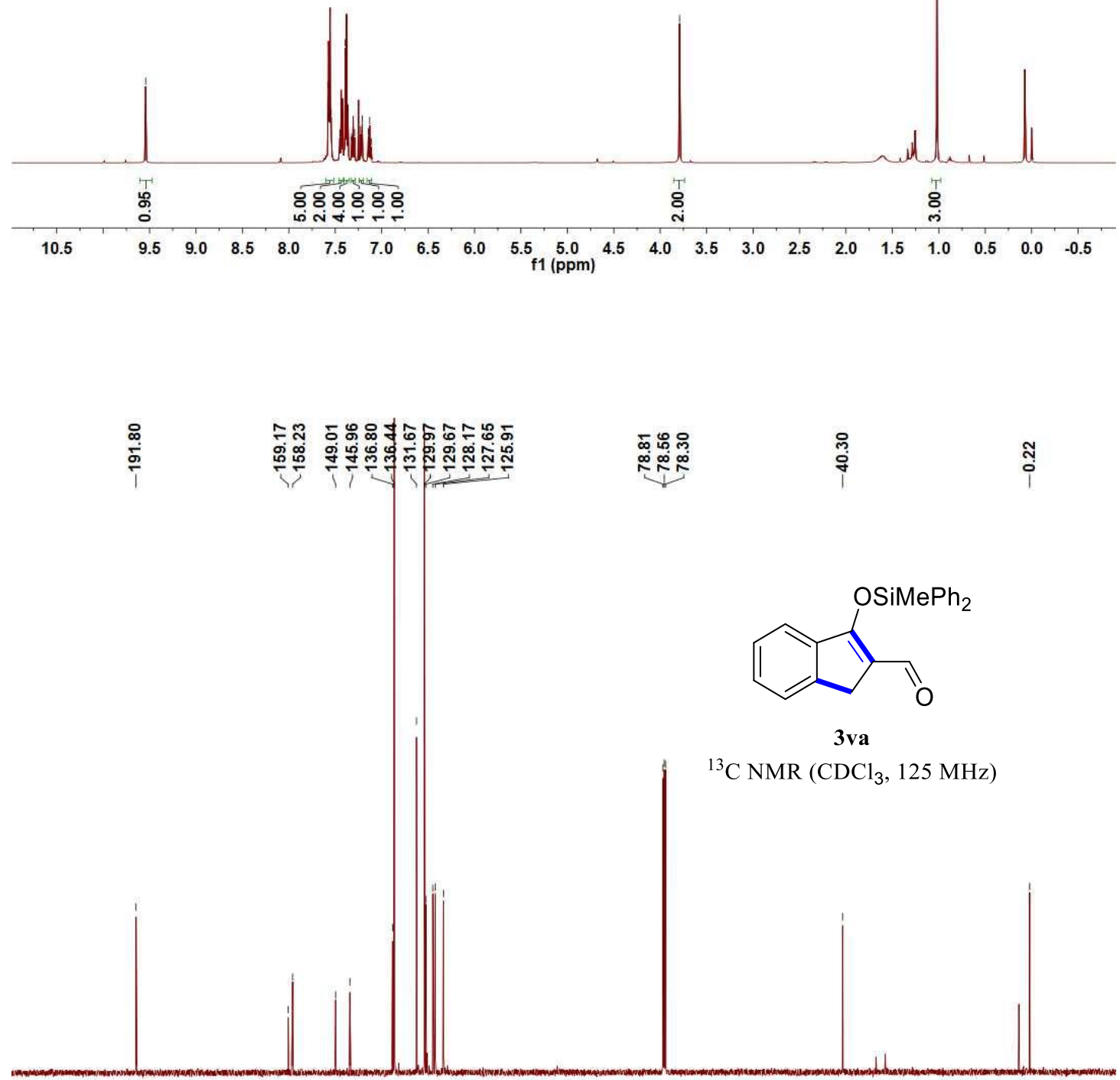

$\begin{array}{lllllllllllllllllllllll}210 & 200 & 190 & 180 & 170 & 160 & 150 & 140 & 130 & 120 & 110 & \begin{array}{l}100 \\ \mathrm{f} 1(\mathrm{ppm})\end{array} & 90 & 80 & 70 & 60 & 50 & 40 & 30 & 20 & 10 & 0 & -10\end{array}$ 
$\stackrel{\infty}{\infty}$

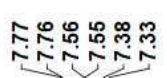

$\stackrel{\stackrel{\leftrightarrow}{i}}{i}$
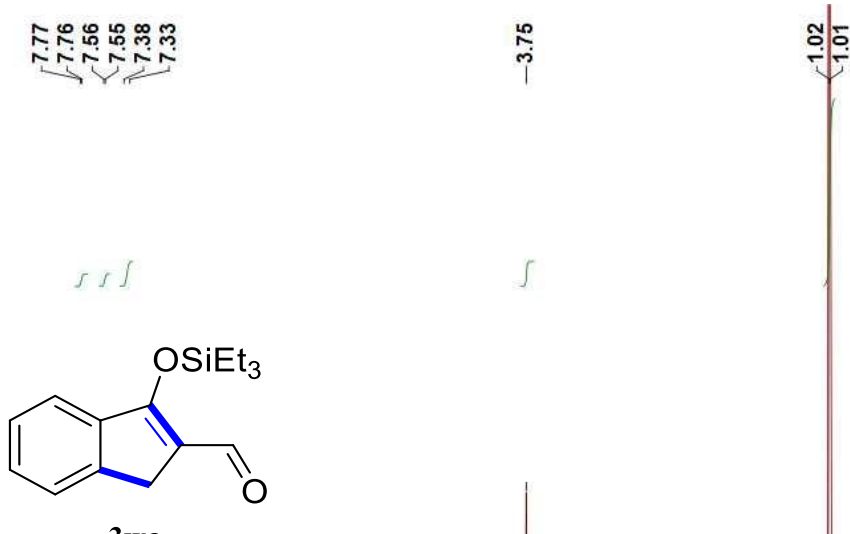

${ }^{1} \mathrm{H} \mathrm{NMR}\left(\mathrm{CDCl}_{3}, 500 \mathrm{MHz}\right)$
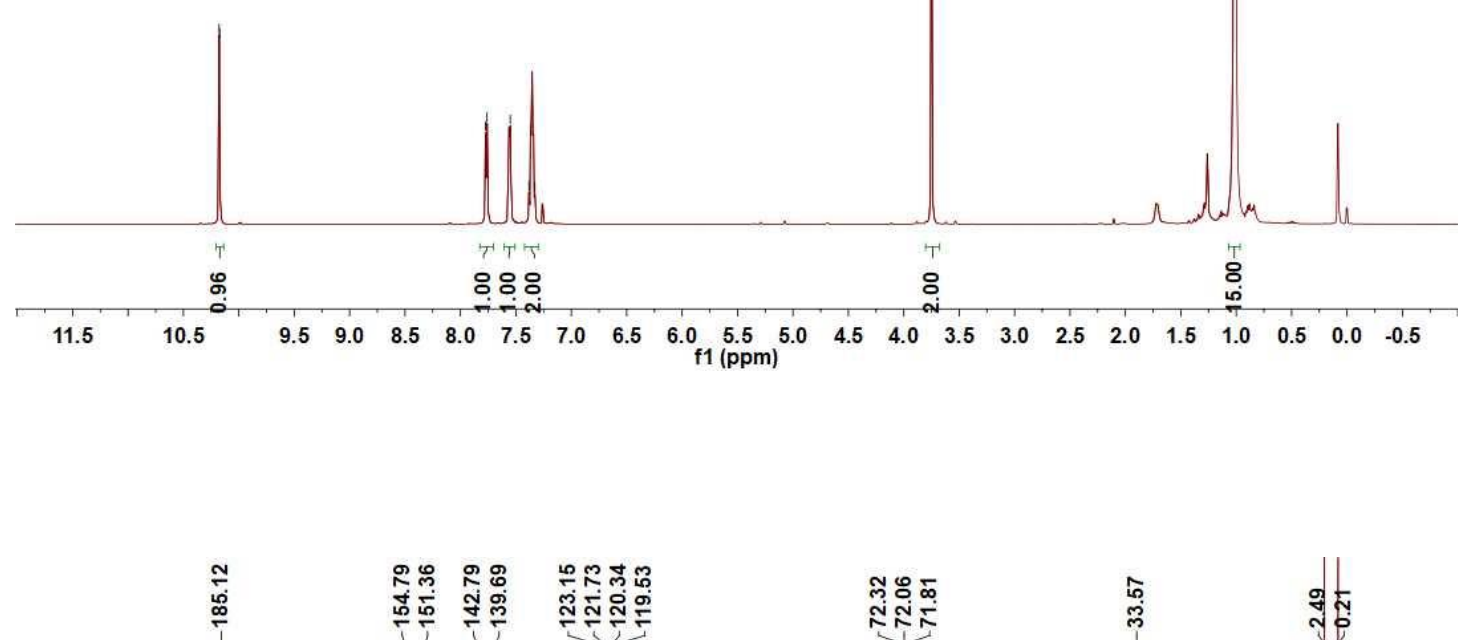

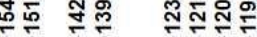

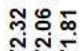

î.
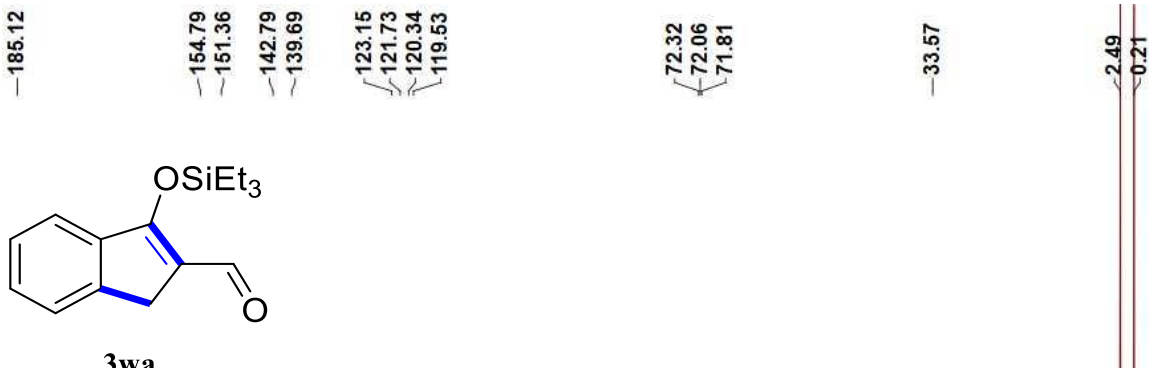

${ }^{13} \mathrm{C} \mathrm{NMR}\left(\mathrm{CDCl}_{3}, 125 \mathrm{MHz}\right)$

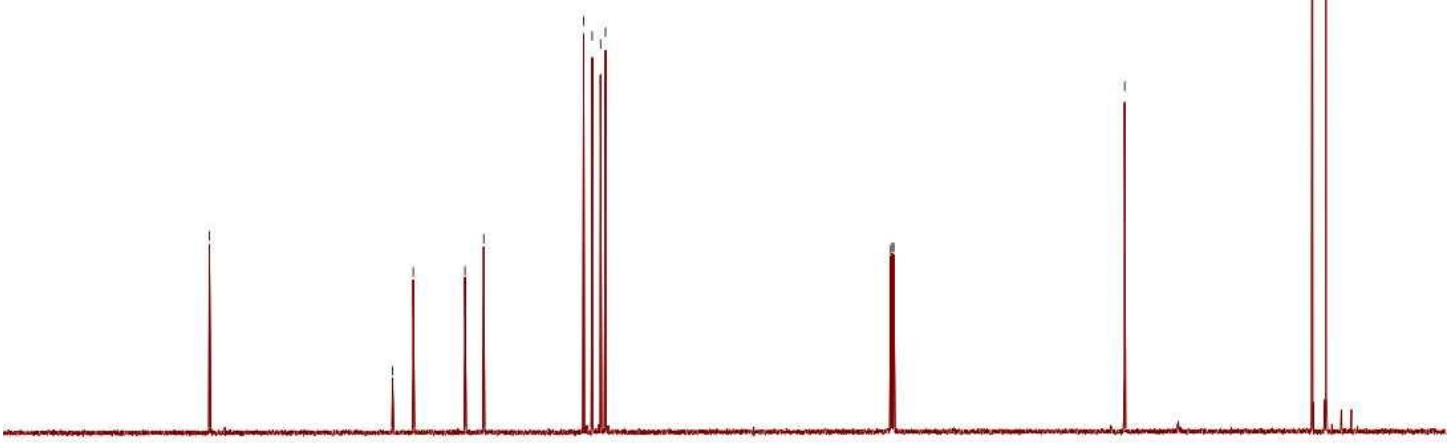

$\begin{array}{llllllllllllllllllllll}210 & 200 & 190 & 180 & 170 & 160 & 150 & 140 & 130 & 120 & 110 \begin{array}{c}100 \\ \mathrm{f} 1(\mathrm{ppm})\end{array} & 90 & 80 & 70 & 60 & 50 & 40 & 30 & 20 & 10 & 0 & -10\end{array}$ 


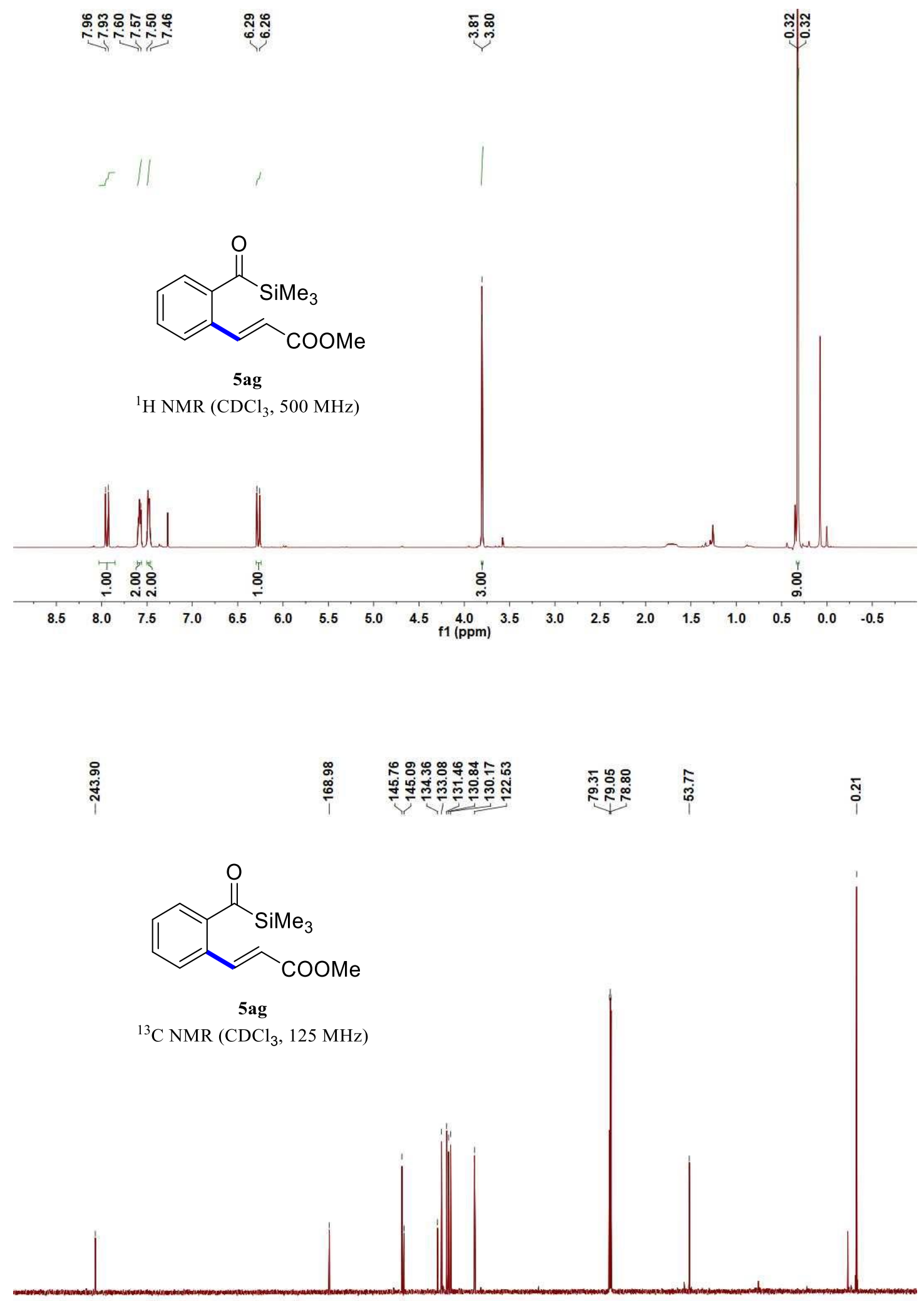

$\begin{array}{lllllllllllllllllllllllllll}10 & 250 & 230 & 210 & 190 & 170 & 150 & 130 & 110 & 90 & 80 & 70 & 60 & 50 & 40 & 30 & 20 & 10 & 0 & & \end{array}$ 

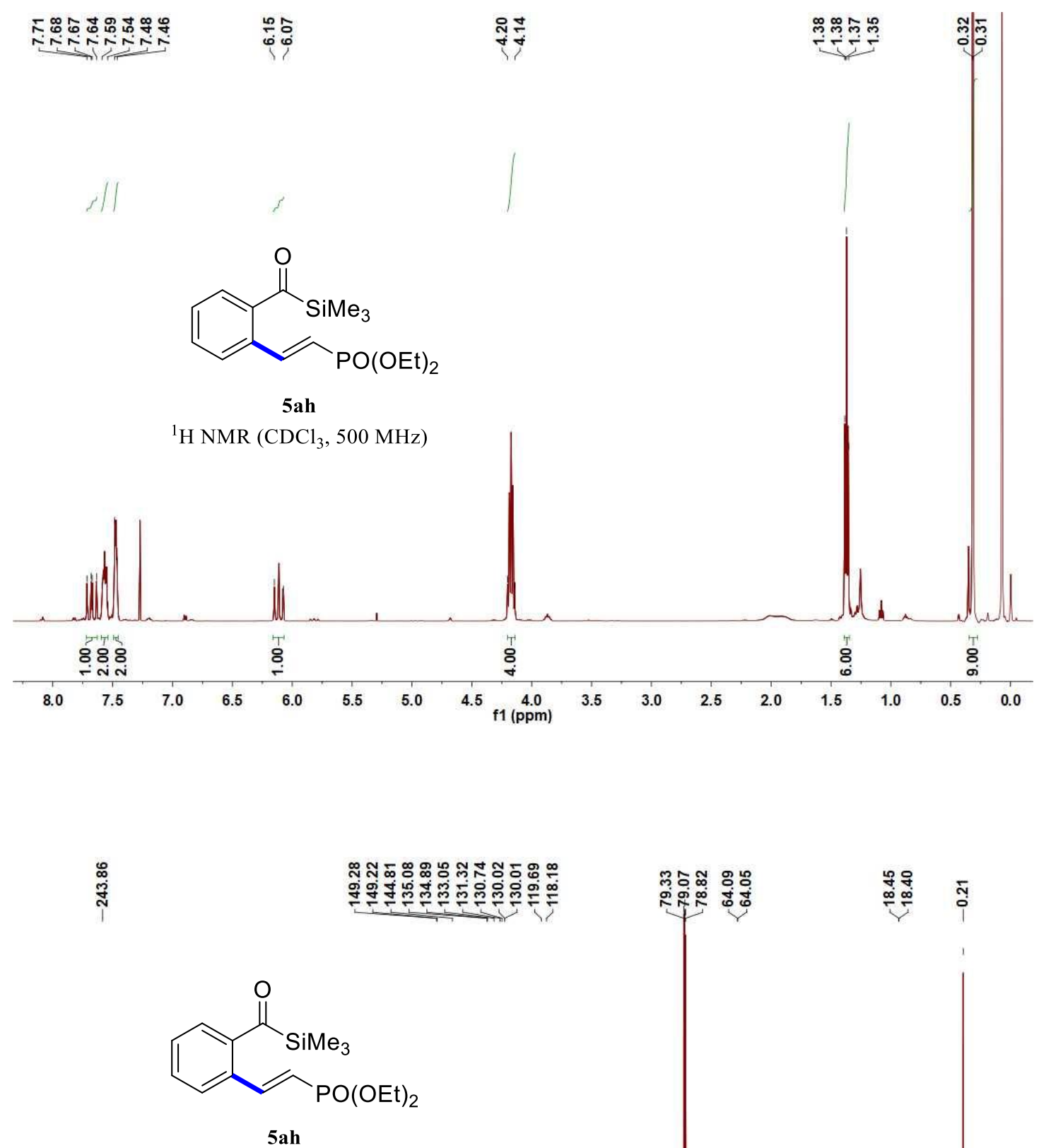

${ }^{13} \mathrm{C} \mathrm{NMR}\left(\mathrm{CDCl}_{3}, 125 \mathrm{MHz}\right)$

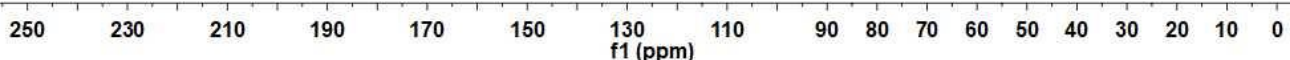




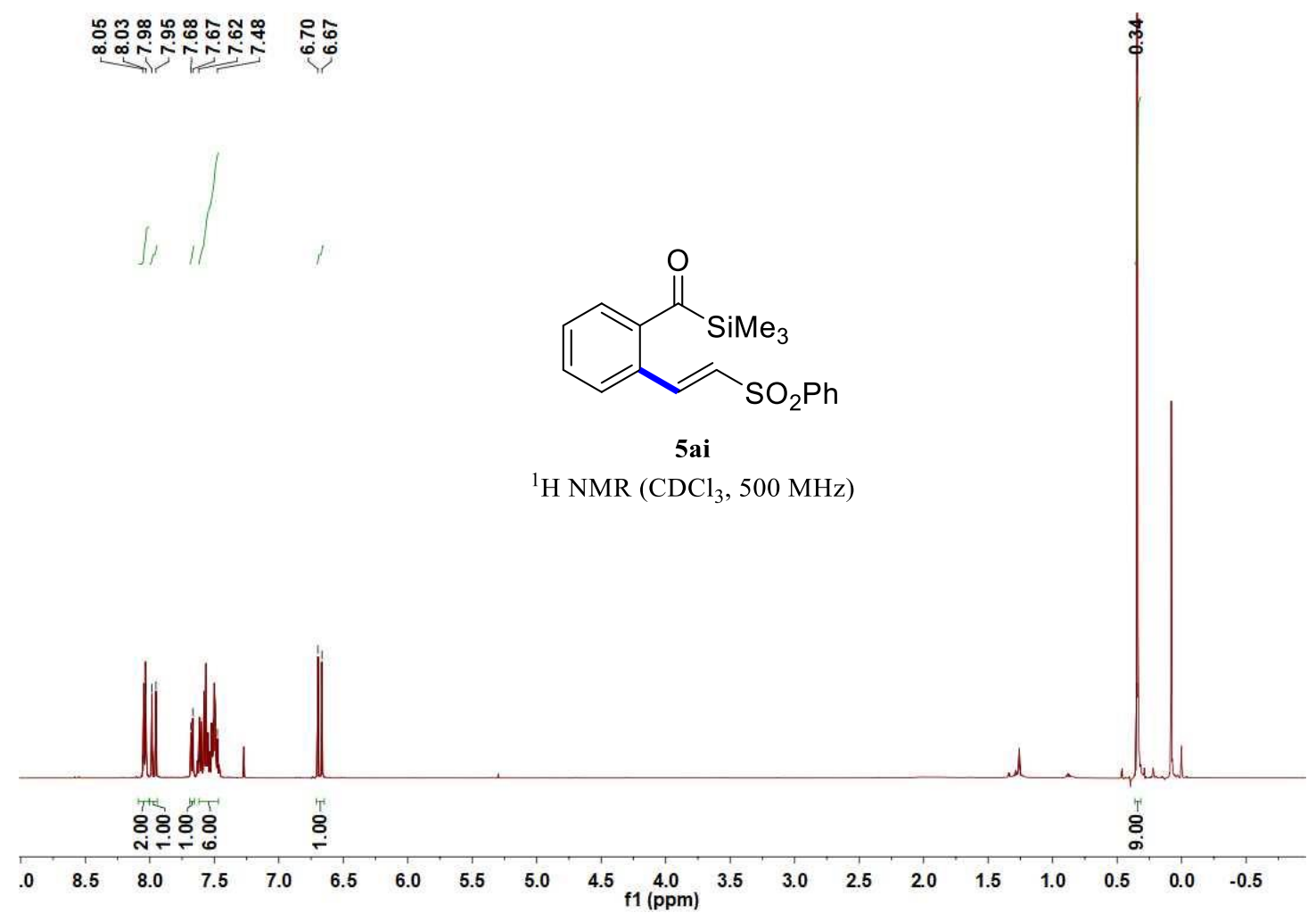

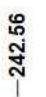<smiles></smiles>

5 ai

${ }^{13} \mathrm{C} \mathrm{NMR}\left(\mathrm{CDCl}_{3}, 125 \mathrm{MHz}\right)$

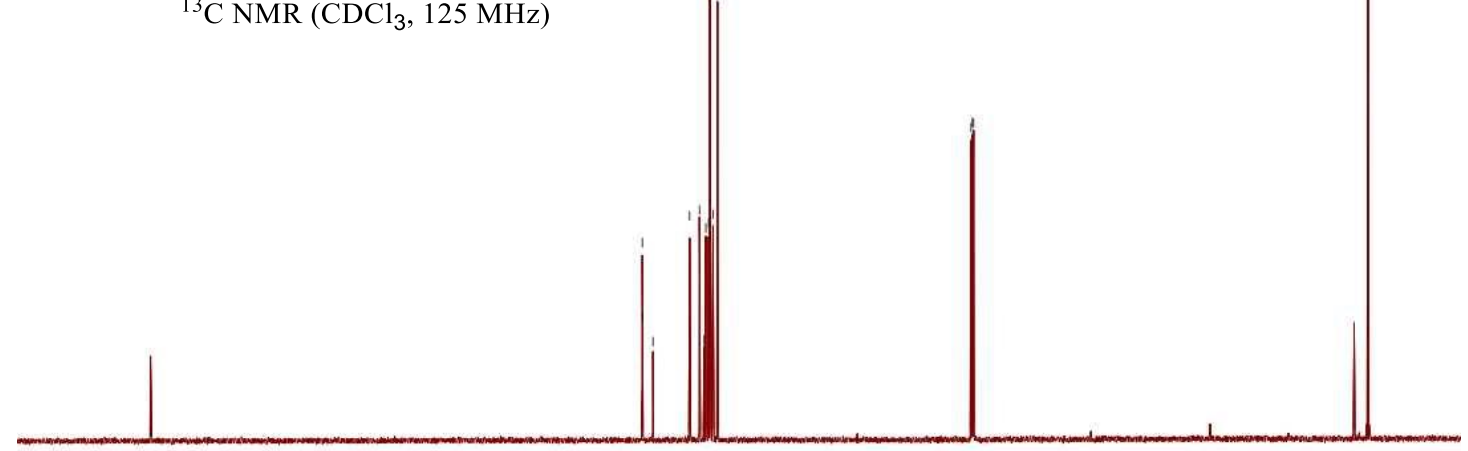

25
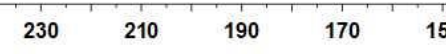

150 130
$\mathrm{f} 1(\mathrm{ppm})$ $\begin{array}{llllllllll}90 & 80 & 70 & 60 & 50 & 40 & 30 & 20 & 10 & 0\end{array}$ 


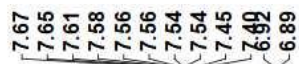
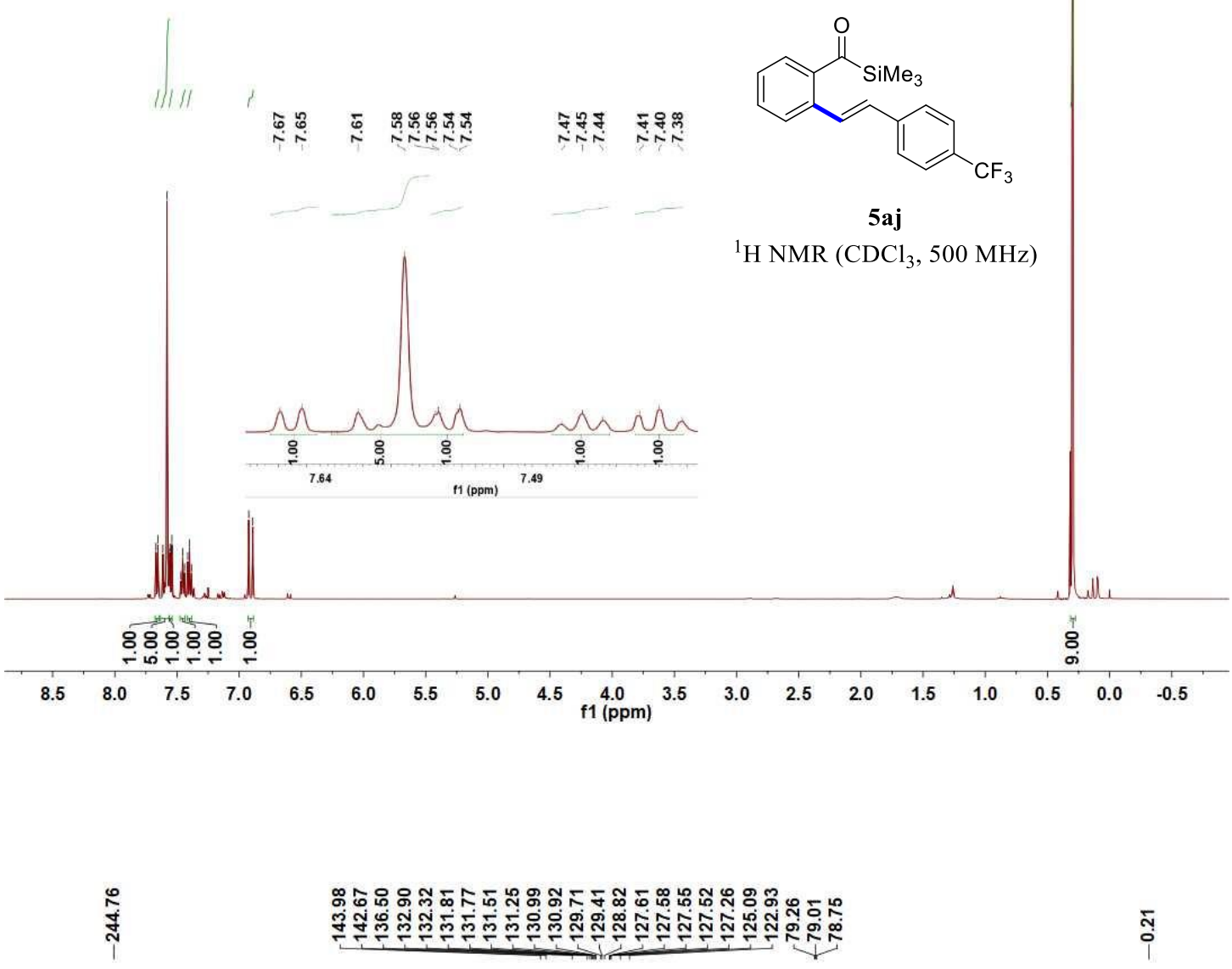

స్ํํ
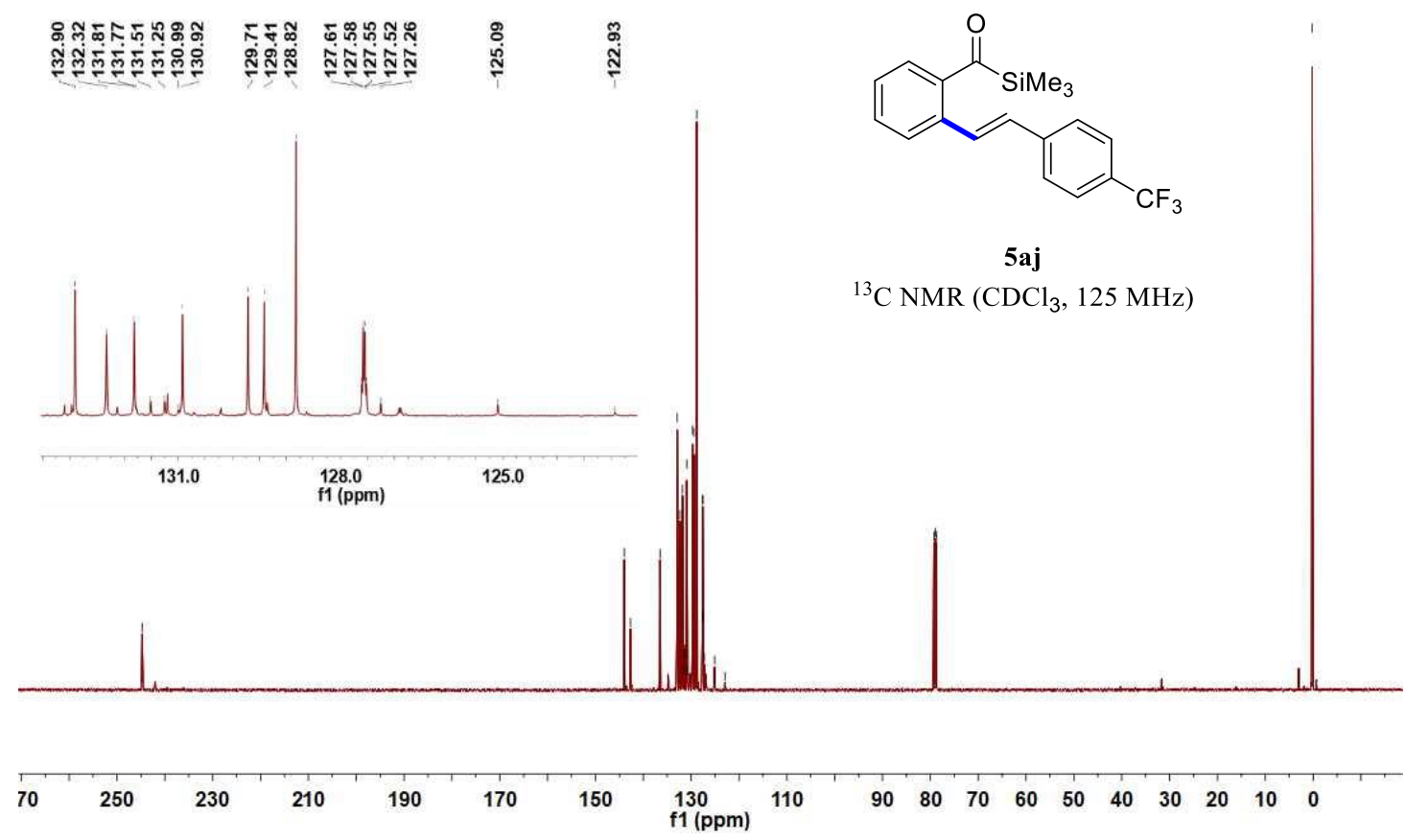

SI-104 

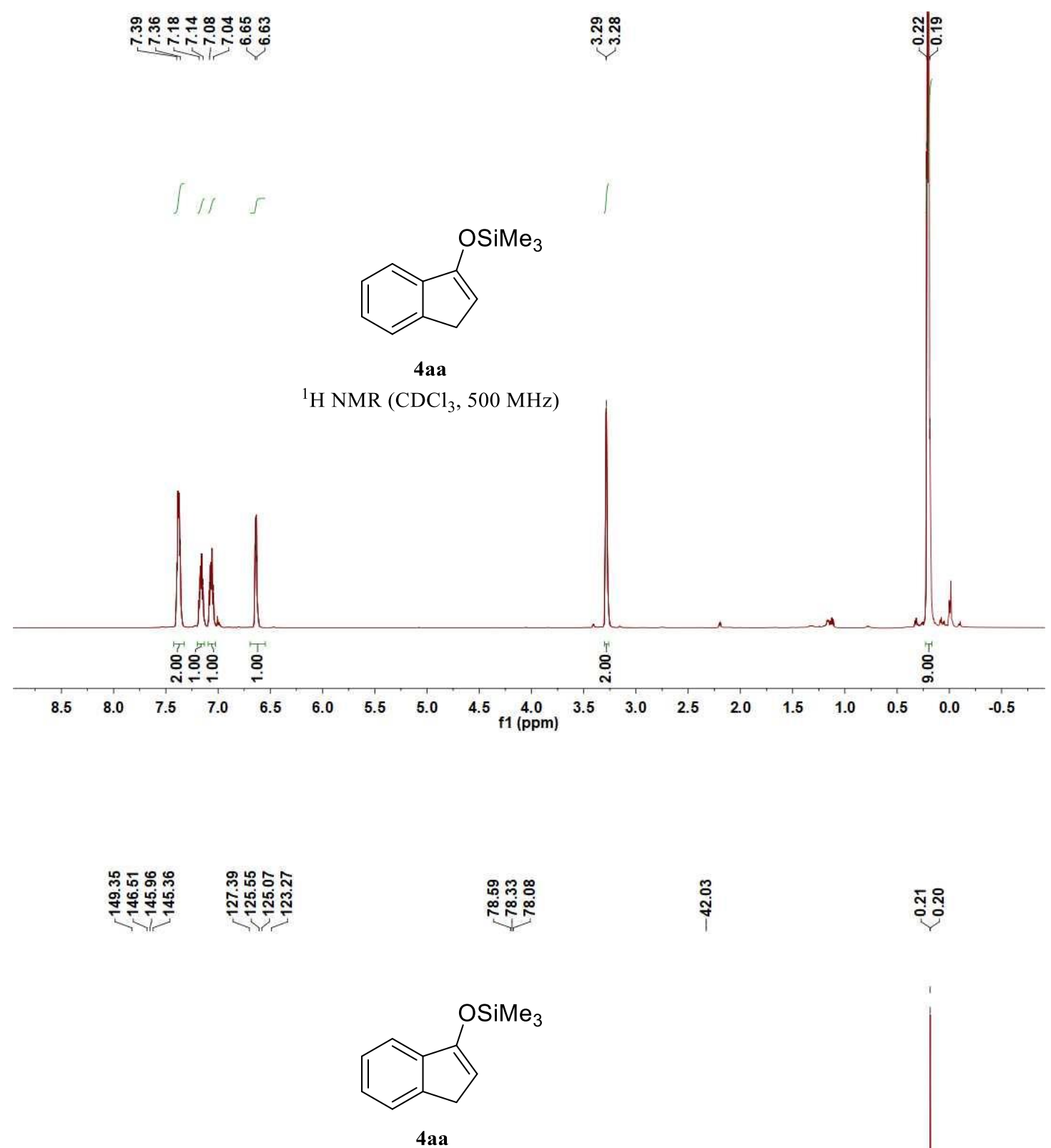

${ }^{13} \mathrm{C} \mathrm{NMR}\left(\mathrm{CDCl}_{3}, 125 \mathrm{MHz}\right)$

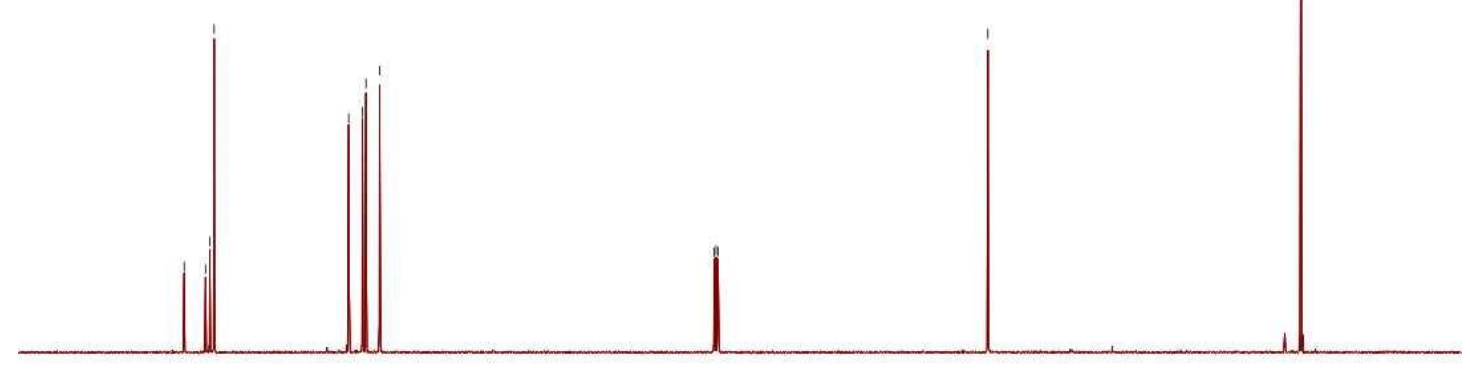

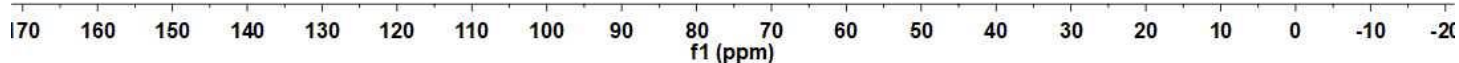




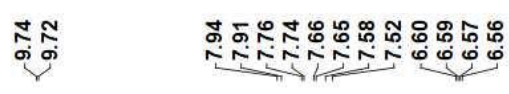
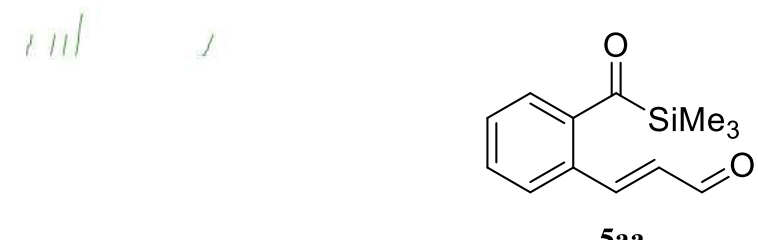

${ }^{1} \mathrm{H} \mathrm{NMR}\left(\mathrm{CDCl}_{3}, 500 \mathrm{MHz}\right)$
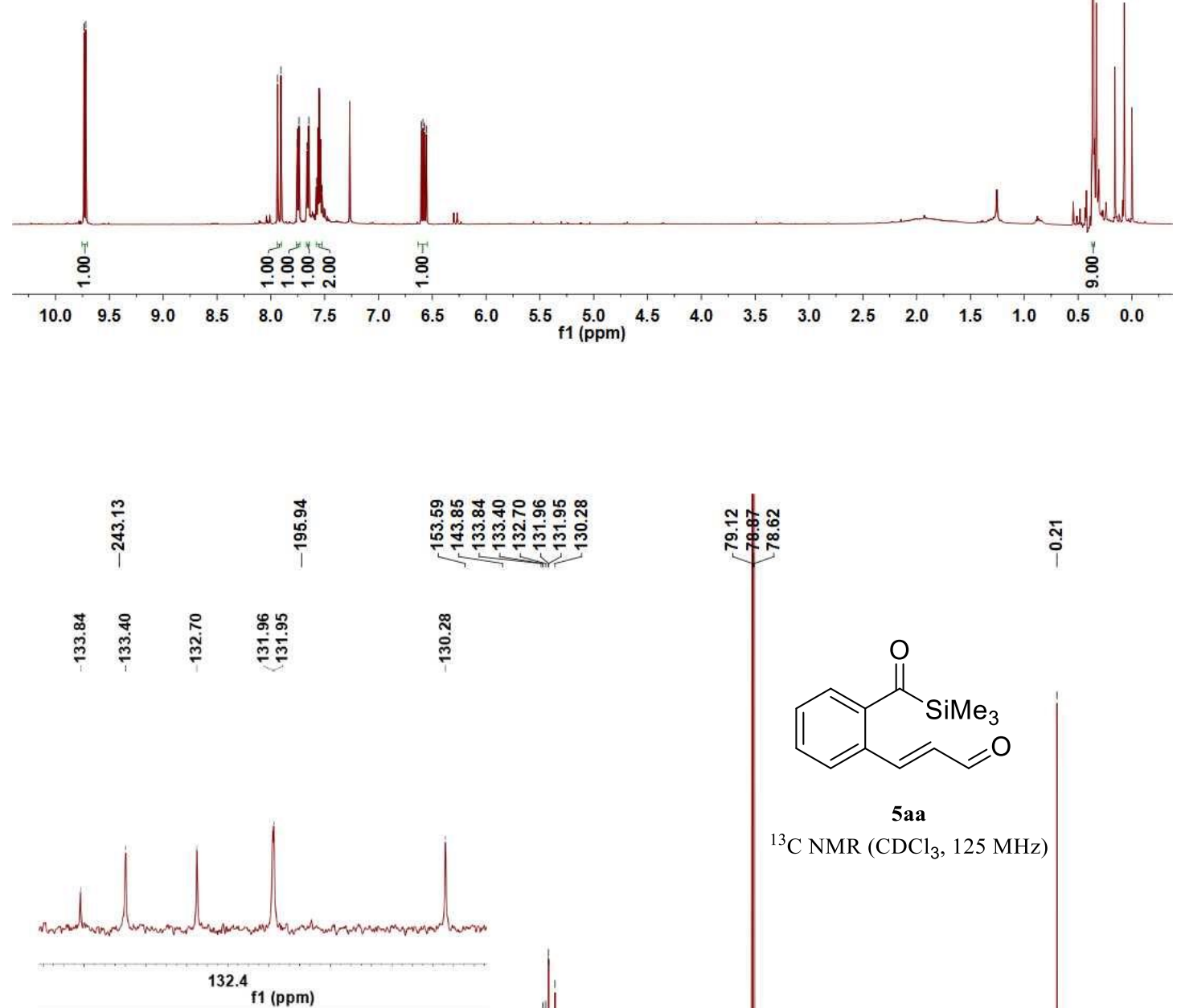

${ }^{13} \mathrm{C}$ NMR $\left(\mathrm{CDCl}_{3}, 125 \mathrm{MHz}\right)$

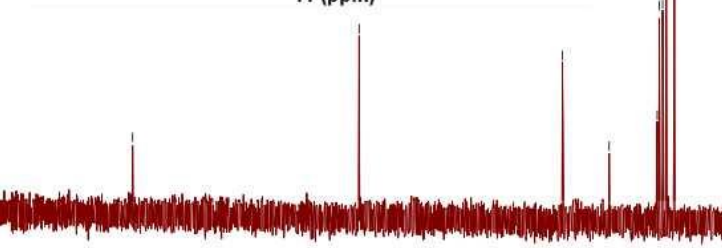

(1)

$\begin{array}{lllllllllllllllllll}70 & 250 & 230 & 210 & 190 & 170 & 150 & \underset{\mathrm{f} 1(\mathrm{ppm})}{130} & 90 & 80 & 70 & 60 & 50 & 40 & 30 & 20 & 10 & 0 & -10\end{array}$ 

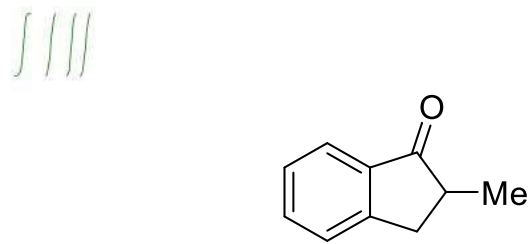

B

${ }^{1} \mathrm{H} \mathrm{NMR}\left(\mathrm{CDCl}_{3}, 500 \mathrm{MHz}\right)$

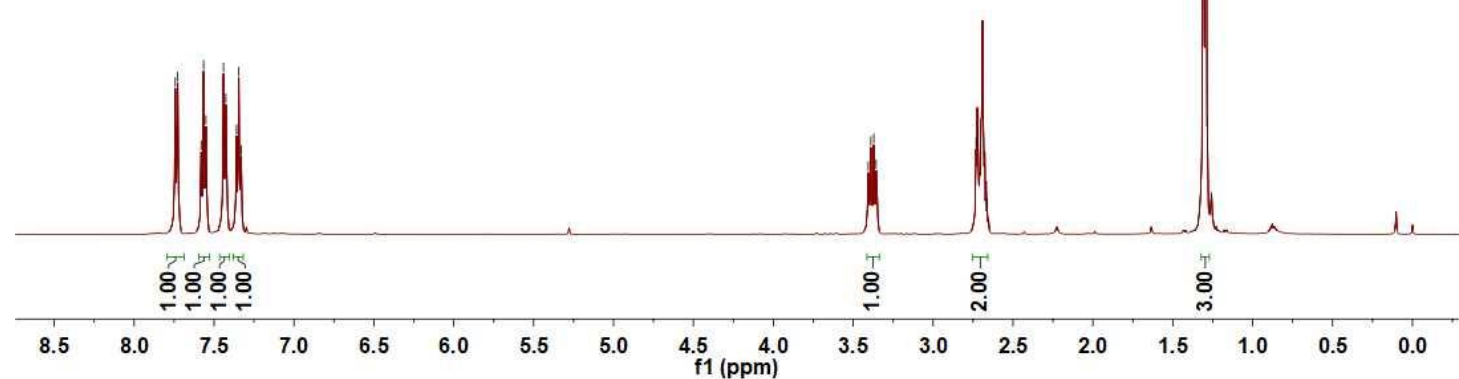

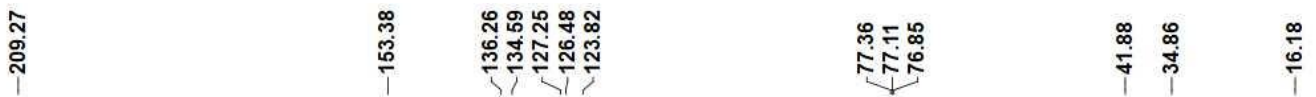<smiles>CC1Cc2ccccc2C1=O</smiles>

B

${ }^{13} \mathrm{C} \mathrm{NMR}\left(\mathrm{CDCl}_{3}, 125 \mathrm{MHz}\right)$

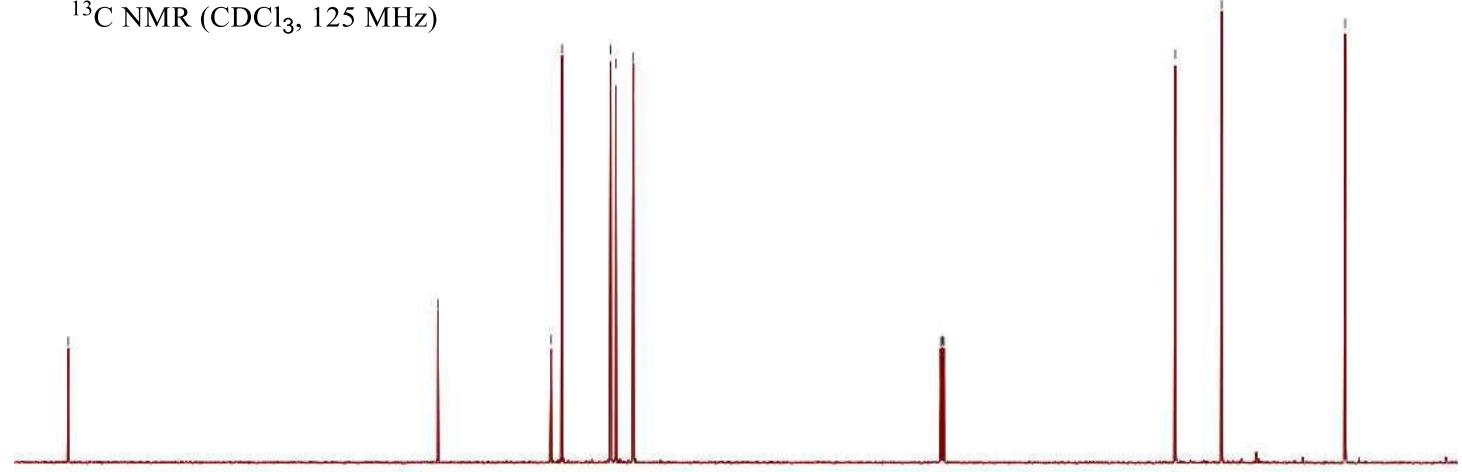

$\begin{array}{llllllllllllllllllllll}210 & 200 & 190 & 180 & 170 & 160 & 150 & 140 & 130 & 120 & \begin{array}{l}110 \\ 110\end{array} & 100 & 90 & 80 & 70 & 60 & 50 & 40 & 30 & 20 & 10 & 0\end{array}$ 


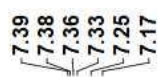

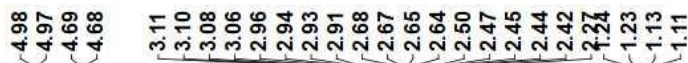

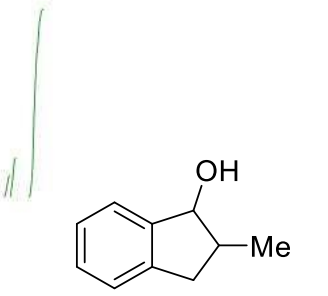

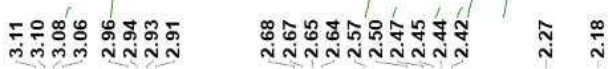

C

${ }^{1} \mathrm{H} \mathrm{NMR}\left(\mathrm{CDCl}_{3}, 500 \mathrm{MHz}\right)$

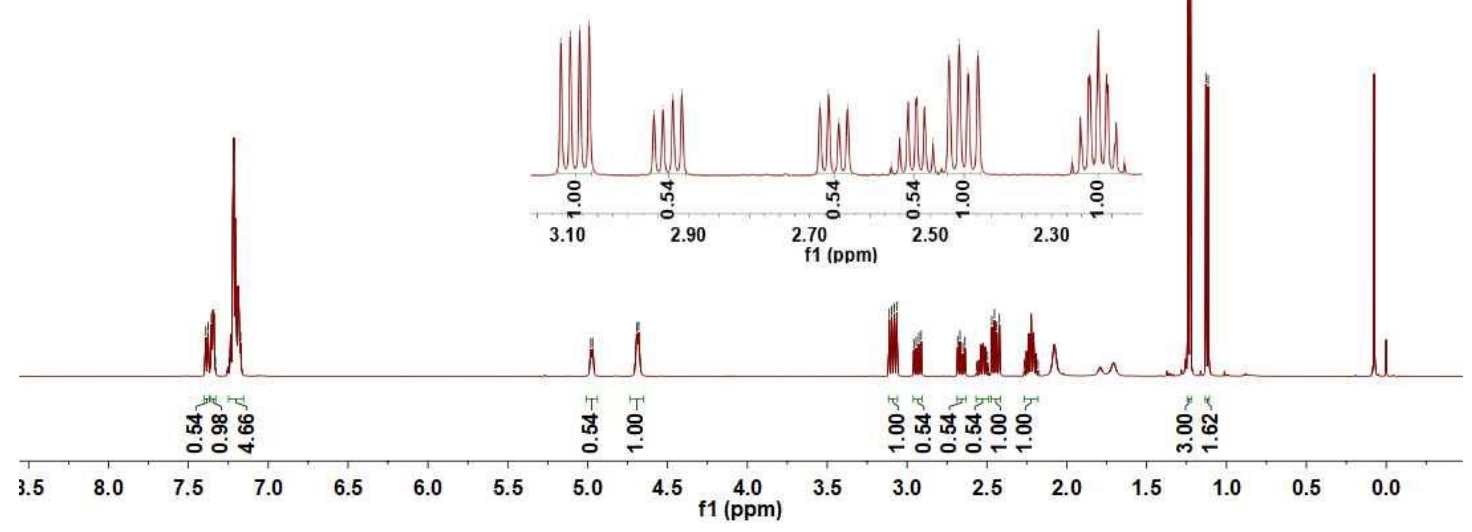

ํํำำ

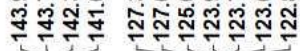

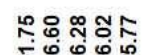

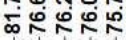

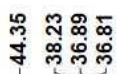

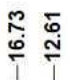

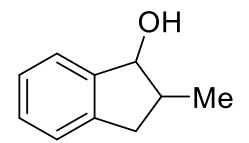

C

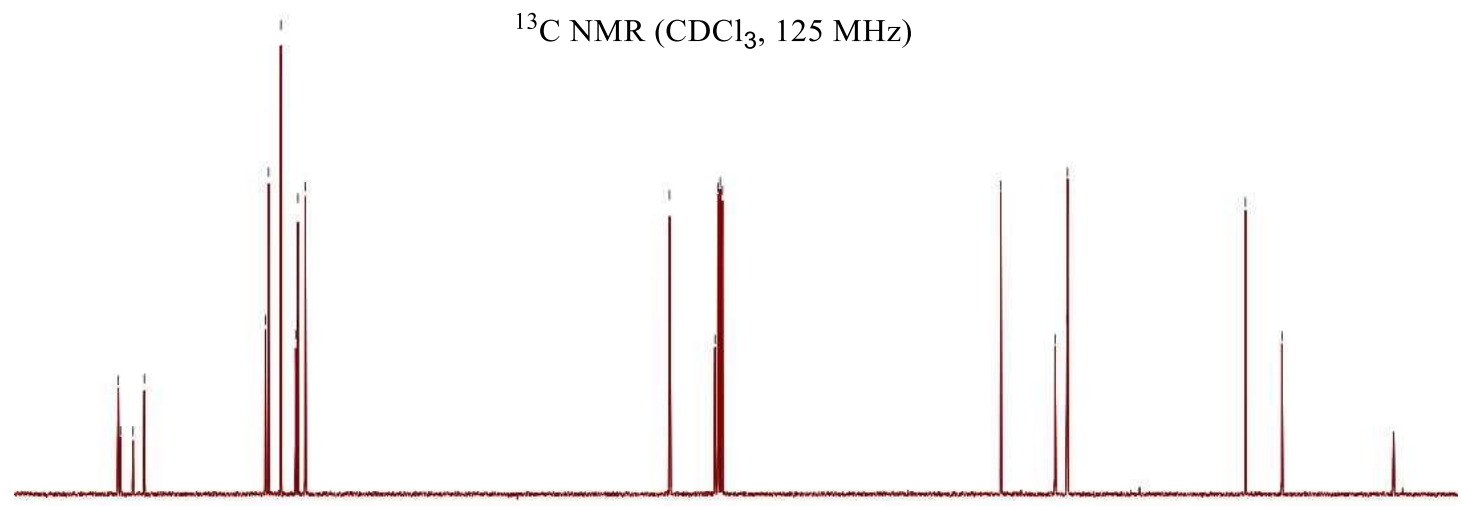

$\begin{array}{lllll}150 & 140 & 130 & 120 & 110\end{array}$

f1 (ppm)

60

$50 \quad 40$

30

$2010 \quad 0$ 


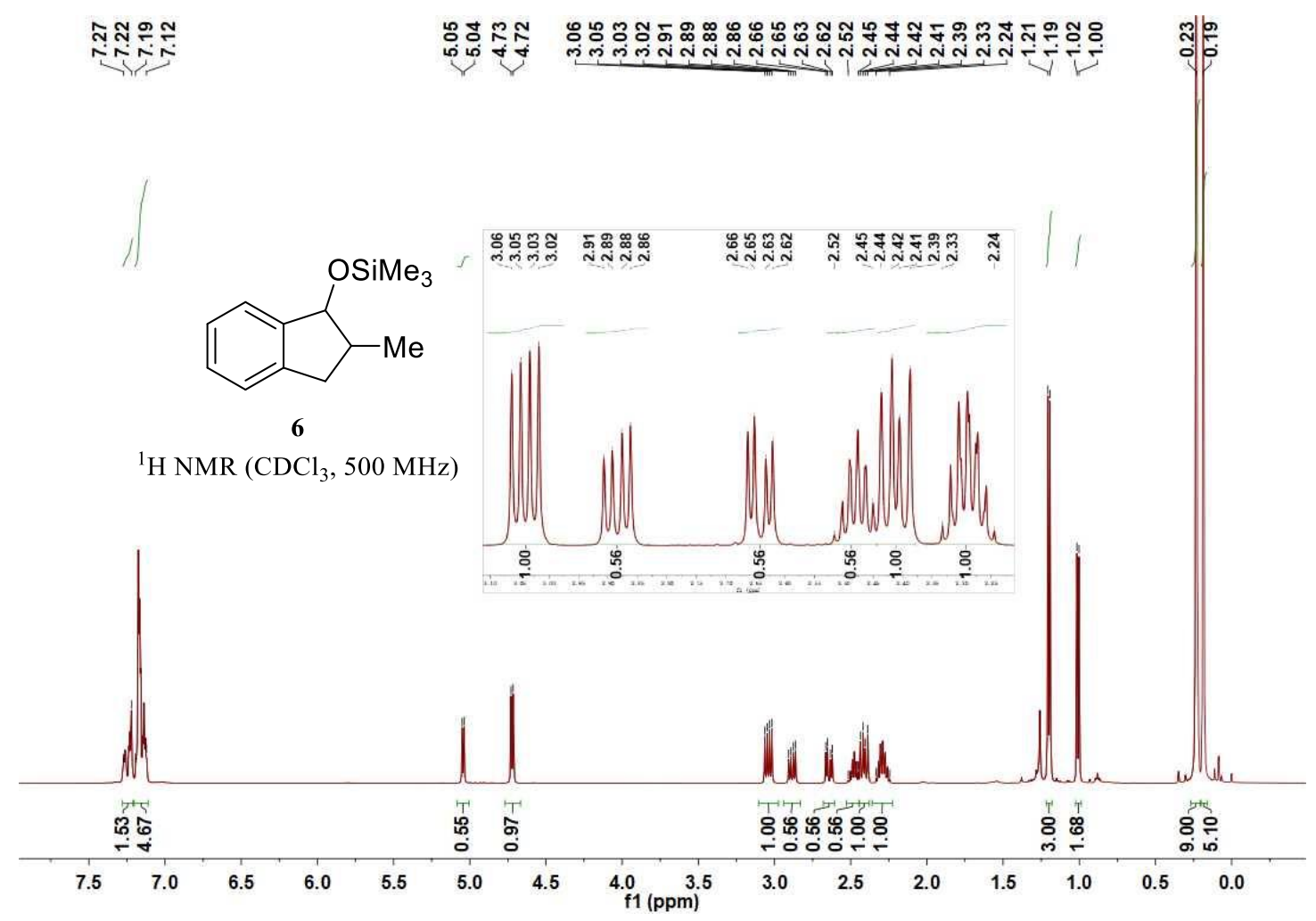

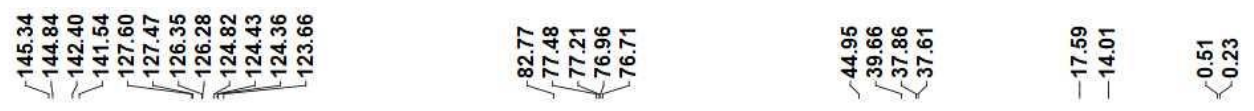

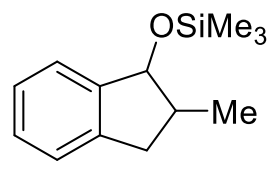

6

${ }^{13} \mathrm{C} \mathrm{NMR}\left(\mathrm{CDCl}_{3}, 125 \mathrm{MHz}\right)$

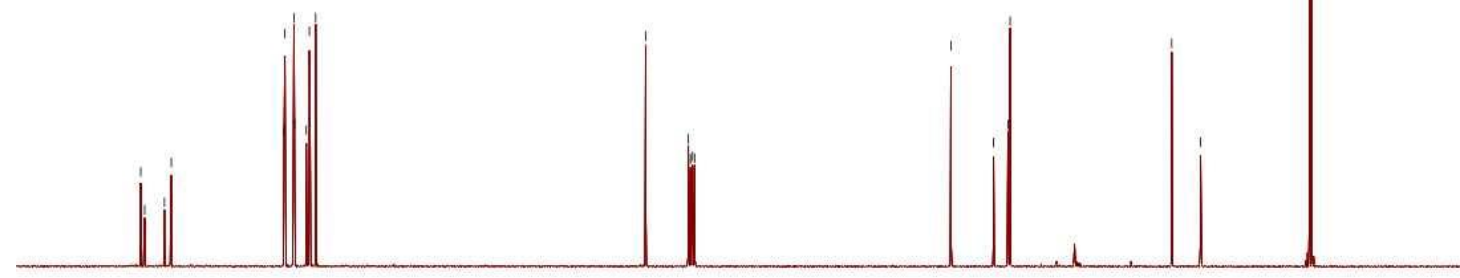

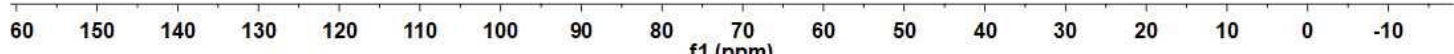



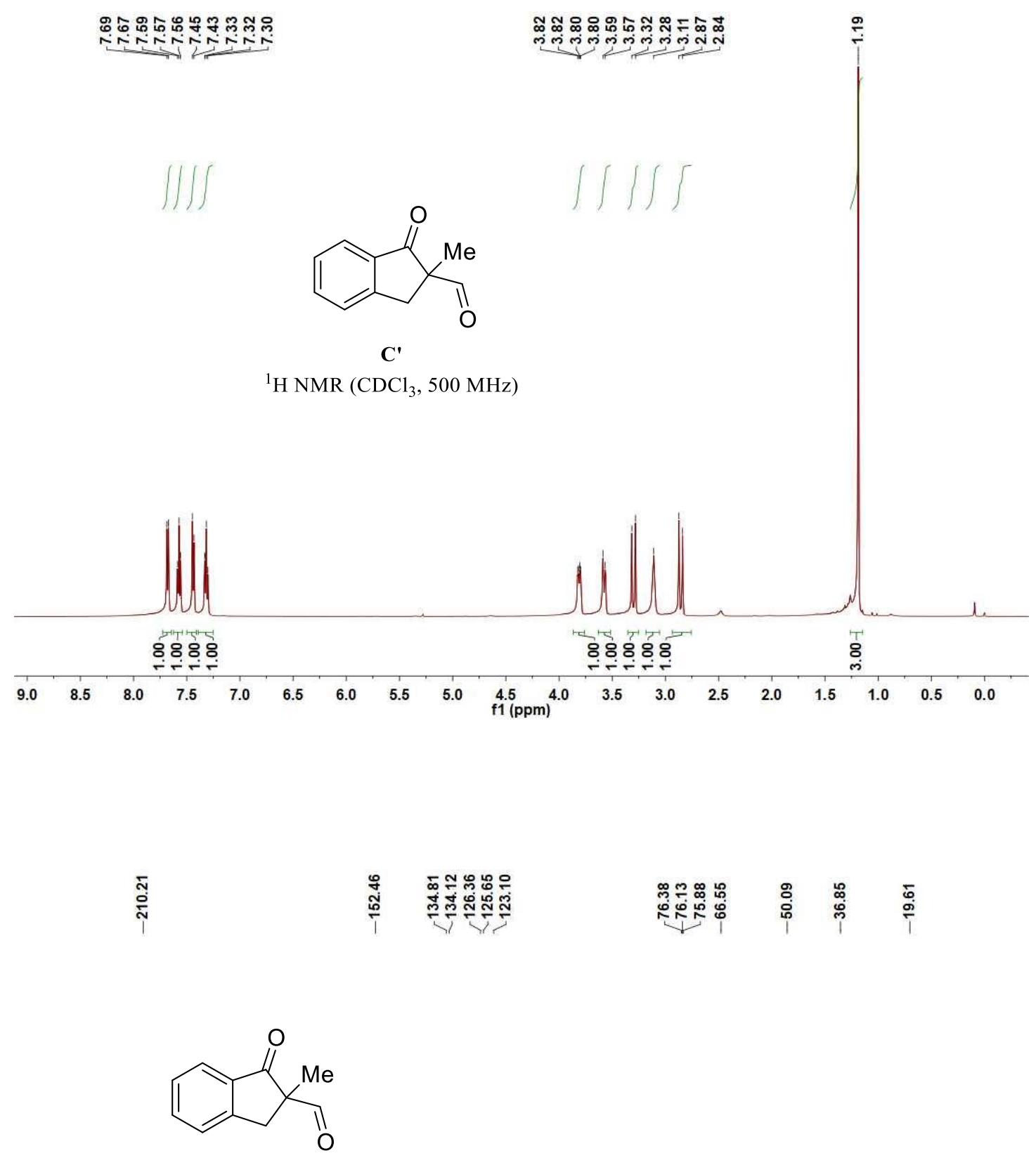

$C^{\prime}$

${ }^{13} \mathrm{C}$ NMR $\left(\mathrm{CDCl}_{3}, 125 \mathrm{MHz}\right)$

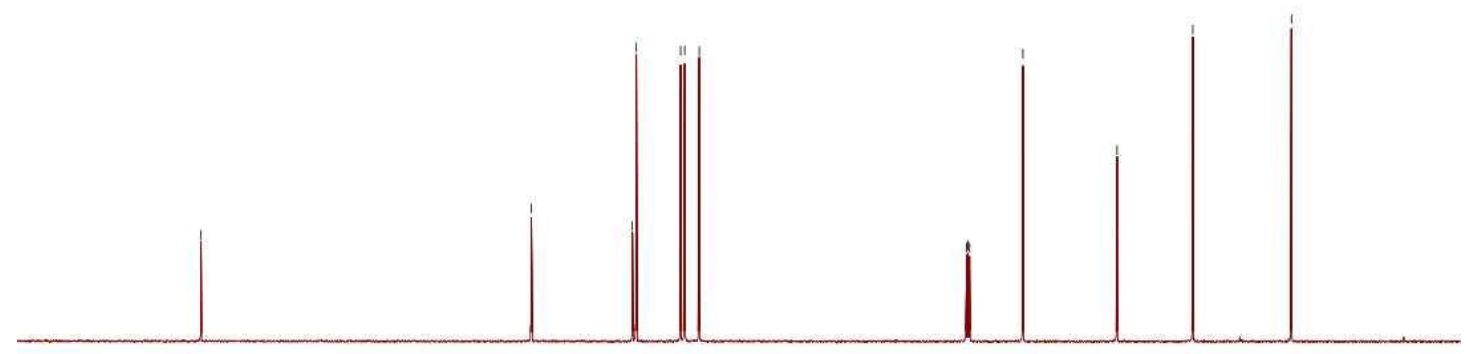

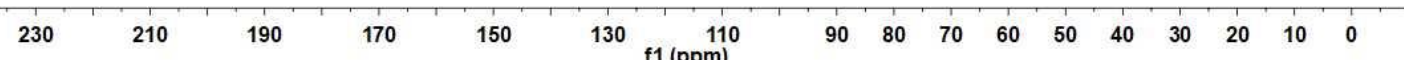




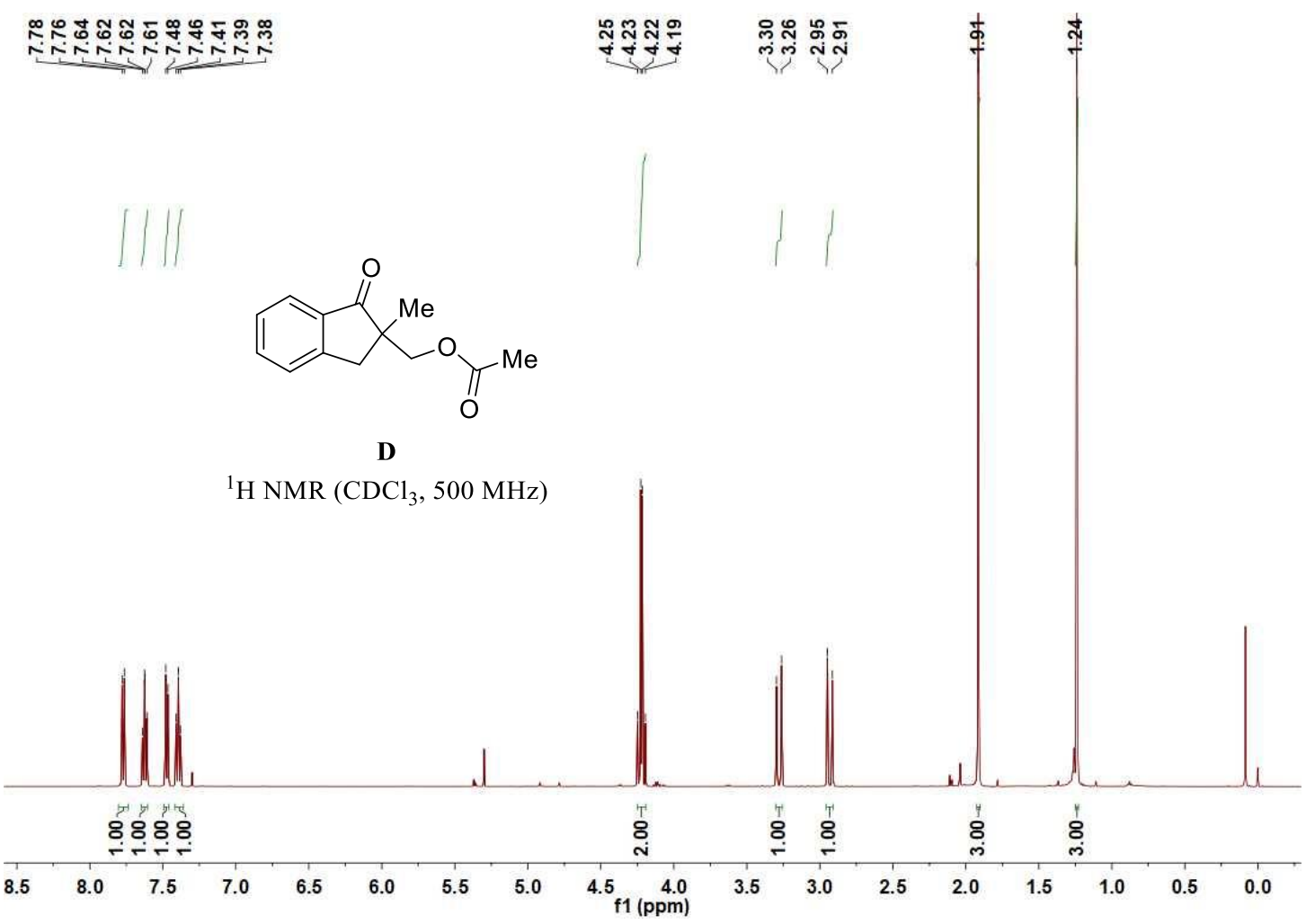

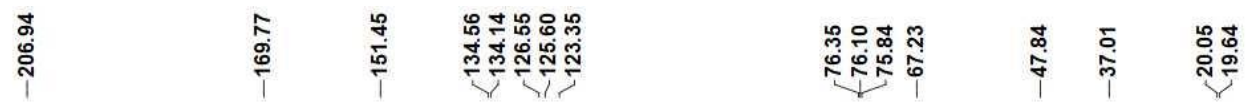

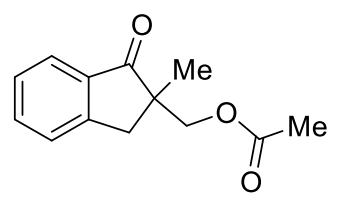

D

${ }^{13} \mathrm{C} \mathrm{NMR}\left(\mathrm{CDCl}_{3}, 125 \mathrm{MHz}\right)$

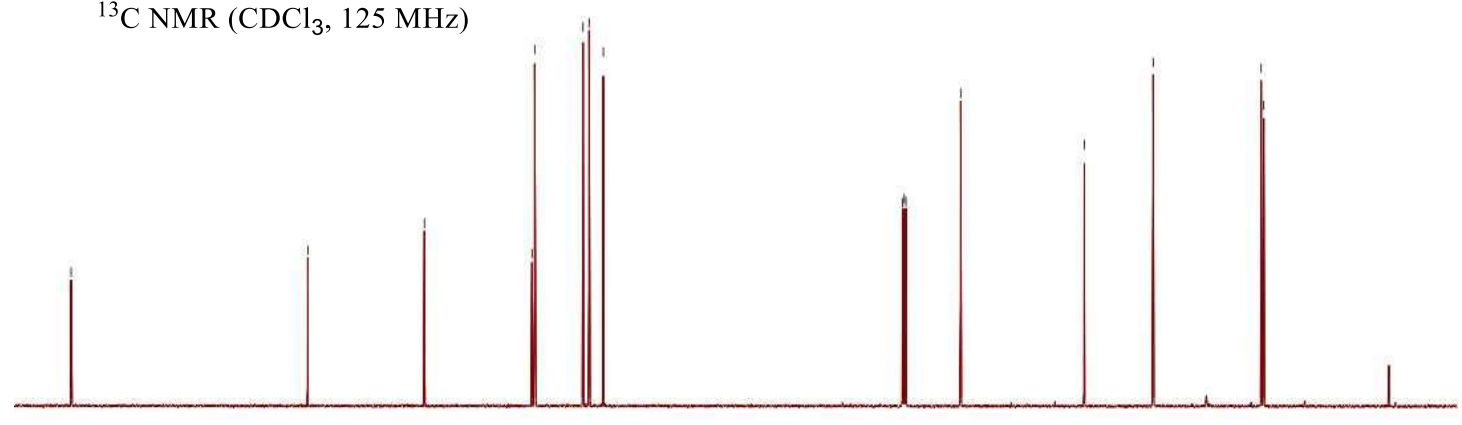

$\begin{array}{lllllllllllllllllllllllllllll}210 & 200 & 190 & 180 & 170 & 160 & 150 & 140 & 130 & 120 & 110 & 100 & 90 & 80 & 70 & 60 & 50 & 40 & 30 & 20 & 10 & 0 & -1\end{array}$ 

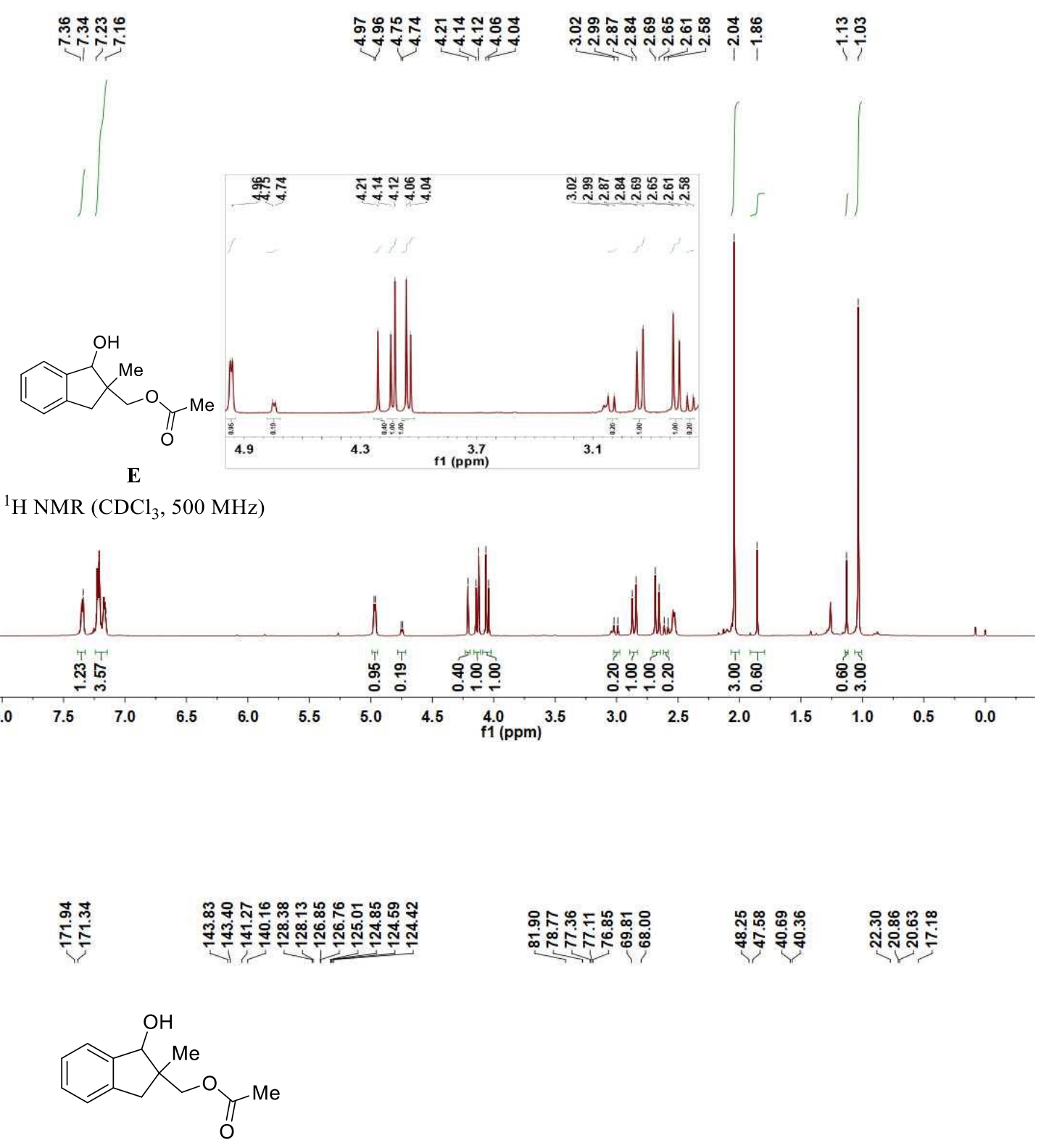

E

${ }^{13} \mathrm{C}$ NMR $\left(\mathrm{CDCl}_{3}, 125 \mathrm{MHz}\right)$

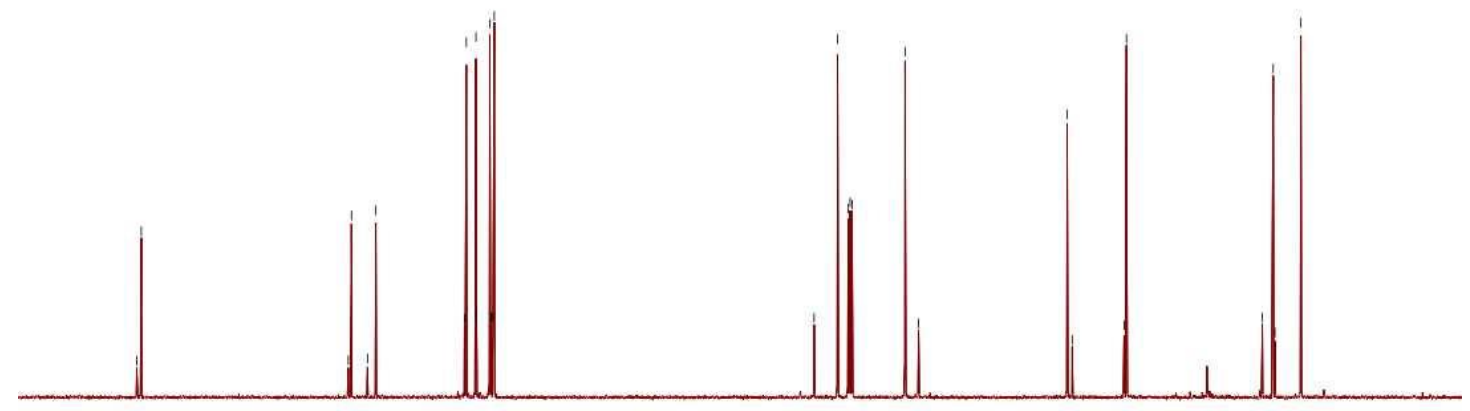

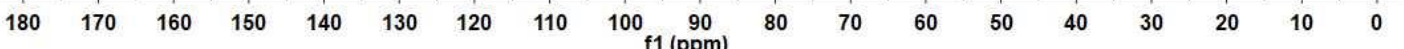



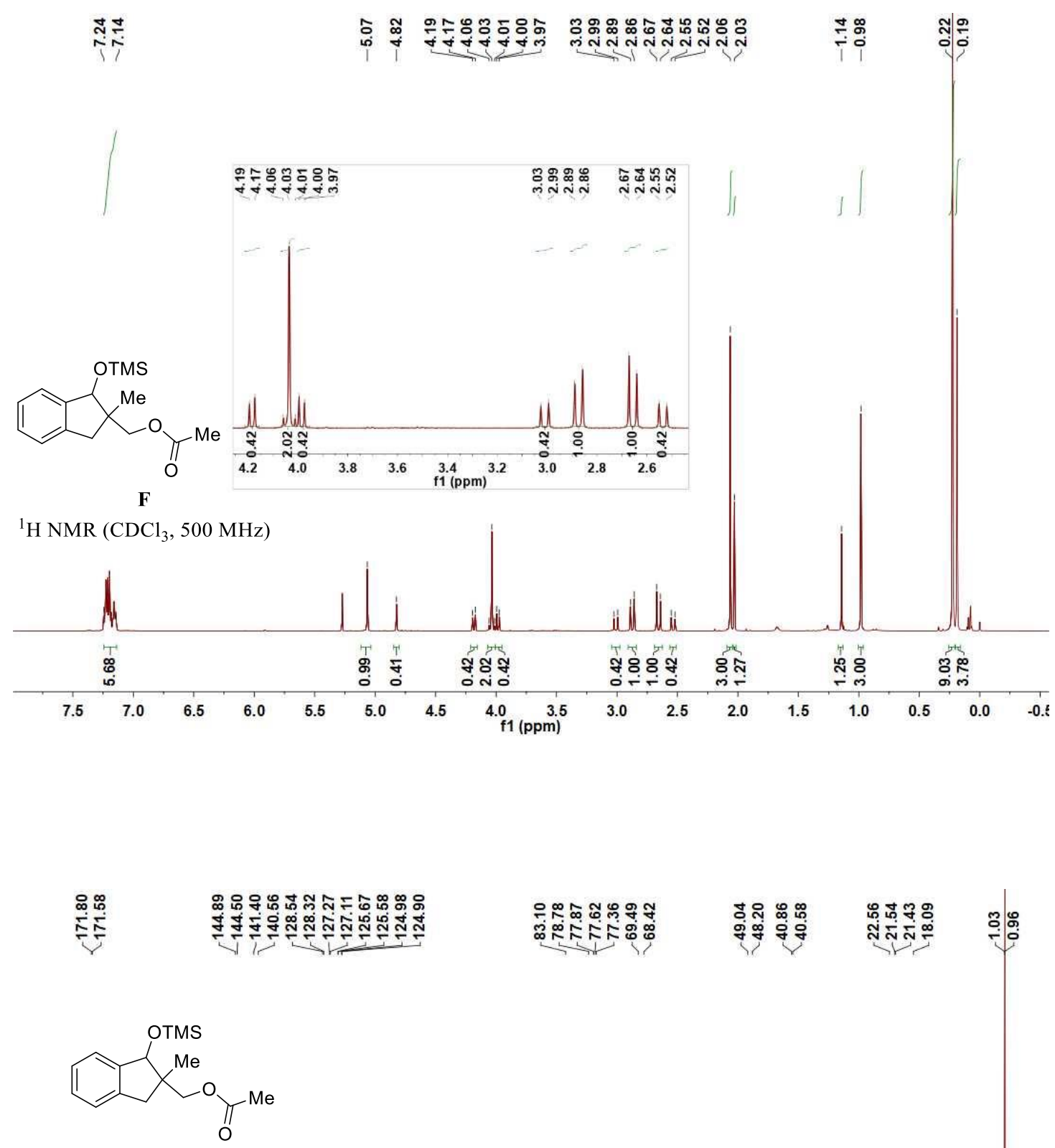

F

${ }^{13} \mathrm{C} \mathrm{NMR}\left(\mathrm{CDCl}_{3}, 125 \mathrm{MHz}\right)$

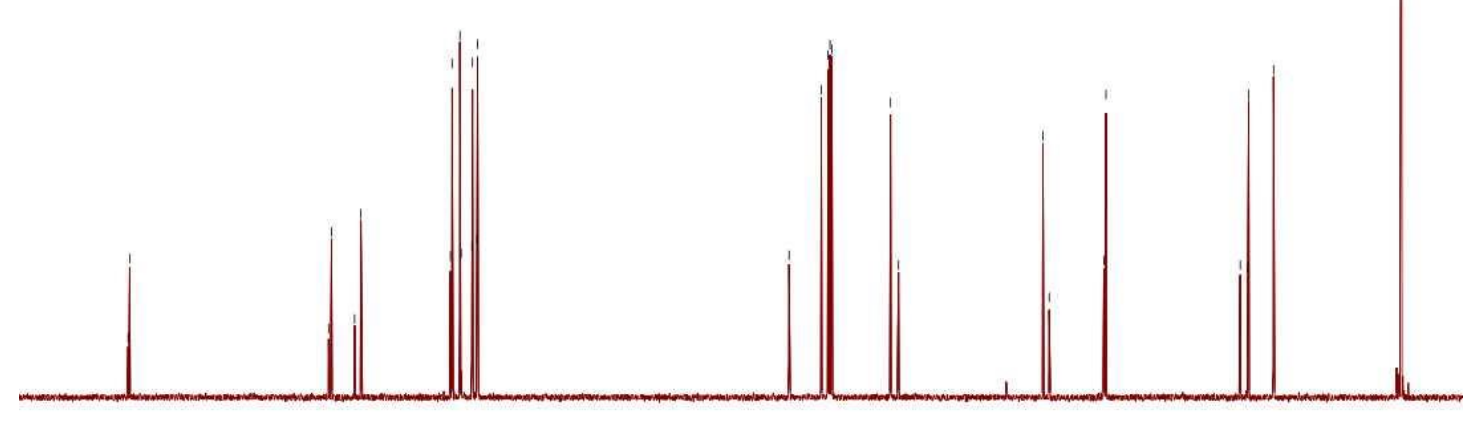

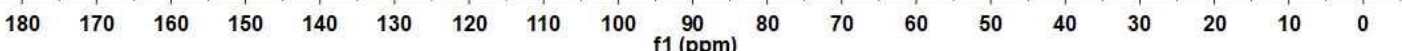




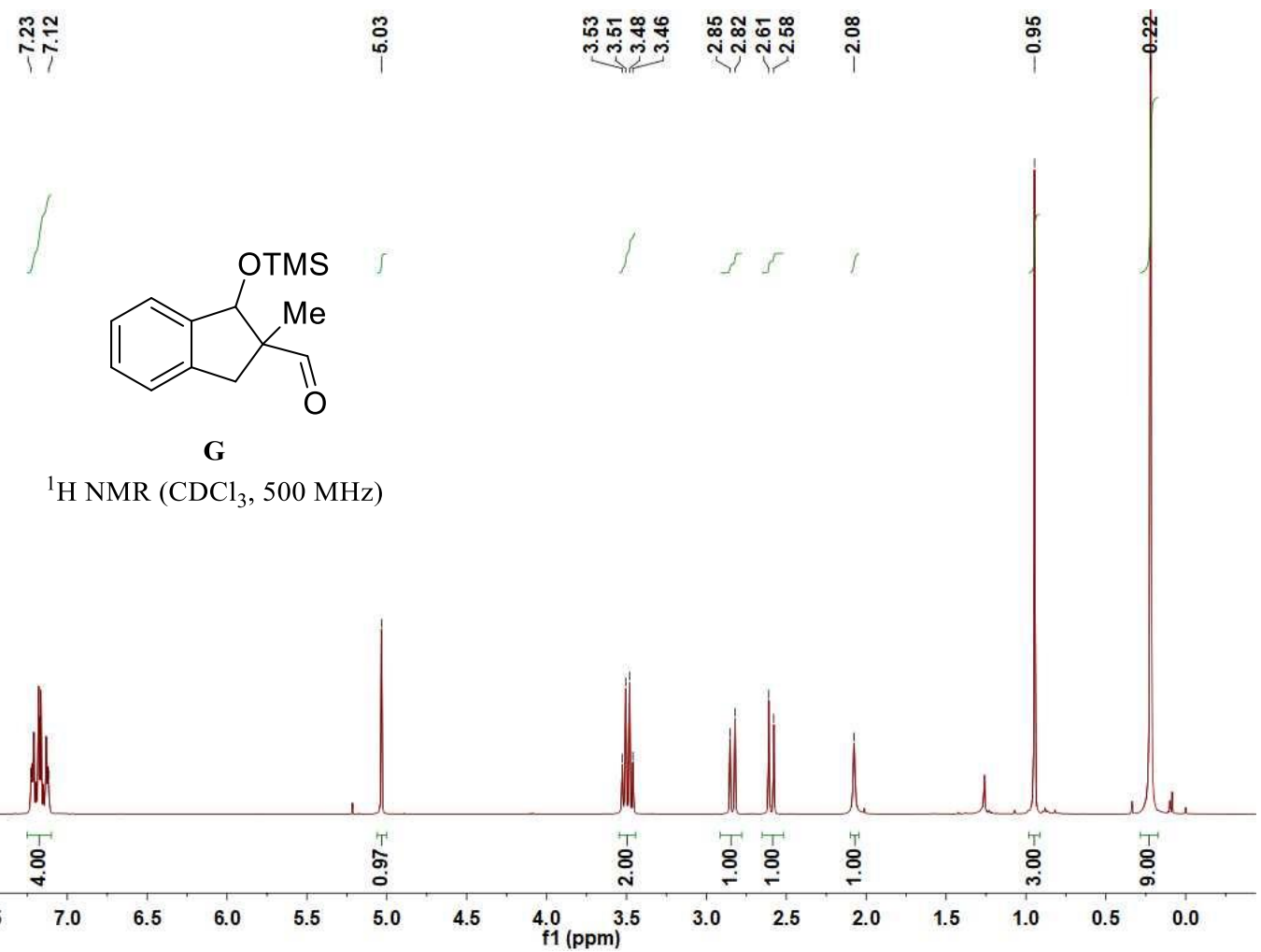

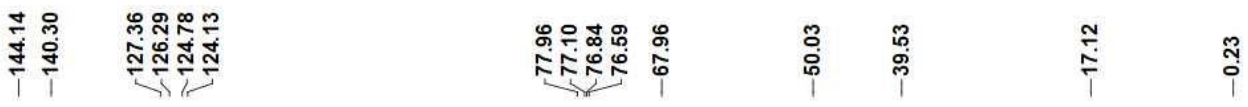

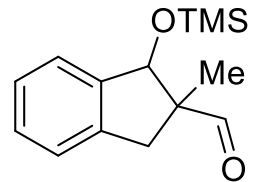

G

${ }^{13} \mathrm{C} \mathrm{NMR}\left(\mathrm{CDCl}_{3}, 125 \mathrm{MHz}\right)$
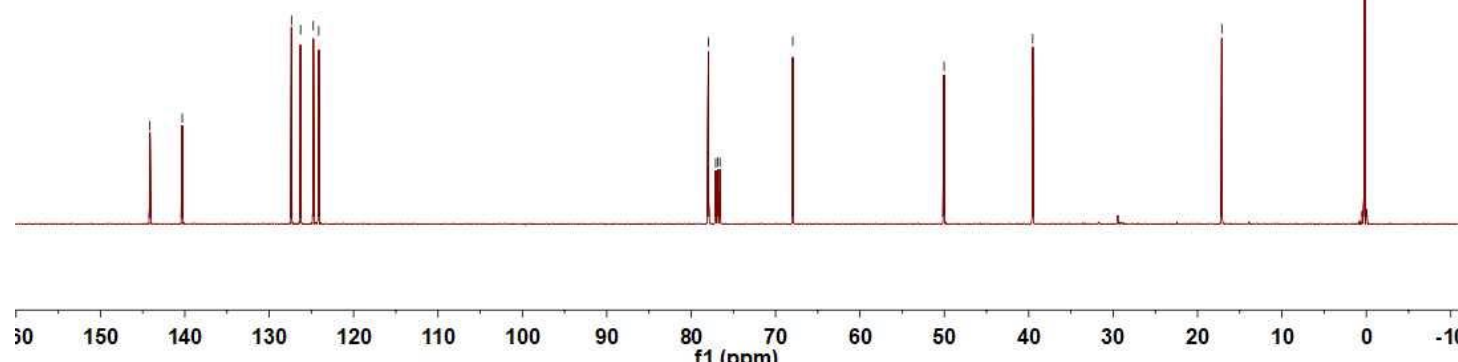

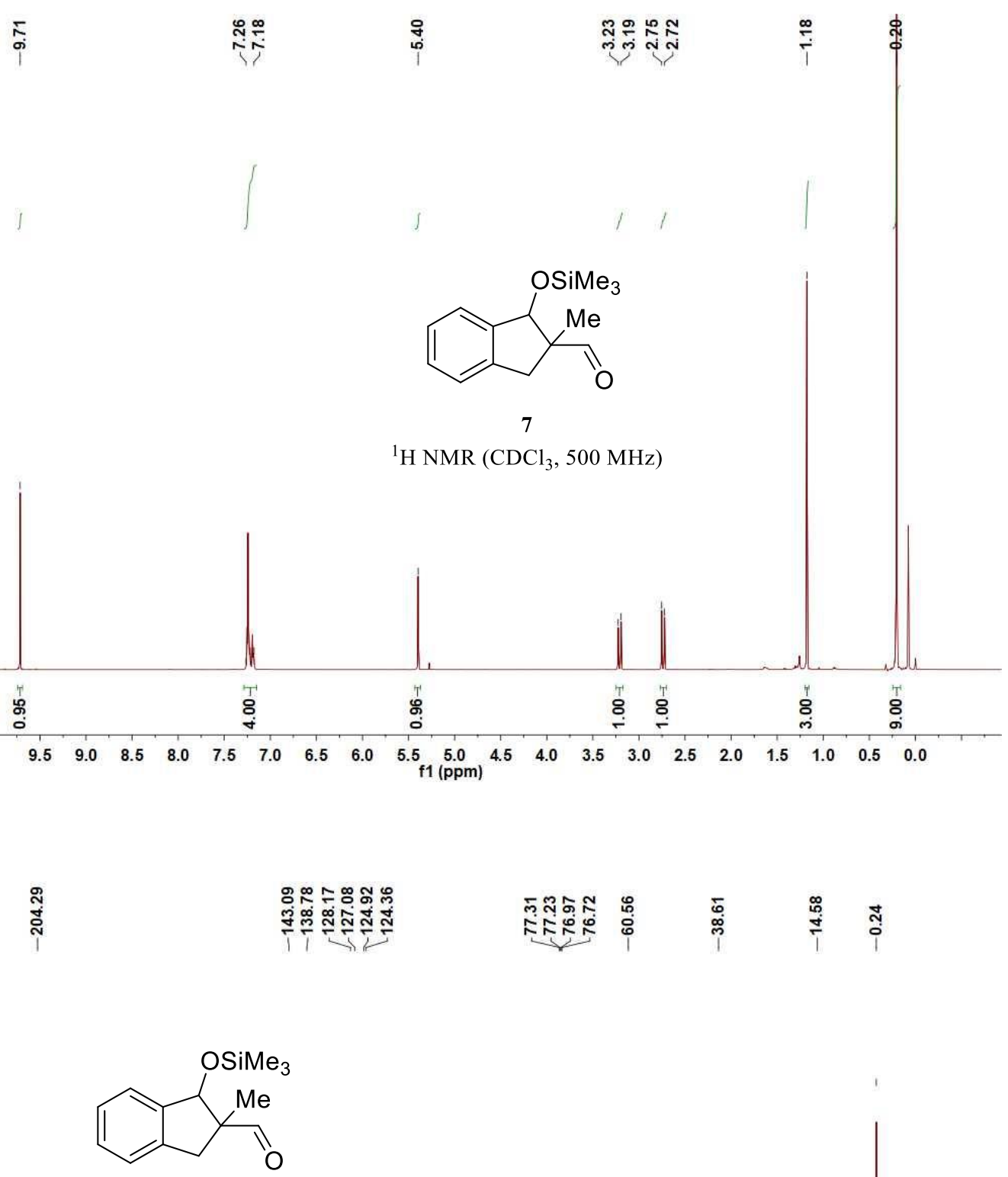

7

${ }^{13} \mathrm{C} \mathrm{NMR}\left(\mathrm{CDCl}_{3}, 125 \mathrm{MHz}\right)$

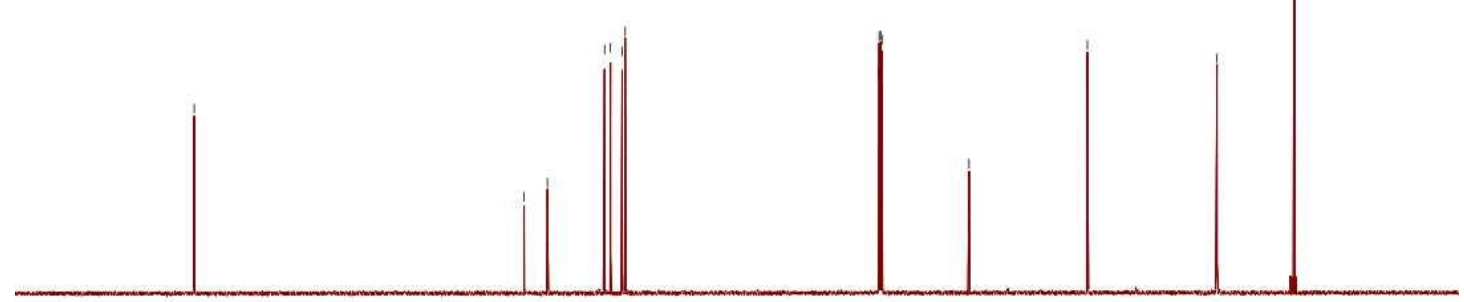

$\begin{array}{lllllllllllllllllll} & 230 & 210 & 190 & 170 & 150 & 130 & \underset{\mathrm{f} 1(\mathrm{ppm})}{110} & 90 & 80 & 70 & 60 & 50 & 40 & 30 & 20 & 10 & 0 & -20\end{array}$ 


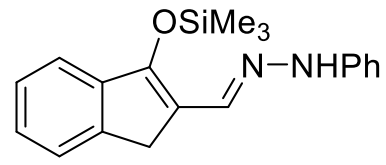

8

${ }^{1} \mathrm{H} \mathrm{NMR}\left(\mathrm{CDCl}_{3}, 500 \mathrm{MHz}\right)$

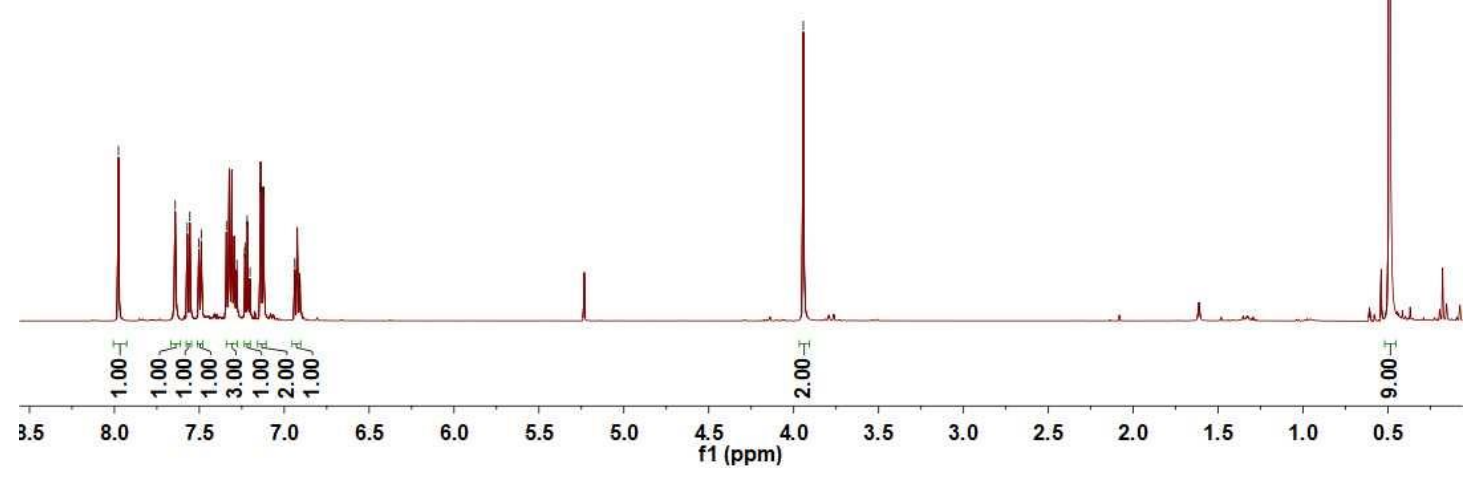

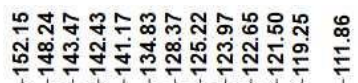

年里

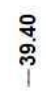

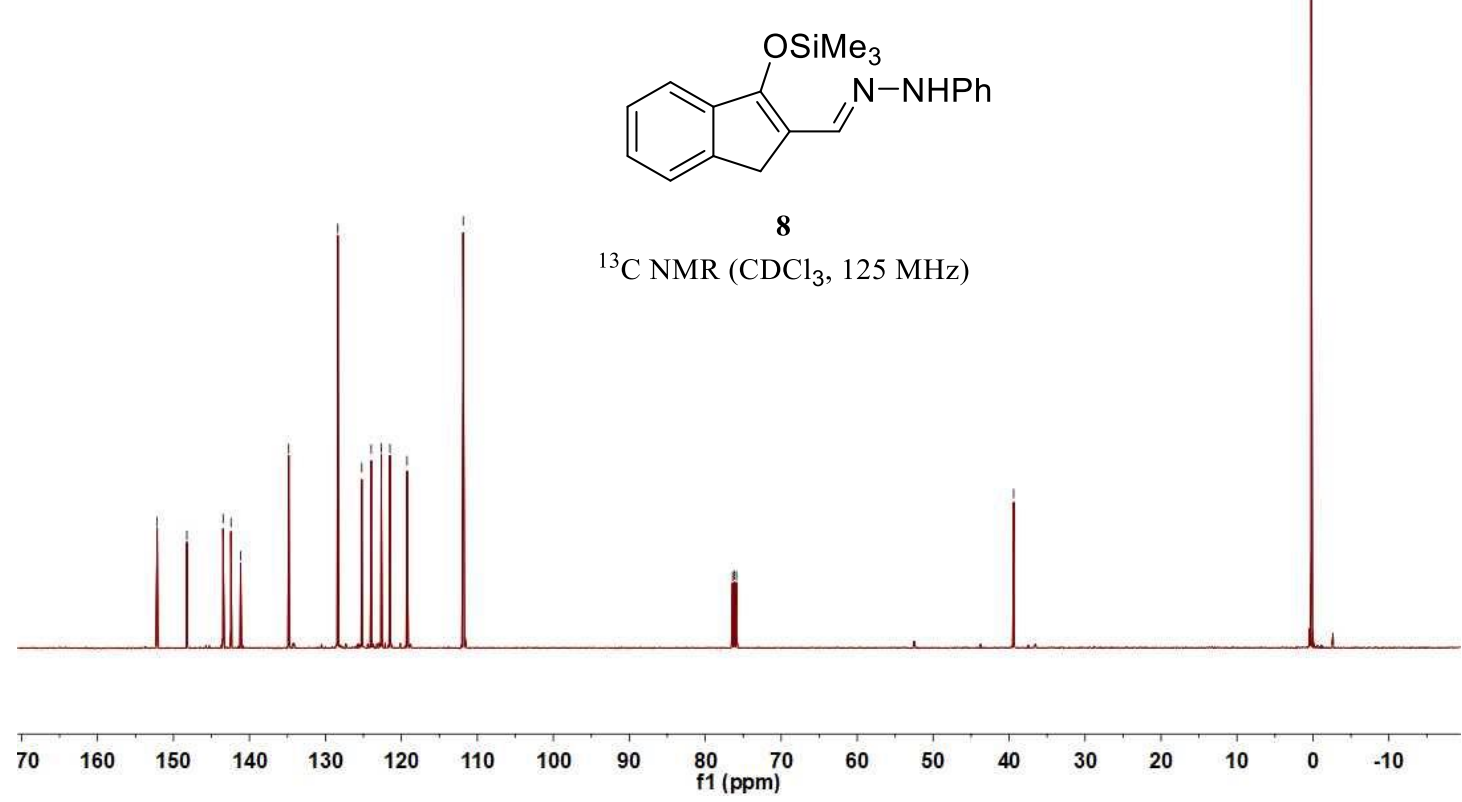

8

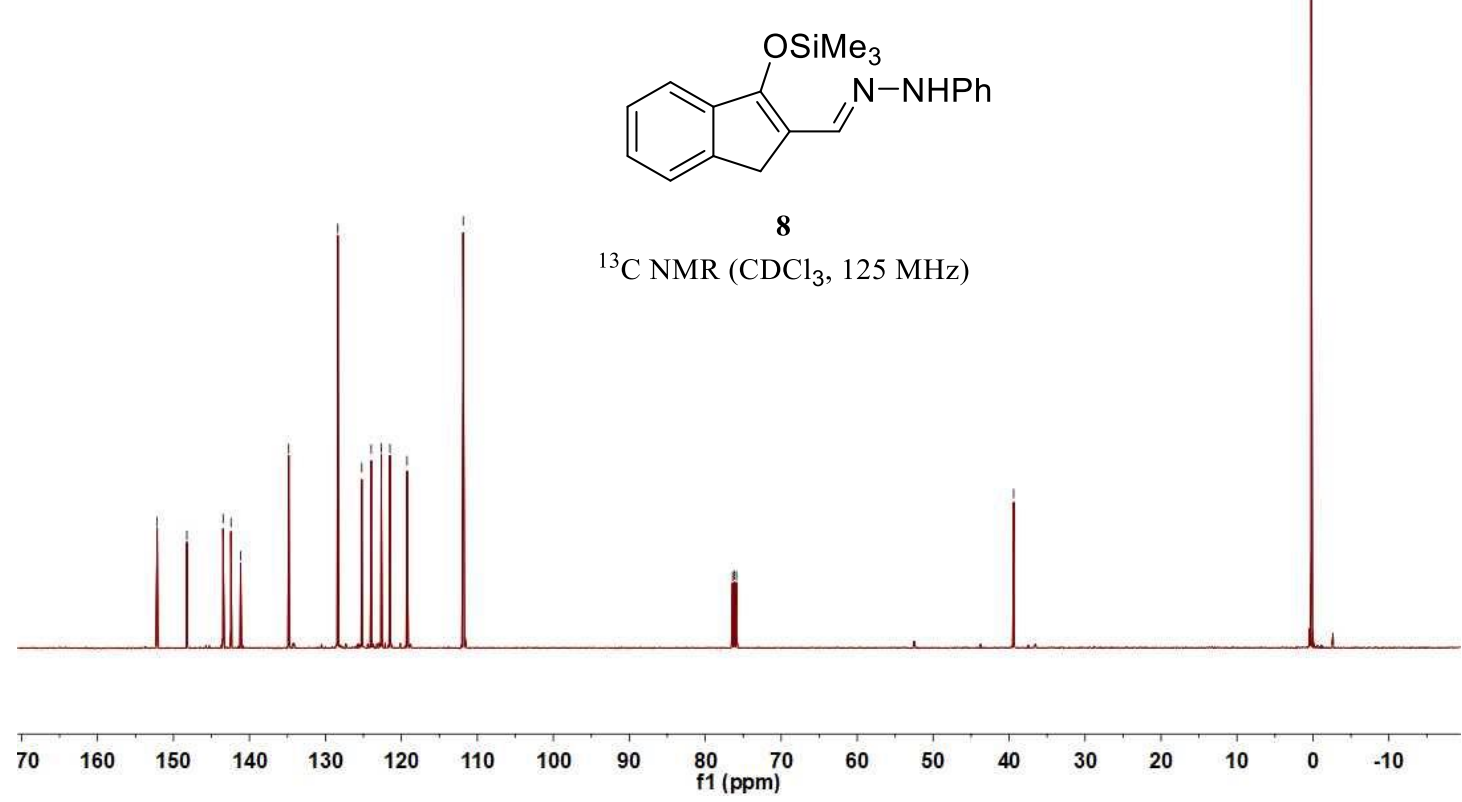



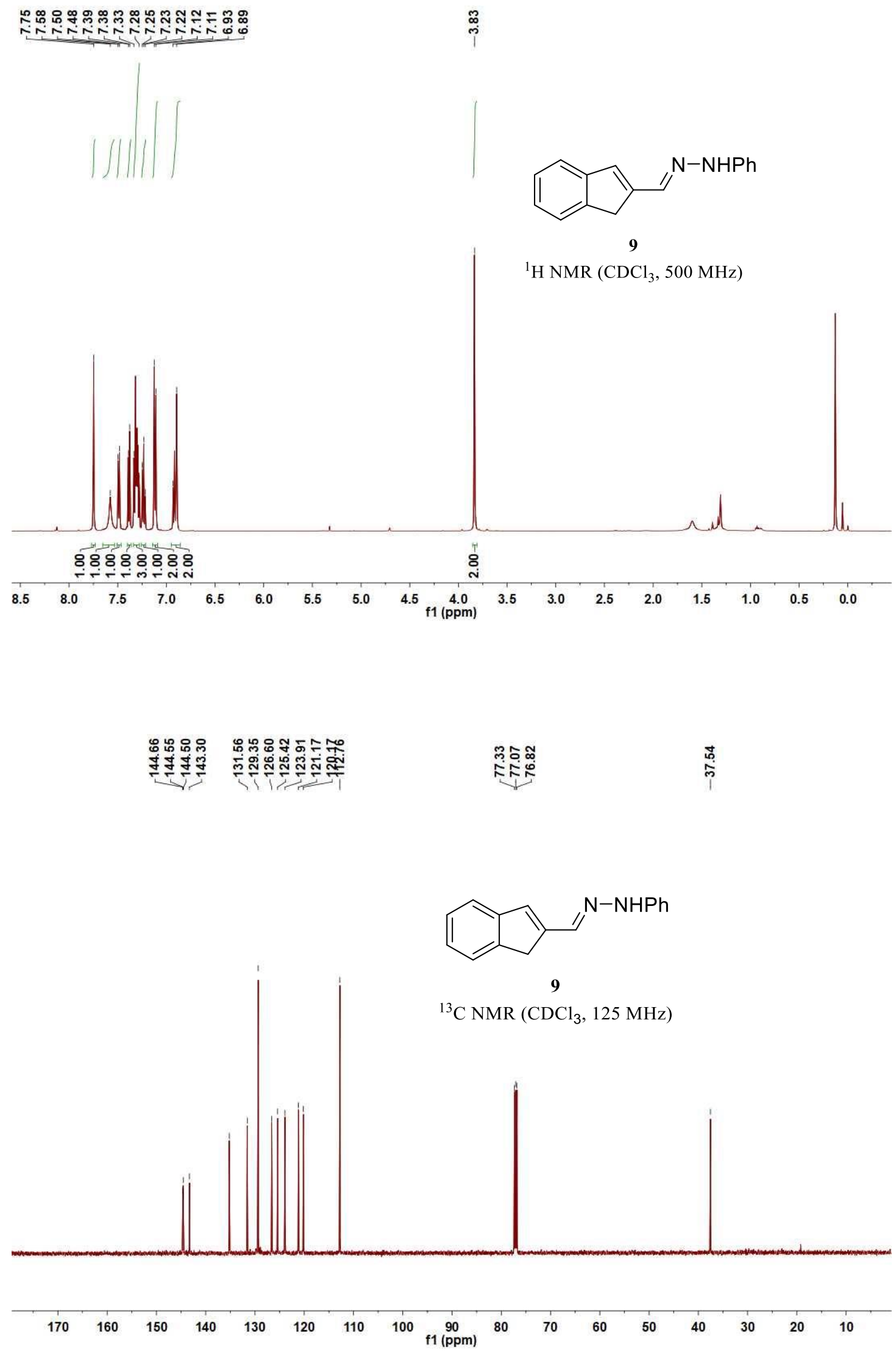


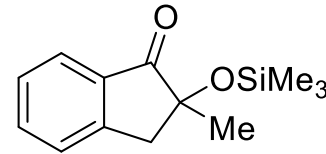

11

${ }^{1} \mathrm{H}$ NMR $\left(\mathrm{CDCl}_{3}, 500 \mathrm{MHz}\right)$

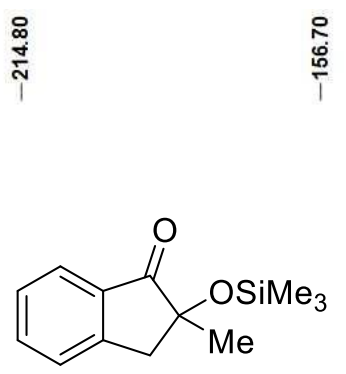

11

${ }^{13} \mathrm{C} \mathrm{NMR}\left(\mathrm{CDCl}_{3}, 125 \mathrm{MHz}\right)$

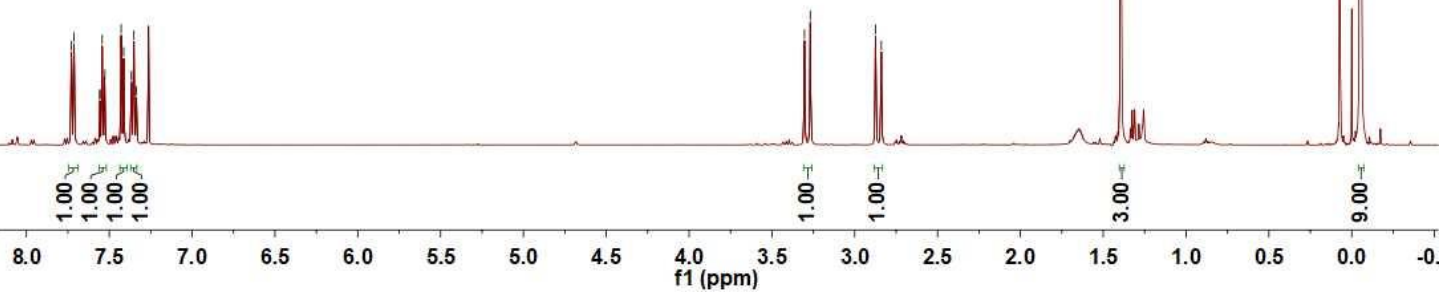

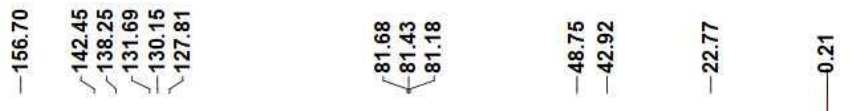

50 $230 \quad 210$ $190 \quad 170 \quad 150$ $130 \begin{gathered}110 \\ \mathrm{f} 1(\mathrm{ppm})\end{gathered}$ $\begin{array}{lllllllllll}90 & 80 & 70 & 60 & 50 & 40 & 30 & 20 & 10 & 0 & -10\end{array}$ 

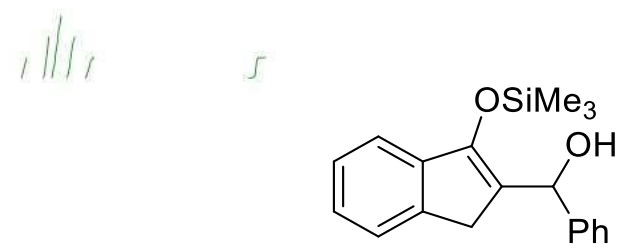

10

${ }^{1} \mathrm{H} \mathrm{NMR}\left(\mathrm{CDCl}_{3}, 500 \mathrm{MHz}\right)$
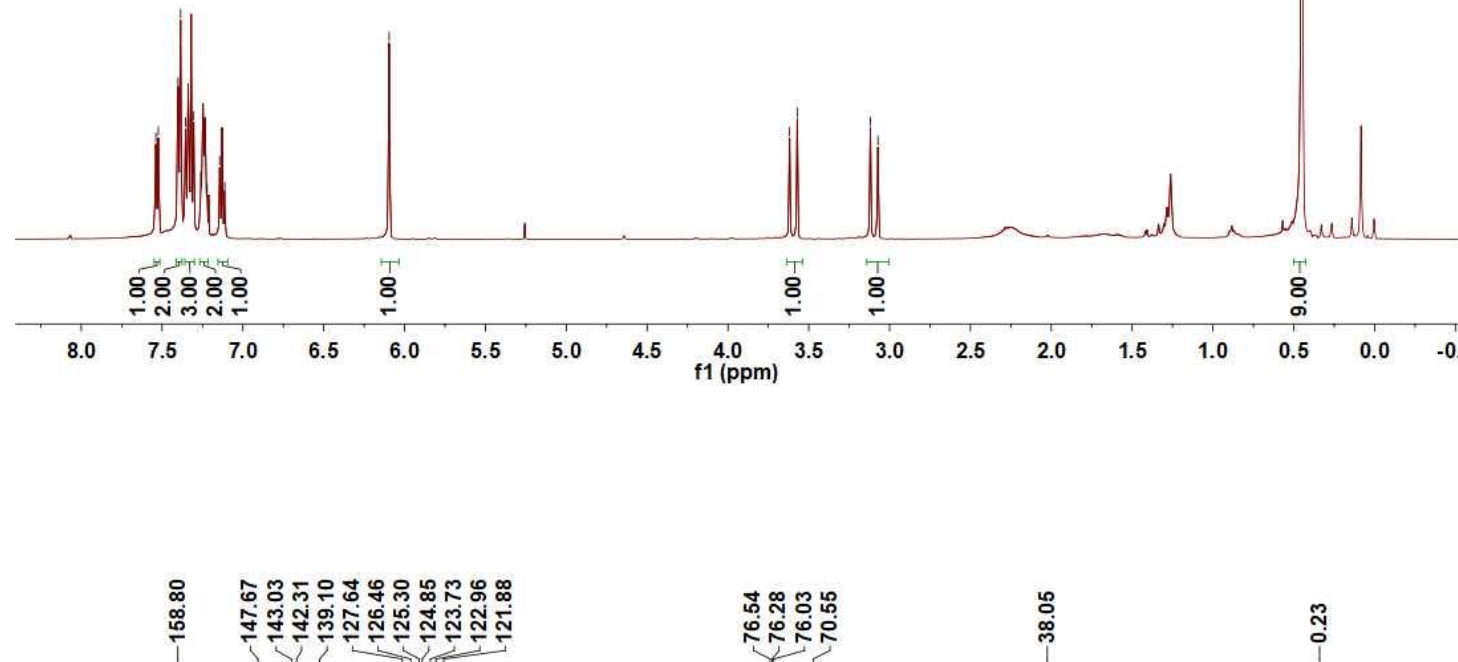

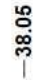

ָุi

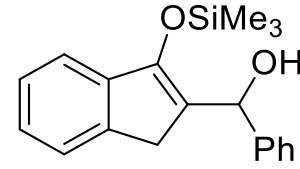

10

${ }^{13} \mathrm{C} \mathrm{NMR}\left(\mathrm{CDCl}_{3}, 125 \mathrm{MHz}\right)$

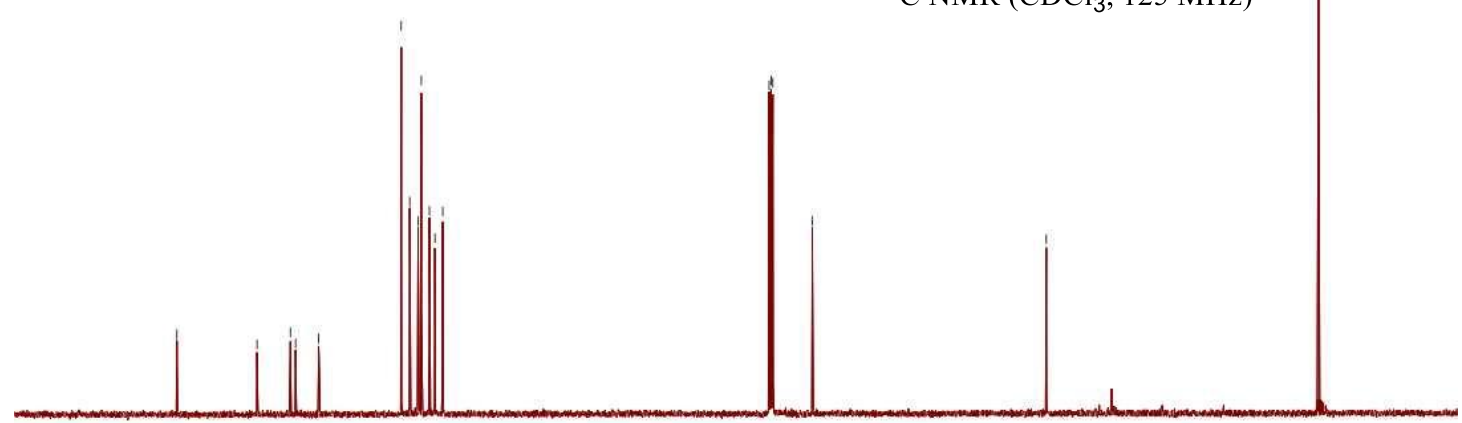

$\begin{array}{lllllllllllllllllll}80 & 170 & 160 & 150 & 140 & 130 & 120 & 110 & 100 & 90 \underset{\mathrm{f} 1(\mathrm{ppm})}{80} & 70 & 60 & 50 & 40 & 30 & 20 & 10 & 0 & -10\end{array}$ 